\title{
Immunometabolic effects of vitamin D unravelled by an integrative systems biology analysis
}

Citation for published version (APA):

Muñoz García, A. (2020). Immunometabolic effects of vitamin D unravelled by an integrative systems biology analysis: A collaborative bioinformatics and laboratory approach. [Doctoral Thesis, Maastricht University, University of Birmingham]. Maastricht University. https://doi.org/10.26481/dis.20200825ag

Document status and date:

Published: 01/01/2020

DOI:

10.26481/dis.20200825ag

Document Version:

Publisher's PDF, also known as Version of record

\section{Please check the document version of this publication:}

- A submitted manuscript is the version of the article upon submission and before peer-review. There can be important differences between the submitted version and the official published version of record.

People interested in the research are advised to contact the author for the final version of the publication, or visit the DOI to the publisher's website.

- The final author version and the galley proof are versions of the publication after peer review.

- The final published version features the final layout of the paper including the volume, issue and page numbers.

Link to publication

\footnotetext{
General rights rights.

- You may freely distribute the URL identifying the publication in the public portal. please follow below link for the End User Agreement:

www.umlib.nl/taverne-license

Take down policy

If you believe that this document breaches copyright please contact us at:

repository@maastrichtuniversity.nl

providing details and we will investigate your claim.
}

Copyright and moral rights for the publications made accessible in the public portal are retained by the authors and/or other copyright owners and it is a condition of accessing publications that users recognise and abide by the legal requirements associated with these

- Users may download and print one copy of any publication from the public portal for the purpose of private study or research.

- You may not further distribute the material or use it for any profit-making activity or commercial gain

If the publication is distributed under the terms of Article $25 \mathrm{fa}$ of the Dutch Copyright Act, indicated by the "Taverne" license above, 


\section{Immunometabolic effects of vitamin D unravelled by an integrative systems biology analysis}

\section{Citation for published version (APA):}

Muñoz García, A. (2020). Immunometabolic effects of vitamin D unravelled by an integrative systems biology analysis: A collaborative bioinformatics and laboratory approach. Maastricht University. https://doi.org/10.26481/dis.20200825ag

Document status and date:

Published: 01/01/2020

DOI:

10.26481/dis.20200825ag

Document Version:

Publisher's PDF, also known as Version of record

\section{Please check the document version of this publication:}

- A submitted manuscript is the version of the article upon submission and before peer-review. There can be important differences between the submitted version and the official published version of record.

People interested in the research are advised to contact the author for the final version of the publication, or visit the DOI to the publisher's website.

- The final author version and the galley proof are versions of the publication after peer review.

- The final published version features the final layout of the paper including the volume, issue and page numbers.

Link to publication

\footnotetext{
General rights rights.

- You may freely distribute the URL identifying the publication in the public portal. please follow below link for the End User Agreement:

www.umlib.nl/taverne-license

Take down policy

If you believe that this document breaches copyright please contact us at:

repository@maastrichtuniversity.nl

providing details and we will investigate your claim.
}

Copyright and moral rights for the publications made accessible in the public portal are retained by the authors and/or other copyright owners and it is a condition of accessing publications that users recognise and abide by the legal requirements associated with these

- Users may download and print one copy of any publication from the public portal for the purpose of private study or research.

- You may not further distribute the material or use it for any profit-making activity or commercial gain

If the publication is distributed under the terms of Article 25fa of the Dutch Copyright Act, indicated by the "Taverne" license above, 


\section{$\cos _{0}^{0} .00$ \\ IMMUNOMETABOLIC EFFECTS OF VITAMIN D UNRAVELLED BY AN INTEGRATIVE SYSTEMS BIOLOGY ANALYSIS \\ $\therefore 0^{\circ}$}

A collaborative bioinformatics and laboratory approach

AMADEO MUÑOZ GARCÍA

0
0000
0000

- $\quad 0000.000$ 



\title{
D. Maastricht \\ University
}

BIRMINGHAM

\section{Immunometabolic effects of vitamin D unravelled by an integrative systems biology analysis.}

\author{
A collaborative bioinformatics and laboratory approach
}

to obtain the degree of Doctor of Philosophy at the University of Birmingham and the degree of Doctor at Maastricht University, on the authority of the Rector Magnificus Prof.dr. Rianne M. Letschert in accordance with the decision of the Board of Deans on Tuesday $25^{\text {th }}$ of August 2020 at 13.00 hours in Maastricht. 


\section{Supervisors:}

Prof. Dr. C.T.A. Evelo

Prof. Dr. M. Hewison

\section{Co-supervisor:}

Dr. S.L.M. Coort

\section{Assessment Committee:}

Prof. Dr. I. Arts (chair Maastricht University)

Dr. K. Wouters

Dr. M. van Driel

Prof. Dr. G. Lavery (chair University of Birmingham)

Dr. R. Hardy

Dr. C. Hilkens 
A mis padres 



\section{Table of contents}

List of figures $\quad$ xi

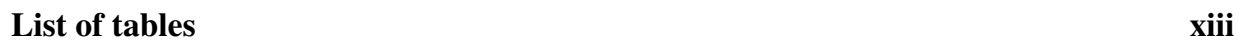

1 Introduction 1

1.1 History of vitamin $\mathrm{D} \ldots \ldots \ldots \ldots 2$

1.1.1 Discovery of Vitamin D . . . . . . . . . . . . . . 2

1.1.2 Vitamin D deficiency . . . . . . . . . . . . . 3

1.2 Metabolism of vitamin $\mathrm{D}$ and tissue-specific actions . . . . . . . . . 5

1.3 Immune system . . . . . . . . . . . . . . . . . . 8

1.3.1 Myeloid cells . . . . . . . . . . . . . . . . . . 8

1.3.2 Lymphoid cells . . . . . . . . . . . . . . . . . . 13

1.4 Role of vitamin D in different immune cell types . . . . . . . . . . . . . 15

1.4.1 Myeloid cells and vitamin D . . . . . . . . . . . . 16

1.4.2 Lymphoid cells and vitamin D . . . . . . . . . . . . . . . . . . . . . . . 17

1.5 Functional genomics analysis . . . . . . . . . . . . . 18

1.5.1 Biological databases . . . . . . . . . . . . . . . . . . 18

1.5.2 Transcriptomic data processing .............. 20

1.5 .3 Pathway analysis . . . . . . . . . . . . . 21

1.5.4 Integrative network analysis . . . . . . . . . . . . 23

1.6 Objectives. A return journey: from dry to wet lab . . . . . . . . . . 25

2 A bioinformatics workflow to decipher transcriptomic data from vitamin D $\begin{array}{ll}\text { studies } & 27\end{array}$

2.1 Abstract . . . . . . . . . . . . . . . . . . . 28

2.2 Introduction . . . . . . . . . . . . . . . . . 29

2.2 .1 Transcriptomic data . . . . . . . . . . . . . . . . . . 29

2.2.2 Bioinformatics tools . . . . . . . . . . . . . . 29 
2.2.3 Data analysis workflow . . . . . . . . . . . . . 30

2.3 Materials and methods . . . . . . . . . . . . . . . . 32

2.3.1 Retrieving gene expression data from public repositories . . . . . 32

2.3.2 Sharing large-scale expression studies . . . . . . . . . . . 32

2.3.3 Selection vitamin D-related transcriptomic studies in immune cells . 32

2.3.4 Processing raw gene expression data: ArrayAnalysis . . . . . . . . 33

2.3.5 ArrayAnalysis modules . . . . . . . . . . . . . 33

2.3.6 Analysis of selected vitamin D-related transcriptomics datasets . . . 34

2.3.7 Creation of vitamin D related processes . . . . . . . . . . . . . 34

2.3.8 Pathway analysis: visualization and statistics . . . . . . . . 35

2.3.9 Reporter metabolite analysis . . . . . . . . . . . . . . 37

2.3.10 Extension of biological pathway diagrams from WikiPathways in

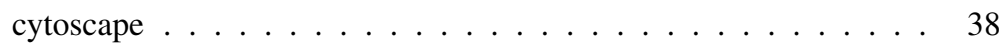

2.4 Results and discussion $\ldots \ldots \ldots \ldots$. . . . . . . . . . . . . . 39

2.4 .1 Workflow . . . . . . . . . . . . . . . 39

2.4.2 Pathway analysis using public datasets: vitamin D and gene expression modulation in myeloid cells . . . . . . . . . . . . . . . . 39

2.4.3 Pathway editing and data visualization: from references to dynamic pathways . . . . . . . . . . . . . . . . 39

2.4.4 Integrating multiple datasets in PathVisio . . . . . . . . . . . . . 42

2.4.5 Reporter metabolite analysis . . . . . . . . . . . . . . . 42

2.4.6 Extension of the PI3K/Akt/mTOR-vitamin D3 signaling pathway with approved drugs, validated microRNAs and curated pathways . 44

2.5 Conclusion . . . . . . . . . . . . . . . . . . . . . . 46

3 Transcriptomic analysis of vitamin D responses in uterine and peripheral NK $\begin{array}{ll}\text { cells } & 47\end{array}$

3.1 Abstract . . . . . . . . . . . . . . . . . . . . 48

3.2 Introduction . . . . . . . . . . . . . . . . . . 48

3.3 Methods . . . . . . . . . . . . . . . . . . . . . 49

3.3.1 Ethical approval . . . . . . . . . . . . . . . . 49

3.3.2 Isolation of NK cells from peripheral blood and decidua . . . . . . 49

3.3 .3 Cell culture . . . . . . . . . . . . . . . . . . 50

3.3.4 Flow cytometric analysis of CD69 and VDR . . . . . . . . . 50

3.3.5 Confocal imaging of VDR in CD56+ pNKs . . . . . . . . . . . 50

3.3.6 Quantitative real-time PCR (qRT-PCR) analysis . . . . . . . . . . . 51

3.3.7 RNA sequence analysis (RNA-seq) . . . . . . . . . . . . 51 
3.3.8 Bioinformatics and pathway analysis . . . . . . . . . . . 52

3.4 Results . . . . . . . . . . . . . . . . . . . . . 53

3.4.1 Comparison of gene expression in cytokine (CK)-stimulated $\mathrm{pNK}$ and uNK cells . . . . . . . . . . . . . . . . . . 53

3.4.2 VDR expression in uNK is upregulated following cytokine stimulation 57

3.4.3 Effects of $1,25(\mathrm{OH})_{2} \mathrm{D} 3$ on gene expression in CK-stimulated $\mathrm{pNK}$ and uNK cells . . . . . . . . . . . . . . . . . . . 61

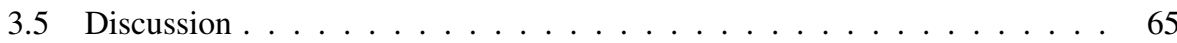

4 Pathway analysis of transcriptomic data shows immunometabolic effects of vitamin D 69

4.1 Abstract ........................ 70

4.2 Introduction . . . . . . . . . . . . . . . . 70

4.3 Materials and methods . . . . . . . . . . . . . . . . 72

4.3.1 Workflow overview . . . . . . . . . . . . . 72

4.3.2 Transcriptomic datasets . . . . . . . . . . . . . . 74

4.3.3 Pre-processing raw data and statistical data analysis in ArrayAnalysis 74

4.3.4 Pathway statistics and analysis in PathVisio . . . . . . . . . . . 75

4.3.5 GO analysis with ClueGO in Cytoscape . . . . . . . . . . . . 77

4.4 Results . . . . . . . . . . . . . . . . . . . . 77

4.4.1 Differentially expressed genes in $1,25(\mathrm{OH})_{2} \mathrm{D} 3$-treated THP1 cells, dendritic cells and monocytes . . . . . . . . . . . 77

4.4.2 Pathway analysis reveals altered biological processes based on molecular changes in $1,25(\mathrm{OH})_{2} \mathrm{D} 3$-treated immune cells . . . . . . . 79

4.4.3 GO analysis shows metabolic, immunological and apoptotic processes altered by $1,25(\mathrm{OH})_{2} \mathrm{D} 3$ in THP-1 cells, dendritic cells and monocytes .................... 79

4.4.4 Visualization of $1,25(\mathrm{OH})_{2} \mathrm{D} 3$-induced changes in gene expression in THP-1 cells, DCs and monocytes on altered metabolic pathways 80

4.4.5 $1,25(\mathrm{OH})_{2} \mathrm{D} 3$ and the electron transport chain . . . . . . . . 84

4.4.6 $1,25(\mathrm{OH})_{2} \mathrm{D} 3$ and oxidative phosphorylation $\ldots \ldots . . . . .88$

4.4.7 $1,25(\mathrm{OH})_{2} \mathrm{D} 3$ and the TCA cycle and glycolysis $\ldots \ldots . \ldots 84$

4.4.8 $1,25(\mathrm{OH})_{2} \mathrm{D} 3$ and cell proliferation $\ldots \ldots \ldots$. . . . . . . 89

$4.4 .9 \quad 1,25(\mathrm{OH})_{2} \mathrm{D} 3$ and apoptosis $\ldots \ldots \ldots \ldots$

4.5 Discussion . . . . . . . . . . . . . . . . . . . 95

4.6 Conclusions . . . . . . . . . . . . . . . . . . . . . . 98 
5 Tolerogenic effects of vitamin D on DCs involve induction of fatty acid synthesis 99

5.1 Abstract . . . . . . . . . . . . . . . . . . . . . . . 100

5.2 Introduction . . . . . . . . . . . . . . . . . . . . . 100

5.3 Materials \& Methods . . . . . . . . . . . . . . . . . . . . . . . 102

5.3.1 Isolation of primary human peripheral blood monocytes . . . . . . 102

5.3.2 In vitro generation of monocyte-derived DC . . . . . . . . . . . . . 102

5.3.3 Flow cytometry analysis of DC . . . . . . . . . . . . . 103

5.3.4 Microscope fluorescence imaging . . . . . . . . . . . . . . . 105

5.3.5 Quantitative Real-time PCR . . . . . . . . . . . . . . . 105

5.3.6 Measurement of oxygen consumption . . . . . . . . . . . . 105

5.3.7 Mass spectrometry analysis of ${ }^{13} \mathrm{C}_{6}$-glucose and ${ }^{13} \mathrm{C}_{5}$-glutamine metabolism . . . . . . . . . . . . . . . . 106

5.3.8 Statistical analysis . . . . . . . . . . . . . 106

5.4 Results . . . . . . . . . . . . . . . . . . . . . . . . . . 107

5.4.1 $1,25(\mathrm{OH})_{2} \mathrm{D} 3$ regulates DC phenotype in the presence and absence of immunogenic stimulus . . . . . . . . . . . . . . . . . . 107

5.4.2 1,25( $(\mathrm{OH})_{2} \mathrm{D} 3$-induced itolDC are characterised by increased oxidative phosphorylation . . . . . . . . . . . . . 107

5.4.3 1,25(OH $)_{2} \mathrm{D} 3$-induced itolDC show increased TCA cycle metabolism 113

5.4.4 $1,25(\mathrm{OH})_{2} \mathrm{D} 3$-induces fatty acid synthesis in itolDC $\ldots \ldots 115$

5.4.5 Inhibition of fatty acid synthesis suppresses itolDC induction by $1,25(\mathrm{OH})_{2} \mathrm{D} 3 \ldots \ldots \ldots \ldots \ldots \ldots \ldots$

5.5 Discussion . . . . . . . . . . . . . . . . . 115

6 General discussion $\quad 121$

6.1 General discussion . . . . . . . . . . . . . . . . . . . . . 121

6.2 Bioinformatic pipelines for functional omics data analysis . . . . . . . . . 121

6.2.1 Discovery of new molecular aspects of vitamin D by exploiting public gene expression datasets . . . . . . . . . . . . . 122

6.2.2 Understanding biological systems using integration of omics data . 123

6.2.3 Automatization and integration of open bioinformatic tools . . . . . 124

6.3 Vitamin D and immunometabolism . . . . . . . . . . . . . . . . . . . . 125

6.3.1 $1,25(\mathrm{OH})_{2} \mathrm{D} 3$ modulates immune cell function which is accompanied by a metabolic reprogramming . . . . . . . . . . . . 125

6.4 Therapeutic applications . . . . . . . . . . . . . . . . . . . 126

6.5 Vitamin D supplementation in personalized medicine . . . . . . . . . . . . 127 
6.6 Final words . . . . . . . . . . . . . . . . . . . . 128

$\begin{array}{lr}\text { Summary } & 129\end{array}$

$\begin{array}{ll}\text { Resumen } & 135\end{array}$

$\begin{array}{ll}\text { Valorization } & 141\end{array}$

$\begin{array}{ll}\text { Acknowledgements } & 145\end{array}$

$\begin{array}{ll}\text { References } & 151\end{array}$

$\begin{array}{lll}\text { Appendix A } & \text { List of Abbreviations } & 171\end{array}$

$\begin{array}{llr}\text { Appendix B Curriculum Vitae } & 179\end{array}$ 



\section{List of figures}

1.1 Major contributors who led to the discovery of vitamin D . . . . . . . . 3

1.2 The endocrinology of vitamin D. . . . . . . . . . . . 6

1.3 Schematic representation of hematopoietic stem cell (HSC) development. . 9

1.4 Autocrine and paracrine actions of vitamin D in innate immune cells and its effects on adaptive immune cells. . . . . . . . . . . . . . . 16

1.5 The application of bioinformatic tools for analysis of transcriptomic datasets. 19

1.6 A role for bioinformatics in the interpretation and design of experimental research. ......................... 26

2.1 Bioinformatics workflow for the analysis of transcriptomic data . . . . . . . 31

2.2 Visualization of gene expression changes in myeloid cells in the proposed model from Ferreira et al. 2015 [72] using PathVisio . . . . . . . . . . . 36

2.3 Extended PI3K/Akt/mTOR-vitamin D3 signalling pathway with drug-target, microRNA-target and gene-pathway interactions. . . . . . . . . . . . 45

3.1 Transcriptomic analysis of cytokine (CK) stimulated pNK and uNK cells. . $\quad 54$

3.2 Pathway analysis of vitamin D receptor (VDR) signalling-related genes in CK-stimulated uNK versus CK-stimulated pNK . . . . . . . . . . . . 55

3.3 Expression of VDR in paired peripheral blood and uterine natural killer cells. 58

3.4 Pathway analysis of transcriptomic responses to $1,25(\mathrm{OH})_{2} \mathrm{D} 3$-treated in THP-1 cells, dendritic cells, monocytes and B cells compared to effects in pNK and uNK . . . . . . . . . . . . . . . . . . . . . . . . 59

3.5 Pathway analysis of genes associated with non-genomic responses to $1,25(\mathrm{OH})_{2} \mathrm{D} 3$ in CK-stimulated uNK versus CK-stimulated pNK. . . . . . . . . . . . 62

3.6 Pathway analysis of HIF1A and PPARG regulation of glycolysis-related genes in CK-stimulated uNK versus CK-stimulated pNK . . . . . . . . . . 63

3.7 Pathway analysis of the Cori cycle in CK-stimulated uNK versus CKstimulated $\mathrm{pNK} \ldots \ldots \ldots \ldots \ldots$. . . . . . . . . . . . . . 64 
4.1 Overview of the analytical tools included in the workflow . . . . . . . . . 73

4.2 Heat map showing common altered pathways in $1,25(\mathrm{OH})_{2} \mathrm{D} 3$-treated in different immune cell models. . . . . . . . . . . . . . . . . . . . 78

4.3 Venn diagram showing numbers of common and cell-specific genes regulated by $1,25(\mathrm{OH})_{2} \mathrm{D} 3 \ldots \ldots \ldots \ldots$

4.4 ClueGO gene ontology analysis of common $1,25(\mathrm{OH})_{2} \mathrm{D} 3$-regulated bioenergetics genes ......................... . . 81

4.5 ClueGO gene ontology analysis of common $1,25(\mathrm{OH})_{2} \mathrm{D} 3$-regulated immune related processes genes . . . . . . . . . . . . . . . . 82

4.6 ClueGO gene ontology analysis of common $1,25(\mathrm{OH})_{2} \mathrm{D} 3$-regulated apoptosis related processes genes . . . . . . . . . . . . . . . . 83

4.7 Effect of $1,25(\mathrm{OH})_{2} \mathrm{D} 3$ on genes associated with the electron transport chain 85

4.8 Effect of $1,25(\mathrm{OH})_{2} \mathrm{D} 3$ on genes associated with the glycolysis . . . . . . 86

4.9 Effect of $1,25(\mathrm{OH})_{2} \mathrm{D} 3$ on genes associated with oxidative phosphorylation. 87

4.10 Effect of $1,25(\mathrm{OH})_{2} \mathrm{D} 3$ on genes associated with the TCA cycle. . . . . . . 88

4.11 Effect of $1,25(\mathrm{OH})_{2} \mathrm{D} 3$ on the expression of genes associated with cell proliferation in THP-1 cells . . . . . . . . . . . . . . . . . . . 90

4.12 Effect of $1,25(\mathrm{OH})_{2} \mathrm{D} 3$ on the expression of genes associated with DNA replication in THP-1 cells . . . . . . . . . . . . . . . . . . . . 91

4.13 Effect of $1,25(\mathrm{OH})_{2} \mathrm{D} 3$ on genes associated with apoptosis modulation by HSP70. WikiPathways representation of apoptosis modulation by HSP70. . 92

4.14 Effect of $1,25(\mathrm{OH})_{2} \mathrm{D} 3$ on genes associated with apoptosis. . . . . . . . . . 93

4.15 Heat map showing temporal effect of $1,25(\mathrm{OH})_{2} \mathrm{D} 3$ treatment in THP-1 cells. 94

5.1 Regulation of DC phenotype by $1,25(\mathrm{OH})_{2} \mathrm{D} 3 . \ldots \ldots$. . . . . . . 108

$5.21,25(\mathrm{OH})_{2} \mathrm{D} 3$-induced oxidative phosphorylation in itolDC. . . . . . . . . . 109

5.3 Tracer metabolite analysis of glucose metabolism in iDC and itolDC . . . . 110

5.4 Tracer metabolite analysis of glutamine metabolism in iDC and itolDC. . . 111

5.5 Tracer metabolite analysis of glucose metabolism to fatty acid precursors in iDC and itolDC . . . . . . . . . . . . . . . . . . . . . . 112

5.6 Inhibition of fatty acid synthesis suppresses effects of $1,25(\mathrm{OH})_{2} \mathrm{D} 3$ on CD14 and IL-10 in itolDC. . . . . . . . . . . . . . . . . . . . . . 114 


\section{List of tables}

1.1 Main immune cell models that will be mention/studied in higher detail in this thesis. ....................... 12

2.1 List of pathways significantly altered by vitamin D in different myeloid cells models. . . . . . . . . . . . . . . . . 4 41

2.2 Reporter metabolite analysis (RMA) results. . . . . . . . . . . . . . 43

3.1 Pathway analysis of differential gene expression in $\mathrm{CK}$ uNK versus CK pNK 56

3.2 Effect of $1,25(\mathrm{OH})_{2} \mathrm{D} 3$ on gene expression in $\mathrm{CK}$ pNK and uNK. . . . . . 60

4.1 Regulation of gene expression by $1,25(\mathrm{OH})_{2} \mathrm{D} 3$ in THP-1, dendritic cells and monocyte models. . . . . . . . . . . . . . 76

5.1 Antibodies used for flow cytometry assays . . . . . . . . . . . . . . . 104 

Chapter 1

\section{Introduction}




\subsection{History of vitamin D}

\subsubsection{Discovery of Vitamin D}

The early 1900s was an interesting period in the history of biochemistry when seminal studies revealed that unknown components in diet were vital for an organism's life. Most of these investigations focused on the study of diets that could potentially enhance development and growth, as well as preventing or healing common diseases. On a simplistic level, this resulted in the proposal that a balanced diet must contain $12 \%$ protein, $5 \%$ mineral, $10-30 \%$ fat to be considered a healthy diet [69]. This general nutritional philosophy continued until the early 1930s when the first experiments were carried out that led to the discovery of a new group of nutritional compounds referred to as "vitamins" [69]. In 1929 the Nobel Prize in Physiology or Medicine was given in equal parts to Christiaan Eijkman and Sir Frederick Gowland Hopkins "for the discovery of the growth-stimulating vitamins". In 1912, Frederick Hopkins published a study where he observed that rats fed with a diet purely consisting of proteins, carbohydrates, fat and salts caused an impairment in growth that was restored by the administration of a small amount of milk, suggesting that some basic nutrients present in a natural food (milk) were enough to fulfill the needs for an organism to grow normally [105]. Eijkman focused on the study of the health disorder beriberi in South East Asia where he carried out several animal experiments showing that the hull (or husks) of rice, which is usually removed in conventional polished rice, had properties that could cure beriberi. At the time these experiments, the concept of a vitamin did not exist but these initial studies were the starting points for further work that would reveal the importance of vitamins for normal human health.

The discovery of vitamins completely changed the perspective on diet and how dietary factors could be linked to diseases, leading to the study of how the deficiency of these basic compounds for life were causing impairments in the development and maintenance of organisms. It is worth mentioning that the two major contributions that led to the discovery of vitamin D were the work of Professor Elmer McCollum and Sir Edward Mellanby [57] (figure 1.1). McColumn was an American biochemist who focused his efforts on studying the impact that different components of food had on human health. His efforts to study the disorder xerophthalmia, concluded that a factor enriched in cod liver oil was crucial to the prevention of this disease [154]. Later on, this factor was identified as vitamin A [156]. Following McCollum's work, Sir Edward Mellanby was interested in the idea that rickets, a disease that was very common in Scotland, could be cured by the fortification/inclusion of specific components in food. To do so, Mellanby showed that he could cure rickets in dogs, who developed this bone disease through lack of light exposure, by feeding them with cod 


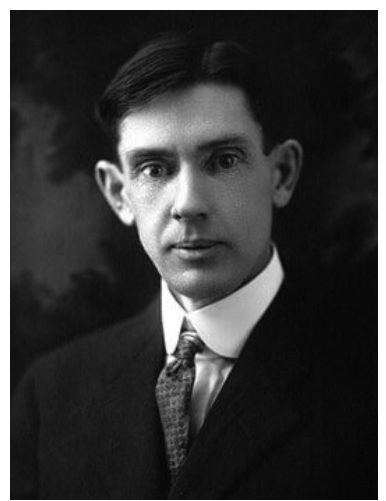

(a) Professor Elmer McCollum

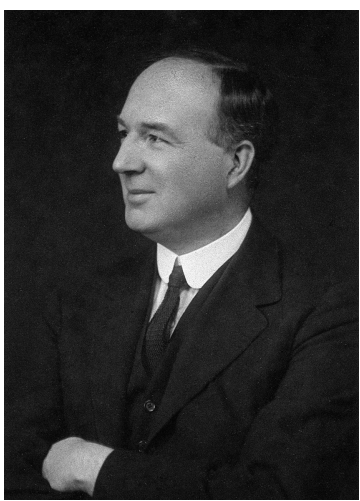

(b) Sir Edward Mellanby

Fig. 1.1 Major contributors who led to the discovery of vitamin D

liver oil. He wrongly assumed that the factor that cured rickets was vitamin A [158], but in 1922 McCollum, following Mellanby's observations, corrected this error. He observed that oxidized cod liver oil could not cure xerophthalmia nor vitamin A deficiency but nevertheless the capacity to cure rickets was retained. This led to their conclusion of the existence of a new vitamin involved in calcium homeostasis and bone health, namely vitamin D [155].

\subsubsection{Vitamin D deficiency}

In the mid 20th century, the main interest in vitamin D and human health was focused on its role in bone metabolism and mineral homeostasis, due to the link between vitamin D-deficiency and the disease rickets. However, the first actual scientific documentation of rickets dates back to the 17th century with the remarkable publications of Daniel Whistler and Francis Glisson describing a disease that affects children, and which is characterized by the incorrect mineralization of bone tissues resulting in the malformation of the skeletal structure [86] [87]. During the industrial revolution era, rickets in Europe was known as the "English disease" since an increased prevalence of young rickets populations was reported in several northern cities in England that mostly affected working-class families in developing urban areas. It was not until 300 years later that the discovery, isolation and study of vitamin D helped to understand how the deficiency of this factor could lead to dysregulation of mineral homeostasis and cause abnormal bone development. The most important discovery was that natural synthesis of vitamin D was due to either UV exposure of the skin [226], or ingest of specific foods such as milk and fatty fish that contained small amounts of vitamin D [159]. Because of these findings, in the mid 20th century the United Kingdom started to reinforce 
basic food, such as milk, with vitamin D to reduce vitamin D-deficiency and prevent rickets. However, this initial food fortification was stopped because of an increase in hypercalcemia cases in young people due to the inaccurate estimation of the dose needed to fortify food [229] [141]. Since then, there has been an ongoing debate about the potential advantages of adding vitamin D to foodstuff as a public health policy. In 2015 the UK Science Advisory Council on Nutrition (SACN) reported that, on a national level, it was impossible for UK residents to make adequate levels of vitamin D all year round, and they recommended that people take supplements or vitamin D-rich foods to raise serum vitamin D levels above a baseline serum level of $25 \mathrm{nmol} / \mathrm{L}$ [69]. In The Netherlands, addition of vitamins is regulated in specific foods such as "fat and baked and fried products"; in which vitamin D must be present up to 7.5-10 micrograms per 100 grams of the whole product (MVO - The Netherlands Oils and Fats Industry, https://www.mvo.nl/en-toevoeging-van-vitaminen-en-mineralen). In other parts of the world, notably North America, organisations such as the Endocrine Society and Institute of Medicine have recommended more active vitamin D supplementation to target higher, optimal levels of serum vitamin D of 75 [104] and $50 \mathrm{nmol} / \mathrm{L}$ respectively [55].

Although vitamin D-deficiency is a worldwide health problem, some populations appear to be at greater risk of low vitamin D status. This includes people with darker skin pigmentation living in Northern countries, and people who routinely cover up their skin - with epidermal synthesis of vitamin D being the major source of vitamin D for most people. Another population that is susceptible to vitamin D-deficiency is pregnant women since, among all the nutrients provided from the mother to the fetus, minerals are mainly used to build the fetal skeleton resulting in an increased demand of calcium and phosphates which is most predominant in the third trimester of the pregnancy [125]. Nevertheless, despite the fact that in pregnancy levels of the active form of vitamin D double the levels of a non-pregnant women [148], this increased concentration of the hormone is not linked to calcium homeostasis suggesting that vitamin D might play more roles in pregnancy. Bruce W Hollis et al [250] suggests that this might be linked to the immune tolerance needed during the generation of the fetus tissues. As I will discuss later, vitamin D plays an important role in the development of the immune tolerance system. Furthermore, more reports are emerging that link vitamin D deficiency with increased complications in pregnancy.

Vitamin D deficiency can be prevented and treated with healthy habits related to diet and UV-exposure [193]. Vitamin D can be taken from basic natural resources such as fish, mushrooms, eggs and butter. In most cases, and more frequently in low UV-exposure countries, vitamin D intake from natural food is insufficient and other strategies must be followed 
to promote vitamin D acquisitions. One strategy is to naturally fortify food with vitamin D; for example harvesting mushrooms that have grown under specific artificial UV lights or animal breed with food fortified with the vitamin. Furthermore, direct chemical food fortification for humans is another measure that many countries have followed to ensure the presence of certain levels of vitamin D in basic food such as milk, butter or cheese. Finally, the administration of vitamin $\mathrm{D}$ in the form of tablets is an efficient and modern achievement. It resulted from efforts in modern medicine to guarantee the intake of sufficient amounts of vitamin D by the most vulnerable sub-populations [69].

\subsection{Metabolism of vitamin D and tissue-specific actions}

As outlined above, vitamin D can be obtained either from a small number of dietary sources or by the action of sunlight on the skin. Irrespective of the initial source of vitamin D, the resulting vitamin $\mathrm{D}$ is not biologically active until after a series of key metabolic steps (figure 1.2). Keratinocytes within the skin contain the cholesterol-like molecule, 7-dehydrocholesterol (provitamin D3). It is this molecule that is photolytically converted to provitamin D3 when ultraviolet B (UVB) light enters the skin, with the resulting provitamin D3 molecule isomerizing to vitamin D3 (cholecalciferol). Some dietary sources contain another form of vitamin $\mathrm{D}$, vitamin D2 (calciferol), that is not found in animals but it has similar biological properties as vitamin D3. It is usually this vitamin D3 form that is referred to when describing vitamin $\mathrm{D}$ and its metabolites. Because vitamin $\mathrm{D}$ is a lipid-soluble molecule it is transported in the circulation primarily bound to proteins. This may include abundant proteins such as albumin that binds vitamin D with low affinity but, in addition, there is a dedicated vitamin D globulin known as vitamin D binding protein (DBP) which binds vitamin D with high affinity. 

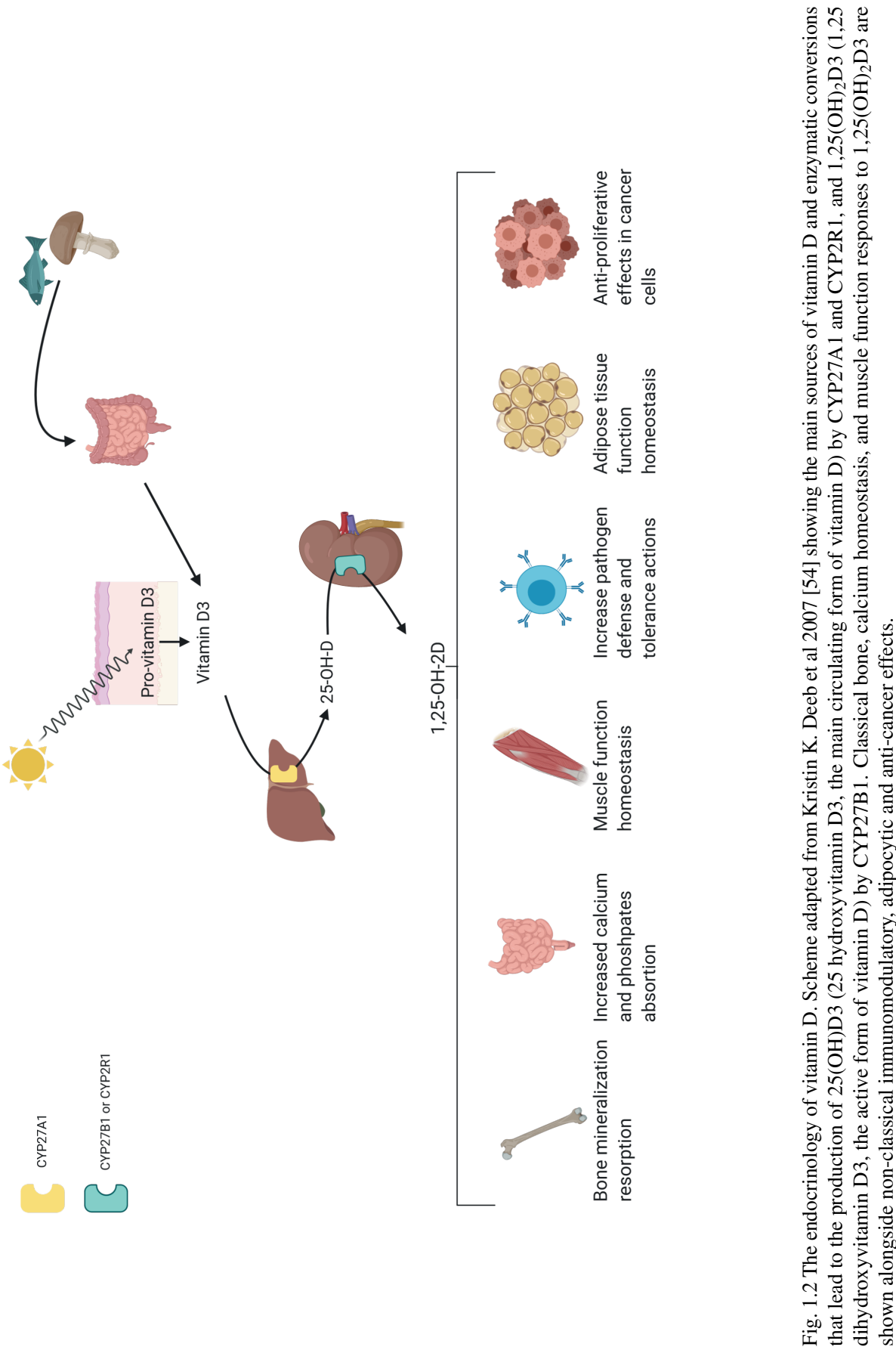
Vitamin D has a short half-life and is rapidly transported to the liver for the first of several metabolic conversion steps. The enzymes involved in this metabolism belong to the family of cytochrome P450 enzymes (CYP) that are divided into two main groups depending on their cellular location: microsomal and mitochondrial; both are codependent on electron transport chain proteins [69]. The first enzymes that metabolize vitamin D are the microsomal and mitochondrial D-25-hydroxylase enzymes CYP2R1 and CYP27A1 respectively which are mainly expressed in liver cells and which convert vitamin D to 25-hydroxyvitamin D $(25(\mathrm{OH}) \mathrm{D})$. This inactive form of vitamin $\mathrm{D}$ is the most abundant in blood and has the highest binding affinity for DBP. Membrane receptors of renal proximal tubular cells recognise the $25(\mathrm{OH}) \mathrm{D}-\mathrm{DBP}$ complex which is then taken up by these cells and metabolized by another CYP, 25-hydroxyvitamin D-1 $\alpha$-hydroxylase (CYP27B1). The resulting product of the $1 \alpha$ hydroxylation of $25(\mathrm{OH}) \mathrm{D}$ is 1,25 -dihydroxyvitamin $\mathrm{D}\left(1,25(\mathrm{OH})_{2} \mathrm{D} 3\right)$, the active form of vitamin $\mathrm{D}$ that is commonly referred to as a steroid hormone [69]. There are two reasons for $1,25(\mathrm{OH})_{2} \mathrm{D} 3$ is referred to as a hormone. Firstly, the major actions of $1,25(\mathrm{OH})_{2} \mathrm{D} 3$ are endocrine, in that it is produced by the kidneys and then acts in very small concentrations on distal tissues such as the gastrointestinal tract (to enhance mineral uptake from diet), and parathyroid glands (to suppress synthesis of parathyroid hormone, PTH) [34]. In the kidneys, PTH is the major stimulator of $1,25(\mathrm{OH})_{2} \mathrm{D} 3$ production via CYP27B1 [75], so this also represents endocrine feedback control. The second reason $1,25(\mathrm{OH})_{2} \mathrm{D} 3$ is considered a hormone is that its actions are mediated in a similar fashion to other steroid hormones such as estrogens, androgens, and corticosteroids, namely by binding to a specific nuclear receptor - the vitamin D receptor (VDR) [117]. The intracellular complex of $1,25(\mathrm{OH})_{2} \mathrm{D} 3$ bound to VDR acts as a transcription factor that binds with a heterodimer partner (retinoid $\mathrm{X}$ receptor, $\mathrm{RXR}$ ) to gene promoter vitamin D response elements (VDRE) DNA sequences to regulate chromatin structure and up- or down-regulate transcription of target genes [95][36]. Because VDR is expressed in many cell types throughout the body, it is possible that $1,25(\mathrm{OH})_{2} \mathrm{D} 3$ can influence the function of many tissues. Further to the gene regulation capacity of the $1,25(\mathrm{OH})_{2} \mathrm{D} 3-\mathrm{VDR}$ axis, it is important to recognise that although the classical transcriptional response to $1,25(\mathrm{OH})_{2} \mathrm{D} 3$ is mediated via binding to VDR and heterodimerisation with the retinoid $\mathrm{X}$ receptor $(\mathrm{RXR}), 1,25(\mathrm{OH})_{2} \mathrm{D} 3$ may also achieve effects via other pathways. In addition to that, it has been demonstrated that VDR functionalizes as homodimer [215]. Similarly, $1,25(\mathrm{OH})_{2} \mathrm{D} 3$ also appears to achieve rapid effects via membrane-mediated effects through phospholipase $\mathrm{A}_{2}$ activating protein (PLAA) and $\mathrm{Ca}^{2+} /$ calmodulin-dependent kinase II (CaMKII) axis signalling pathway [62]. The precise receptor associated with membranemediated, non-genomic, effects of $1,25(\mathrm{OH})_{2} \mathrm{D} 3$ has yet to be full-defined but it is known that $1,25(\mathrm{OH})_{2} \mathrm{D} 3$ is able to regulate the activity of a wide range in intracellular kinases 
enzymes, including phosphoinositide 3-kinase (PI3K), protein kinase $\mathrm{C}$ (PKC), extracellularsignal-regulated kinase (ERK) and c-Jun N-terminal kinases (JNKs). A schematic pathway diagram was created in WikiPathways which compiles all non-genomic actions of vitamin D [102] (https://www.wikipathways.org/index.php/Pathway:WP4341). In this thesis, we will focus on the ways in which $1,25(\mathrm{OH})_{2} \mathrm{D} 3$ and VDR can function to regulate immune activity in cells from both the innate and adaptive immune system.

The active form of vitamin $\mathrm{D}$ is well known as being a crucial hormone for mineral homeostasis in the organism as $1,25(\mathrm{OH})_{2} \mathrm{D} 3$ stimulates the absorption of minerals in intestine cells and reabsorption of bone increasing the levels of calcium and phosphates in the bloodstream [45]. Further studies of the role of vitamin D in extra-skeletal tissues revealed that deficiency of this vitamin was linked to several pathologies affecting different tissues. For instance, recent studies have shown that vitamin D deficiency is correlated to muscle weakness and increased risk of falls [92]. There has also been increased interest in vitamin D metabolism in adipose tissue since studies have correlated metabolic disorders, such as obesity, with vitamin D homeostasis [181], demonstrating that adipose tissue responds to and is involved in vitamin D metabolism [58]. In addition to that, recent studies showed that obese population have lower levels of vitamin D suggesting these patients need vitamin D supplementation [251]. Furthermore, studies in the last two decades have explored the anti-proliferative properties of vitamin D in malignant neoplastic cells [54][130]. Notably, two studies (one of them discussed in Chapter 4) carried out a similar pathway analysis that described in this thesis and showed that $1,25(\mathrm{OH})_{2} \mathrm{D} 3$ represses gene expression of cell cycle components in prostate and myeloid cancer cell lines [132][81]. Finally, throughout this thesis we will explain in more detail the role of vitamin D in the immune system, showing that its different immunomodulatory roles, depending on immune cell type, have the potential to generate a specific immune response. To do that, we will introduce in the following section the most important aspects of the immune system and their interaction with vitamin D.

\subsection{Immune system}

\subsubsection{Myeloid cells}

The myeloid cell lineage (figure 1.3) includes red blood cells, such as erythrocytes that deliver oxygen to different tissues, and white blood cells, specialized innate immune cells responsible for the first host barrier defense. The second group fulfills the main innate immune functions and is considered the first front line of defense against infections and is 
also responsible to deliver the activating signal to the lymphoid cells to promote the adaptive immune response. Myeloid progenitor cells can develop into cells that can be subdivided into granulocytes and antigen-presenting cells (APC).

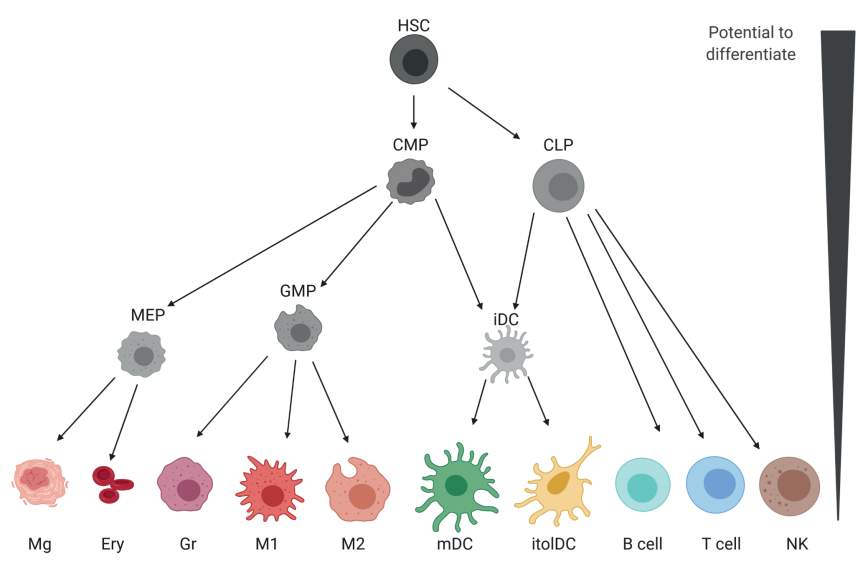

Fig. 1.3 Schematic representation of hematopoietic stem cell (HSC) development. Scheme adapted from Weiskopf K et al 2017 [256]. Hematopoietic stem cell (HSC) can develop to the common myeloid progenitor (CMP) which can give rise to the megakaryocytes $(\mathrm{Mg}) /$ erythrocyte (Ery) progenitor (MEP) leading to the formation of thrombocytes and red blood cells respectively. CMP can develop to the granulocyte (Gr)/ macrophage (M1-2) progenitor (GMP) but also can develop to immature dendritic cells (iDC). Some iDC can rise from common lymphoid progenitor cells (CLP) and then differentiate to specific DC such as mature (mDC) or tolerogenic (tolDC). CLP generally give rise to $\mathrm{B}$, T and NK cells.

\section{Granulocytes}

Granulocytes are a group of myeloid cells that are characterised by a multilobed nucleus and the presence of vesicles called granules in their cytoplasm containing anti-pathogen proteins and cytokines that will be liberated to the extracellular medium in response to an infection. Within the granulocyte group, cells can be sub-classified as eosinophils, basophils, mast cells and neutrophils. The most characteristic subgroup is the neutrophils since they are the most abundant white blood cells in circulation (up to 70\%). Neutrophils, after differentiating in the bone marrow, start circulating as a cell patrol to rapidly respond to infections and phagocytose extracellular bacterial and fungi pathogens for subsequent killing within specialised organelles known as phagosomes [29]. Neutrophils have developed several mechanisms to undertake actions against pathogens. After the migration to the place of infection they are capable of liberating cytokines that promote the recruitment of other immune cells and directly phagocyte extracellular pathogens for their elimination [165]. 


\section{Antigen-presenting cells (APCs)}

APCs are a group of myeloid cells that are able to recognise pathogens and to present pathogen material to other immune cells. For these cells, first contact with a pathogen activates signalling pathways that stimulate APC innate host-defense mechanisms whilst also recruiting other cells to the place of infection. The ability of APC to phagocytose hostile organisms and degrade the resulting internalized organism into antigens for presentation to lymphoid cells forms the basis of adaptive immune responses, and the development of immune memory. From myeloid progenitor a more specialized cell named monocyte is generated that can develop in two major APC types: macrophages and dendritic cells (DC), and these are discussed in further detail below.

\section{Macrophages}

Monocytes differentiated in the bone marrow from the common myeloid progenitor (CMP) are released into the bloodstream to come part of the larger population of peripheral blood mononuclear cells (PBMCs), with subsequent differentiation, under specific inflammation conditions, to macrophages. Most of adult macrophages are generated during embryonic development, and depending on the target tissue where they settle, they are specialized in macrophage-like cells such as osteoclasts in bones, microglia cells in the nervous system or alveolar macrophages in the lung [65]. Thanks to their plasticity at the functional level, these cells are crucial for tissue homeostasis, precursors and modulators of inflammation and bridge between innate to the adaptive immune system [169] [245]. Here we will exclusively focus on their innate immune function.

The process of inflammation is a complex response that can be summarized in a few steps: isolation of the infection site, blood vessel permeabilization, cell recruitment, pathogen/infected cell removal, tissue repair and resolution of inflammation. These different processes are orchestrated by the action of a diverse array of cells, and a similar diverse array of cytokines and chemokines that regulate each step of the inflammatory immune response. Proinflammatory macrophages, commonly named as M1-macrophage, are characterized by the high expression of markers that promote anti-pathogen responses such as pattern-recognition receptors (PRR) such as toll-like receptors (TLR) that recognise pathogen-associated molecular patterns (PAMPs) [19]. The resulting PAMP-PRR interaction promotes phagocytosis of the pathogen and its subsequent destruction in specialised macrophage phagosomes. The resulting breakdown of the pathogen enables presentation of antigen by macrophages to lymphoid cells via the major histocompatibility complex II (MHC-II) stabilized by CD80 
and CD86 receptors promoting the adaptive immune response. M1-macrophages are also characterized by the liberation of chemokines and cytokines to stimulate and recruit cells to the site of inflammation [13]. As outlined above, the process of inflammation it is also characterised by tissue repair and the eventual resolution of inflammation. There are several types of macrophages that have an anti-inflammatory profile and which are broadly grouped as M2-macrophages [108], that are characterized by the production of anti-inflammatory cytokines such as IL-4, IL-10 and TGF- $\beta$ [109][10].

\section{Dendritic cells (DCs)}

Dendritic cells are bone-marrow derived cells which can derived from CMP and common lymphoid progenitor (CLP); of which each has a well-defined role in immunity. DC development can lead to various subtypes depending on the stimulation received and the specific tissue microenvironment [51]. In this way DC can stimulate a range of immune responses from the activation of adaptive immune response to pathogen infection [237] or the presence of tumour cells [82], to the promotion of more tolerogenic immune actions in situations such as organ/cell transplantation [228]. The DC phenotype plasticity level enhances the ability of these cells to modulate the immune response in different immune contexts. However, in the current study we have focused on monocyte-derived DC, which their development is achieved under inflammatory conditions [51], and have contrasted the role of DC in response to a pathogen stimulus relative to a more tolerogenic role for DC in an anti-inflammatory setting.

Immature DCs (iDCs) are present in the vast majority of tissues and, as APC, they have the capacity to recognise and process pathogens and present the resulting antigens to naïve $\mathrm{T}$ ( $\mathrm{Tn}$ ) cells leading to the activation of adaptive immune responses. When exposed to maturation effectors such as bacterial cell wall component lipopolysaccharide (LPS), or the inflammatory cytokine interferon $\gamma(\mathrm{INF}-\gamma)$, iDCs develop a more mature DC (mDC) phenotype characterized by an increased capacity to migrate to lymphoid organs, and expression of $\mathrm{T}$ cell activation effectors. The development of $\mathrm{mDC}$ is characterised by expression of maturation markers such as CD80, CD86 and MHC-II, membrane proteins that promote DC-T cell interaction and antigen presentation respectively, leading to subsequent $\mathrm{T}$ cell activation [142]. Antigens presented by DC through MHC-II are recognized by T cell receptors (TCR), this interaction is stabilized by the DC proteins CD80/CD86 that interact with the membrane $\mathrm{T}$-cell receptor $\mathrm{CD} 28$, leading to a signaling cascade that promotes $\mathrm{T}$ cell activation.

In contrast to an antigen challenge, specific stimuli, such as the cytokines IL-27 or IL-10, or 
Table 1.1 Main immune cell models that will be studied in higher detail in this thesis. This table summarizes the key immune cell features describe in in vitro models. Description of DC features are focused on monocytederived DC. [170] [212][210][1][180][204][60]

\begin{tabular}{|c|c|c|c|c|}
\hline Cell type & Surface markers and nuclear receptors & Cytokines production & Factors induce their development & Main function \\
\hline M1 & $\begin{array}{l}\text { MHCII high } \\
\text { CD80 high } \\
\text { CD } 86^{\text {high }}\end{array}$ & $\begin{array}{l}\text { IL-6 } \\
\text { IL-12 } \\
\text { IL-23 }\end{array}$ & $\begin{array}{l}\text { LPS } \\
\text { INF- } \gamma \\
\text { GM-CSF } \\
\text { Intracellular pathogens } \\
\text { Bacterial components }\end{array}$ & $\begin{array}{l}\text { Pathogen defence, } \\
\text { Pro-inflammatory actions, } \\
\text { Antigen presentation }\end{array}$ \\
\hline M2 & $\begin{array}{l}\mathrm{MHCII}^{\text {high }} \\
\mathrm{CD}^{\text {high }}\end{array}$ & $\begin{array}{l}\text { IL-4 } \\
\text { IL10 } \\
\text { TGF- } \beta\end{array}$ & $\begin{array}{l}\text { IL-4 } \\
\text { Fungal cells } \\
\text { Parasites }\end{array}$ & $\begin{array}{l}\text { Tissue repairment } \\
\text { Anti-inflammatory actions } \\
\text { Resolution of inflammation }\end{array}$ \\
\hline iDC & $\begin{array}{l}\text { CD14 } \\
\text { CD11c } \\
\text { CD209 }\end{array}$ & $\begin{array}{l}\text { IL-6 } \\
\text { IL-12p70 } \\
\text { IL-23 }\end{array}$ & $\begin{array}{l}\text { GM-CSF } \\
\text { IL-4 }\end{array}$ & Plasticity development \\
\hline mDC & $\begin{array}{l}\text { MHCII }{ }^{\text {high }} \\
\text { CD80 } \\
\text { CD } 86^{\text {high }} \\
\text { CD209 }\end{array}$ & $\begin{array}{l}\text { IL-27 } \\
\text { IL-6 } \\
\text { IL-12p70 } \\
\text { IL-23 }\end{array}$ & $\begin{array}{l}\text { LPS } \\
\text { INF- } \gamma\end{array}$ & $\begin{array}{l}\text { Pathogen defence } \\
\text { Antigen presentation } \\
\text { Pro-inflammatory actions }\end{array}$ \\
\hline itolDC & $\begin{array}{l}\text { ILT-3 \& ILT-4 } \\
\text { CD11c } \\
\text { MHCII low } \\
\text { CD80 } \\
\text { CD86 } \\
\text { low }\end{array}$ & IL-10 & $\begin{array}{l}\text { IL-27 } \\
\text { IL-10 } \\
\text { Corticosteroids } \\
\text { Vitamin D }\end{array}$ & $\begin{array}{l}\text { Treg development } \\
\text { Anti-inflammatory actions }\end{array}$ \\
\hline Th1 & $\begin{array}{l}\text { CD4 } \\
\text { CXCR3 } \\
\text { STAT4 } \\
\text { AP-1 }^{\text {low }} \\
\text { NFAT }^{\text {low }}\end{array}$ & $\begin{array}{l}\text { TNF } \\
\text { INF- } \gamma\end{array}$ & $\begin{array}{l}\text { INF- } \gamma \\
\text { IL-12 } \\
\text { IL-18 }\end{array}$ & $\begin{array}{l}\text { Promotes APC function } \\
\text { Promotes Tc activation } \\
\text { Intracellular pathogen defence }\end{array}$ \\
\hline Th2 & $\begin{array}{l}\text { CD4 } \\
\text { CCR4 } \\
\text { AP-1 }^{\text {high }} \\
\text { NFAT }^{\text {high }} \\
\text { GATA-3 } \\
\text { c-maf } \\
\text { STAT6 }\end{array}$ & $\begin{array}{l}\text { IL-4 } \\
\text { IL-5 } \\
\text { IL-13 }\end{array}$ & $\begin{array}{l}\text { IL-4 } \\
\text { IL-23 }\end{array}$ & Extracellular pathogen defence \\
\hline Treg & $\begin{array}{l}\text { CTLA-4 } \\
\text { Foxp3 }\end{array}$ & $\begin{array}{l}\text { IL-4 } \\
\text { IL-10 }\end{array}$ & $\begin{array}{l}\text { I-6 } \\
\text { IL10 } \\
\text { IL-21 }\end{array}$ & $\begin{array}{l}\text { Enhances tolerance } \\
\text { Anti-inflammatory actions }\end{array}$ \\
\hline NK cells & $\begin{array}{l}\text { Fas ligand (FASL) } \\
\text { IL-2R } \\
\text { CD56 }\end{array}$ & $\begin{array}{l}\text { Perforins } \\
\text { INF- } \gamma \\
\text { TNF }\end{array}$ & $\begin{array}{l}\text { IL-2 } \\
\text { IL-7 } \\
\text { IL15 }\end{array}$ & Induce apoptosis in anomalous cells \\
\hline
\end{tabular}

treatment with corticosteroids or $1,25(\mathrm{OH})_{2} \mathrm{D} 3$ [152][167][153] can induce anti-inflammatory DC populations characterised by their capacity to suppress $\mathrm{T}$ cell effector responses and promote the development of T regulatory (Treg) cells. This type of DCs is commonly referred to as tolerogenic DC (tolDC) and their phenotype is characterised by the reduction of CD80 and CD86, decreased production of pro-inflammatory cytokines [227] and enhanced production of anti-inflammatory cytokines such as IL-10 [40][200]. 


\subsubsection{Lymphoid cells}

Lymphoid cells are characterised by their ability to produce a more specific response to pathogens based on their response to antigens presented by APC. There are three main groups of lymphoid cells (figure 1.3): B cells, T cells and natural killer (NK) cells. Morphologically these cells are hard to distinguish but they can be defined by specific cell surface cluster designation (CD) markers. Broadly speaking, lymphocytes can act as effector cells in primary responses when they recognize antigen for the first time, triggering adaptive immune responses to counteract infection. Otherwise, lymphoid cells exist in a resting state until subsequent exposure to a specific antigen facilitates faster adaptive immune responses. These cells are known as memory $\mathrm{T}$ cells.

\section{T cells}

Lymphoid precursor cells that arise in the bone marrow travel as PBMCs through the bloodstream to the primary lymphoid organ known as thymus. Here, immature T cells, known as thymocytes, undergo complex cell differentiation into different $\mathrm{T}$ cell subtypes. In particular, all T cells undergo two checkpoints that will determine their correct development: 1) $\mathrm{T}$ cells that can recognise self MHC are selected to survive (positive selection); 2) T cells that interact with high affinity to self-antigens are selectively "killed" (negative selection). With this selection mechanism the lineage of $\mathrm{T}$ cells generated through this process guarantees effective recognition of, and response to, foreign antigens presented by the host APC. T cells can then start an effector immune response whilst avoiding generation of $\mathrm{T}$ cells reactive to host components. Broadly speaking, this maturation of $\mathrm{T}$ cells result in the generation of three types of T cells: 1) T helper (Th) cells; 2) T cytotoxic (Tc) cells; 3) Treg cells.

\section{T helper vs cytotoxic $\mathbf{T}$ cells}

Th cells can be considered as assistance cells needed for optimal immune response to infection. Within this group, Th1 and Th17 cells constitute a subgroup of Th cells that enhances and modulates inflammatory immune cell-mediated responses including APC and Tc activation in response to extracellular pathogens (restricted to bacteria and fungi) [147]. Th2 and follicular T cells (Tfh) are involved in modulation of antibody (humoral) immune defense that includes defense against more complex organisms such as parasitic worms (helminths) [147]. In contrast, T cytotoxic cells are involved in the direct induction of cell death and liberation of proinflammatory signals in response to infection [267]. 


\section{$\mathrm{T}$ regulatory cells}

The major responsibility of the thymus is to provide an optimal environment for $\mathrm{T}$ cell development and effector immune responses to specific pathogen challenges. The thymus also functions to generate the machinery needed for maintenance of self-tolerance - in other words, generation of a subpopulation of T cells that does not attack the host. Whereas Th and Tc cells function as effector immune cells, promoting immune response, Treg cells are involved in anti-inflammatory responses that suppress inflammation. Treg are a subpopulation of CD4+ T cells characterized by high expression of Foxp3, CTLA-4, IL-2 receptor and CD25 markers. As G.Plitas and A. Rudensky explains in their review [195], the homeostatic properties of Treg are able to repress the actions of various types of effector cells. For instance, a major characteristic of Treg is production of the tolerogenic cytokine IL-10, whose functions include the suppression of Th17 cell activity in inflammation [40]. Treg are also able to down-regulate expression of mature markers of DC such as CD80 and CD86 through signaling induced by the T cell surface protein CTLA-4 [260]. Furthermore, it has been shown that high expression of IL-2 receptor by Treg is crucial in limiting the availability of IL2, essential interleukin needed for activation of other T cell groups such as CD8+ cells [42]. Ultimately, Treg are essential for the control and resolution of immune responses, and failure in their development or functionality can lead to dysregulation of the immune system resulting in autoimmune disorders.

\section{NK cells}

The vast majority of NK cells develop in very specific niches from the CLP in the bone marrow [1] but recent studies suggest that NK cells also develop in secondary lymphoid organs such as the spleen [71]. When they are fully differentiated they travel through the bloodstream providing 5-15\% of the total PBMC population in adult humans [71]. Interestingly, this class of lymphoid cells seems to be crucial for the development of the decidual tissue that facilitates the connection between the fetus and the mother early in pregnancy, where NK cells make up 50-90\% of the whole population of lymphoid cells located at the maternal-fetal interface [120] [31]. The classical role of NK cells is to recognize cells that are malfunctioning due to pathogen infection, or cells that have undergone development into cancer. The direct mechanism in which NK cells kill defective cells is by the liberation of specific proteins known as granzymes, proteases that activate apoptosis programming that induces DNA fragmentation, and perforins, monomers that once liberated form a pore in the target cell promoting cell apoptosis. Finally, NK cells can induce cell-death via the expression of Fas ligand (FasL), that when it interacts with its receptor Fas, that is over-expressed in 
malfunctioning cells [187], induces a signaling apoptotic pathway. Interestingly, the NK cells that are abundant in the decidua (also known as uterine NK cells) have less cytotoxic innate immune actions than blood NK cells, and instead appear to facilitate the arterial remodelling that is crucial for placental development [209].

\section{B cells}

Lymphoid cellular responses are mostly carried out by $\mathrm{T}$ and NK cells where they mediate direct actions to promote the elimination of pathogens or damaged cells, and to modulate other immune cells via the release of specific cytokines. Other immune responses do not depend on direct actions of immune cells but, instead, involve the production and release of antibodies as part of the humoral immune response. These cells are B cells.

The progenitor of B cells is the CLP that resides in the bone marrow, and B cell development takes place in the bone marrow and secondary lymphoid organs such as the spleen, resulting in the generation of memory B cells and plasma cells [103]. B cells express the transmembrane protein known as B cell receptor (BCR) that, after specifically recognising a specific antigen, will start a signaling cascade that will determine B cell activation. Furthermore, mature B cells are capable of producing different immunoglobulins, also known as antibodies: $\operatorname{IgA}, \operatorname{IgD}, \operatorname{IgE}, \operatorname{IgG}$ and $\operatorname{IgM}$. Once released, these immune molecules can bind directly to the pathogen and neutralize it directly, avoiding any interaction with the host and tagging it for its elimination. Alternatively, antibodies can promote the formation of the complement system in order to kill the pathogen by its lysis or the induction of cell-mediated elimination [103]. Finally, inactive B cells, once they interact with a specific antigen undergo into an activation process that is characterised by enhance $B$ cell proliferation and increase secretion of specific antibodies. The initials antibodies generated in B cells will be located in the cell membrane serving as a membrane receptor for the specific antigen [8].

\subsection{Role of vitamin $D$ in different immune cell types}

The immune system of higher organisms has evolved to an interconnected complex of specialized tissues, cells and molecules which work together to defend the host from different kinds of extracellular and intracellular pathogens, as well as challenges by damage-associated molecules from the host itself. The idea that vitamin D could play a role in regulating innate and/or adaptive immunity stems from two observations that were made more than 30 years ago: 1) that many cells from the immune system express VDR [20] [198] [128] [233] [28][150]; 2) that, under specific conditions antigen-presenting cells such as macrophages 
have the ability to synthesize $1,25(\mathrm{OH})_{2} \mathrm{D} 3$ from 25OHD3 [264]. These initial observations have since been expanded to include possible effects of vitamin D on many cells from the immune system as outlined below (figure 1.4).

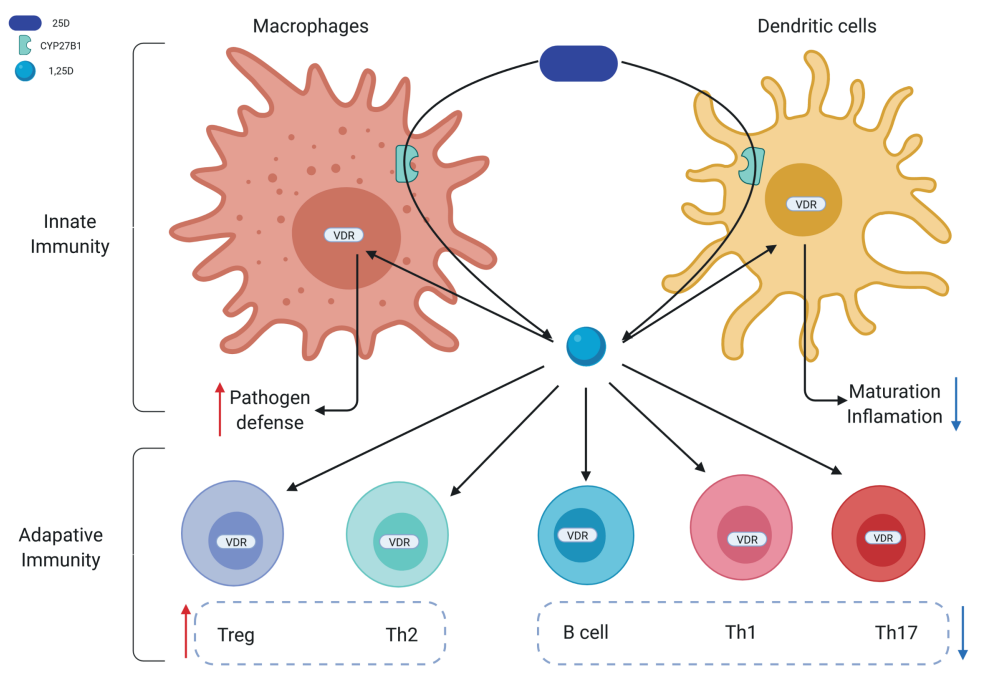

Fig. 1.4 Autocrine and paracrine actions of vitamin D in innate immune cells and its effects on adaptive immune cells. Macrophages and dendritic cells express CYP27B1, capable to synthesise $1,25(\mathrm{OH})_{2} \mathrm{D} 3$ from $25(\mathrm{OH}) \mathrm{D}$. The active form of vitamin $\mathrm{D}$ regulates gene expression in cells that express vitamin $\mathrm{D}$ receptor (VDR) stimulating or inhibiting immune actions depending on the cell type.

\subsubsection{Myeloid cells and vitamin D}

The initial link between vitamin $\mathrm{D}$ and myeloid cells was the observation that $1,25(\mathrm{OH})_{2} \mathrm{D} 3$ can act on monocytes expressing the VDR to inhibit their proliferation [56]. However, more recent studies have focused on the role of vitamin D in enhancing the antibacterial properties of myeloid cells such as monocytes and macrophages [98]. The molecular mechanism by which $1,25(\mathrm{OH})_{2} \mathrm{D} 3$ promotes antibacterial responses involves the activation of CYP27B1 and VDR expression following immune challenge [145], and the subsequent binding of $1,25(\mathrm{OH})_{2} \mathrm{D} 3$-bound VDR to DNA VDRE in the gene promoters of antibacterial factors such as cathelicidin and $\beta$-defensin 4A (DEFB4A) [254]. These responses appear to be mediated by pathogen pattern recognition by Toll-like receptors 1 and 2 (TLR-1 and TLR-2)[254], which promotes enhanced expression of CYP27B1 in macrophages and DCs, suggesting that some myeloid-derived cells are capable to synthesize locally $1,25(\mathrm{OH})_{2} \mathrm{D} 3$ and, in this way, promote and intracrine/paracrine antibacterial responses to the pathogen [145]. This 
antibacterial effect of $1,25(\mathrm{OH})_{2} \mathrm{D} 3$ is particularly important for intracellular pathogens such as Mycobacterium tuberculosis (M. tb), with $1,25(\mathrm{OH})_{2} \mathrm{D} 3$ also promoting autophagy to enhance the autophagosomal environment in which $\mathrm{M}$. Tb can be killed [33].

In contrast to its positive impact on antibacterial responses, vitamin $\mathrm{D}$ also promotes antiinflammatory responses in macrophages and DC, consistent with its tolerogenic function [91]. Tolerance is a part of the immune system that aims to recognise structures that are from the host versus those that are from non-host components. Failure of this system may lead to autoimmune diseases that are characterised by the activity of immune cells towards host cells and tissues. The correct development of specific cells is needed for the maintenance of self-tolerance. In particular, this requires coordinated actions of DC and T cells. TolDCs are a subtype of DCs where maturation has been suppressed, resulting in lower expression of CD80/CD86 and MHCII thereby modulating the ability of these cells to promote CD4+ effector $\mathrm{T}$ cell activation. Tolerogenic effects of vitamin $\mathrm{D}$ in $\mathrm{DC}$ are characterised by the suppression of the expression of CD80/CD86 and MHCII and enhanced expression of tolerogenic markers such as IL-10, IL-27 and CD11c [186]. Since IL-10 induces anergy in T effector cells and promotes differentiation of Treg [89], vitamin D-induced tolDC may be crucial in the maintenance of immune tolerance.

\subsubsection{Lymphoid cells and vitamin D}

As well as modulating T cell function through indirect effects on DC phenotype mentioned above, $1,25(\mathrm{OH})_{2} \mathrm{D} 3$ can also act directly on $\mathrm{T}$ cells and B cells expressing VDR. Interestingly, resting, non-activated $\mathrm{T}$ cells and $\mathrm{B}$ cells have low or undetectable levels of VDR, which is only significantly expressed following immunogenic activation with the onset of lymphocyte proliferation [21] [198]. Studies have shown that overall $1,25(\mathrm{OH})_{2} \mathrm{D} 3$ has a negative effect on proliferation in T cells [203] and inhibits the proinflammatory response of Th1 and Th17 [35] [116] populations, whilst promoting the anti-inflammatory Th2 [26] and Treg populations [110]. Finally, studies have shown that $1,25(\mathrm{OH})_{2} \mathrm{D} 3$ has similar anti-proliferation effects in $\mathrm{B}$ cell populations as observed in $\mathrm{T}$ cells, and also decreases the capacity for plasma cell differentiation and reduces the Ig production [41].

For the final group of lymphoid cells, NK cells, the effects of vitamin D are much less well understood. NK cells have the same lymphoid progenitor as $\mathrm{T}$ and $\mathrm{B}$ cells but no antigen receptors and also are considered part of the innate immune system. As their name suggests, NK cells are specialized in the "killing" of malfunctioning cells such as tumors or infected cells [184]. This is based on sensing MHC-I levels in the target cells to establish a tight 
interaction, and the subsequent induction of apoptosis in the target by the release of FasL and granules that contain perforin and granzyme proteases to enable cell lysis. However, NK cells can also function as part of normal, healthy, physiology. In pregnancy, it has been shown that NK cells from the maternal uterus (uNK) play a key role in maintaining the healthy development of the maternal-fetal interface. This tissue-specific type of NK cells has important non-cytotoxic roles in processes such as placental vascular formation and cell factor production [224]. The importance of uNK in maintaining normal pregnancy is illustrated by recent studies showing a role for uNK dysfunction in recurrent pregnancy loss (RPL) [78]. Relatively little is known about the impact of vitamin D in NK cells in general, but studies have shown regulatory effects of $1,25(\mathrm{OH})_{2} \mathrm{D} 3$ on uNK [178] that may help to explain the possible link between vitamin D-deficiency and RPL [177].

\subsection{Functional genomics analysis}

The role of vitamin D as a modulator of gene expression in immune cells has been studied in detail thanks to genomic analysis [47]. Large scale gene expression datasets from different immune cells treated with different forms of vitamin D have been generated in the last decade, contributing to the understanding of the transcriptomic impact of vitamin D on the immune system. However, the generation of this kind of data is considered only the first step in a larger journey of understanding of how a biological system responds to a particular stimulus. Further analysis is required to interpret the large datasets created by transcriptomic or proteomic analyses, and this approach is broadly referred to as 'Bioinformatics'. The field of Bioinformatics is a biomedical science in which computational techniques are created to simplify and shorten large-scale data processing and analysis. Here, we explain how different bioinformatics tools can be used to statistically analyse and biologically contextualize transcriptomic datasets in the setting of different aspects of molecular biology in a user-friendly way via the following steps: 1) retrieving transcriptomic data for qualitycontrol analysis; 2) identification of biological pathways altered by a particular treatment or disease based on the dataset; 3 ) network expansion of identified biological pathways with molecule-molecule interactions using additional databases; 4) visualization and interpretation of the results (figure 1.5).

\subsubsection{Biological databases}

Biomolecular data and knowledge is stored in biological databases serving as libraries that compile experimental work from different molecular fields of biomedical science, creating 


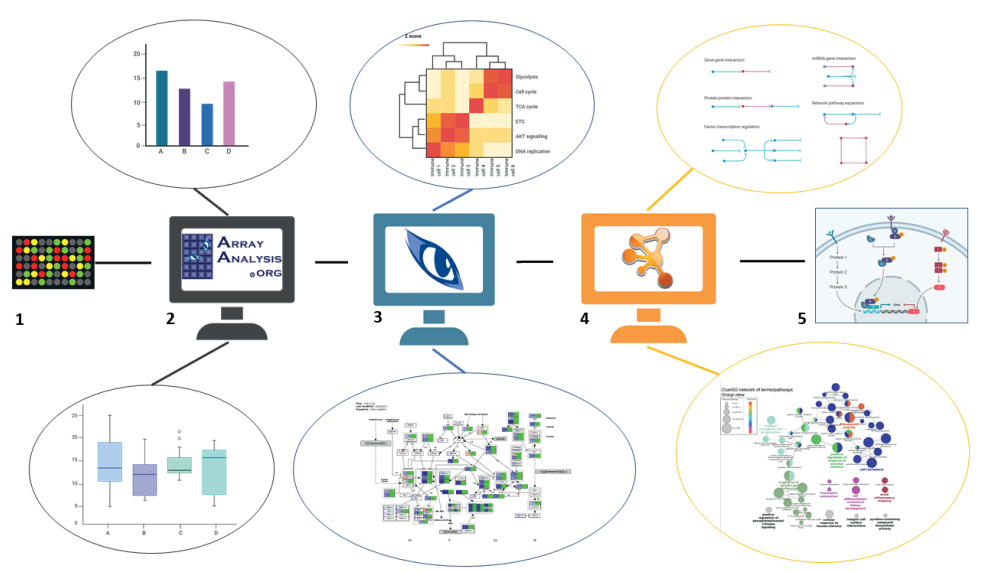

Fig. 1.5 The application of bioinformatic tools for analysis of transcriptomic datasets. Schematic representation of the bioinformatic pipeline used in this project to analyse raw gene expression data and identify key pathways affected by $1,25(\mathrm{OH})_{2} \mathrm{D} 3$. 1) Quality of unprocessed raw gene expression data was analysed and normalized using ArrayAnalysis web-tool. 2) Normalized and processed data was used to identify biological pathways altered based on the statistical parameters using PathVisio. 3) Cytoscape was used to analyse a subset of genes to identify enriched gene ontology terms and extend biological network with gene-molecule interactions such as miRNA. 5) The interpretation of the results gave new light into the role of vitamin $\mathrm{D}$ in the immune biological system.

several specialized repositories that contain information about the nature, reaction and interaction of biomolecules. Open databases make possible the usage of this information to exploit datasets at many biological levels, providing additional puzzle pieces to a whole picture of a system. In this PhD project, experimental datasets were used as the first pillar of a network, with this pillar being extended by pathway analysis using molecular information from different databases to build an expanded biological network that allowed us to better understand how the individual components of a biological system interact. In this particular study, analysis of gene expression public datasets using pathway analysis strategies identified pathways that were significantly altered by treatment with $1,25(\mathrm{OH})_{2} \mathrm{D} 3$ in different immune cell types. This analysis could then be visually represented by tools such as Cytoscape to provide a further layer of information from the data stored in external public repositories.

Previous studies have reported that vitamin D also affects the expression of specific microRNAs (miRNA), thereby influencing post-transcriptional regulation of gene expression in target cells. Therefore in our analysis, we also linked information from miRTarBase [44] and miRbBase [127] to study the potential regulation between the subsets of genes identified in the transcriptomic analyses, and the miRNAs that they interact with. In this way, functional genomic experiments assessing the impact of vitamin D on miRNA and mRNA expression 
in immune cell models can be used to create a biological network that displays the relation between gene and miRNA expression data.

\subsubsection{Transcriptomic data processing}

Many studies in biomedical science have arisen from experiments showing transcriptomic changes under specific in vitro or in vivo conditions for a particular cell or organism. In most cases, this results in the generation of a list of differentially expressed genes for defined treatments/conditions. With the modernization of sequencing techniques it is possible to generate detailed functional genomics data of an organism model in a fast and cheap manner. The popularity of these techniques has led to the generation of enormous quantities of genomics data in a short period of time, which has brought problems in terms of storage, accessibility and analysis of these data. These issues have created an interesting debate in the science community, leading to the concept of FAIR data [259] which states that data has to be Findable, Accessible, Interoperable and Reusable. Because of FAIR, in publications it is now good practice and, sometimes compulsory, to upload any functional data generated to public repositories and generate a form where a detailed experimental description is provided together with (un)processed data. The most common public repositories for omics data are Gene Expression Omnibus (GEO) [63] and Array Express (AE) [12], on-line portals where functional genomic data and sequencing techniques, such as microarray, RNA-seq and ChIP-seq are findable with key words related to the study. The corresponding web-page of the study provides experimental information such as the type of organism used, description of the generation of each sample, the analytical platform used to assess gene expression and the actual data. Finally, unprocessed (raw) data, obtained from gene expression assays, will be provided in a specific format that will depend on the technique and platform used, therefore this creates an additional issue to the reproducibility of the analysis tools. Functional analysis of transcriptomic datasets requires three important steps: quality control of the data, data normalization and statistical analysis . The first step consists on the analysis of the 1) quality control of the experimental raw data that will indicate if there has been any technical issues that could affect the result, which will lead to the question if the removal of any sample for the analysis is needed; 2) a normalization strategy based on the idea that in a gene expression analysis, overall gene expression is not altered and variations in overall expressions between samples must be removed without affecting the dynamic profile. Thus, a normalization method is applied to equalise the mean intensities of all samples; 3) a statistical approach is carried out to reveal which genes are significantly altered under certain conditions and finally 4) gene annotation step assignates gene IDs to the individual gene expressions measured from a specific gene database. 


\subsubsection{Pathway analysis}

Using available high-throughput genomics data often makes it possible to retrieve a full overview of the changes in gene expression that occur in a particular biological system. Such data are valuable for understanding the molecular changes within cells or tissues. Normally, the initial hypothesis of a study forces the researcher to focus on one specific part of the system, often resulting in the analysis of only a small part of the genes analysed to test the initial hypothesis. However, greater biological insight can be achieved when the effect on all measurable genes is considered and analysed. Thanks to the development of bioinformatics tools and the generation of databases of different biological features, it is now possible to exploit functional genomic data at many levels.

\section{WikiPathways}

In the history of biochemistry and biomedical research, in order to describe molecular reactions and interactions, schematic representations were used to explain the theoretical molecular dynamics that were described in a cell or tissue. In this modern era, the computational adaptation of these diagrams has allowed the science community to represent biological diagrams that represent these dynamics in a standardized, open and reproducible way. In consequence, within the last two decades there has been a great interest in the annotation of biological pathways in databases for research usage; resulting in the creation of online libraries that contain collections of diagrams that represent biological processes which are available for their free use, such as the well known KEGG and Reactome repositories.

In the current project, we have focused on the usage of a specific pathway repository: WikiPathways. WikiPathway is an open, free and user-friendly pathway repository which includes diagrams representing biological pathways from over 25 different species. Since it is an open database, everyone can join the community and create a new biological pathway in the database to be curated and used by other users. The WikiPathways curation team consists of a joint collaboration between different international Universities and institutions having over 730 contributors [222] that weekly curates old and new pathways in order to keep a complete and valid database that contains biological processes from different cell types that are referenced with published studies. To date, in March 2020, there are more than 2800 pathways registered in WikiPathways that, with the community's work, covers over 11500 unique human protein coding genes [222].

Each pathway is formed by nodes representing biomolecules (genes, proteins and metabo- 
lites) connected by edges that represent reactions, mobility or interactions. The striking feature of this format is that each feature of the pathway contains detailed information of the gene, protein or metabolite. The key to these annotations is the link between different related biomolecule IDs provided by the BridgeDb software that allows the connection of the pathway nodes to other databases or experimental datasets [242]. For instance, in most cases, each gene node can be linked to gene identifiers from several databases such as Entrez, Ensembl, Agilent, Ilumina, etc; and metabolite nodes can be linked to well-known databases, e.g ChEBI and HMDB [222]. This link between databases allows the user to analyse omics experimental data at the pathway level with the objective to perform a statistical pathway analysis or plot and visualize the data in the pathway diagrams.

\section{PathVisio}

PathVisio [136] is a user-friendly bioinformatics software tool that contains several features that make it possible to have a better understanding of biological mechanisms. This program is capable of performing a pathway analysis with three main functions: pathway editing, omic data visualization, and pathway statistics analysis. Combining all this together, PathVisio has the potential to study and compare experimental datasets at the pathway level, highlighting potential biological processes that might be altered under certain conditions [136]. Pathway creation and annotation are crucial to portray biological processes in diagrams that represent the dynamics of biomolecules. PathVisio's extensive editing features allows the user to create a completely new pathway using graphical objects to represent the biological components. The BridgeDb database annotation included in PathVisio, enables the cross annotation of each node-component, like genes/proteins and metabolites of the pathway allowing the link between the pathway components and external omics databases. With the WikiPathways plugin for PathVisio a created pathway can be uploaded to the WikiPathway repository where it will be curated by the community promoting the growth of the platform by professional researchers from different fields. Furthermore, with the mentioned plug-in, the user can also locally download every pathway stored in the repository for editing and data-visualization.

PathVisio also provides the option to visualize experimental data in the pathway, data is linked from each biomolecule measured to every node in the pathway. Providing logical rules based on data parameters from the dataset provided, it is possible to colour the nodes showing how, for example, the expression of a gene changes based on the experimental conditions. Furthermore, different types of omics data, such as transcriptomic, metabolomic or proteomic, can be incorporated in the same analysis in PathVisio being able to provide a more detailed analysis of a system. Finally, as the study that we have performed in Chapter 
4 shows, it is possible to compare different dataset of the same nature but from different organism or cell models using this software [136]. The last major feature of PathVisio is the performance of a statistical pathway analysis that will highlight the most significant pathways altered considering the experimental dataset provided. This strategy aims to consider an entire omics data and contextualize it at the pathway level based on a logical statistical rule. PathVisio performs an over-representation analysis which is evaluated by $\mathrm{Z}$ score calculated by a statistical test that follows a hypergeometric distribution. This statistical approach takes into account the components that are annotated in the pathways, all the biomolecule-identifiers from dataset that are present in at least one pathway and the ones that satisfies the criteria established in the logical rule [136]. The output of this analysis is a ranked list of pathways that are significantly altered based on an experimental dataset.

\subsubsection{Integrative network analysis}

Biomedical science is living an era where the capacity of generation and analysis of data has become one of the priorities for research. The generation of large scale datasets and the continuous databases growing faces us with the problem of having to connect different aspects of molecular biology to understand how a whole system works. One solution is the representation of such associations by the generation of biological networks that represent molecular dynamics or interactions. Here we will describe the software tools and databases that we have used to exploit public dataset that have studied the role of vitamin D in different immune cell models previously analysed at the pathway level.

\section{Cytoscape}

Cytoscape is an open-source network analysis software tool that enables the analysis of large scale datasets that can be used to study biomolecular interactions from functional experimental data. The main feature of Cytoscape is to analyse and visualize data as a network perspective easing the identification potential interactions within the biological components included in the datasets (https://cytoscape.org/). Cytoscape can integrate data from real experimental datasets generating nodes connected by edges representing the interactions between biomolecules. The generation of a biological network is accompanied by the integration of attributes to the nodes and edges using annotations from either parameters of the dataset used or information of molecular aspects from external databases. These annotations in combination with the use of dedicated Cytoscape apps allows the selection of the network components for selective manipulation, gene expression changes visualization and generation of subnetworks for further analysis. [219]. 
In the first part of our analysis, we were able to identify a subset of genes that were commonly regulated by vitamin D in different immune cell models. Different apps are available for Cytoscape that exploits data at many molecular biology levels. For instance, it is possible to represent the WikiPathways diagrams in Cytoscape either as a pathway or in network format for data visualization and analysis [134]. Gene ontology analysis using the ClueGO app can be performed to identify enriched biological terms in a defined cluster of genes, and the app can show the overrepresented gene ontology terms and pathways [164]. Furthermore, it is possible to extend these results by the use of different databases to identify and study validated gene-molecule interactions such as with miRNAs using the CyTargetLinker app [133] or protein-protein interactions using String app [61]. Finally, as we described before for PathVisio, Cytoscape offers the possibility to incorporate experimental parameters in the network and to customize the network layout to represent the experimental results based on for instance color rules.

\section{Reporter metabolite analysis: RAVEN tool}

In addition to the development of pathway analysis tools to better interpret changes in mRNA and protein expression on a genome/proteome-wide basis, the creation of large databases of metabolites, enzymes and their reactions has provided new tools to better understand the complexity of the cell metabolic status of the cell. In this $\mathrm{PhD}$ project, the initial observation of $1,25(\mathrm{OH})_{2} \mathrm{D} 3$-induced changes in DC metabolism based on pathway analysis of genomic data, prompted us to use metabolomic bioinformatic tools to further investigate the transcriptomic datasets and better understand the metabolic status of the different immune cells based in the presence of vitamin D. Gene expression datasets can be contextualized in a metabolism system using the RAVEN toolbox that uses a metabolomic map where enzymes, metabolites and reactions are documented in a database. The RAVEN toolbox is a Matlab based software that, among its utilities, is capable of taking into account gene expression data into a metabolic map, implemented in a database. This feature can be used to highlight metabolic aspects of vitamin D in the immune system. Thanks to the implementation of the RAVEN bioinformatic software, it is possible to report potential metabolites, considered hot points of regulation, using gene expression datasets [253]. The results of the reporter metabolite analysis, revealed interesting aspects of the role of $1,25(\mathrm{OH})_{2} \mathrm{D} 3$ in DC cell metabolism and provided the foundation for the second part of the project and helped with the wet-lab experiment design and decision making. Finally, the interpretation of all the bioinformatic results from available data was the preparatory step to the wet-lab approaches 
and, in the same way, bioinformatic tools were used to help to interpret the results of the wet-lab experiments; keeping in contact with the two fields of science.

\subsection{Objectives. A return journey: from dry to wet lab}

The overarching aim of this $\mathrm{PhD}$ project was to connect wet and dry laboratories. We used bioinformatic tools to explore the effects of vitamin D on global gene expression and biological processes in immune cells, and wet laboratory experiments to validate these effects and to explore them in more depth (figure 1.6). Most conventional biomedical research studies consist of the following sequential events: formulation of a hypothesis, experiment design, laboratory approach, analysis and discussion of results. Here we decided to start our project with an additional first step: analysis and exploitation of available transcriptomics data that is related to the central hypothesis.

Bioinformatic analysis of publicly-available gene expression datasets enables identification of previously unrecognised biological responses to vitamin $D$ of immune cells.

To test this hypothesis the $\mathrm{PhD}$ project commenced at Maastricht University to define the types of bioinformatic workflows that could be used to explore vitamin D function in specific target cells (see Chapter 2). The second step, also carried out at Maastricht University, was to select available gene expression datasets that could be applied to the bioinformatic workflows designed for in-depth analysis of vitamin D function. Here we chose to analyse myeloid immune cells because of the relative abundance of gene expression datasets available for related myeloid cell types (monocytes, macrophages and DC). Selection of datasets was restricted to studies that assessed the myeloid cell impact of the active form of vitamin $\mathrm{D}, 1,25(\mathrm{OH})_{2} \mathrm{D} 3$ (see Chapter 4). The third phase of the $\mathrm{PhD}$ was to apply information and strategies developed in Chapter 2 to ongoing studies of vitamin D within teams at the University of Birmingham. Here the objective was to use new bioinformatic strategies to improve interpretation of experimentally-generated datasets from non-classical (decidua) vitamin D target tissues (see Chapter 3). The final stage of the $\mathrm{PhD}$ at the University of Birmingham was to carry out experimental laboratory work based on information derived from studies in Chapters 2-4. In this case, studies of primary cultures of human DC were carried out to explore in greater detail the bioinformatic identification of $1,25(\mathrm{OH})_{2} \mathrm{D} 3$-induced changes in cell metabolism as a major feature of immune responses to vitamin D (see Chapter 5). 
The overall conclusion from studies is that comprehensive, statistically defined, analysis of transcriptomic datasets in a 'dry lab' environment enables identification of key regulatory pathways for vitamin D for more refined and targeted analysis in 'wet lab' experiments. This approach streamlined experimental design and greatly accelerated novel findings from experimental studies. In addition, the bioinformatic approaches identified in this $\mathrm{PhD}$ provided an improved system for assessing the impact of vitamin D on different tissues and cells. Our experimental lab research focused on the effects of $1,25(\mathrm{OH})_{2} \mathrm{D} 3$ on immunometabolism in several types of DC, highlighting the ability of this hormone to promote tolerogenic DC development. This, together with initial findings from the analysis of public gene expression datasets in the first stage of this $\mathrm{PhD}$, led to our novel finding that $1,25(\mathrm{OH})_{2} \mathrm{D} 3$ is a key regulator of fatty acid synthesis in DC.

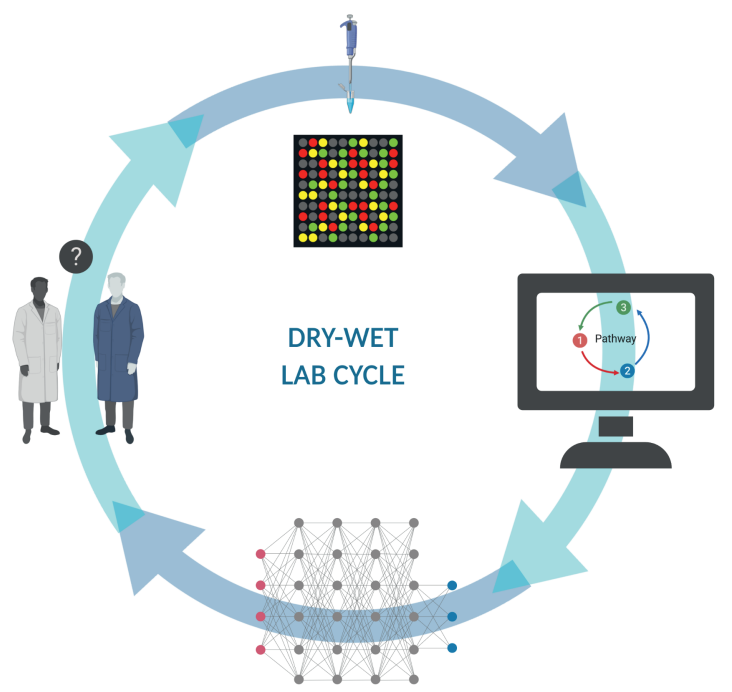

Fig. 1.6 A role for bioinformatics in the interpretation and design of experimental research. The two fields of wet and dry lab are in constant contact in biomedical research leading to a cycle work routine in which experiment can be designed based on previous dry-lab analysis and the results of these experiments can be analysed with the same tools. 


\section{Chapter 2}

\section{A bioinformatics workflow to decipher transcriptomic data from vitamin $D$ studies}

Published in The Journal of Steroid Biochemistry and Molecular Biology in 2019, DOI: 10.1016/j.jsbmb.2019.01.003

Amadeo Muñoz García ${ }^{1,2}$, Lars M. Eijssen ${ }^{1}$, Martina Kutmon ${ }^{1,3}$, Chaitra Sarathy ${ }^{3}$, Ardi Cengo $^{1}$, Martin Hewison ${ }^{2}$, Chris T. Evelo ${ }^{1,3}$, Michael Lenz ${ }^{3}$, Susan L. Coort ${ }^{1}$

\footnotetext{
${ }^{1}$ Department of Bioinformatics - BiGCaT, NUTRIM School of Nutrition and Translational Research in Metabolism, P.O. Box 616, Maastricht University, Maastricht, the Netherlands ${ }^{2}$ Institute of Metabolism and Systems Research, The University of Birmingham, Birmingham, B15 2TT, United Kingdom

${ }^{3}$ Maastricht Centre for System Biology (MaCSBio), Maastricht University, Maastricht, the Netherlands
} 


\subsection{Abstract}

The first part of this $\mathrm{PhD}$ project was aimed at identifying workflows that connect experimental laboratory studies and bioinformatic research to better understand genomic datasets on multiple levels. Open access data sources and the availability of numerous bioinformatics tools has enabled the creation of various pipelines capable of analysing complex biological models, but the lack of connectivity between bioinformatic workflows complicates their effectiveness. Here, we present a detailed description of a workflow composed of different bioinformatics tools that exploits data from large-scale gene expression experiments, contextualizing them at many biological levels. To illustrate the relevance of our workflow for the vitamin D community we applied it to data from myeloid cell models treated with the hormonally active form of vitamin D. From raw files of functional genomic studies it is possible to utilize complete transcriptomic information to obtain a better biological insight into the model being studied. Different software and algorithms are included to analyse at pathway, metabolic, ontology and molecular biology level the effects of altered gene expression. Furthermore, the use of different databases to analyse gene expression data allows us to perform a complete interpretation of functional genomic studies, and the implementation of analysis and visualization software tools enabled better interpretation of the results. This review is an example of how to select and bring together several software modules to create one pipeline that processes and analyses genomic data at several biological levels making it open, reproducible and user friendly. The primary outcome of the integrated multifaceted bioinformatic pipeline was the identification of a novel metabolic function for vitamin $\mathrm{D}$ in antigen-presenting dendritic cells. However, as part of the wider $\mathrm{PhD}$ project, this strategy was also applied to other biological systems to enable an unbiased and novel analysis of the effects of vitamin $\mathrm{D}$ in different cells models.

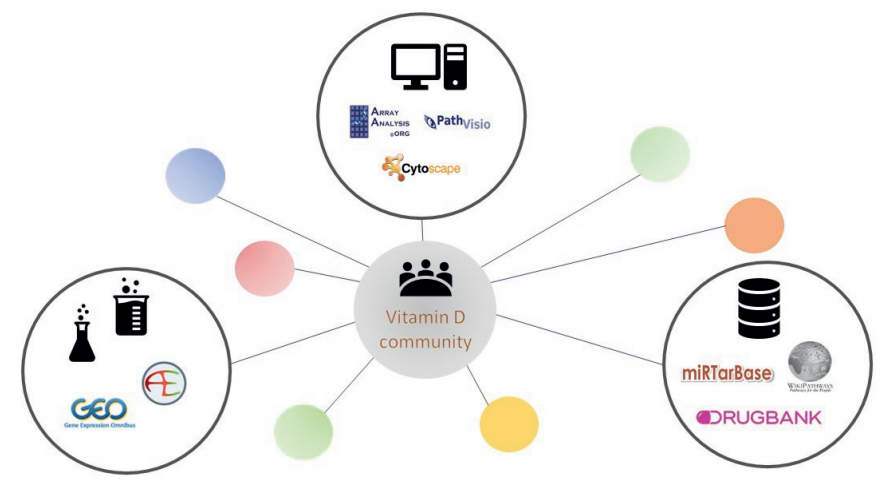




\subsection{Introduction}

The wealth of data produced in the last decades as a result of functional genomic studies has greatly contributed to our understanding of how nutritional factors affect biological processes. In the field of vitamin D research, many genomic studies have investigated effects of the active hormonal form of vitamin $\mathrm{D}\left(1,25\right.$ dihydroxyvitamin $\left.\mathrm{D} 3\left(1,25(\mathrm{OH})_{2} \mathrm{D} 3\right)\right)$ at gene and/or protein level in various cell types $[231,27,70]$. However, an in depth analysis of the functional consequences of large-scale molecular changes is often lacking. In the current paper we showcase a straightforward and available bioinformatic workflow to perform an advanced, flexible and integrative analysis of high-throughput transcriptomic data.

\subsubsection{Transcriptomic data}

To store, share and re-use the generated high-throughput data, online available storage databases, i.e. gene expression omnibus (GEO [50]) and ArrayExpress [182], were developed. Initially, these databases captured transcriptomic data from microarray studies, but nowadays they contain a variety of molecular data including sequencing data. Also vitamin D related large-scale expression studies are available online and contain data from different cell types and tissues in various species. Together with the continuous growth of transcriptomic data, comes the difficulty of processing and interpreting it at functional level. Most of the expression studies are lacking dry lab applications that make it possible to exploit the entirety of the experimental data. Simultaneously with data generation an increasing number of informatics tools have been created to allow data analysis, resulting in the bioscience field known as bioinformatics. The main aim of this development is to process, interpret and integrate data in a more efficient and reproducible way. One of the biggest obstacles to make this possible and to create a better link between experiment data generation and analysis is the lack of a documentation that explains to researchers from different science backgrounds how to use various tools for a main purpose to decipher data for biological interpretation.

\subsubsection{Bioinformatics tools}

The application of bioinformatics tools in bioscience research helps to interpret data and will generate research questions that mark the starting point of experimental validation studies. An additional advantage of the implementation of open bioinformatics tools is the possibility to analyze public genomic datasets from repositories enabling the screening of previous studies with a straightforward protocol. In the current paper the application of a straightforward bioinformatics workflow to (re-)analyze transcriptomic data will be 
explained in relation to vitamin D studies. In our previous paper [81] we implemented and used a bioinformatics pipeline resulting in new insights into the role of $1,25(\mathrm{OH})_{2} \mathrm{D} 3$ in immunometabolism processes in the innate immune system. Here, the applied tools and methods will be demonstrated and extended to provide a complete overview of analysis steps needed for functional data analysis.

\subsubsection{Data analysis workflow}

The guide of our data analysis pipeline includes several bioinformatics tools grouped in a workflow that exploits transcriptomic data and positions them into a functional biological context. Once the datasets of interest have been selected, the first step consists of the application of open and free analysis modules in ArrayAnalysis [64] to check the quality, normalize, and process raw transcriptomic data obtaining the parameters that show the gene expression changes. Second, the processed dataset is used in PathVisio [136], an open and freely available biological pathway editor and analysis tool, to perform a statistical analysis that highlights the biological processes that are significantly altered. Moreover, in the pathway diagrams, using the pathway repository of WikiPathways [221], which includes all types of biological processes, the changes in gene expression are visualized in a comprehensive manner. In addition, newly created vitamin D-related pathways, using the program PathVisio, were added to the human pathway collection of WikiPathways. Third, to study metabolic processes affected based on gene expression data, the RAVEN toolbox is used to identify crucial regulation points of a metabolic model [7]. Fourth, to retrieve more information about an affected pathway, existing knowledge on factors that are related to the pathway genes are added to the pathway using the network analysis tool, Cytoscape [223] and Cytoscape apps, i.e., WikiPathways app [134] and CyTargetLinker app [133]. Finally, the overrepresented gene ontology classes in a selected subset of genes are visualized in a network using the Cytoscape app, ClueGO [22]. 


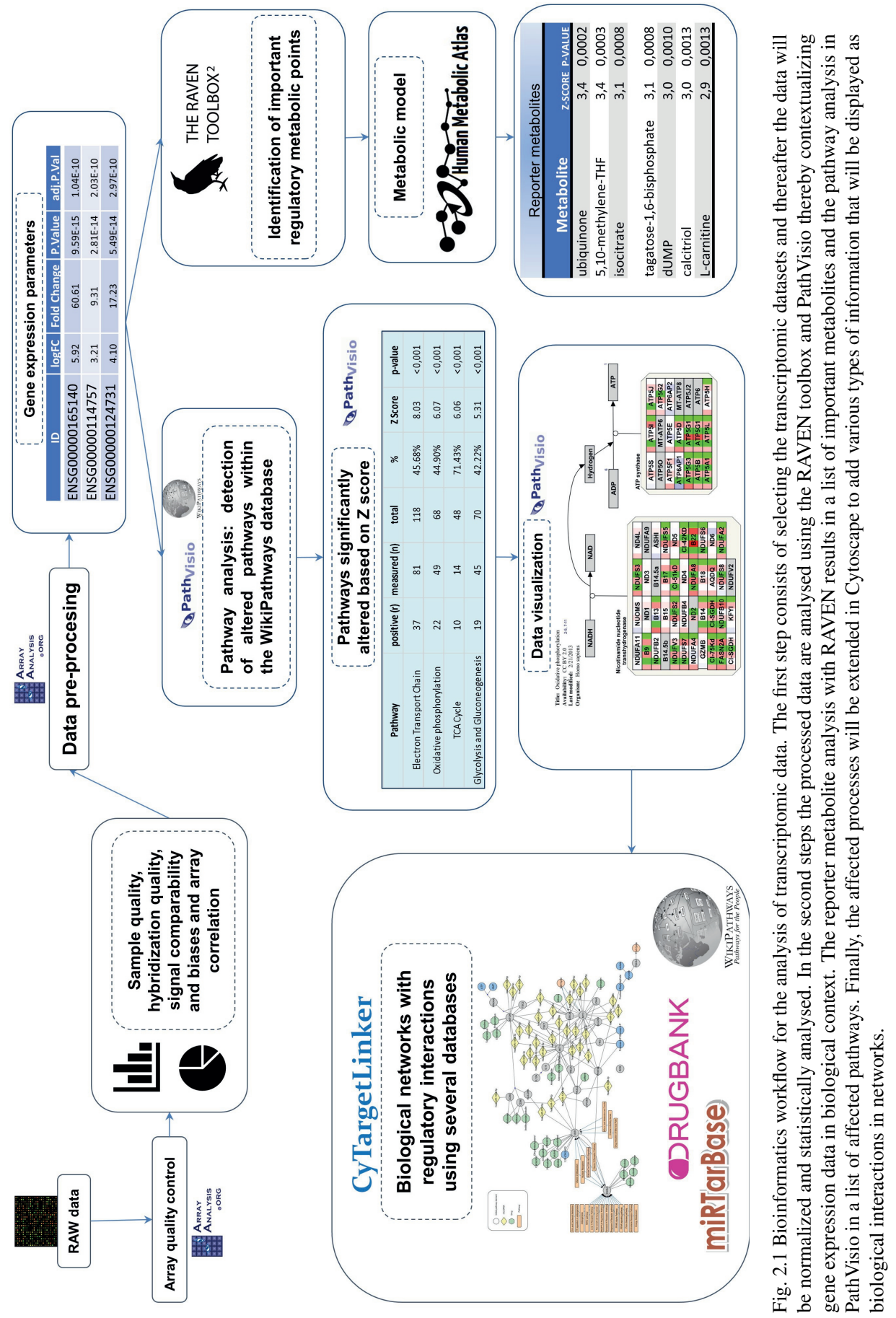




\subsection{Materials and methods}

\subsubsection{Retrieving gene expression data from public repositories}

Open sources promote the generation of platforms where data is curated and shared by the scientific community to encourage access, usage and promote experimental reproducibility. In the recent years, an increasing number of publications related to gene expression studies are accompanied by a datasheet archived in a data repository that contains detailed information about the experiment and the raw and/or processed gene expression datasets. The two most known repositories are ArrayExpress (https://www.ebi.ac.uk/arrayexpress/, [121]) and Gene Expression Omnibus (GEO, http://ncbi.nlm.nih.gov/geo/, [63]). It is possible to explore these repositories to find genomic data by specifying keywords that are related to the biological question of interest such as organism, cell type, gene, molecule, type experiment, etc. Detailed information is available on each dataset from ArrayExpress and GEO containing value information of the experiment and, if it is available, the publication related to it. The experimental page gives an overview of the laboratory protocol design which includes a detailed explanation of each samples characteristics, making it possible to keep track of the data for each sample in further analyses.

\subsubsection{Sharing large-scale expression studies}

In every case, the result of microarray analysis is uploaded to make it publically available to the scientific community. This kind of format depends on the platform used in the experimental design and different approaches are needed depending on the format of this data. In the vast majority of the cases, the raw data is available which promotes the analysis of its quality and the possibility to detect anomalies in arrays and remove them in further steps. Alternatively, authors also submit normalized gene expression data provided with information detailed of which methodology was used to process it.

\subsubsection{Selection vitamin D-related transcriptomic studies in immune cells}

The comparison of different datasets requires selection criteria that will allow that any conclusion obtained of this analysis will have a logical interpretation. The criteria followed to select datasets of myeloid cells treated with vitamin $\mathrm{D}$ was based on the experiment design that was set to study the effects of vitamin D at gene expression level. First, the cell model has to be related to the myeloid family, more specifically, cells derived from monocytes. 
Secondly, in all cases cells were treated with $10-100 \mathrm{nM}$ of the active form of vitamin $\mathrm{D}\left(1,25(\mathrm{OH})_{2} \mathrm{D} 3\right)$ and all datasets that used analogs or other forms of the hormone were excluded. Moreover, the duration of the treatment was restricted to a minimum of $24 \mathrm{~h}$ and a maximum of 5 days of treatment. Finally, only studies in human cells that used microarray technology were selected.

\subsubsection{Processing raw gene expression data: ArrayAnalysis}

In transcriptomic data analysis, it is assumed that most of the genes measured will not be changed in the different conditions. Differences in gene expression can be caused by the experimental conditions, but also the methodology followed can provoke unwanted variations that must be removed. The department of bioinformatics of the Maastricht University (Bioinformatics-BiGCaT) has developed a workflow that can process raw gene expression data applying a quality control (QC) module, statistical analysis and pathway analysis to gene expression data: http://arrayanalysis.org/ [64].

\subsubsection{ArrayAnalysis modules}

The first module of array QC consists in uploading raw gene expression data with simple annotation that specifies the group of each array. This first approach analyses the unprocessed data and will create a report that includes quality control plots that are grouped in categories including: sample quality, hybridization quality, signal comparability and biases and array correlation. All this together will be included in a QC report that will show the quality of the array intensities contributing to the detection of faulty arrays that can be removed from further steps in the analysis. Secondly, the pre-processing module of ArrayAnalysis performs a background correction and intensity normalization. Several normalization methods are available, including RMA, gcRMA, MAS5 and Plier. In this second module, the re-annotation of the data is also included, making it possible to assign probes with alternative gene identification databases (i.e Ensembl, ENTREZ, UniGene, etc). Finally, an evaluation of the whole process is performed showing the distribution of the intensities in different plots after the pre-processing of the dataset that are included in a report of the whole process of module one. The resulting pre-processed data consist in corrected and normalized raw data that can be the object of statistical analyses that aim to compare gene expression data of different sample groups. The statistical approach will produce a table with each gene ID with several gene expression parameters that indicate if its expression is significantly altered or not based on certain conditions. The second module of ArrayAnalysis can perform this statistical approach. First, a group specification on each array is needed to 
make a comparison of gene expression changes between different conditions. Taking into account the experimental details from the main page of the dataset it is possible to know the nature of each sample allowing the correct grouping in this second module of ArrayAnalysis. Each array has a source name that has to be followed by "factor value" that corresponds to the group sample to which it belongs. Groups that are going to be compared are set, and then the statistical approach can be performed. The resulting output consists of statistical parameters of each gene ID measured and compared. This kind of format data can be used by different bioinformatic tools to extract biological interpretation out of it.

\subsubsection{Analysis of selected vitamin D-related transcriptomics datasets}

A total of 6 datasets were selected by Muñoz García et al. [81] to perform a analysis of gene expression datasets of myeloid cells treated with the active form of vitamin D but to make comparison of the three different cell types, three datasets were highlighted for THP-1 cells (GSE52819, [248]), dendritic cells (GSE13762 [231]) and monocytes (GSE56490 [72]). The quality control of arrayanalysis.org, performed by an R script containing several functions from Bioconductor, revealed that there was no need to exclude any samples. Different methods were suggested by ArrayAnalysis to normalize the datasets: Robust Multiarray Average (RMA) for THP-1 and dendritic cells datasets and GeneChip-RMA for monocytes datasets. Then, a statistical analysis was performed using functions from the R package of limma [206] to obtain the statistical parameters that indicate the significance of the gene expression changes.

\subsubsection{Creation of vitamin D related processes}

PathVisio allows the user to create and edit diagrams that shows biological pathways made of nodes and lines that represent biomolecules and their interactions, respectively. The components of the pathways can be annotated with an identifier that is present in the various BridgeDb mapping databases. Currently, these databases are available for i) genes and gene products, ii) metabolites, iii) gene variants and iv) biochemical reactions and interactions. The correct annotation of each pathway element, the upload to the repository of WikiPathways and the curation process allows the users to combine the pathway content with "omics" data. Researchers often use pathway diagrams in their articles to explain in a schematic way how biomolecules interact in organism of study. It is possible to use these schematics diagrams to create new pathways in PathVisio and tag them with key words that are related to the study of origin. Including this annotated diagrams related to vitamin D in this open encyclopedia of pathways makes possible its curation by the scientific community promoting 
and validating its usage. WikiPathways, to this date, has over 400 pathways related to vitamin $\mathrm{D}$ in all species. In this list, different types of pathways are included: cascade signaling, gene expression modulation, metabolic process, etc. Thanks to the work of the WikiPathways community, it is possible to put gene expression context in these pathways making a tool that is reproducible, userfriendly and efficient to identify altered vitamin D processes using experimental transcriptomic data.

\subsubsection{Pathway analysis: visualization and statistics}

Context matters to understand gene expression variations. Largescale transcriptomic analysis can generate many gigabytes of data that are challenging to process in terms of both time and effort. Here we propose PathVisio and WikiPathways as tools to perform a pathway analysis on the gene expression datasets selected to highlight biological processes that are altered. PathVisio is an open software use for pathway visualization and analysis, and WikiPathways is an open platform that stores over 2300 biological pathways of 25 different species for data visualization and analysis [221].

\section{Visualization of omics data on pathways}

In the PathVisio program it is possible to visualize biological diagrams from the WikiPathways repository and display gene expression data parameters, generated by the statistical analysis module of ArrayAnalysis, on the diagrams showing how the gene expression is affected. The elements in the pathway diagram that represent biomolecules, (i.e. genes, gene products and metabolites,) contain general information about the biomolecule. The BridgeDb identifier mapping databases in PathVisio [242] can be used to link transcriptomic, proteomic and metabolomic data thereby recognizing the identifiers from data uploaded allowing inclusion of expression parameters to pathway element. The visualization in PathVisio has two options, either one of the predefined color gradients or setting a rule linked to one color. These options enable the coloring of pathway elements based on the experimental data creating a general view of how the biomolecules are modulated in an experiment designed based on the obtained data. The current study used the most common application in which transcriptomic data is displayed on a selected pathway shows how genes are down-regulated or up-regulated based on the logarithm of the fold change value (log2FC) and the significance of that change based on the $\mathrm{p}$ value. 

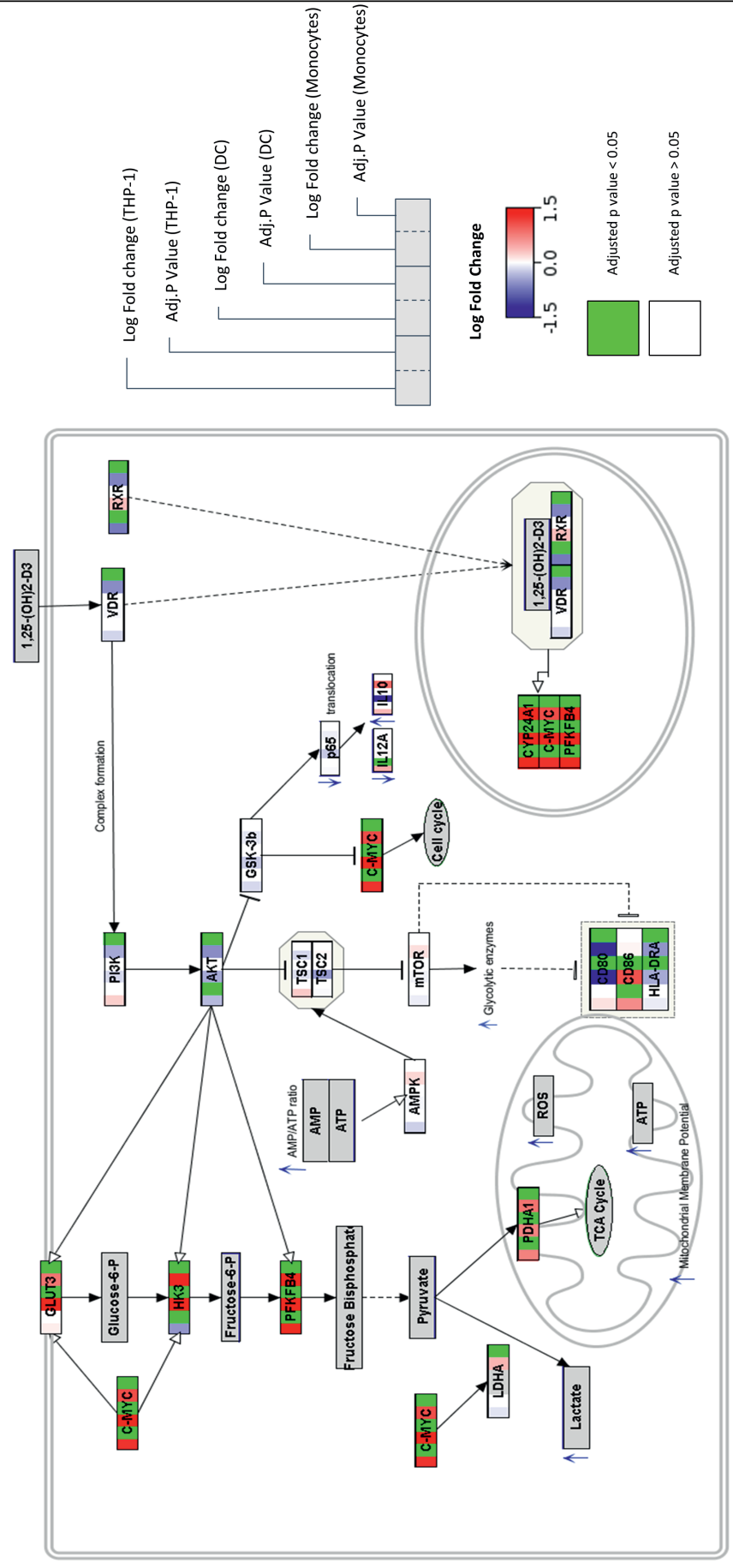

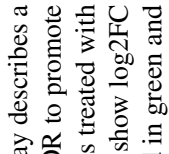

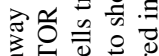

焉焉总

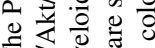

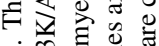

高离声

$\sum \stackrel{0}{ \pm} .0$

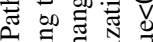

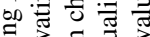

길

N

늘

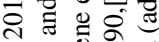

तं .0

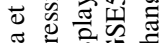

훙.

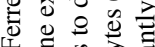

ฐ

漹

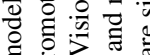

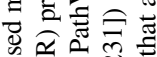

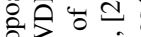

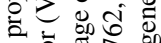

\& 율

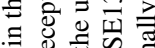

氙氖江

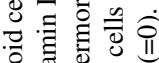

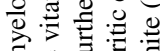

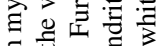

.

品苛全

츨을

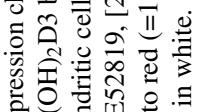

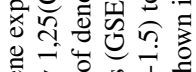

可

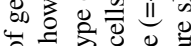

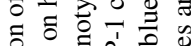

.을 仓元

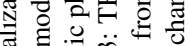

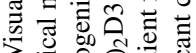

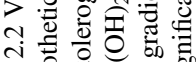

a 


\section{Over-representation analysis}

PathVisio integrates a workflow that makes it possible to upload processed expression data to perform statistical analyses at the pathway level and to contextualize the expression changes at the functional level, highlighting those biological processes that are significantly affected. First of all, a pathway repository is needed to perform the pathway analysis. Currently two major pathway databases provide a collection of pathways compatible to PathVisio, i.e., WikiPathways and Reactome [68]. In our example, processed gene expression data of myeloid cells treated with the active form of vitamin D, 1,25-dihydroxyvitamin D3 $\left(1,25(\mathrm{OH})_{2} \mathrm{D} 3\right)$ was investigated with pathway analysis to find which biological processes are mainly affected by $1,25(\mathrm{OH})_{2} \mathrm{D} 3$ in the different myeloid cells types. Once both gene and pathways databases and the gene expression dataset are loaded in PathVisio, all the needed elements are present to perform the overrepresentation analysis. First, a criterion has to be defined to select which genes are going to be considered as differentially expressed. In most cases, statistically analysed gene expression data generates for each gene a series of parameters that indicate how significantly its expression is altered under certain conditions. Based on these parameters PathVisio perform an overrepresentation analysis highlighting those biological processes that are significantly altered within a pathway repository. For each pathway a Z-score is calculated by a standard statistical test under the hypergeometric distribution, as previously described [136]. The resulting output gives a list of pathways that are ranked based on Z-score. Pathways with a Z-score above the cutoff value of 1.96 will be considered as affected biological processes since it indicates if the pathway shows a difference in the ratio of differentially expressed genes as compared to the complete gene list.

\subsubsection{Reporter metabolite analysis}

The effect of external factors on cells in cultures can be studied by analysing the metabolic changes that occur within the cell. A major advantage of functional studies such as microarray assays and RNA-seq is that they reveal how certain factors can modulate gene expression and, as described above, these data can be put in a biological context and used to determine how these factors influence biological processes; including in most cases metabolic processes. Thanks to pathway analysis and visualization options of PathVisio, it is possible to see the key genes mainly affected from the ranked list of metabolic pathways. Studying the metabolic responses to cells to certain factors, has to be included in the strategy since identification of concentration changes of metabolites gives value information to study how the cell responds to specific conditions. It is possible to go further with the analysis of gene expression analysis and contextualize this kind of data at the metabolite level. Kiran Raosaheb Patil 
and Jens Nielsen have developed an algorithm capable of identifying so-called reporter metabolites within a genomescale metabolic model, where a map of reactions is represented by enzymes and metabolites [183]. The algorithm is implemented in a bioinformatics matlab tool called RAVEN (Reconstruction, Analysis and Visualization of Metabolic Networks) [https://github.com/SysBioChalmers/RAVEN] which identifies key points in a genomescale metabolic model using gene expression data. First, the bioinformatics tool will take into account gene expression data to score the enzymes based on how significantly changed their gene expression is under the experimental conditions. Then metabolites of these processes will be scored based on the scoring of the neighboring enzymes. This analysis will identify potential metabolites that can be considered as hot points of metabolic regulation under the conditions studied in the datasets selected.

\subsubsection{Extension of biological pathway diagrams from WikiPathways in cytoscape}

Pathways from WikiPathways harbor information on genes, proteins, metabolites and interaction types. To retrieve additional information regarding the genes and proteins, the pathway was extended with various types and levels of biological knowledge. The extension of the pathway was performed in the open source and freely available network analysis tool, Cytoscape [223]. First, the selected pathway from WikiPathways was uploaded into Cytoscape as a network using the WikiPathways app [134]. Second, linksets containing information about interactions between gene-pathway from WikiPathways, drug-targets from DrugBank [261] and experimentally validated microRNA-targets from miRTarBase [44] were downloaded (https://projects.bigcat.unimaas.nl/cytargetlinker/linksets/). Third, the network was extended with drug-target, microRNA-target and pathway-gene interactions with the CyTargetLinker app [133]. Fourth, by applying filtering options in Cytoscape the following parts were selected; i) Approved Drugs from DrugBank, ii) Experimentally validated microRNAs with functional microRNA-target interactions (MTIs) and iii) Curated human pathways in which the nuclear receptors RXRA and the nuclear vitamin D receptor (VDR) are present. As a result a newly created network contains the genes, proteins, metabolites, pathways and interactions from the selected process and the added interactions of the selected and filtered LinkSets. 


\subsection{Results and discussion}

\subsubsection{Workflow}

The pipeline proposed brings together several open bioinformatics tools to process transcriptomic data to highlight biological processes (figure 2.1). The first step consists of data retrieval from public databases such as GEO and ArrayExpress. Secondly, selected raw data is processed by an $\mathrm{R}$ based module implemented in ArrayAnalysis to normalize and produce the statistical parameters. Thereafter, the processed data is used by several bioinformatics tools. First, PathVisio processes the data to highlight which biological pathways are significantly altered. Second, in PathVisio the analysed data is visualized in the actual pathways. Third The RAVEN toolbox has as input also the statistically analysed data and helps to identify which metabolites are more likely to be subject to regulation. Finally, it is possible to extend pathways using Cytoscape to create networks that link pathway, gene, protein, metabolite, microRNA and drug information.

\subsubsection{Pathway analysis using public datasets: vitamin $D$ and gene ex- pression modulation in myeloid cells}

The process and analysis of publicly available transcriptomic datasets allowed Muñoz García et al. [81] to decipher the biological processes altered by $1,25(\mathrm{OH})_{2} \mathrm{D} 3$ in different types of human myeloid cells. To do that, a total of six datasets were included in this article containing raw microarray data from gene expression modulation by $1,25(\mathrm{OH})_{2} \mathrm{D} 3$ in THP-1 cells, dendritic cells (DC) and monocytes. In all three kinds of myeloid cells, $1,25(\mathrm{OH})_{2} \mathrm{D} 3$ treatment promoted the upregulation of genes involved in metabolic pathways: glycolysis, electron transport chain, TCA cycle and oxidative phosphorylation. In addition, the analysis revealed that only in THP-1 cells, $1,25(\mathrm{OH})_{2} \mathrm{D} 3$ silenced the expression of genes involved in cell cycle (table 2.1).

\subsubsection{Pathway editing and data visualization: from references to dy- namic pathways}

The impact of vitamin D in many area of human health, including cancer research, metabolic diseases, bone disease and autoimmune diseases is now recognized. In all areas, the understanding of the molecular action of the different forms of vitamin $\mathrm{D}$ has been always been a priority in most of these studies. All finding related to describe vitamin D molecular signaling are accompanied with a schematic diagrams that explains how biomolecules 
interact in certain pathways. The compilation of these diagrams and their adaptation to WikiPathways is making it possible to compare multiple transcriptomic studies related to vitamin $\mathrm{D}$ and put them in specific biological context. One of the latest studies done in vitamin $\mathrm{D}$ and its implication in metabolic reprogramming in tolerogenic dendritic cells [72], described that PI3K/Akt/mTor signaling pathway was crucial for the development of the tolerogenic phenotype in DC. The adaptation of this pathway in PathVisio and using similar transcriptomic datasets from myeloid cells treated with vitamin D showed that this process is also being up-regulated under vitamin D treatment in similar cell lines (figure 2.2). Another study by Hii and Ferrante [102] summarizes the nongenomic actions of vitamin $\mathrm{D}$ in a comprehensive diagram. It shows the effect of $1,25(\mathrm{OH})_{2} \mathrm{D} 3$ beyond the activation of VDR. The created model of the non-genomic actions is now available at WikiPathways (https://www.wikipathways.org/index.php/Pathway:WP4341). Finally, VDR is identified as a nuclear receptor and upon binding of $1,25(\mathrm{OH})_{2} \mathrm{D} 3$ it becomes activated and regulates transcription of VDR target genes. Binding of VDR to the genomic location occurs as a heterodimer with the retinoic $\mathrm{X}$ receptor (RXR). The interplay between VDR and RXR is also captured in a newly created pathway at WikiPathways (https://www.wikipathways.org/index.php/Pathway:WP4342). These three pathways are now added to the human WikiPathways collection and are part of the curated pathway collection available for download. 


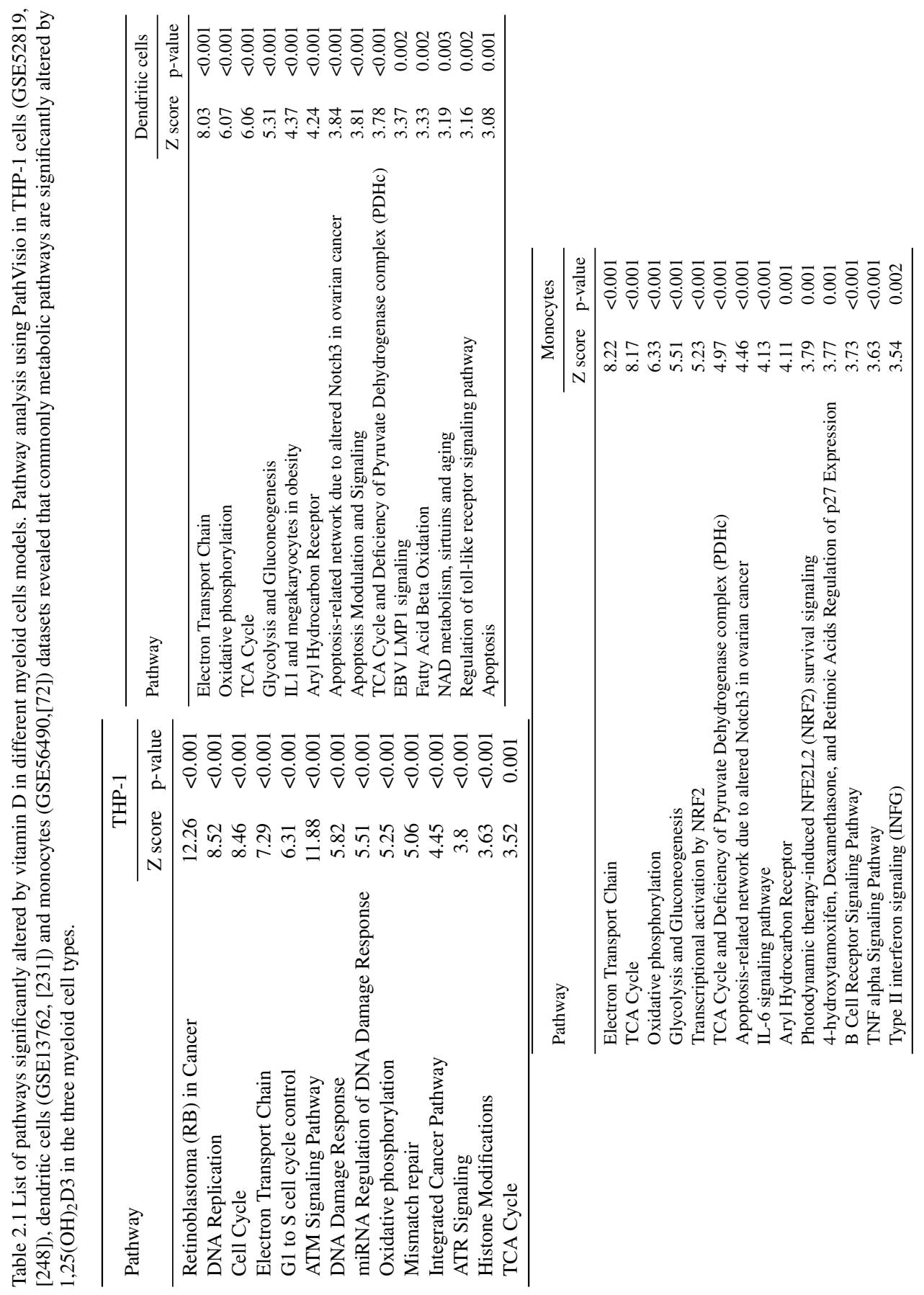




\subsubsection{Integrating multiple datasets in Path Visio}

One of the greatest challenges of functional analysis projects is to compare different experiments concerning the same issue. Sometimes, in one specific experiment all conditions are not considered, but it is possible to find in the repositories mentioned, other similar studies regarding the same scientific question. A spreadsheet table containing the IDs with the gene expression parameters from the different dataset considered that represent different experiments can be uploaded in PathVisio to visualize this kind of comparison. Once PathVisio recognizes the IDs with the correspondent parameters for each condition, it is possible to set the visualization options to compare the gene expression data in the gene nodes of the pathway diagram. Again a set of rules are needed to assign colours to gene expression parameters from each dataset. The resulting diagram will show nodes divided in different sections according to the number of dataset considered. In the example shown in this diagram three datasets from different immune cell types are compared in one pathway showing the changes in gene expression and its significance (figure 2.2). With this strategy it is possible to show similarities and differences between related experiments, patterns in gene expression and identify genes of interest for further study.

\subsubsection{Reporter metabolite analysis}

The usage of The Raven Toolbox allows to analyse transcriptomic data from myeloid cells treated with vitamin D in a metabolic model such as the Human Metabolic Atlas to highlight those metabolites that are crucial in the metabolism regulation based on the gene expression datasets considered. The output of this tool is a list of metabolites that are scored with a $\mathrm{Z}$ score. A high score of a specific metabolite is an indication that the expression of enzymes implicated in its metabolism is being significantly altered by vitamin D (table 2.2). Reporter metabolite analysis of myeloid treated with vitamin D datasets highlighted metabolites implicated in TCA cycle, ETC and OXPHOS pathways: NAD+, $\mathrm{NADH}$, ubiquinol, ubiquinone, fumarate, succinate. Interestingly, metabolites implicated in vitamin D metabolism are also being reported as hotspots of metabolism regulation in these datasets. 


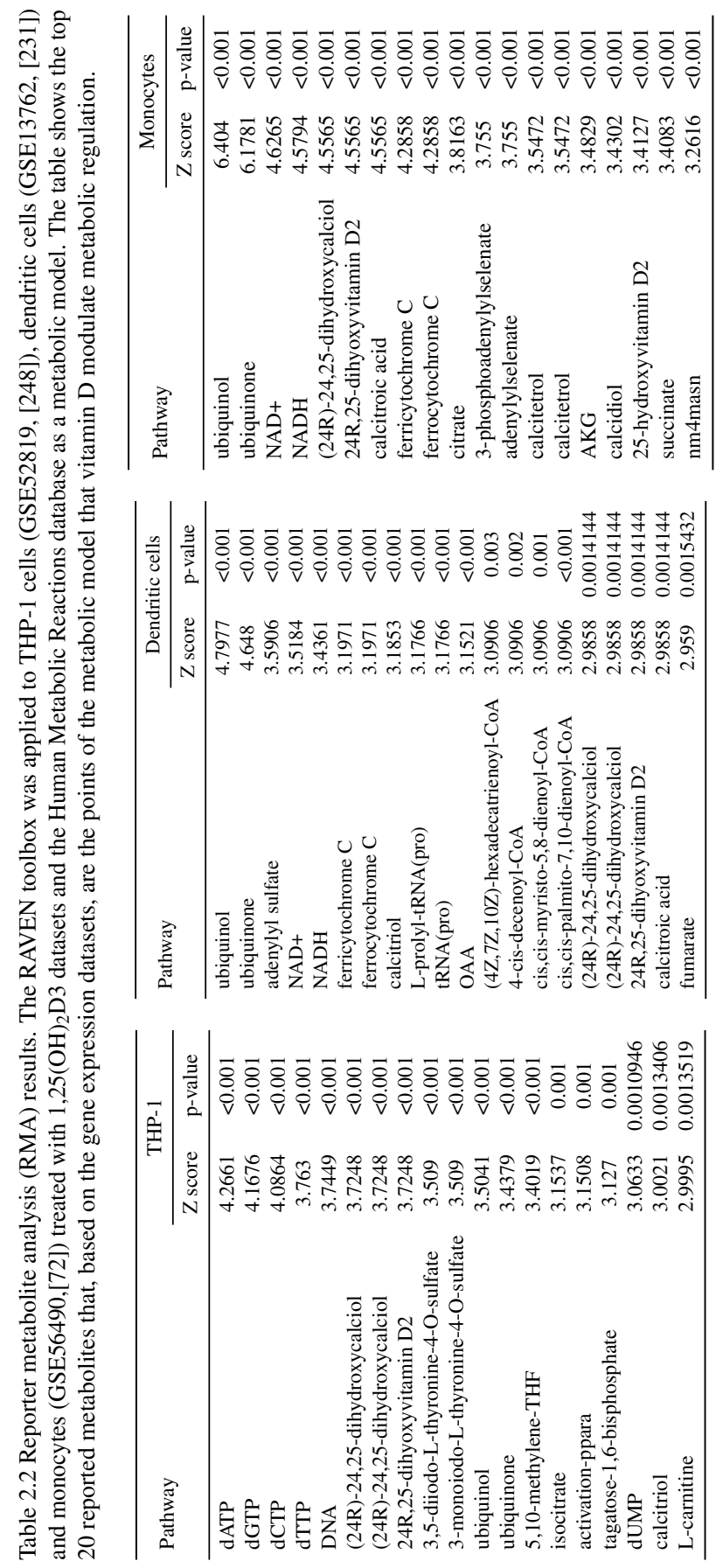




\subsubsection{Extension of the PI3K/Akt/mTOR-vitamin D3 signaling pathway with approved drugs, validated microRNAs and curated path- ways}

The WikiPathways PI3K/Akt/mTOR-vitamin D3 signaling pathway (https://www.wikipathways. org/index.php/Pathway:WP4141), created based on Mathieu's model proposed for the action mechanism of $1,25(\mathrm{OH})_{2} \mathrm{D} 3$ in human monocyte-derived dendritic cells [72], was extended with existing biological knowledge on gene-pathway, drug-gene and mature microRNA-gene interactions. The interactions between genes in the pathway with both approved drugs and experimentally validated microRNAs are shown in (figure 2.3). Moreover, the human curated pathways containing one or both nuclear receptors VDR and RXRA are overlaid on the network. The complete extended network is available online at the NDEx-The Networks Data Exchange [192]. The combination of various interaction data sources enables the integrated analysis of which drug targets are present, whether they are affected by microRNAs and in which processes they occur. The VDR which is activated upon binding of $1,25(\mathrm{OH})_{2} \mathrm{D} 3$ is central in the effect of vitamin D3. The networks show several compounds that have VDR as a target. One of these is ergocalciferol which belongs to the category of bone density conservation agents. Interestingly, VDR is also a target for three validated mature microRNAs, hsa-miR-27b-3p, hsa-miR-124-3p and hsa-miR-125b-5p. Finally, VDR is present in seven pathways of which four contain RXRA as well, like the vitamin D metabolism and nuclear receptor pathways. 


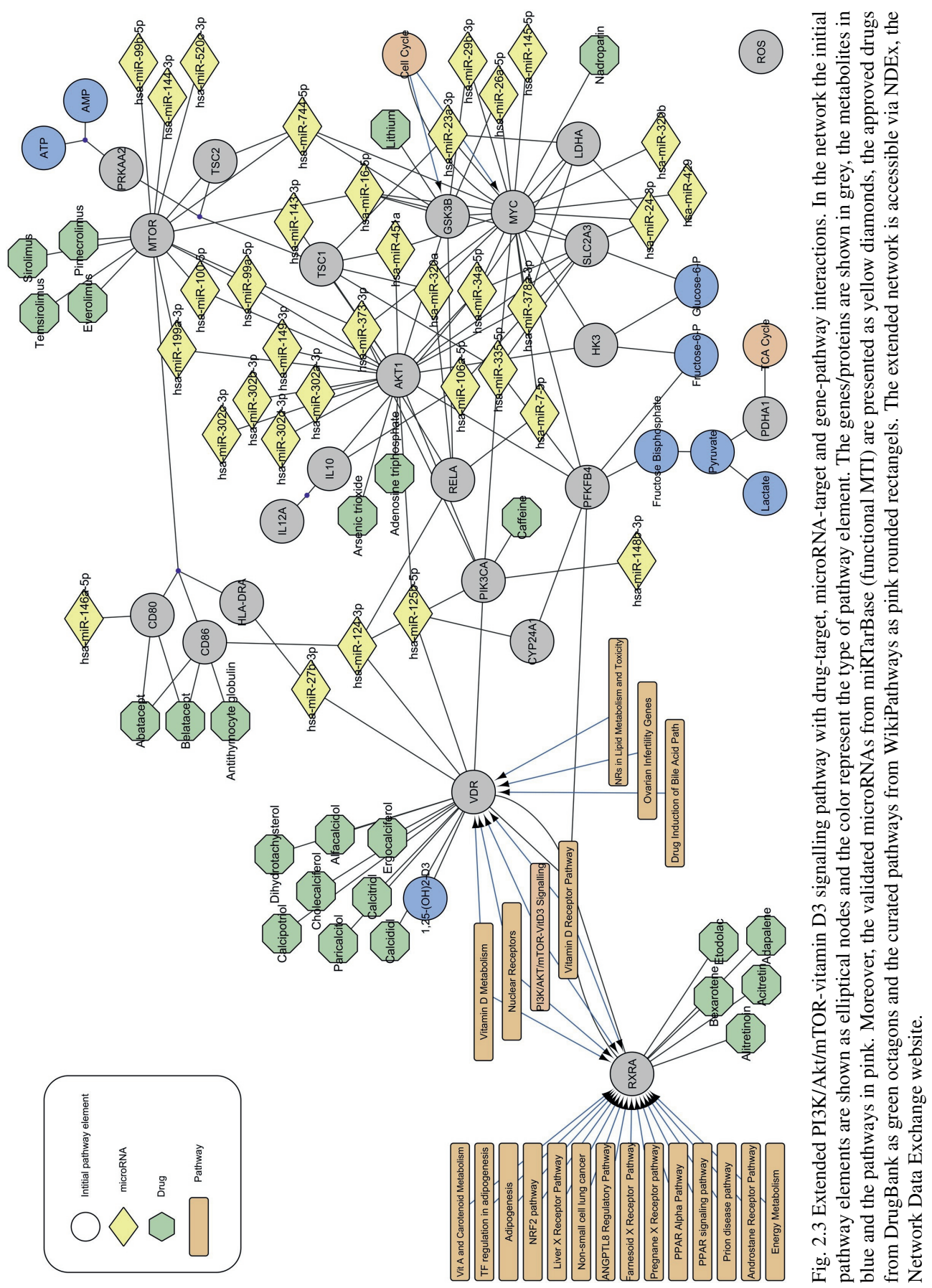




\subsection{Conclusion}

Open bioinformatics tools can be brought together in one pipeline that allows the user to analyse transcriptomic data in a friendly and accessible workflow. This review explains various ways of functional interpretation that can be extracted from gene expression datasets applying this bioinformatic workflow. Integration of these pathways at many different levels highlights altered pathways, identifies critical regulatory points in metabolism and links these bio-molecules to the genes overrepresented in "omic" datasets. Finally, the results are presented in explanatory diagrams and networks that interconnect biological components that contextualizes statistical data that shows modulation of gene expression. 


\section{Chapter 3}

\section{Transcriptomic analysis of vitamin D responses in uterine and peripheral NK cells}

Published in Reproduction in 2019, DOI: 10.1530/REP-18-0509

Tamblyn JA ${ }^{1,2,3}$, Jeffery LE ${ }^{1}$, Susarla ${ }^{1}$, Lissauer DM ${ }^{1,2}$, Coort SL ${ }^{4}$, Garcia AM ${ }^{1,4}$, Knoblich $\mathrm{K}^{5}$, Fletcher $\mathrm{AL}^{5}$, Bulmer $\mathrm{JN}^{6}$, Kilby $\mathrm{MD}^{1,2,3,7}$, Hewison $\mathrm{M}^{1,3}$

${ }^{1}$ Institute of Metabolism and Systems Research, The University of Birmingham, Birmingham, B15 2TT, United Kingdom

${ }^{2}$ Centre for Women's \& Newborn Health, Birmingham Health Partners, Birmingham Women's \& Children's Foundation Hospital, Edgbaston, Birmingham, UK

${ }^{3}$ Centre for Endocrinology, Diabetes and Metabolism, Birmingham Health Partners, Birmingham, $U K$

${ }^{4}$ Department of Bioinformatics - BiGCaT, NUTRIM School of Nutrition and Translational Research in Metabolism, P.O. Box 616, Maastricht University, Maastricht, the Netherlands ${ }^{5}$ Biomedicine Discovery Institute, Monash University, Clayton, Victoria, Australia,

${ }^{6}$ Reproductive and Vascular Biology Group, Institute of Cellular Medicine, Newcastle University, Newcastle upon Tyne, UK and

${ }^{7}$ Fetal Medicine Centre, Birmingham Women's \& Children's Foundation Trust, Edgbaston, Birmingham, UK 


\subsection{Abstract}

Vitamin D deficiency is prevalent in pregnant women and is associated with adverse pregnancy outcomes, in particular disorders of malplacentation. The active form of vitamin $\mathrm{D}, 1,25$-dihydroxyvitamin $\mathrm{D} 3\left(1,25(\mathrm{OH})_{2} \mathrm{D} 3\right)$, is a potent regulator of innate and adaptive immunity, but its immune effects during pregnancy remain poorly understood. During early gestation, the predominant immune cells in maternal decidua are uterine natural killer cells (uNK), but the responsivity of these cells to $1,25(\mathrm{OH})_{2} \mathrm{D} 3$ is unknown despite high levels of $1,25(\mathrm{OH})_{2} \mathrm{D} 3$ in decidua. Transcriptomic responses to $1,25(\mathrm{OH})_{2} \mathrm{D} 3$ were characterised in paired donor $\mathrm{uNK}$ and peripheral natural killer cells $(\mathrm{pNK})$ following cytokine (CK) stimulation. RNA-seq analyses indicated 911 genes were differentially expressed in CK-stimulated uNK versus CK-stimulated pNK in the absence of $1,25(\mathrm{OH})_{2} \mathrm{D} 3$, with predominant differentially expressed pathways being associated with glycolysis and transforming growth factor $\beta$ (TGF $\beta$ ). RNA-seq also showed that the vitamin D receptor (VDR) and its heterodimer partner retinoid $\mathrm{X}$ receptor were differentially expressed in CK-stimulated UNK vs CK-stimulated pNK. Further analyses confirmed increased expression of VDR mRNA and protein, as well as VDR-RXR target in CK-stimulated UNK. RNA-seq analysis showed that in CK-stimulated pNK, 1,25(OH) $)_{2} \mathrm{D} 3$ induced 38 and suppressed 33 transcripts, whilst in CK-stimulated uNK $1,25(\mathrm{OH})_{2} \mathrm{D} 3$ induced 46 and suppressed 19 genes. However, multiple comparison analysis of transcriptomic data indicated that $1,25(\mathrm{OH})_{2} \mathrm{D} 3$ had no significant overall effect on gene expression in either CK-stimulated $\mathrm{pNK}$ or $\mathrm{uNK}$. These data indicate that CK-stimulated uNK are transcriptionally distinct from pNK and, despite expressing abundant VDR, neither pNK nor uNK are sensitive targets for vitamin D.

\subsection{Introduction}

Human implantation and placental development present a unique challenge for the maternal immune system [232]. From embryonic implantation, semi-allogeneic fetal extravillous trophoblast cells invade the maternal decidua and inner myometrium (including remodelling the spiral arteries) facilitating normal haemochorial placentation. Throughout these actions, the materno-fetal immune function remains active, with diverse mechanisms mounted to support fetal development and placentation [2][66]. From early pregnancy, a high proportion of resident leukocytes, displaying unique phenotypes and functions reside within the uteroplacental interface [31]. CD56bright uterine natural killer cells (uNK) (60-70\%) are predominant, appearing phenotypically distinct from CD56dim NK cells in peripheral blood (pNK) $(<10 \%$ total circulating immune cell population). In addition to the classical pro- 
cytotoxic, anti-tumorigenic and anti-viral effects associated with pNK [123], uNK have also been shown to play a pivotal role in embryonic implantation and haemochorial placental development [139].

Regulation of uNK function involves a complex network of factors [230], and we have postulated that vitamin D plays a role in this process. Early in pregnancy, maternal concentrations of the active form of vitamin $\mathrm{D}, 1,25(\mathrm{OH})_{2} \mathrm{D} 3$, increase dramatically [235]. Moreover, the enzyme that catalyses synthesis of $1,25(\mathrm{OH})_{2} \mathrm{D} 3$ from precursor 25-hydroxyvitamin D3 (25(OH)D3), CYP27B1 [88][257][265] as well as the VDR for 1,25(OH) $)_{2}$ D3 [265], are abundantly expressed by both maternal decidua (stromal and immune cells) and fetal trophoblast [67]. The precise targets for decidual $1,25(\mathrm{OH})_{2} \mathrm{D} 3$ are yet to be defined, but may include the effects upon VDR-expressing decidual immune cells [265]. The aim of the current study was to characterise ex vivo the effects of $1,25(\mathrm{OH})_{2} \mathrm{D} 3$ on gene expression in activated uNK from first-trimester decidual tissue and to contrast these changes with effects of $1,25(\mathrm{OH})_{2} \mathrm{D} 3$ on paired $\mathrm{pNK}$.

\subsection{Methods}

\subsubsection{Ethical approval}

Paired decidual and peripheral blood samples were obtained from healthy pregnant women undergoing first trimester elective surgical termination of pregnancy (TOP) $(<12$ weeks (w) gestational age (GA); range 7-11+2 w) following written informed consent (2014-2017; NHS REC approval 06/ Q2707/12 and 13/WM/0178). GA was determined by ultrasound measurement of crown rump length and relevant anonymised patient demographics obtained as summarised (Supplementary Table 1, see section on supplementary data given at the end of the article).

\subsubsection{Isolation of $\mathrm{NK}$ cells from peripheral blood and decidua}

CD56+ cell-enriched isolates were prepared by modification of a previously published method [140]. Briefly, whole decidual tissue was minced and DNase/collagenase treated. Lymphocyte purification was then carried out by density gradient centrifugation (Ficoll Plus, GE Healthcare Life Sciences). For initial cell-surface marker analysis, anti-CD56-reactive magnetic bead separation (MidiMACS, Miltenyi Biotec) was performed to obtain CD56+ cells. 


\subsubsection{Cell culture}

Paired uNK and pNK were cultured in complete cell culture medium (Penstrep $100 \mu \mathrm{g} / \mathrm{ml}$, L-glutamine $2 \mathrm{mM}$, RPMI 1640, fetal calf serum (10\%) (Sigma-Aldrich) at $37^{\circ} \mathrm{C}$ and $5 \%$ $\mathrm{CO}_{2}$ for $24 \mathrm{~h}$, as either unstimulated controls or with cytokine (CK) stimulation using recognised NK cell co-activators: IL-2 (50 IU/mL), IL-12 (10 ng/mL), and IL-15 (10 $\mathrm{ng} / \mathrm{mL})$. Unstimulated and $\mathrm{CK} \mathrm{uNK}$ and $\mathrm{pNK}$ were cultured in the presence and absence of $1,25(\mathrm{OH})_{2} \mathrm{D} 3(10 \mathrm{nM})$ (Enzo Lifesciences, Exeter, UK) co-treatment.

\subsubsection{Flow cytometric analysis of CD69 and VDR}

Expression of VDR (D6 anti-VDR; Santa Cruz Biotechnology) and CD69 (anti-CD69; BD Biosciences), a recognised surface marker of NK cell activation [49] was assessed in paired pNK and uNK following $24 \mathrm{~h}$ culture in the presence and absence of CK stimulation with or without $1,25(\mathrm{OH})_{2} \mathrm{D} 3$. Briefly, cultured cells were harvested, washed in PBS and incubated with live/dead fixable discrimination dye (Thermo Fisher) on ice for $30 \mathrm{~min}$. Cells were washed with PBS, incubated for 30 min with surface primary mAb at $4{ }^{\circ} \mathrm{C}$ and then re-washed. For intracellular VDR analysis, a protocol optimised from Bendix et al. was used [17]. Briefly, following surface staining, cells were fixed for $20 \mathrm{~min}$ in $100 \mu \mathrm{L} 4 \%$ PFA, washed twice in $2 \mathrm{~mL} 2 \%$ FCS-PBS and incubated for $30 \mathrm{~min}$ on ice in $75 \mu \mathrm{L} 5 \%$ FCS $0.5 \%$ Triton X solution (Sigma-Aldrich). Cells were then incubated with anti-VDR $\mathrm{mAb}$ for $30 \mathrm{~min}$, washed with $2 \%$ FCS-PBS and re-incubated with anti-mouse IgG2a APC secondary $\mathrm{mAb}$ under the same conditions. Cells were then re-washed twice in $2 \%$ FCS-PBS followed by PBS and stored at $4{ }^{\circ} \mathrm{C}$ prior to analysis. Flow cytometry data were collected using a multi-channel Dako Cyan flow cytometer (Beckman Coulter). Data were analysed using Flow Jo V10 (Tree star, Inc., Ashland, USA), gating live CD45+ CD56+ NKp46+ cells (Supplementary Fig. 2). The specificity of the D6 anti-VDR mAb in VDR positive control cells (activated T cells) was demonstrated using flow cytometry, immunoprecipitation and chromatin immunoprecipitation (Supplementary Fig. 3).

\subsubsection{Confocal imaging of VDR in CD56+ pNKs}

$\mathrm{CD} 56+\mathrm{pNKs}$ were isolated and stimulated overnight with $\mathrm{CK}$ in the presence of $1,25(\mathrm{OH})_{2} \mathrm{D} 3$ as described above. Cells were surface-labelled for CD56 using FITC-anti-CD56 (clone REA196, Miltenyi Biotec), then fixed and permeabilised for VDR labelling with mouse anti-human VDR (D6 clone, Santa Cruz Biotechnology) or mouse IgG2a isotype control according to the protocol described above for flow cytometry but with goat anti-mouse 
IgG2a-594 and goat-anti-FITC-488 (molecular probes, Fisher Scientific) secondary labelling for $30 \mathrm{~min}$ in the presence of Hoechst $33342(10 \mu \mathrm{g} / \mathrm{mL}$; Fisher Scientific). After a final wash, cells were re-suspended in ProlongR Gold antifade reagent (Molecular Probes, Cell Signalling Technologies) and placed under coverslip on charged microscope slides. Edges were sealed with nail varnish and 22 -slice $\mathrm{Z}$ stack images of cells at $63 \times$ magnification, resolution $1024 \times 1024$ pixels per image were captured using the Zeiss LSM 880, Axio Observer confocal microscope and studied with Zen(2012) software (Supplementary Fig. 4).

\subsubsection{Quantitative real-time PCR (qRT-PCR) analysis}

Total RNA was extracted as described previously [113]. cDNA was prepared by reverse transcription TaqMan Reverse Transcription Reagents Kit (Applied Biosystems, Roche) as per manufacturer's instructions. qRT-PCR was performed on an ABI 7500 qPCR machine using TaqMan assays from Applied Biosystems for VDR (Hs00172113_m1) and multiplexed with VIC labelled 18S rRNA (4319413E) (Applied Biosystems, Roche). Paired uNK and pNK gene expression following $24 \mathrm{~h}$ culture in the presence and absence of CK stimulation with and without $1,25(\mathrm{OH})_{2} \mathrm{D} 3$ co-treatment was measured. Relative expression compared to paired unstimulated pNK was assessed using fold-change calculated by the $2-\Delta \Delta \mathrm{Ct}$ method. All statistical analyses were carried out using Sigmaplot 9.0 software (Systat Inc., San Jose, CA, USA). Experimental means were compared statistically using one-way ANOVA, with the Holm-Sidak method used as a post hoc multiple comparison procedure. Statistical analyses for RT-qPCR analyses were carried out using raw $\Delta \mathrm{Ct}$ values.

\subsubsection{RNA sequence analysis (RNA-seq)}

Total RNA was extracted using the RNeasy Micro Kit and RNeasy MinElute spin columns (Qiagen), as per manufacturer's instructions, eluted in $15 \mu \mathrm{L}$ RNAse-free water, and immediately stored at $-80^{\circ} \mathrm{C}$ prior to library preparation and sequencing (Source Bioscience Facilities, Nottingham, UK). Following initial QC check using the Agilent BioAnalyzer 2100 (Agilent) cDNA library preparation was performed using Switching Mechanism at the 5' end of RNA Template (SMART)er Stranded Total RNA-seq Kit - Pico (Clontech, Mountain View) $($ RNA range $=p g$ ). Random primers and locked nucleic acid (LNA) technology were utilised to optimise total coverage. Illumina Adapters and Indexes (barcode markers) (Illumina, Inc.) were added at PCR1 with rRNA cleaved by ZapR in the presence of R-Probes (mammalian specific). The non-cleaved fragments underwent further enrichment in PCR2 cycle. All libraries were QC validated using the Agilent BioAnalyzer 2100 (Santa Clara) to confirm index samples amplified concentration and size distribution. The libraries were 
pooled and re-validated to assess molarity and size distribution prior to loading (1.8 $\mathrm{pM}$ concentration) onto a High Output NextSeq 500 Flow Cell pv2. (Illumina) across four lanes for RNA-seq using 75-base-pair (bp) paired-end reading, generating 800 million total reads with an average of 25 million per sample. Raw data were returned in the FastQ Phred+33 (Illumina 1.9) format.

\subsubsection{Bioinformatics and pathway analysis}

Bioinformatics analysis of RNA-seq analyses was performed at the University of Birmingham, UK using Partek Flow Software (Partek, St Louis, Missouri, USA). Data were trimmed for adapter sequences and pre-alignment QA/QC was performed before being mapped (aligned) using STAR-2.4.1d [59]. Post-alignment QA/QC was performed with a coverage report generated. Reads were quantified to transcriptome hg38_RefSeq (Genome Reference Consortium GRCh38) and normalised using quantile normalisation. Median total aligned reads were 71567649 (IQR; 64250828-74820738), representing 85.7\% (84.5-87.5\%) coverage. Differential gene expression was defined by a cut-off fold-change of $<-1.5$ and $>$ $1.5, \mathrm{P}$ value $\leq 0.05$ and false discovery rate (FDR)-step up $\leq 0.05$. P values were calculated using F-statistics, which calculates variance within samples. Three analyses were assessed: (i) $\mathrm{CK}$ uNK versus (vs) $\mathrm{CK} \mathrm{pNK}$; (ii) $\mathrm{CK}$ uNK vs $\mathrm{CK} \mathrm{uNK}+1,25(\mathrm{OH})_{2} \mathrm{D} 3$; (iii) $\mathrm{CK} \mathrm{pNK}$ vs $\mathrm{CK}$ pNK $+1,25(\mathrm{OH})_{2} \mathrm{D} 3$.

Characterisation of common biological processes in the CK-activated uNK and pNK groups was carried out using PathVisio (Version 3.2.4), an open-source pathway analysis and visualisation software tool for biological data analysis, using previously described protocols [81]. Briefly, the analysis was performed using the 11164 raw gene counts obtained, with corresponding NCBI ID and symbol from the HUGO Gene Nomenclature Committee (HGNC) identifiers. A $\mathrm{P}$ value $<0.05$ was applied to determine differentially expressed genes. The $\mathrm{Z}$ score, which signifies whether the total number of genes in the pathway are over-represented ( $Z$ score $>1.96$ ) compared to the complete data set, was then calculated. The curated human pathway collection from the online-pathway repository WikiPathways [135] including the Reactome [25] collection (downloaded May 2017) was used. 


\subsection{Results}

\subsubsection{Comparison of gene expression in cytokine (CK)-stimulated pNK and $\mathrm{uNK}$ cells}

Principal component analysis (PCA) of unbiased genome-wide RNA-seq analyses revealed wide variance in the transcriptional profiles of CK-stimulated uNK and pNK (Figure 3.1 A). In both cell types, co-treatment with $1,25(\mathrm{OH})_{2} \mathrm{D} 3$ did not result in PCA separation of transcriptional profiles from their respective CK controls. Volcano plots of differentially expressed genes in CK-stimulated $\mathrm{uNK}$ relative to CK-stimulated $\mathrm{pNK}$ using a cut-off of $\mathrm{p} \leq$ 0.05 and fold-change $\leq-1.5$ or $\geq 1.5$ showed 1098 upregulated genes in CK-stimulated uNKs (red) and 1188 downregulated genes (green), with those not significantly different in grey (n = 11163) (Figure 3.1 B). Adjustment for FDR identified 911 transcripts to be differentially expressed between CK-stimulated uNK and pNK, with 422 down-, and 489 upregulated in CK-stimulated uNK relative to $\mathrm{CK}$-stimulated $\mathrm{pNK}$ ( $\mathrm{P}$ value $\leq 0.05$; $\log 2$ fold-change $\leq-1.5$ or $\geq 1.5$, FDR step-up $\leq 0.05$ ) (Figure 3.1 C). Significantly regulated genes in CK-stimulated uNK relative to CK-stimulated pNK included upregulation of NCAM1 (CD56) (fold-change 5.29) and downregulation of FCG3RA (CD16A) (fold-change -14.57), both of which are well-recognised differentially expressed markers for CD56bright NK versus CD56dim pNK subsets [123] [196]. Interestingly, granzyme (GZMK) (fold-change (7.83)) and CD96 (2.53), which permits adhesive interactions and modulates responses between activated $\mathrm{T}$ and $\mathrm{NK}$ cells [85], were both upregulated in CK-stimulated uNK. CD276 (7.71), a regulator of T cell cytotoxicity [189], was also upregulated in CK uNK. 


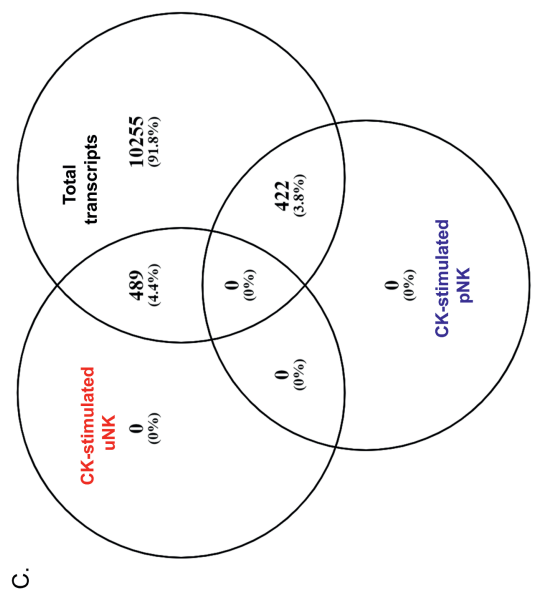

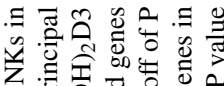

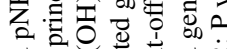

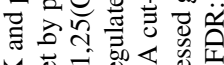

光芯范苛灾这

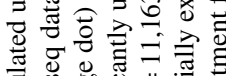

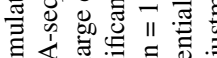

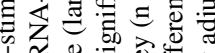

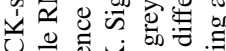

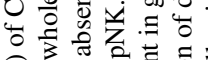

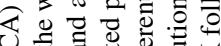

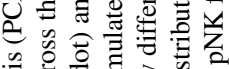

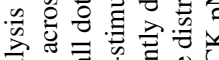

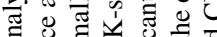

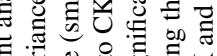

过 过

웅 记

है ठै匕

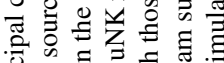

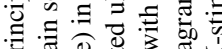

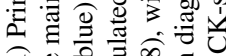

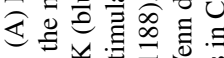

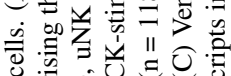

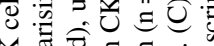

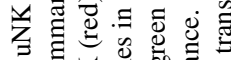

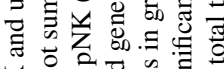

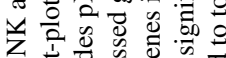

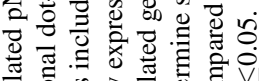

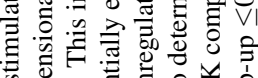

$\dot{\infty}$

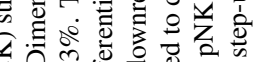

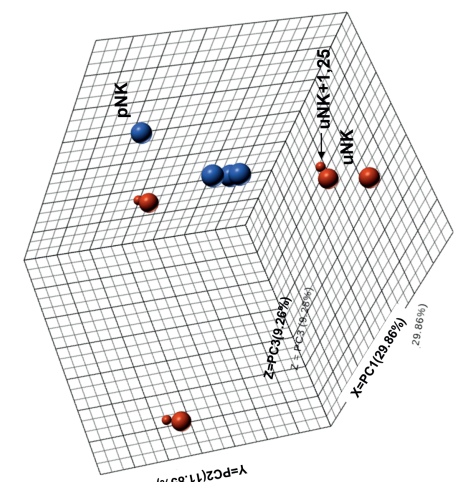

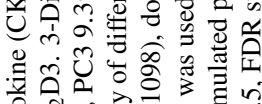

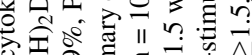

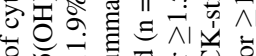

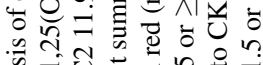

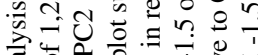

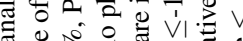

๘ั

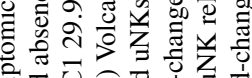

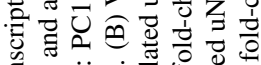

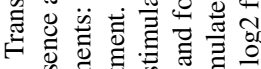

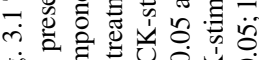

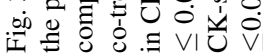


To better delineate the differences in transcript expression between CK-stimulated uNK and pNK, complementary pathway analysis was performed on FDR-adjusted differentially expressed data using WikiPathways (WP) workflows. A broad spectrum of enriched canonical pathways was identified, with significantly enriched pathways ranked from high to low $\mathrm{Z}$ score. Pathways significantly enriched based on a $\mathrm{Z}$ score $\geq 1.96$ are shown in table 3.1. Overall, 29 WP pathways were significantly enriched in CK-stimulated uNK relative to CK-stimulated pNK, including pathways related to hypoxia-inducible factor (HIF) $1 \alpha$ and peroxisomal proliferator-activated receptor $(\mathrm{PPAR}) \gamma$ regulation of glycolysis, and transforming growth factor (TGF) $\alpha$ signalling (Supplementary Figures. 5A-5S).

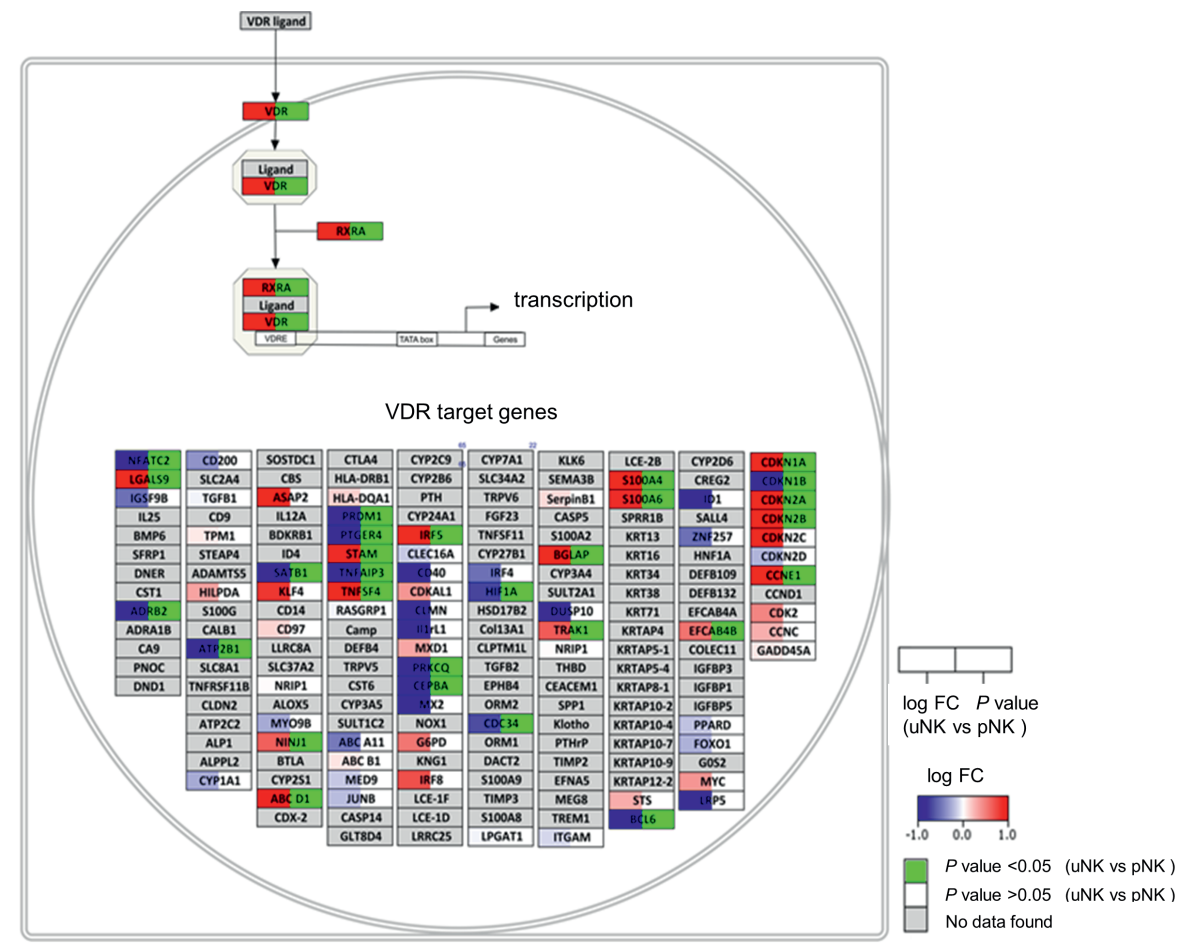

Fig. 3.2 Pathway analysis of vitamin D receptor (VDR) signallingrelated genes in CK-stimulated uNK versus CK-stimulated pNK. Visualisation of genes with enhanced (red) or suppressed (blue) expression in CKstimulated uNK versus CK-stimulated pNK. Individual genes are shown in boxes and box colour split into two parts, (1) the $\log 2$ fold-change in the left part of the box (blue downregulated, white not changed, red upregulated) and (2) $\mathrm{P}$ value for statistical significance is shown in the right part of the box (green when significant). Pathway elements not assessed in the dataset are shown in grey. 


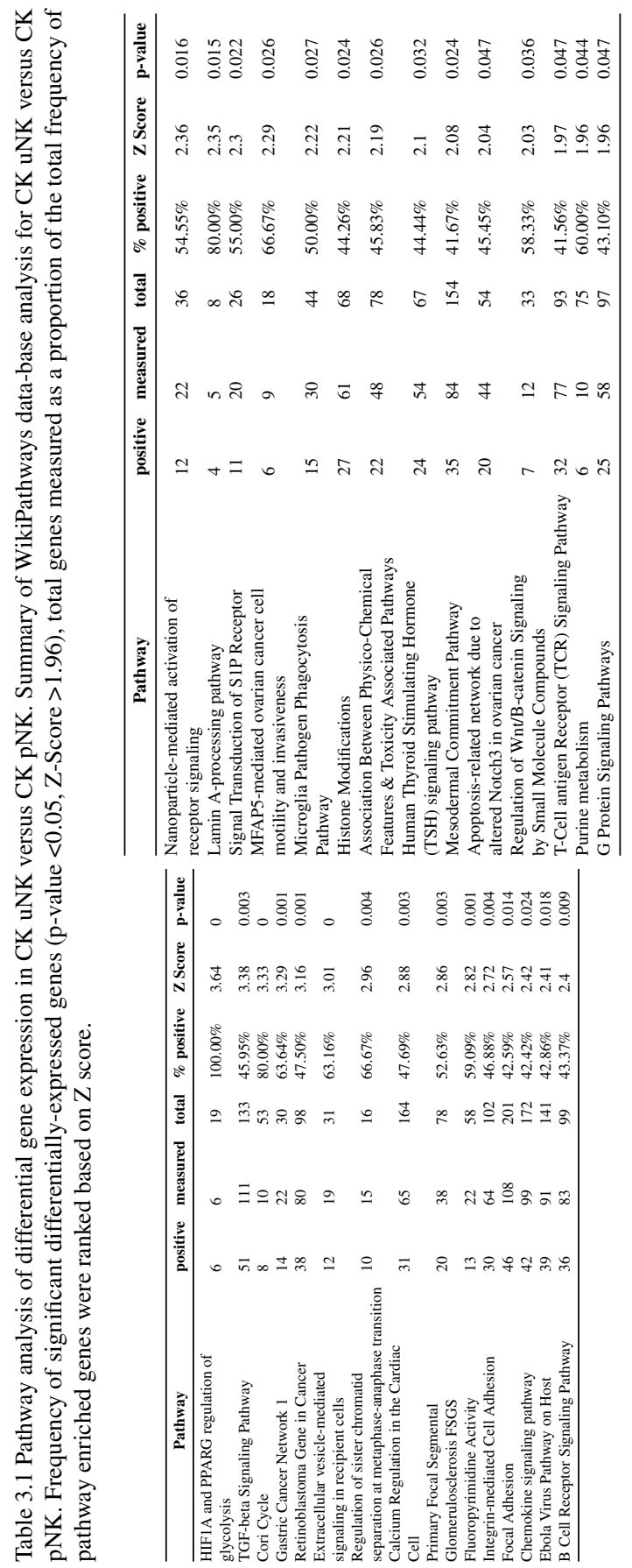




\subsubsection{VDR expression in uNK is upregulated following cytokine stimu- lation}

RNA-seq analysis revealed that VDR mRNA expression is significantly upregulated in CKstimulated uNK (fold-change 8.56) relative to CK-stimulated pNK. Analysis of differentially expressed pathways in CK-stimulated uNK versus CK-stimulated pNK in Supplementary dataset 1 also showed enhanced expression of pathways associated with VDR and its heterodimer partner retinoid $X$ receptor $(R X R)(Z$ score $1.83, p=0.07)$ (Figure 3.2), as well as non-genomic actions of $1,25(\mathrm{OH})_{2} \mathrm{D} 3(\mathrm{Z}$ score 1.88, $\mathrm{P}=0.062)$ (Figure 3.5). This suggests that capacity for $1,25(\mathrm{OH})_{2} \mathrm{D} 3-\mathrm{VDR}$ responses is elevated in $\mathrm{CK}$-stimulated uNK relative to CK-stimulated $\mathrm{pNK}$.

Studies were therefore carried out to further characterise VDR expression in uNK and pNK. Flow cytometry showed that pNK and uNK expressed similar levels of the NK marker NKp46. Analysis of CD56 expression showed that uNK cells were characterised by more distinct populations of CD56bright and CD56dim cells than pNK (Figure 3.3 A). Both CD56 and NKp46 showed no change in response to either $\mathrm{CK}$ stimulation or $1,25(\mathrm{OH})_{2} \mathrm{D} 3$ treatment in either uNK or pNK cells for median fluorescence intensity (MFI) (Figure 3.3 A) or frequency of expression (data not shown). As shown in (Figure 3.3 B and C), CK stimulation significantly upregulated expression of the activation marker CD69 in both uNK (median 47.9\% IQR 31.5-56.6 to 68.1\%; 48.9-76.4) and pNK (17.7\%; 11.5-20.3 to 70.5\%; 57.4-72.8), but treatment with $1,25(\mathrm{OH})_{2} \mathrm{D} 3$ had no significant effect on CD69 expression in either the unstimulated or CK-stimulated uNK or pNK populations (Figure 3.3 C).

Without stimulation the frequency of NK cells that expressed VDR was low for both uNK (36.2\%; 33.1-36.5) and pNK (15.8\%; 5.81-61.6). CK stimulation significantly increased VDR frequency in uNK $(74.2 \% ; 65.6-81.3)$, although the absolute level of VDR expression by each expressing cell (MFI) was not significantly increased (Figure 3.3 D). 1,25(OH) $)_{2} \mathrm{D} 3$ had no significant effect on VDR protein expression in either UNK or pNK in the presence or absence of CK stimulation (Figure 3.3 C). Expression of VDR mRNA was also significantly induced by $\mathrm{CK}$ stimulation in $\mathrm{uNK}(\mathrm{P}=0.0001)$ and $\mathrm{pNK}(\mathrm{P}=0.017)$. Interestingly, this effect was suppressed in both cell types by co-treatment with $1,25(\mathrm{OH})_{2} \mathrm{D} 3$ (Figure 3.3 E). Confocal imaging of CK-stimulated pNK cells (Supplementary Fig. 4) showed colocalisation of VDR protein with both DNA (Hoescht staining) and the plasma membrane (CD56), consistent with both membrane and nuclear localisation of VDR. 
A. NKp46 vs CD56
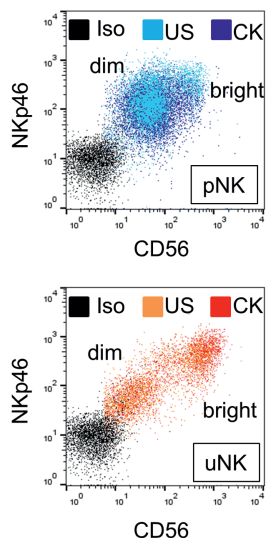

D.

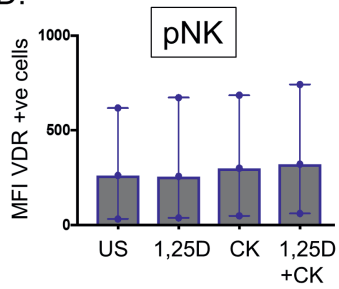

B. CD69 vs VDR
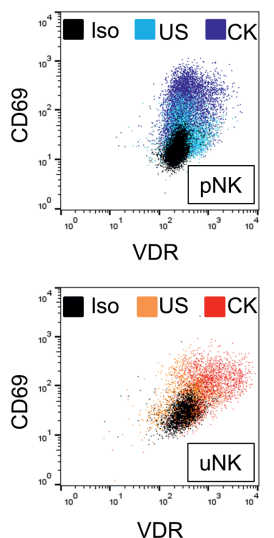

C.

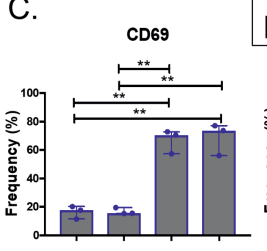

\section{pNK}
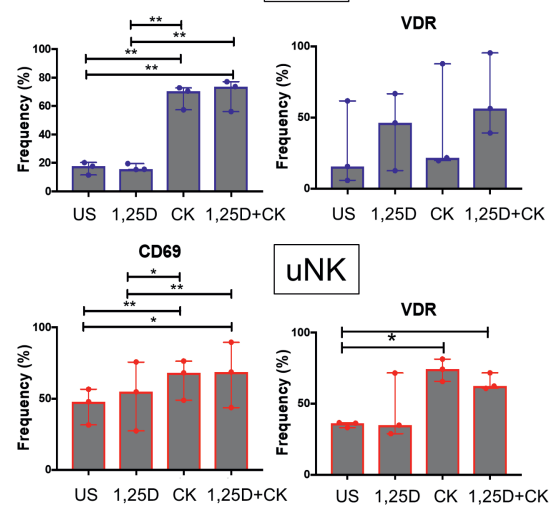

uNK

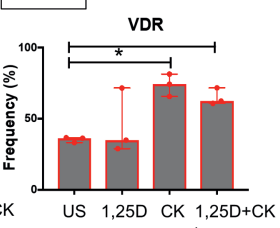

Fig. 3.3 Expression of VDR in paired peripheral blood and uterine natural killer cells. (A) Representative flow cytometry plots for expression of NKp46 and CD56 in isolated unstimulated (US) and cytokine (CK)-stimulated peripheral blood natural killer cells ( $\mathrm{pNK}$ ) and uterine natural killer cells ( $\mathrm{uNK}$ ) relative to isotype control (Iso). (B) Representative flow cytometry plots for expression of VDR and CD69 in US and CK-stimulated CD3-CD56+ uNK and pNK cells relative to Iso control. (C) Summary of VDR and CD69 surface protein expression in US- and CK uNK and -pNK cells in the presence or absence of $1,25(\mathrm{OH})_{2} \mathrm{D} 3\left(1,25(\mathrm{OH})_{2} \mathrm{D} 3\right)$. (D) Median fluorescence intensity (MFI) for VDR expression in pNK and uNK cells. (E) mRNA expression for VDR in isolated US and CK uNK and pNK cells in the presence and absence of $1,25(\mathrm{OH})_{2} \mathrm{D} 3$. Relative expression compared to US uNK cells; median value with bars denoting inter-quartile range. Effect of CK stimulation and $1,25(\mathrm{OH})_{2} \mathrm{D} 3$ was assessed by two-way ANOVA. Stars indicate level of significant difference between groups for which multiple comparisons analysis indicated significance $*<0.05, * *<0.01$. 


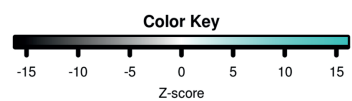

\section{Pathway Analysis}

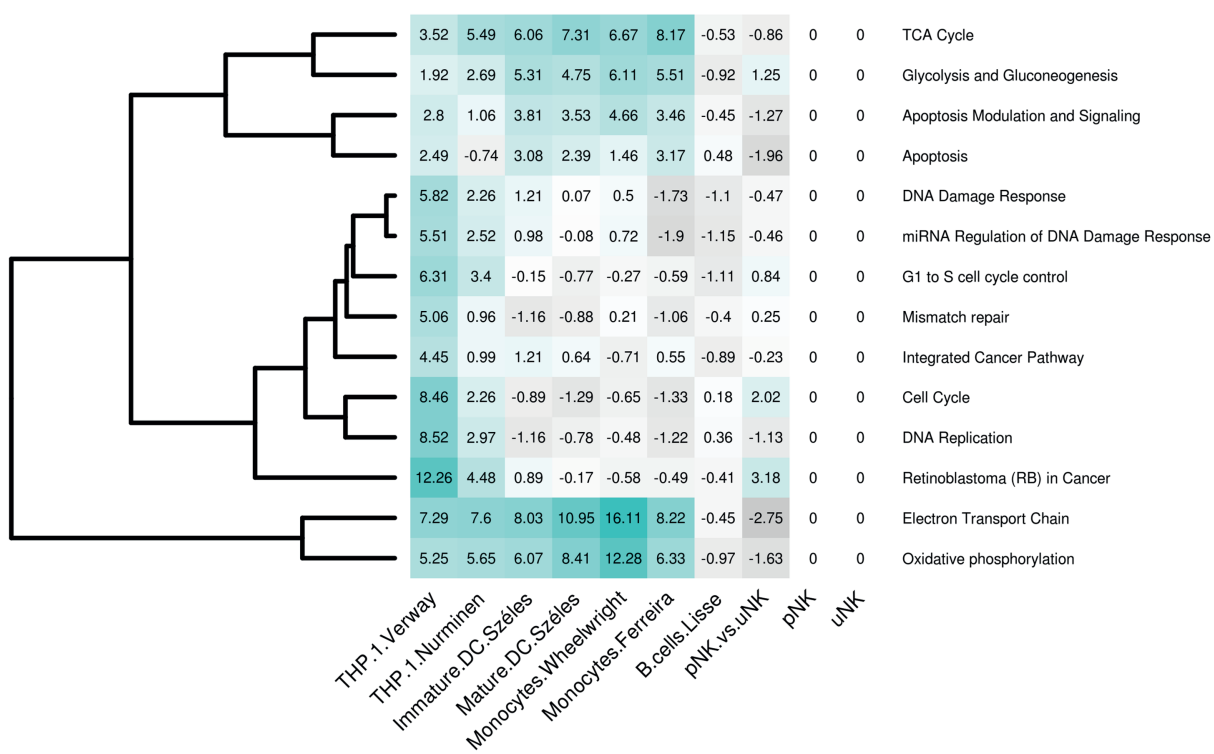

Fig. 3.4 Pathway analysis of transcriptomic responses to $1,25(\mathrm{OH})_{2} \mathrm{D} 3$-treated in THP-1 cells, dendritic cells, monocytes and $\mathrm{B}$ cells compared to effects in $\mathrm{pNK}$ and $\mathrm{uNK}$. Heat map representing Z-scores for pathways significantly altered by $1,25(\mathrm{OH})_{2} \mathrm{D} 3$ comparing pathway analysis on datasets from THP-1 cells, dendritic cells (DC), monocytes and B cells with effects of $1,25(\mathrm{OH})_{2} \mathrm{D} 3$ on CK-stimulated pNK and uNK. Z-scores $>1.96$ indicate that more genes are significantly altered in this pathway compared to the complete dataset. Genes differentially expressed between CK-stimulated uNK and pNK in the absence of $1,25(\mathrm{OH})_{2} \mathrm{D} 3(\mathrm{pNK}$ vs uNK) showed minimal overlap with the effect of $1,25(\mathrm{OH})_{2} \mathrm{D} 3$ on immune cells. The effects of $1,25(\mathrm{OH})_{2} \mathrm{D} 3 \mathrm{on}$ CK-stimulated pNK or uNK showed no commonality with the other immune cells. 


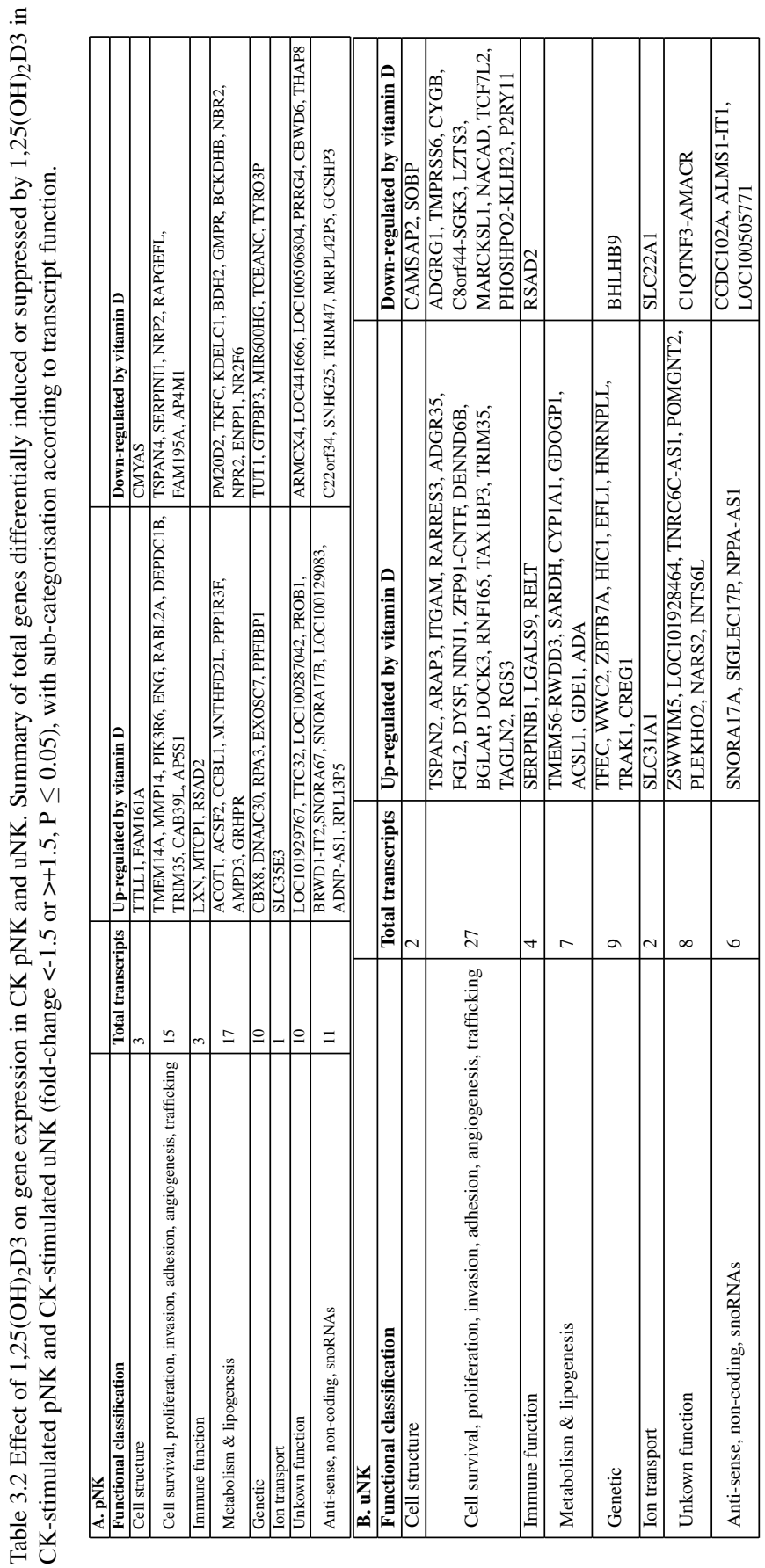




\subsubsection{Effects of $1,25(\mathrm{OH})_{2} \mathrm{D3}$ on gene expression in $\mathrm{CK}$-stimulated $\mathrm{pNK}$ and $\mathrm{uNK}$ cells}

When compared to CK-stimulated $\mathrm{pNK}$, CK-stimulated pNK treated with $1,25(\mathrm{OH})_{2} \mathrm{D} 3$, showed only 71 genes differentially expressed genes (fold-change $\pm 1.5 ; \mathrm{P} \leq 0.05$ ). Of these, $33(46.5 \%)$ were downregulated and $38(53.5 \%)$ upregulated by $1,25(\mathrm{OH})_{2} \mathrm{D} 3$. In CKstimulated uNK treated with $1,25(\mathrm{OH})_{2} \mathrm{D} 366$ genes were differentially expressed relative to CK stimulation of uNK alone. Of these, 46 (69.7\%) were down- and 19 (30.3\%) upregulated by $1,25(\mathrm{OH})_{2} \mathrm{D} 3$. In contrast to $\mathrm{pNK}, 1,25(\mathrm{OH})_{2} \mathrm{D} 3$ primarily targeted uNK genes implicated in cell processing, specifically cell adhesion, apoptosis, migration and angiogenesis ( $\mathrm{n}=27 ; 41.5 \%$ ) (table 3.2). Due to the relatively small number of genes differentially expressed in response to $1,25(\mathrm{OH})_{2} \mathrm{D} 3$ in either CK-stimulated $\mathrm{pNK}$ or $\mathrm{uNK}$, adjustment of data to incorporate fold-change \pm 1.5 , P value $\leq 0.05$, FDR step up $<0.05$ resulted in no significant effect of $1,25(\mathrm{OH})_{2} \mathrm{D} 3$ for either CK-stimulated uNK or CK-stimulated pNK. Furthermore, pathway analysis using non-adjusted differentially expressed gene data for either CK-stimulated uNK or CK-stimulated pNK cell treated with $1,25(\mathrm{OH})_{2} \mathrm{D} 3$ showed no significant enrichment of any specific pathways by $1,25(\mathrm{OH})_{2} \mathrm{D} 3$ (data not shown), and no commonality with any of the pathways that are known to be regulated by $1,25(\mathrm{OH})_{2} \mathrm{D} 3$ in other immune cells (Figure 3.4). 

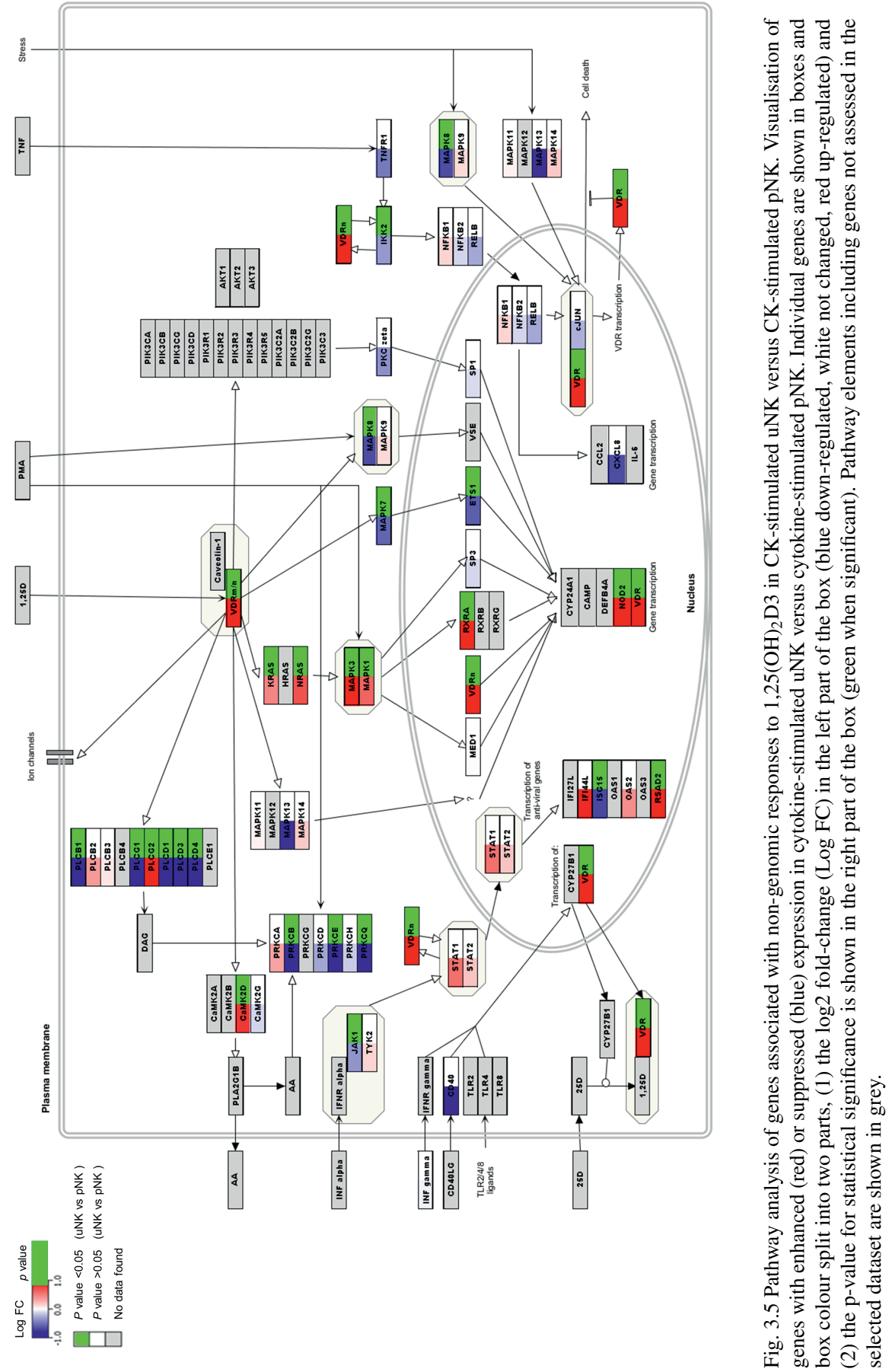
Title: HIF1A and PPARG regulation of

Last modified: $10 / 16 / 2013$

Organism: Homo sapiens
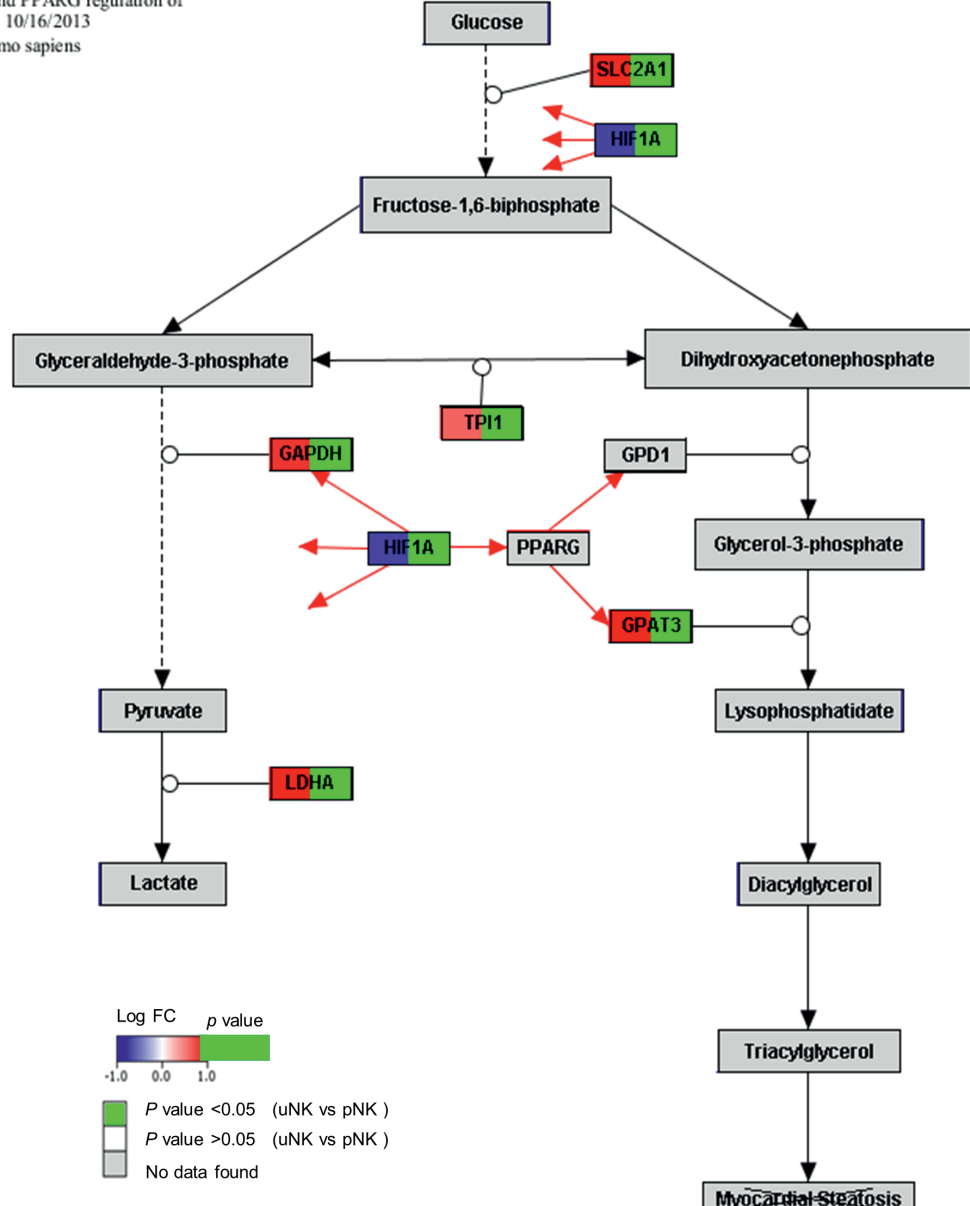

Fig. 3.6 Pathway analysis of HIF1A and PPARG regulation of glycolysis-related genes in CK-stimulated uNK versus CK-stimulated pNK. Pathway analysis of genes associated with non-genomic responses to $1,25(\mathrm{OH})_{2} \mathrm{D} 3$ in CK-stimulated uNK versus CK-stimulated pNK. Visualisation of genes with enhanced (red) or suppressed (blue) expression in cytokine-stimulated uNK versus cytokine-stimulated pNK. Individual genes are shown in boxes and box colour split into two parts, (1) the $\log 2$ fold-change (Log FC) in the left part of the box (blue down-regulated, white not changed, red up-regulated) and (2) the p-value for statistical significance is shown in the right part of the box (green when significant). Pathway elements including genes not assessed in the selected dataset are shown in grey. 


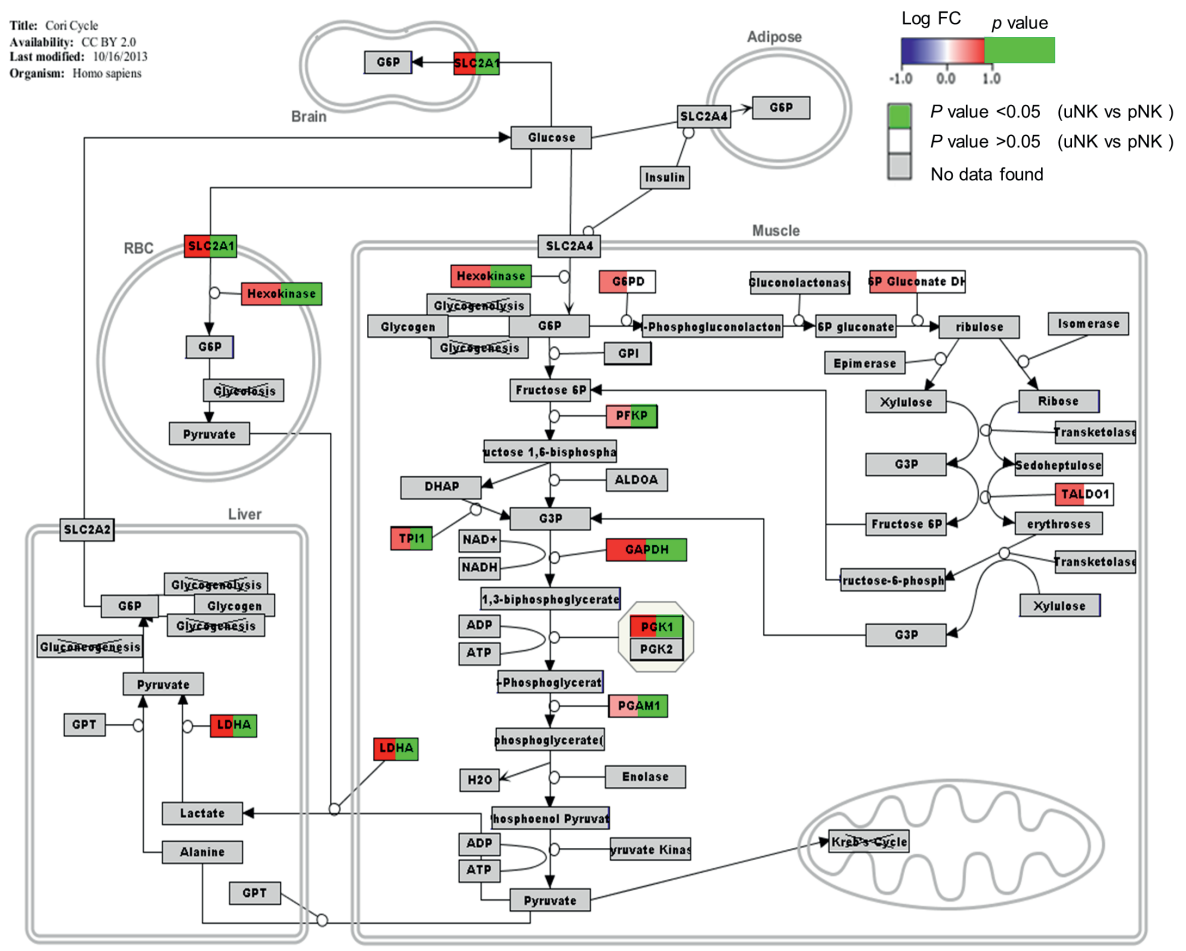

Fig. 3.7 Pathway analysis of the Cori cycle in CK-stimulated uNK versus CK-stimulated pNK. Pathway analysis of genes associated with non-genomic responses to $1,25(\mathrm{OH})_{2} \mathrm{D} 3$ in CK-stimulated uNK versus CK-stimulated pNK. Visualisation of genes with enhanced (red) or suppressed (blue) expression in cytokine-stimulated uNK versus cytokine-stimulated pNK. Individual genes are shown in boxes and box colour split into two parts, (1) the $\log 2$ fold-change (Log FC) in the left part of the box (blue down-regulated, white not changed, red up-regulated) and (2) the p-value for statistical significance is shown in the right part of the box (green when significant). Pathway elements including genes not assessed in the selected dataset are shown in grey. 


\subsection{Discussion}

Previous studies using DNA microarrays have described transcriptomic variations in CD56 bright pNK and CD56dim pNK cells, as well as CD56 bright uNK [123]. In the current study, pNK or uNK were not sub-categorised according to CD56 brightness, because CD56dim pNK predominate in peripheral blood (approximately 95\% of total pNK cell population), whilst CD56bright uNK are the prevalent NK type in decidua ( $>95 \%$ of total uNK population). These differences imply that uNKs are constitutively active. In agreement with this, we observed that uNK were larger with abundant perforin and granzyme. Importantly, no change in CD56 or NKp46 activation receptor expression was measured in $\mathrm{uNK}$ or pNK in response to $\mathrm{CK}$ stimulation or treatment with the active form of vitamin $\mathrm{D}, 1,25(\mathrm{OH})_{2} \mathrm{D} 3$. CD56bright NKs are considered efficient immunomodulatory cytokine producers, and only become cytotoxic following appropriate activation. However, in contrast to NK from most other sites, uNKs do not express the activating receptor CD16 which mediates antibody-dependent cellular cytotoxicity [196]. It appears that whilst uNKs maintain an intrinsic capacity to exert cytotoxic functions when specifically challenged, this function is regulated in normal early pregnancy [166]. Further undefined local mechanisms, such as cytokine or hormone secretion, or cross-talk with other immune cell types within the decidual microenvironment are also thought to suppress the potential lytic effects of uNKs [93].

In the current study, pro-inflammatory IL-2, IL-12 and IL-15 were utilised for CK challenge, as these are recognised NK cell activators, with both IL-12 and IL-15 constitutively expressed within the decidua from initial implantation [126] [268]. Although IL-2 concentrations appear low within normal decidua [115], significantly elevated IL-2 has been reported in the setting of malplacentation [84], where vitamin D deficiency is also more common [208]. The first aim of the current study was to compare the transcriptomic profiles of uNK versus pNK following immune activation by CK stimulation. The second objective of the study was to determine if CK-stimulated $\mathrm{uNK}$ and $\mathrm{pNK}$ were responsive to $1,25(\mathrm{OH})_{2} \mathrm{D} 3$ in a similar manner to that reported for other components of the immune system such as macrophages and T cells [3]. Since the effects of $1,25(\mathrm{OH})_{2} \mathrm{D} 3$ on NK cell function in decidua and maternal blood are still unclear, NK cell subsets were not further sub-classified.

In the current study, approximately 900 genes were differentially expressed in CK-stimulated uNK versus CK-stimulated $\mathrm{pNK}$, similar to previous DNA array analyses for unstimulated CD56dim pNK versus unstimulated CD56bright uNK [123]. In CK-stimulated uNK a comparable number of up-(429) and downregulated (422) genes were measured when adjusted for FDR. By contrast, in the absence of CK stimulation, it was reported that uNK almost 
exclusively exhibited enhanced transcription relative to pNK [123]. uNK responses to CK stimulation appear highly distinct from paired $\mathrm{pNK}$. In the circulation, $\mathrm{pNK}$ function as an early cytolytic response to tumours or viral infection [249] [43]). By contrast, consistent with previous reports, we observed that $\mathrm{uNK}$ are poorly cytolytic and express low levels of pNK cytokines such as interferon- $\gamma$ (INF- $\gamma$ ) and tumour necrosis factor- $\alpha$ (TNF- $\alpha$ ) [124][239] and appear less reactive with low CD107 (degranulation marker) expression relative to pNKs in response to $\mathrm{CK}$ challenge (data not published). The unique phenotype of uNK may reflect their adaptation within this unique maternal tissue microenvironment to aid decidualisation, embryonic implantation and subsequent fetal development [197].

Detailed pathway analysis verified that CK-stimulated uNK are phenotypically distinct from CK-stimulated pNK, with cell metabolism, signalling and processing, and cancer pathways consistently over-expressed in CK-stimulated uNK relative to CK-stimulated pNK. These observations are consistent with the known roles of tissue-resident uNK in placental development and fetal implantation. The significant inter-pathway transcript variance between CK-stimulated uNK and pNK underlines the complex diversity of uNK cell function. HIF1A and PPAR $\gamma$ regulation of glycolysis ( $Z$ score 3.64) was the most significantly differentially expressed pathway in CK-stimulated uNK (Figure 3.6). The Cori cycle, a lactate-glucose carbon recycling system between muscle and liver that decreases circulating lactate was also enriched in CK-stimulated uNK (Z score 3.33) (Figure 3.7). Genes associated with glycolysis and gluconeogenesis were also enhanced in CK-activated uNK ( $\mathrm{Z}$ score 1.83, $14 / 30$ genes, $P=0.067$ ), further underlining the active metabolic remodelling in uNK relative to pNK. During early pregnancy decidualisation is associated with enhanced glucose influx [77] [122]. Recent studies have shown that the decidua acts in a manner similar to that reported for tumours by generating energy via a high rate of glycolysis, even under aerobic conditions rather, than conventional oxidative phosphorylation [269]. This is referred to as a decidual Warburg-like glycolysis similar to that reported in tumours and was dependent on expression of HIF1 $\alpha$ and the lactate transporter protein (MCT4) [269]. Collectively, these data suggest that a key feature of activated uNK is to regulate glucose metabolism and lactate transport within the decidua. Dysregulation of glucose metabolism in the human uterus is associated with subfertility and aberrant implantation [122]. Glycolytic flux targets in uNK may therefore represent novel translational strategies to precisely time and/or therapeutically extend the window of receptivity in women at high risk for early pregnancy loss. Consistent with previous microarray analysis of first trimester uNK and adult non-paired pNK [252], the current study showed that CK-stimulated uNK are enriched for pathways associated with transcriptional regulation, which may serve to differentially regulate NK development and 
function in the decidual microenvironment. Whether $\mathrm{pNK}$ undergo significant transcriptional alterations following recruitment to the decidua or, alternatively, uNK represent an entirely distinct subset of NK cells remains unclear [94][238].

Of the total transcripts measured in CK-activated uNK and pNK, only $66(0.59 \%)$ and $71(0.64 \%)$ respectively were significantly regulated by $1,25(\mathrm{OH})_{2} \mathrm{D} 3(\mathrm{P} \leq 0.05$, fold-change \pm 1.5 ). Although transcriptomic analysis indicated that $\mathrm{CK}$ uNK and $\mathrm{CK}$ pNK respond differentially to $1,25(\mathrm{OH})_{2} \mathrm{D} 3$, this effect was statistically insignificant as the current analysis was not significantly powered to account for multiple comparisons (FDR step up <0.05). Sample size and participant heterogeneity are potential reasons for this. Intra-participant variability for $\mathrm{pNK}$ was high, whereas the uNK demonstrated comparatively low variability. The heterogeneity in pNK gene expression may be in part attributable to differences in gestational age, maternal age, ethnicity and smoking status (Supplementary Table 1), albeit $\mathrm{n}$ numbers are small. Cellular heterogeneity may also be a contributing factor to sample variability as recent single-cell RNA sequencing data indicates three highly distinct decidual NK subsets to be resident within first trimester decidua [247].

Analysis of commonly regulated biological pathways, as detailed previously [81], demonstrates that responses to $1,25(\mathrm{OH})_{2} \mathrm{D} 3$ in CK-stimulated $\mathrm{uNK}$ and $\mathrm{pNK}$ diverge significantly from other immune subsets including monocytes, dendritic cells and B cells (Figure 3.4). This may in part reflect different treatment regimens. Here a physiological treatment dose $(10 \mathrm{nM})$ of $1,25(\mathrm{OH})_{2} \mathrm{D} 3$ was utilised, whereas for monocytic THP-1 cells a 10-fold higher treatment dose $(100 \mathrm{nM})$ was applied [217]. Temporal variations may also be a contributory factor. For THP-1 cells, 46 genes were induced/ suppressed after 2.5 h, 288 at $4 \mathrm{~h}$ and 1204 at $24 \mathrm{~h}$. In the current study we assessed mRNA expression in NK cells after $24 \mathrm{~h}$ to maximise potential variations in gene expression; however, both $\mathrm{pNK}$ and $\mathrm{uNK}$ cells may show different patterns of gene regulation by $1,25(\mathrm{OH})_{2} \mathrm{D} 3$ at earlier or later time points. The distinct transcriptional responses to $1,25(\mathrm{OH})_{2} \mathrm{D} 3$ by NK cells may also reflect differential distribution of VDR.

Data reported here provide further evidence for the distinct phenotype of uNK cells relative to circulating pNK. By comparing the gene expression profile of these cells under conditions of immune activation, we confirmed enrichment of distinct functional pathways in uNK such as TGF- $\beta$ signalling (Supplementary Fig. 5C), which has previously been shown to promote uNK development [118]. Our data have also revealed novel features of CK-stimulated uNK, notably enrichment of pathways associated with metabolism, sug- 
gesting a role for uNK cells in immunometabolic activity within the decidua. The lack of $1,25(\mathrm{OH})_{2} \mathrm{D} 3$ response by uNK and pNK contrasts the expression of VDR by both cell types and the enhancement of this receptor in CK-stimulated uNK vs pNK. Given the abundant levels of $1,25(\mathrm{OH})_{2} \mathrm{D} 3$ within decidual tissue [235], and the potent immunomodulatory actions of $1,25(\mathrm{OH})_{2} \mathrm{D} 3$ [47], the lack of response by NK cells is intriguing. Further studies are required to better define the role of the VDR in NK cells. Data in the current study suggest possible cell membrane expression of VDR and this may support non-genomic responses to $1,25(\mathrm{OH})_{2} \mathrm{D} 3$ [102]. In the case of $\mathrm{uNK}$, it is also possible that $1,25(\mathrm{OH})_{2} \mathrm{D} 3$ is able to influence these cells indirectly via other immune cells such as macrophages that have an established transcriptional and functional response to $1,25(\mathrm{OH})_{2} \mathrm{D} 3$. It is now clear that $\mathrm{uNK}$ play a key role in mediating the cross-talk between immune cells within the decidua [247], and vitamin D may therefore play a role in this process. Finally, the data presented in the current study have focused on transcriptional activity but it is also possible that $1,25(\mathrm{OH})_{2} \mathrm{D} 3$ exerts epigenetic effects on NK cells independent of transcription. In recent studies of uNK cells, it has been shown that repeated pregnancies are associated with uNK cells that have a unique transcriptomic and epigenetic signature consistent with trained immune responses [80]. Based on its established epigenetic activity [37], it is tempting to speculate that vitamin D may contribute to this facet of uNK function. 


\section{Chapter 4}

\section{Pathway analysis of transcriptomic data shows immunometabolic effects of vitamin D}

Published in Journal of Molecular Endocrinology in 2018, DOI: 10.1530/JME-17-0186

Amadeo Muñoz García ${ }^{1,2}$, Martina Kutmon ${ }^{1,3}$, Lars M. Eijssen ${ }^{1}$, Martin Hewison ${ }^{2}$, Chris T. Evelo $^{1,3}$, Susan L. Coort ${ }^{1}$

${ }^{1}$ Department of Bioinformatics - BiGCaT, NUTRIM School of Nutrition and Translational Research in Metabolism, P.O. Box 616, Maastricht University, Maastricht, the Netherlands ${ }^{2}$ Institute of Metabolism and Systems Research, The University of Birmingham, Birmingham, B15 2TT, United Kingdom

${ }^{3}$ Maastricht Centre for System Biology (MaCSBio), Maastricht University, Maastricht, the Netherlands 


\subsection{Abstract}

Unbiased genomic screening analyses have highlighted novel immunomodulatory properties of the active form of vitamin D, 1,25-dihydroxyvitamin D $\left(1,25(\mathrm{OH})_{2} \mathrm{D} 3\right)$. However, clearer interpretation of the resulting gene expression data is limited by cell model specificity. The aim of the current study was to provide a broader perspective on common gene regulatory pathways associated with innate immune responses to $1,25(\mathrm{OH})_{2} \mathrm{D} 3$, through systematic re-interrogation of existing gene expression databases from multiple related monocyte models: the THP-1 monocytic cell line (THP-1), monocyte-derived dendritic cells (DCs) and monocytes. Vitamin D receptor (VDR) expression is common to multiple immune cell types, and thus, pathway analysis of gene expression using data from multiple related models provides an inclusive perspective on the immunomodulatory impact of vitamin D. A bioinformatic workflow incorporating pathway analysis using PathVisio and WikiPathways was utilized to compare each set of gene expression data based on pathway-level context. Using this strategy, pathways related to the TCA cycle, oxidative phosphorylation and ATP synthesis and metabolism were shown to be significantly regulated by $1,25(\mathrm{OH})_{2} \mathrm{D} 3$ in each of the repository models (Z-scores 3.52-8.22). Common regulation by $1,25(\mathrm{OH})_{2} \mathrm{D} 3$ was also observed for pathways associated with apoptosis and the regulation of apoptosis (Z-scores 2.49-3.81). In contrast to the primary culture DC and monocyte models, the THP-1 myelomonocytic cell line showed strong regulation of pathways associated with cell proliferation and DNA replication (Z-scores 6.1-12.6). In short, data presented here support a fundamental role for active $1,25(\mathrm{OH})_{2} \mathrm{D} 3$ as a pivotal regulator of immunometabolism.

\subsection{Introduction}

In recent years, studies in vivo and in vitro have shown that vitamin $\mathrm{D}$ is able to influence biological responses that extend far beyond its classical effects on skeletal homeostasis. Prominent among these extra-skeletal effects is the interaction between vitamin D and the immune system, including regulation of both innate and adaptive immune responses [3, 97, 255]. As a consequence of these observations, vitamin D deficiency has been linked to increased risk of bacterial and viral infection [172,137], as well as inflammatory and autoimmune disease $[137,112]$. The ability of supplementary or therapeutic vitamin D to prevent or improve these immune disorders is much less clear and is the subject of randomized placebo control trials currently underway. Crucially, improved understanding of the mechanisms that underpin the immunomodulatory actions of vitamin D has greatly helped to improve the design and outcome of clinical trials. In particular, unbiased analysis of gene responses 
to vitamin D supplementation has uncovered previously unrecognized immune targets for vitamin D [145, 47] that are now key markers of vitamin D function in supplementation trials.

The initial observation linking vitamin $\mathrm{D}$ and immune function was detection of the nuclear vitamin D receptor (VDR) in lymphocytic and myeloid cells [20, 150], indicating that these cells are able to respond to the active form of vitamin $\mathrm{D}, 1,25(\mathrm{OH})_{2} \mathrm{D} 3$, which binds with VDR. Further studies showed that cells from innate immune system such as monocytes/macrophages [128] and dendritic cells (DC) [101] are also able to synthesize $1,25(\mathrm{OH})_{2} \mathrm{D} 3$ from the inactive precursor 25-hydroxyvitamin $\mathrm{D}(25 \mathrm{D})$, the main circulating form of vitamin $\mathrm{D}$. As the principal effect of dietary vitamin $\mathrm{D}$ supplementation is to raise serum levels of $25 \mathrm{D}$, the ability of monocytes and DCs to convert $25 \mathrm{D}$ to $1,25(\mathrm{OH})_{2} \mathrm{D} 3$ provides a localized, autocrine, pathway by which enhanced $25 \mathrm{D}$ can influence both innate and adaptive immunity $[145,112]$. These observations placed monocytes and DCs at the center of the immunomodulatory activity of vitamin D. Synthesis of $1,25(\mathrm{OH})_{2} \mathrm{D} 3$ by these cells has the potential to influence endogenous innate immune cell function in the form of enhanced antibacterial activity [145, 97] and/or modulated antigen presentation [101, 113]. Furthermore, $1,25(\mathrm{OH})_{2} \mathrm{D} 3$ generated by monocytes and/or DCs may also impact adaptive immune function by exerting exogenous effects on T cells or B cells expressing VDR [113]. Despite this, our understanding of the broader impact of vitamin D on cells from the innate immune system such as monocytes, macrophages and DCs remains far from clear.

Recent genome-wide expression studies using a monocyte-derived DC model highlighted the potential role of pathways associated with glucose metabolism, the TCA cycle and oxidative phosphorylation in mediating the effects of $1,25(\mathrm{OH})_{2} \mathrm{D} 3$ in promoting a tolerogenic DC phenotype [72]. The aim of the current study was to provide a broader analysis of monocyte/DC responses to $1,25(\mathrm{OH})_{2} \mathrm{D} 3$ using existing gene expression repositories for multiple models of innate immune function. A meta-analysis of various datasets has been performed using an integrative workflow of open-source bioinformatics tools in order to give a biological context to the genes that are significantly modulated by $1,25(\mathrm{OH})_{2} \mathrm{D} 3$. Finally, a comparison between the immune cell types selected has been applied to highlight the common biological processes that are altered by vitamin $\mathrm{D}$. 


\subsection{Materials and methods}

\subsubsection{Workflow overview}

A workflow was developed that utilizes and integrates gene expression data from public repositories and places them in a biological context. Figure 4.1 illustrates step by step the different tools and methods used in the analysis workflow. The first procedure consisted of open and free modules in ArrayAnalysis to (i) perform a data quality check, (ii) normalize the raw transcriptomic data and (iii) perform a statistical analysis to obtain the parameters that show gene expression changes. The processed datasets were then applied to PathVisio, v. 3.2.4 software [241, 136] in order to perform statistical analyses that highlight the biological processes significantly altered as a consequence of gene expression changes and to visualize them in pathway diagrams from the WikiPathways pathway repository downloaded January 2017 [135]. Comparison of the pathways shown to be significantly altered between the different cell types using PathVisio and WikiPathways was also represented by heat maps diagrams. In another approach, genes shown to be regulated by $1,25(\mathrm{OH})_{2} \mathrm{D} 3$ in all three cell models were used to identify enriched biological processes by gene ontology (GO) analysis and the results were visualized in biological networks using ClueGO, v 2.3.3 [22], an app of Cytoscape 3.4.0 [223] (figure 4.1). This workflow is designed in a user-friendly fashion that does not require programming skills. In order to reproduce this workflow, it is recommended to use the latest versions of designated software. Furthermore, it is also important that gene annotation database and pathways repositories used in the analysis are regularly updated. 


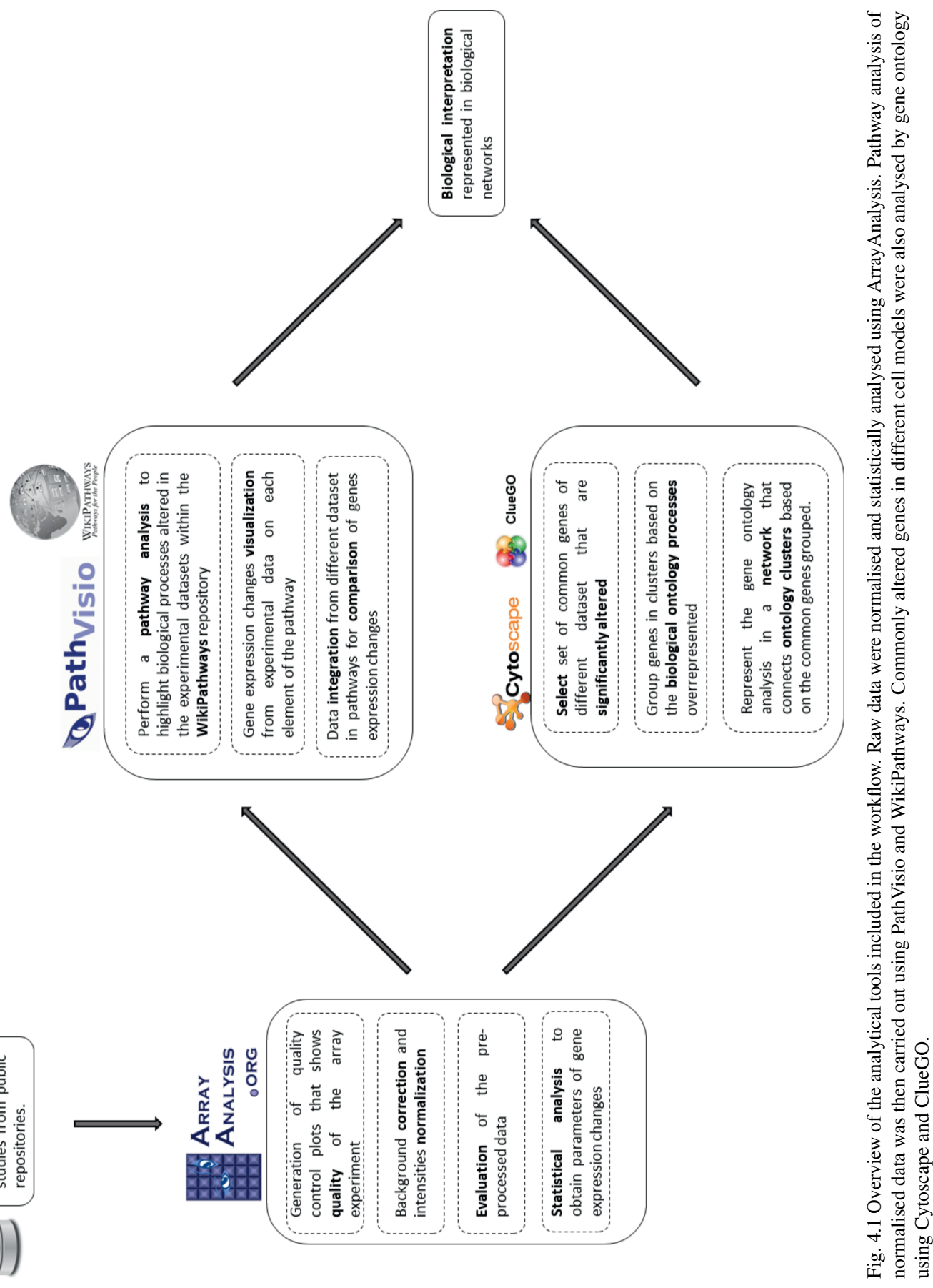




\subsubsection{Transcriptomic datasets}

Publically available transcriptomic data sets for $1,25(\mathrm{OH})_{2} \mathrm{D} 3$-treated monocytes and dendritic cells (DC) were selected from ArrayExpress (https://www.ebi.ac.uk/arrayexpress/, [121]) and Gene Expression Omnibus (GEO, http://ncbi.nlm.nih.gov/geo/, [16]). Selection criteria were based on gene expression studies performed in human cells including monocytic cell lines, monocytes and monocyte-derived DCs treated with $1,25(\mathrm{OH})_{2} \mathrm{D} 3$ : THP-1 cells (GSE52819 [248]), dendritic cells (GSE13762 [231]) and human monocytes (GSE56490 [72]). The selection of the three studies was based on experimental design and quality control analysis of the raw data. In all studies, data were derived from human immune cells treated with $1,25(\mathrm{OH})_{2} \mathrm{D} 3$ or from control (vehicle-treated) cells (table 4.1). In addition to the three cell models outlined previously, preliminary analyses were also carried out using other 1,25(OH) 2 D3-treated cell data repositories: THP-1 cells (GSE60102 ([173]) and human monocytes (GSE46268 [258]). In addition, the data for GSE13762 [231] included both immature and mature DC, and both these models were included in the preliminary analysis. Finally, as a negative control, the preliminary pathway analysis included a $1,25(\mathrm{OH})_{2} \mathrm{D} 3$ treated B cell dataset (GSE22523 [143]). Because of missing raw data, incomplete gene annotation, incompatibility with ArrayAnalysis and non-monocyte origin, some data from the preliminary analysis were not included in the main analysis of this study, but relevant findings related to the data were addressed in the 'Results' section.

\subsubsection{Pre-processing raw data and statistical data analysis in Array- Analysis}

Unprocessed transcriptomics data were collected from the repositories and processed using the online workflow of ArrayAnalysis performed in January 2017 (https://www.arrayanalysis. org [64]) to obtain quality control reports, normalized data and perform statistical analysis. The workflow uses an R script as a core module with functions from several Bioconductor libraries. Quality control showed that there was no need to exclude any samples in the three selected transcriptomic datasets (data not shown). Depending on the microarray technology used, the normalization algorithm was selected. RMA method was used to normalize GSE52819 [248] and GSE56490 [72]. GC-RMA method was applied to GSE13762 [231]. Statistical analysis of the normalized data to determine genes that are significantly altered (up/downregulated) was carried out with a second module from ArrayAnalysis including the moderated t-test from the R/Bioconductor package limma [205]. The output is a table with the expression parameters for each gene showing fold-change (FC), in a log scale after $1,25(\mathrm{OH})_{2} \mathrm{D} 3$ treatment, and the significance of changes by the Benjamini-Hochberg adjusted 
$\mathrm{P}$ value. Based on statistical gene expression parameters, the significantly up/downregulated genes were identified using the criteria: absolute $\log 2 \mathrm{FC}>0.26$ and adjusted $\mathrm{P}$ value $<0.05$. The average expression (AE) was used to filter out lowly expressed genes, and the cut-off was specific for each dataset based on the density plot of the intensities after normalization: THP-1 AE > 4.25; immature DC AE >3.86 and monocytes AE >9.5 table 4.1.

\subsubsection{Pathway statistics and analysis in PathVisio}

PathVisio v. 3.2.4 [241, 136], an open-source pathway creation and analysis tool, was used to contextualize significantly altered genes, and altered biological processes were visualized using the biological pathway repository WikiPathways [135]. Utilizing the BridgeDb identifier mapping feature released on 18 October 2016 (http://www.bridgedb.org/) [242], PathVisio recognizes genes (probe) identifiers from the most used databases and microarray platforms such as NCBI and Affymetrix. This identifier mapping database (Homo sapiens Derby Ensembl 85 gene database) enables linking of statistical values from analyzed data to the corresponding gene boxes in the pathway diagrams of WikiPathways. In the analysis, the curated human pathways collection (released April 2017, http://data. wikipathways.org/20170410/gpml/) of WikiPathways was used. For pathway analysis, a criterion is chosen to select genes that are significantly altered within each dataset based on the expression difference parameter of FC (on $\log 2$ scale) and the significance of that change is represented as the adjusted $\mathrm{P}$ value $(<0.05)$. PathVisio performs an overrepresentation analysis taking into account all genes measured $(\mathrm{N})$, genes that satisfy the criterion $(\mathrm{R})$, genes measured in the experiment that are present in the pathway (n) and genes in the pathway measured in the experiment that satisfy the criteria (r):

$$
\text { ZScore }=\frac{r-n \frac{R}{N}}{\sqrt{n\left(\frac{R}{N}\right)\left(1-\frac{R}{N}\right)\left(1-\frac{n-1}{N-1}\right)}}
$$

The Z-score is used to measure how significantly a subset of genes is altered in a certain pathway compared to the complete dataset. In our analysis, biological pathways that have a Zscore equal to or above 1.96 are considered significantly altered. The function heatmap. 2 from the R library gplots (v.3.0.1) was used to create a heatmap that compared and hierarchically clustered pathways for each of the datasets based on Z-scores. 
76 Pathway analysis of transcriptomic data shows immunometabolic effects of vitamin D

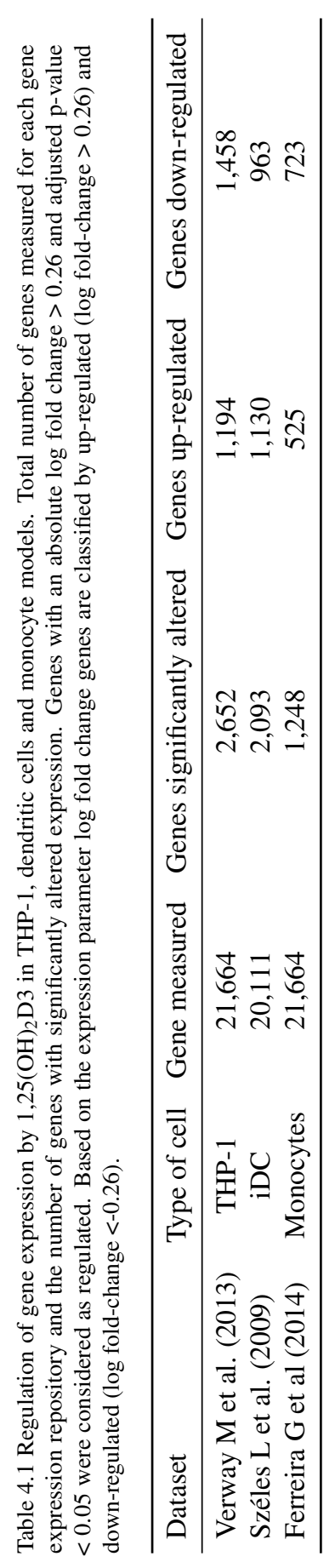




\subsubsection{GO analysis with ClueGO in Cytoscape}

$\mathrm{GO}$ analysis of the common significantly altered genes (absolute $\log 2 \mathrm{FC}>0.26$ and adjusted $\mathrm{P}$ value $<0.05$ ) in the three types of immune cells was performed to identify and visualize which GO biological process terms were significantly overrepresented. Within the commonly used open-source network analysis tool Cytoscape, v. 3.4 [223], the ClueGO app, v 2.3.3 performs GO analysis to group a set of genes in GO terms minimizing redundancy and display their relationship in a network. Connections between the GO nodes containing a common subset of genes were calculated with kappa statistics based on the correlated genes that are grouped.

\subsection{Results}

\subsubsection{Differentially expressed genes in $1,25(\mathrm{OH})_{2}$ D3-treated THP1 cells, dendritic cells and monocytes}

A preliminary pathway analysis of multiple THP-1, monocyte and DC datasets revealed common regulation of several key cell pathways by $1,25(\mathrm{OH})_{2} \mathrm{D} 3$ in cells from the myeloid lineage: electron transport, oxidative phosphorylation, the TCA cycle, glycolysis and gluconeogenesis and apoptosis. By contrast, the non-myeloid B cell gene expression dataset showed no similarity with the myeloid effects of $1,25(\mathrm{OH})_{2} \mathrm{D} 3$, underlining the lineagespecific effects of $1,25(\mathrm{OH})_{2} \mathrm{D} 3$ (figure 4.2). From this initial pathway analysis, it was also interesting to note that $1,25(\mathrm{OH})_{2} \mathrm{D} 3$ regulation of pathways associated with the cell cycle and cell proliferation were only observed in the myeloid leukemic cell line THP-1 and not the other non-neoplastic primary cell models used in the study (figure 4.2). Because of incomplete raw data or gene annotation, or incompatibility with ArrayAnalysis, further, more detailed, pathway analysis was restricted to raw datasets for GSE52819, GSE13762 and GSE56490. 


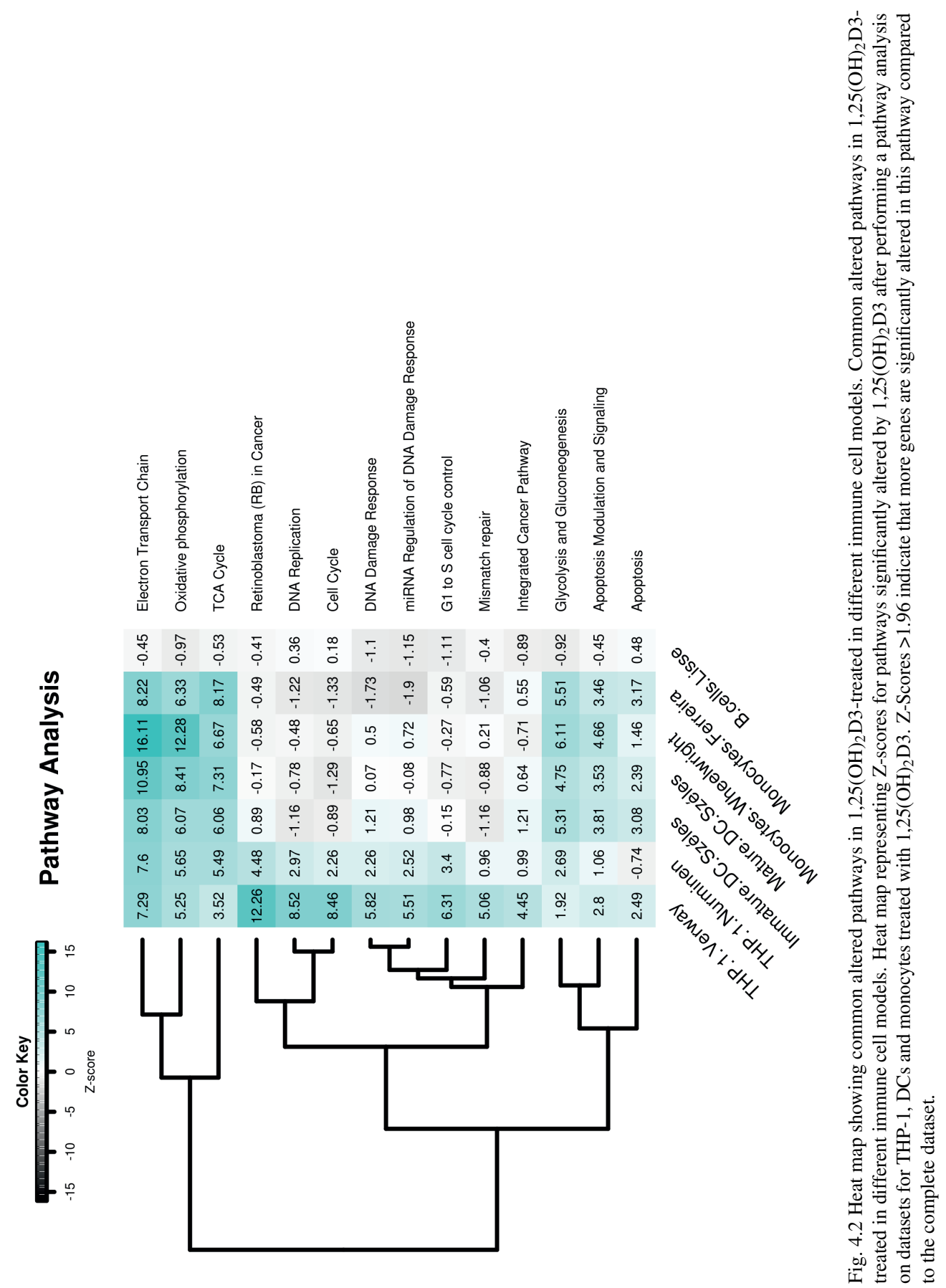




\subsubsection{Pathway analysis reveals altered biological processes based on molecular changes in $1,25(\mathrm{OH})_{2} \mathrm{D} 3$-treated immune cells}

Pathway overrepresentation analysis performed on immune cell datasets GSE52819, GSE13762 and GSE56490 generated a list of biological processes regulated by $1,25(\mathrm{OH})_{2} \mathrm{D} 3$ in each of the three different innate immune cell models. For each cell type, the significantly altered pathways were selected, and their Z-scores plotted and clustered in a heatmap (figure 4.2) showing Z-score comparisons for major affected pathways between the three cell models. Data indicate that $1,25(\mathrm{OH})_{2} \mathrm{D} 3$-regulated pathways were either cell specific or common to all three models. Pathways related to DNA replication, the cell cycle and cancer (retinoblastoma) showed high $\mathrm{Z}$-scores and were more strongly regulated by $1,25(\mathrm{OH})_{2} \mathrm{D} 3$ in THP-1 cells, consistent with the proliferative leukemic nature of this cell line. By contrast, primary cells (DCs and monocytes) showed stronger $1,25(\mathrm{OH})_{2} \mathrm{D} 3$ regulation of pathways associated with glycolysis/gluconeogenesis and the apoptosis-related network due to altered Notch3 in ovarian cancer pathway relative to THP-1 cells, albeit at lower Z-scores than observed for THP-1-specific pathways. Pathways associated with apoptosis and apoptosis modulation and signaling also showed lower Z-scores, but were equally induced by $1,25(\mathrm{OH})_{2} \mathrm{D} 3$ in THP-1 cells, DCs and monocytes. The highest Z-score for 1,25(OH $)_{2} \mathrm{D} 3$-regulated pathways common to all three cell models was for pathways related to the TCA cycle, oxidative phosphorylation and the electron transport chain.

\subsubsection{GO analysis shows metabolic, immunological and apoptotic pro- cesses altered by $1,25(\mathrm{OH})_{2} \mathrm{D} 3$ in THP-1 cells, dendritic cells and monocytes}

ArrayAnalysis processing of GSE52819, GSE13762 and GSE56490 datasets was carried out to define the number of genes measured and number of genes significantly regulated by $1,25(\mathrm{OH})_{2} \mathrm{D} 3$ in each of the three cell models (table 4.1). Further analysis of these data showed that a total of 230 genes were significantly regulated by $1,25(\mathrm{OH})_{2} \mathrm{D} 3$ in all three cell models (figure 4.3), and these genes were selected for subsequent GO analysis.

GO analysis using the list of 230 commonly altered genes was carried out using ClueGO in Cytoscape to display networks of overrepresented biological processes associated with this list. A total of 39 groups of GO terms resulted in the identification of three broad sub-networks common to each of the cell models (figure 4.4, figure 4.5 and figure 4.6). 


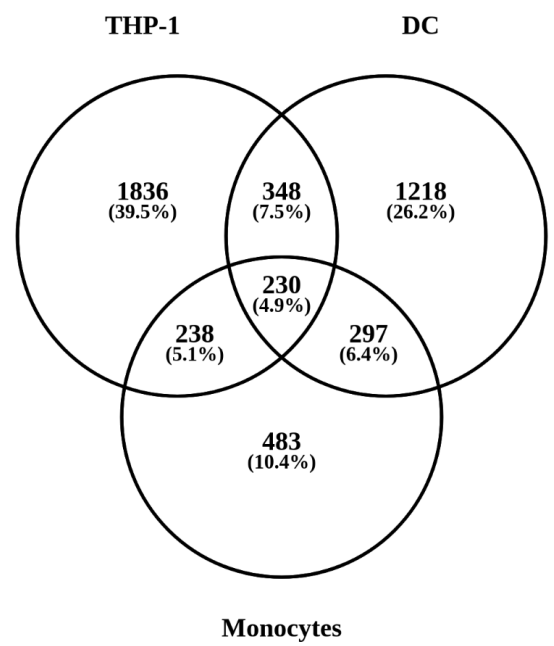

Fig. 4.3 Venn diagram showing numbers of common and cell-specific genes regulated by $1,25(\mathrm{OH})_{2} \mathrm{D} 3$. $1,25(\mathrm{OH})_{2} \mathrm{D} 3$-regulated genes in THP-1 cells, DCs and monocytes. Venn diagrams showing numbers of common and cell-specific genes regulated by $1,25(\mathrm{OH})_{2} \mathrm{D} 3$ in THP- 1 cells, DCs and monocytes. Genes with an absolute $\log 2$ fold-change $>0.26$ and adjusted $\mathrm{P}$ value $<0.05$ were considered as regulated.

The first of these networks was associated with bioenergetic GO terms including the electron transport chain (GO:22904), oxidation-regulation process (GO:55114), oxidative phosphorylation (GO:6119), tricarboxylic acid cycle (GO:6099) and canonical glycolysis (GO:61621) (figure 4.4). A second sub-network was based on immunological GO terms such as responses to molecules of bacterial origin (GO:2237), cytokine production (GO:1816), inflammatory response (GO:6954) and myeloid cell differentiation (GO:30099) (figure 4.5). The final network identified groups of apoptotic processes such as intrinsic apoptotic signaling (GO:97193) and negative regulation of apoptotic signaling (GO:2001234) (figure 4.6).

\subsubsection{Visualization of $1,25(\mathrm{OH})_{2} \mathrm{D} 3$-induced changes in gene expres- sion in THP-1 cells, DCs and monocytes on altered metabolic pathways}

Pathways related to ATP metabolism showed high Z-scores (>1.96), indicating strong regulation by $1,25(\mathrm{OH})_{2} \mathrm{D} 3$ (figure 4.2). To investigate the significant changes in gene expression in more depth, the effect of $1,25(\mathrm{OH})_{2} \mathrm{D} 3$ on gene expression was visualized on the altered metabolic pathways discovered with pathway analysis, i.e., electron transport chain, oxidative phoshorylation and TCA cycle and glycolysis. 


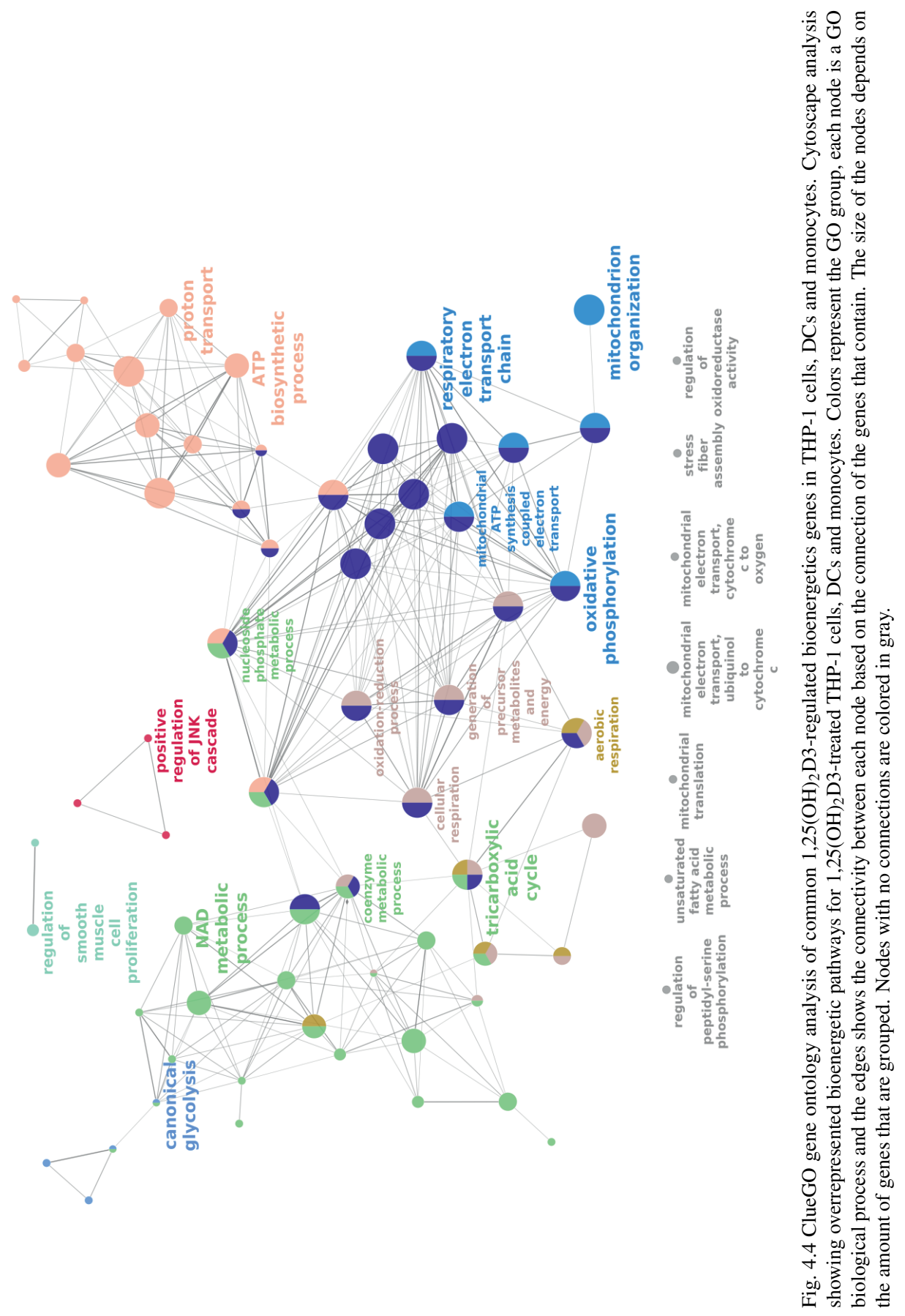


82 Pathway analysis of transcriptomic data shows immunometabolic effects of vitamin D

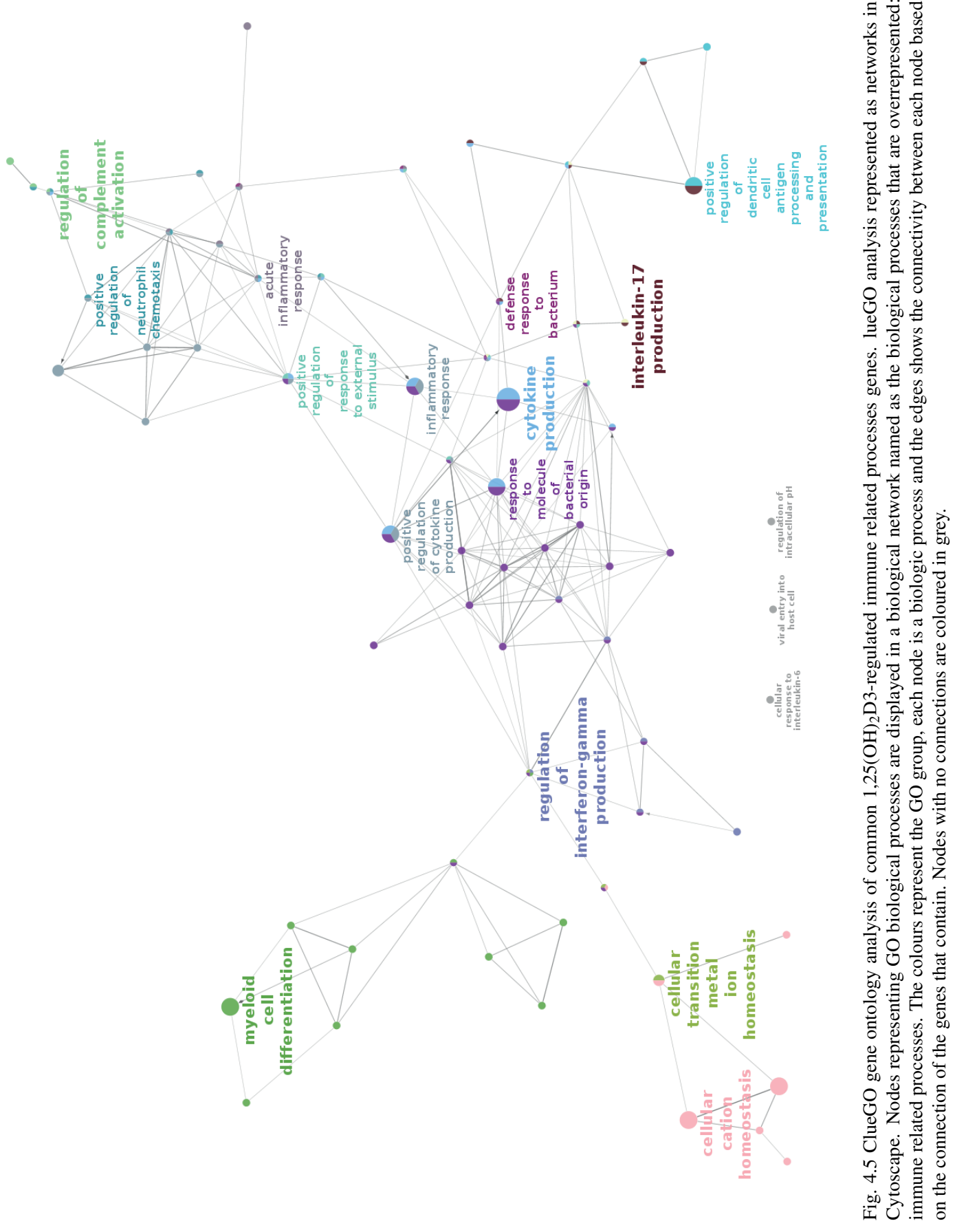




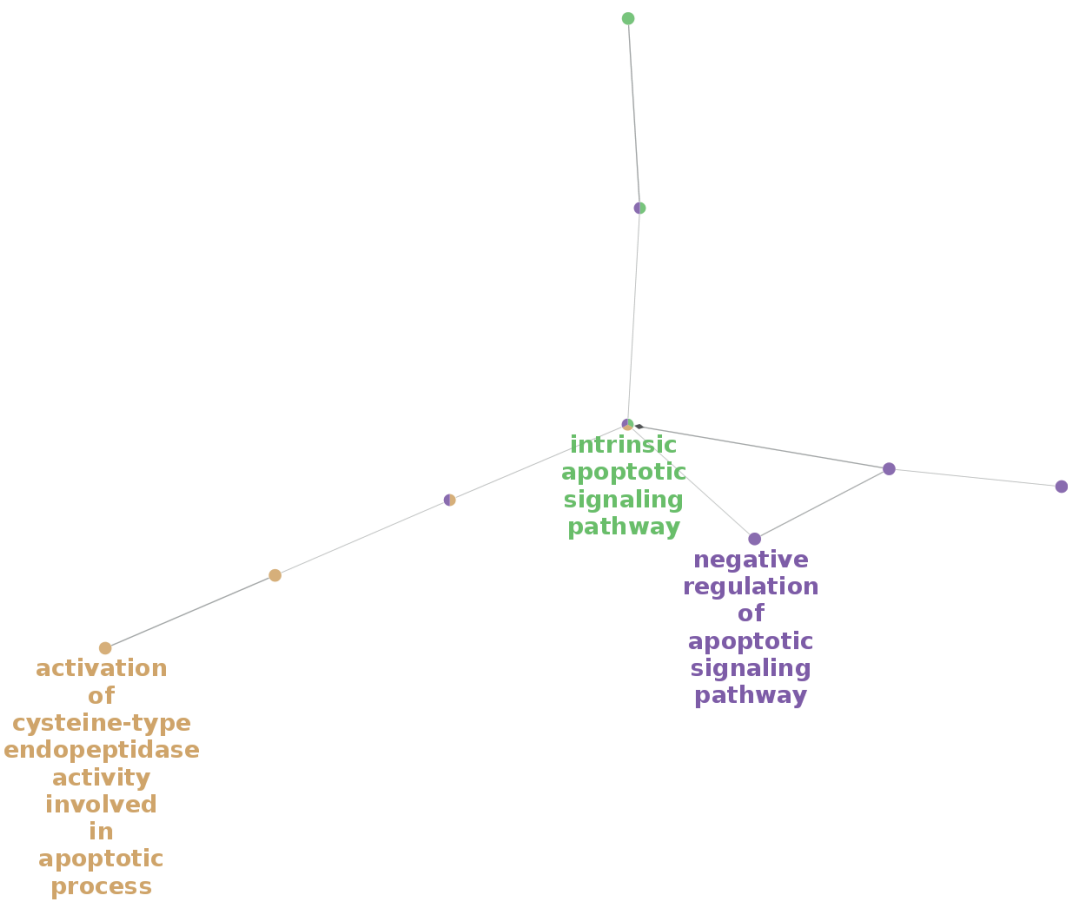

Fig. 4.6 ClueGO gene ontology analysis of common 1,25(OH) 2 D3-regulated apoptosis related processes genes. ClueGO analysis represented as networks in Cytoscape. Nodes representing GO biological processes are displayed in a biological network named as the biological processes that are overrepresented: apoptosis related processes. The colours represent the GO group, each node is a biologic process and the edges shows the connectivity between each node based on the connection of the genes that contain. Nodes with no connections are coloured in grey. 


\subsection{5 $1,25(\mathrm{OH})_{2} \mathrm{D} 3$ and the electron transport chain}

In the human electron transport chain pathway (http://wikipathways.org/index.php/Pathway: WP111), significantly upregulated or downregulated genes (adj. P value $<0.05$ ) in the three immune cells treated with $1,25(\mathrm{OH})_{2} \mathrm{D} 3$ compared to untreated cells were collectively visualized. Overall, the electron transport chain was activated by $1,25(\mathrm{OH})_{2} \mathrm{D} 3$ in the three immune cells. Biological data in figure 4.7 shows in all three cell types a significant increase in expression by $1,25(\mathrm{OH})_{2} \mathrm{D} 3$ of genes associated with electron transport chain complexes I (NDUFA2, NDUFA3, NDUFA8, NDUFB9, NDUFA10, NDUFB5, NDUFAB1, NDUFS3, NDUFV1, NDUFS1), complex II (SDHB), complex III (UQRC1-2), complex IV (COX411, COX5A) and complex V (ATP5A1, ATP5B, ATP5C1, ATP5G1, ATP5L). Interestingly, the gene expression of the uncoupling protein 2 (UCP2) is significantly (adj. P value <0.05) downregulated in THP-1 cells and monocytes by $1,25(\mathrm{OH}) 2 \mathrm{D}$, but unchanged in dendritic cells.

\subsubsection{1,25 $(\mathrm{OH})_{2} \mathrm{D3}$ and oxidative phosphorylation}

In the oxidative phosphorylation pathway (http://wikipathways.org/index.php/Pathway:WP623) all significantly changed genes were upregulated, demonstrating that this process is activated by $1,25(\mathrm{OH})_{2} \mathrm{D} 3$ in THP-1 cells, DCs and monocytes. Biological data in figure 4.9 show that specifically genes related to ATP synthase (ATPG3, ATP5G1, ATP5B, ATP5G1, ATP5A1, ATP5L) and nicotinamide nucleotide transhydrogenase (NDUFA3, NDUFS3, NDUFV1, NDUFA10, NDUFA8, NUDFB9 NDUFS1, NDUFB5, NDUFAB1, NDUFA2) are significantly (adj. P value $<0.05$ ) upregulated by $1,25(\mathrm{OH})_{2} \mathrm{D} 3$ in each of the cell models.

\subsection{7 $1,25(\mathrm{OH})_{2} \mathrm{D3}$ and the TCA cycle and glycolysis}

In the TCA cycle (http://wikipathways.org/index.php/Pathway:WP78), the process that produces energy by oxidation of acetyl- $\mathrm{CoA}, 1,25(\mathrm{OH})_{2} \mathrm{D} 3$ upregulates genes involved in the conversion of acetyl-CoA in carbohydrate and chemical energy. $1,25(\mathrm{OH})_{2} \mathrm{D} 3$ increased the expression of ACO2, IDH3, IDH4B, DLD, SUCLG1, SDHB and MDH2 and only the expression of IDH2, which functions in the opposite direction of the cycle, was significantly (adj. P value <0.05) downregulated (figure 4.10). Glucose metabolism was also affected by $1,25(\mathrm{OH})_{2} \mathrm{D} 3$, with enzymes such as HK2, PFKM and FBP1 being significantly (adj. P value $<0.05$ ) upregulated after treatment (figure 4.8). 

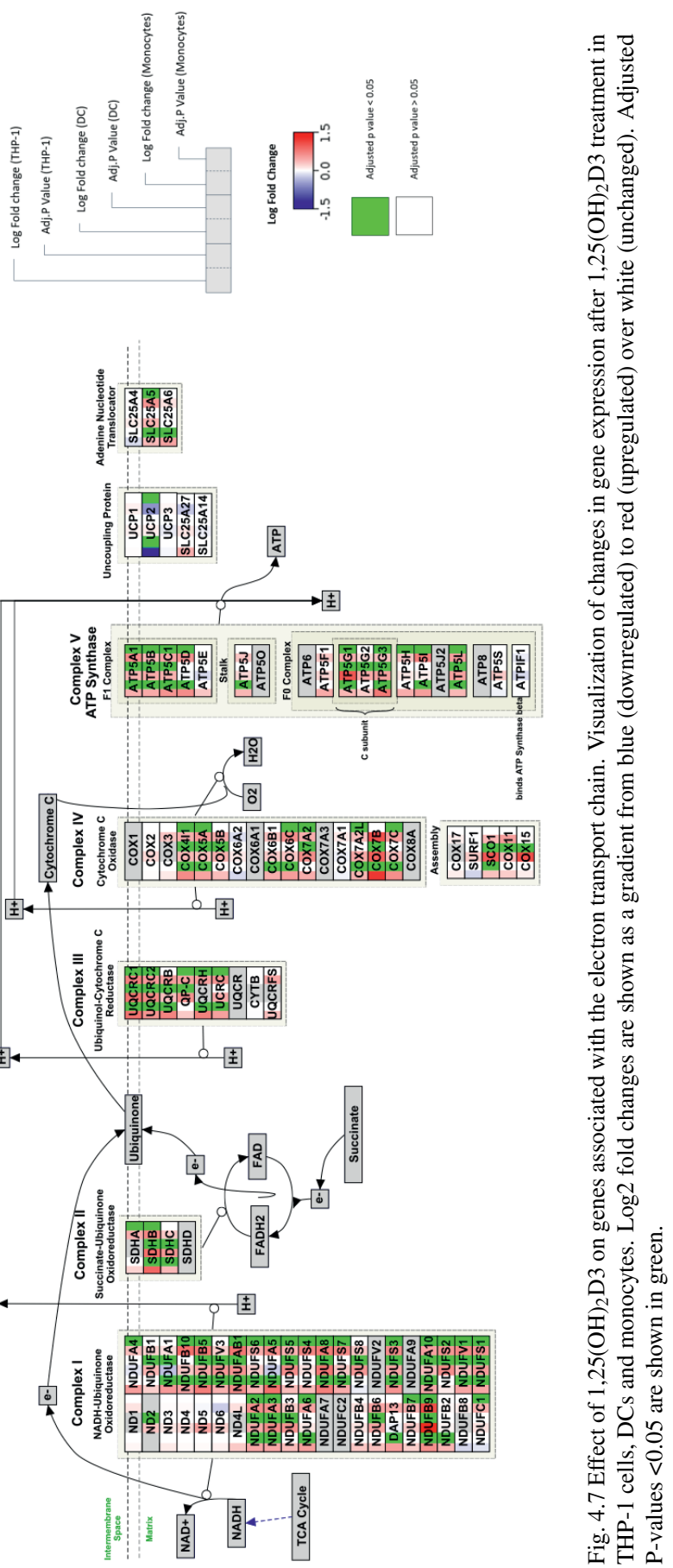


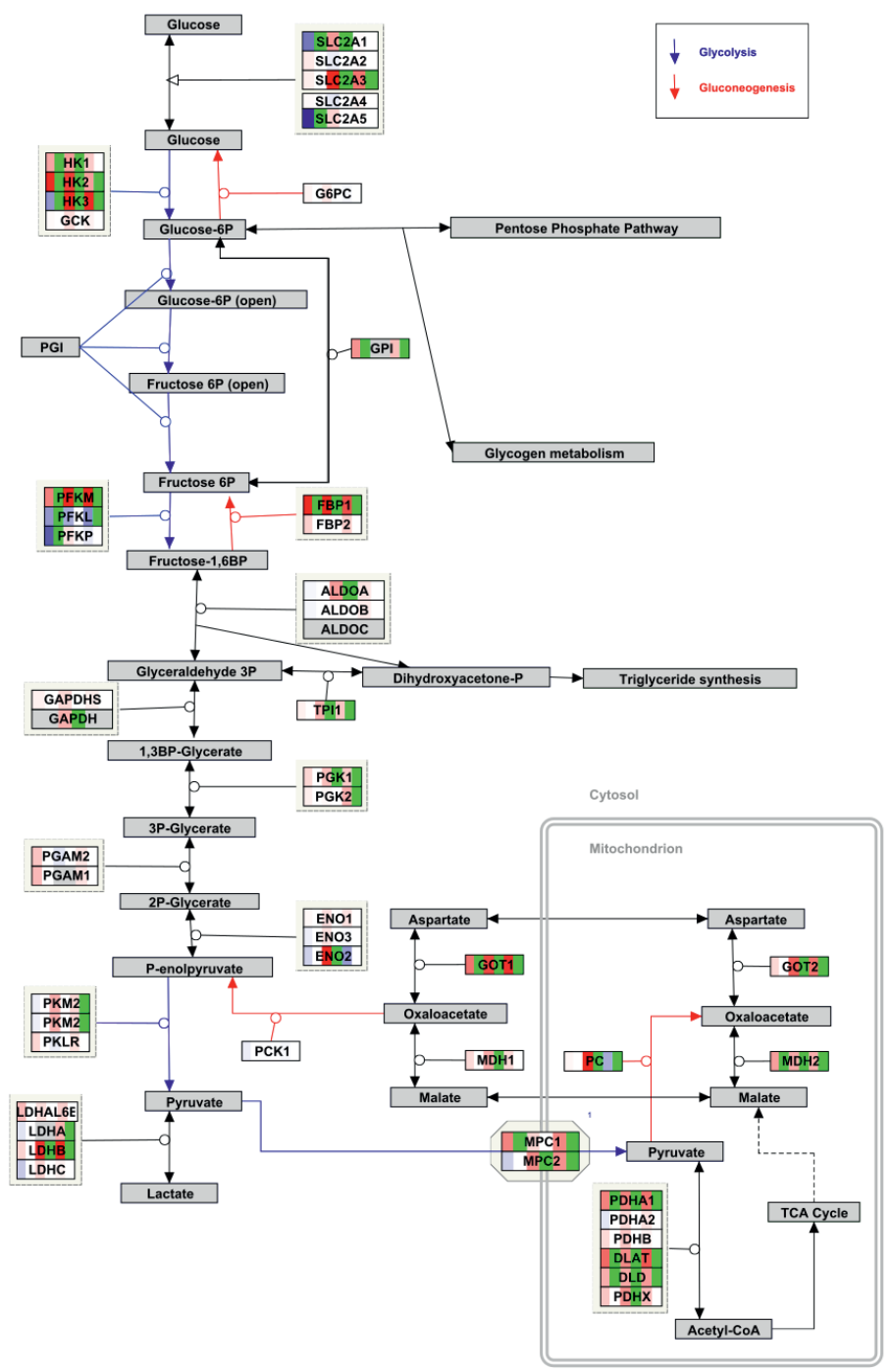

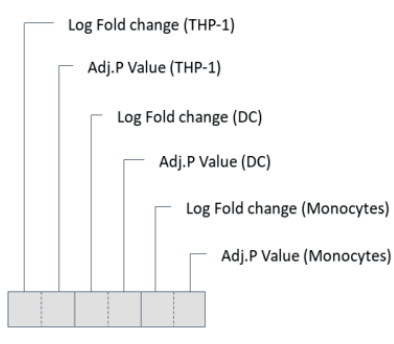

Log Fold Change

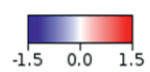

Adjusted p value $<0.05$

Adjusted p value $>0.05$

Fig. 4.8 Effect of $1,25(\mathrm{OH})_{2} \mathrm{D} 3$ on genes associated with the glycolysis. Visualization of changes in gene expression after $1,25(\mathrm{OH})_{2} \mathrm{D} 3$ treatment in THP-1 cells, DCs and monocytes. Log2 fold changes are shown as a gradient from blue (downregulated) to red (upregulated) over white (unchanged). Adjusted P-values $<0.05$ are shown in green. 

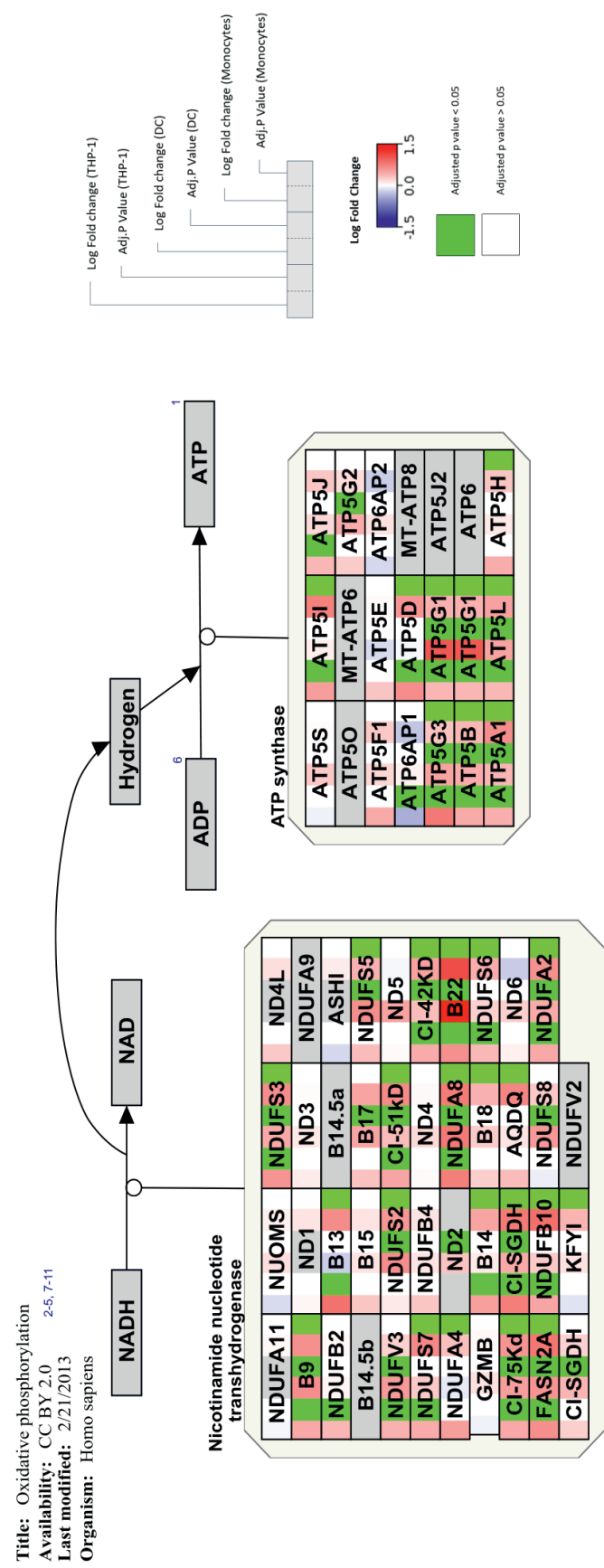

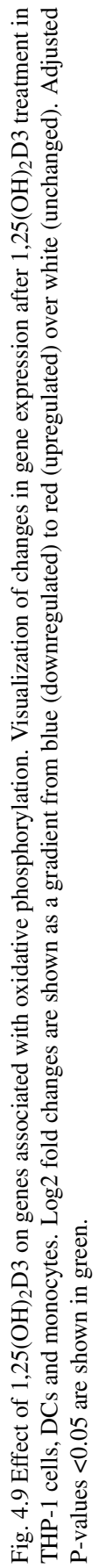



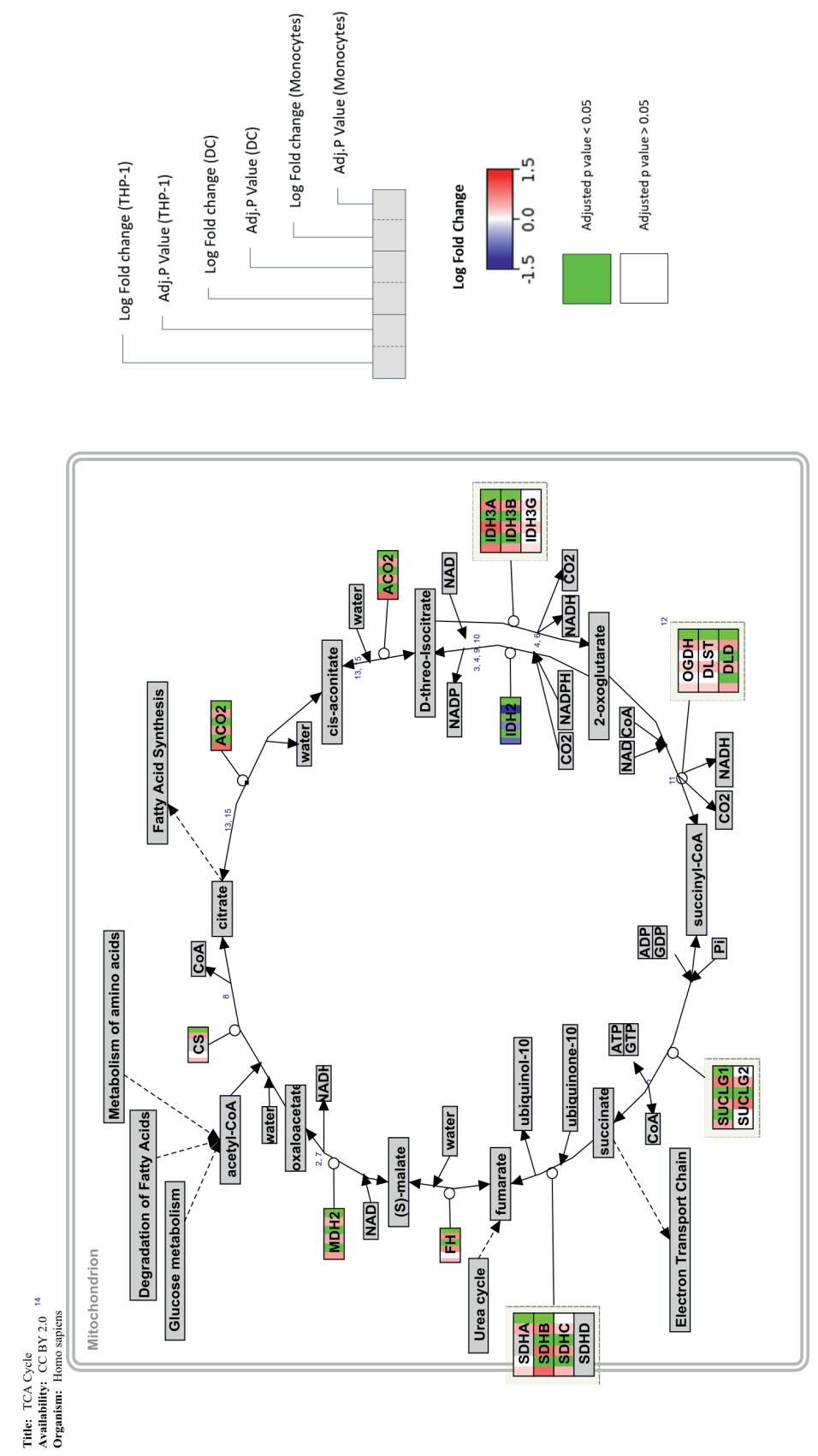

永营

$\Xi$

吾总

จ

I

응

곤

巳્ฮ

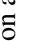

के

ปิํํㄹ

$\Xi$ D

要

층

tั

.ำ

宽

軋

$5 \cong$

옹

论 营

范

$\cong$ ต

3

.

o

जै

樆 总

흥ㅇㅇ

กิ के

동오

는

응

总

ㅇํำ

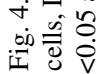




\subsection{8 $1,25(\mathrm{OH})_{2} \mathrm{D} 3$ and cell proliferation}

Analysis of both THP-1 cell datasets revealed that $1,25(\mathrm{OH})_{2} \mathrm{D} 3$ had a significant effect on genes associated with the cell cycle (https://www.wikipathways.org/instance/WP179_r93002) (figure 4.11). This included suppression of complexes involved in phase transitions and checkpoint signaling such as BUB1/BUB3, p34cdc2/cyclin B, Chk1/Chk2 and cell cyclindependent kinases (CDKs) such as CDK1, CDK2 and associated cyclins A1, A2, B1, B2 and E1, E2. THP-1 cells treated with $1,25(\mathrm{OH})_{2} \mathrm{D} 3$ also showed decreased expression of genes associated with DNA replication (https://www.wikipathways.org/instance/WP466_ r94886) (figure 4.12). This included suppression of subunits of the maintenance protein complex (MCM), needed to initiate and elongate the replication fork in the DNA replication. $1,25(\mathrm{OH})_{2} \mathrm{D} 3$ also suppressed expression of genes that participate in progression of the cell cycle such as components of the assembly of the pre-replicative complex. This includes subunits of the component of the origin recognition complex (ORC): ORCL 1, 3 and 6. Genes that participate in the assembly of pre-replicative DNA complexes were also downregulated by $1,25(\mathrm{OH})_{2} \mathrm{D} 3$. These include CDC6 and CDT1/GMNN complex. Finally, components of the DNA polymerase (POLa2, POLa, POLe2), primase (PRIM1, PRIM2) and subunits of the replication factor $\mathrm{C}(\mathrm{RFC} 1-5)$ were also downregulated by $1,25(\mathrm{OH})_{2} \mathrm{D} 3$ in THP-1 cells (figure 4.12). In contrast to effects in leukemic THP-1 cells, pathways associated with cell proliferation and cell cycling were unaffected by $1,25(\mathrm{OH})_{2} \mathrm{D} 3$ in primary cultures of monocytes and dendritic cells (figure 4.2).

\subsection{9 $1,25(\mathrm{OH})_{2} \mathrm{D3}$ and apoptosis}

In the GO analysis, a network that relates apoptosis GO terms revealed three GO core groups: (1) negative regulation of apoptotic signaling; (2) intrinsic apoptotic signaling; (3) regulation of intrinsic apoptotic signaling pathway (figure 4.6). These groups include genes that were upregulated by $1,25(\mathrm{OH})_{2} \mathrm{D} 3$ in all three cells types such as: NDUFS3, TNFSF10, TRAP1, YBX3, NCK2, CEBPB, FXN, CYCS and NCK2. Conversely, CD74, DAPK1, ITGAV and FNIP2 were downregulated by $1,25(\mathrm{OH})_{2} \mathrm{D} 3$. Biological data in figure 4.13 and figure 4.14 show significant effects of $1,25(\mathrm{OH})_{2} \mathrm{D} 3$ on pathways associated with apoptosis. The receptor TNFTSF10D that participates negatively in apoptosis is upregulated by $1,25(\mathrm{OH})_{2} \mathrm{D} 3$ in all three cell types, while the family of apoptotic caspases $(2,3,4,6$ and 7$)$ and the apoptotic peptidase-activating factor 1 (APAF1) are suppressed. Finally, it is interesting to note that JAK2 and MAP3K5 are downregulated by $1,25(\mathrm{OH})_{2} \mathrm{D} 3$ in THP-1 cells but upregulated in DCs and monocytes. 


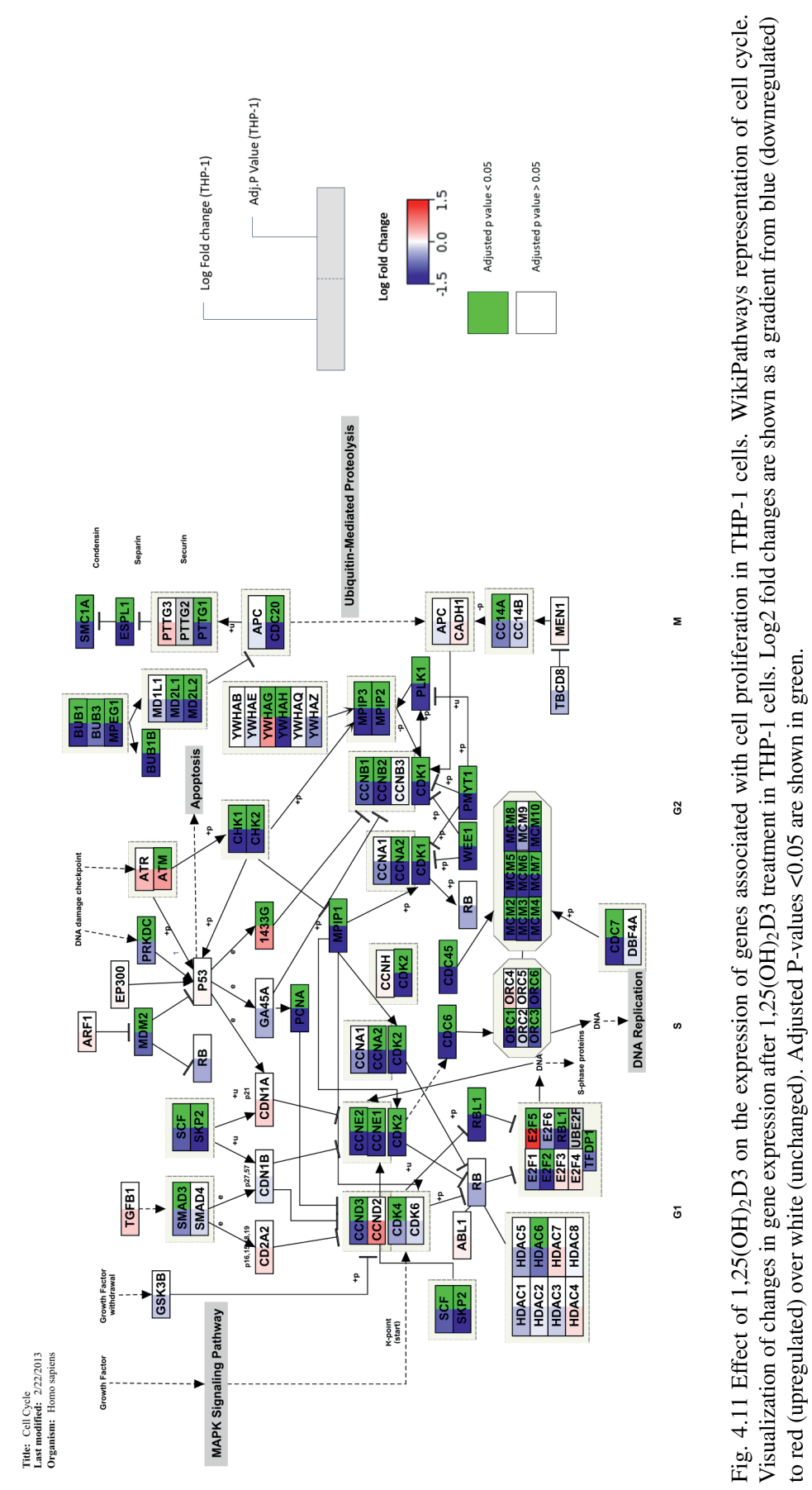




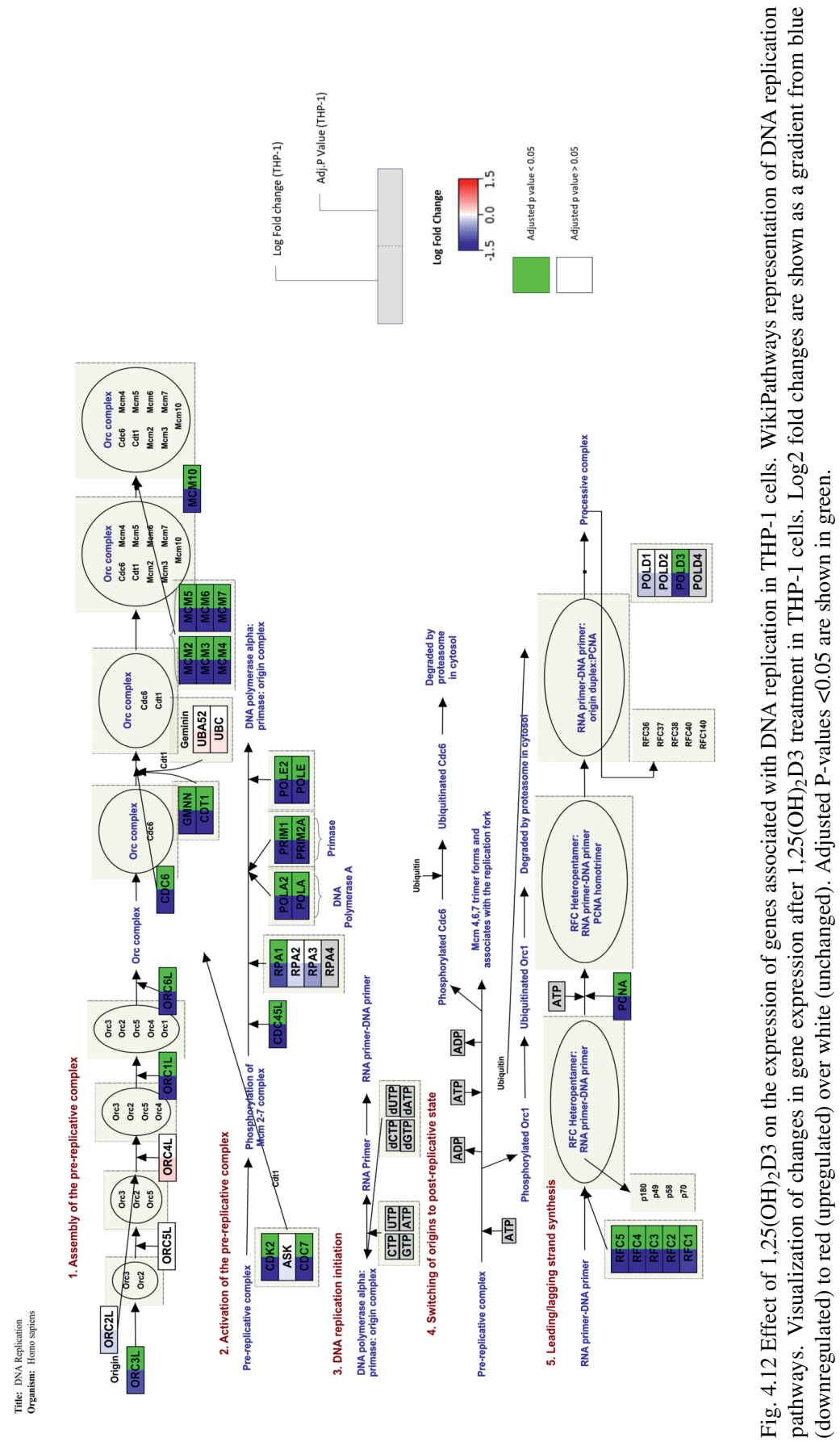




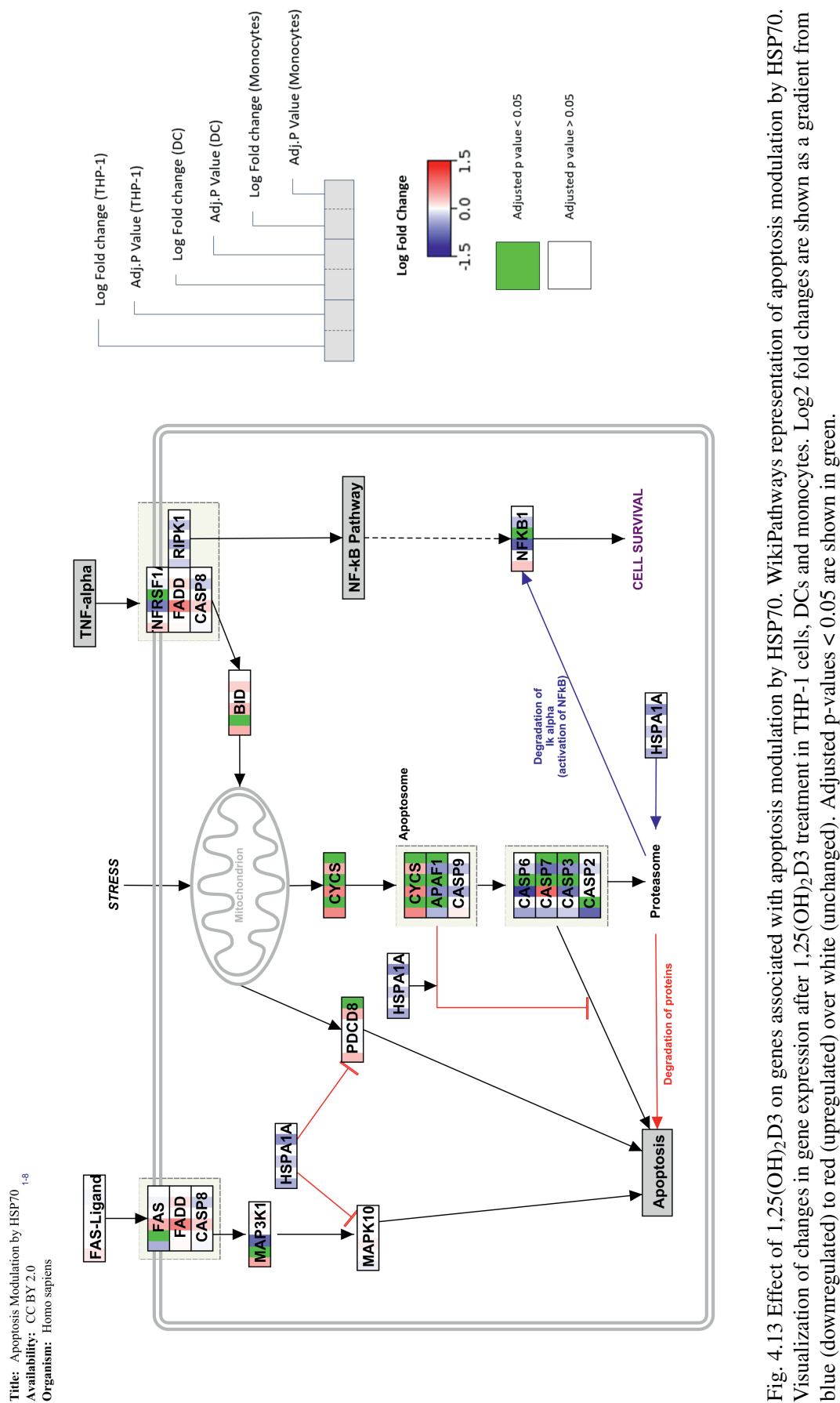

$\stackrel{\circ}{\hat{2}}$

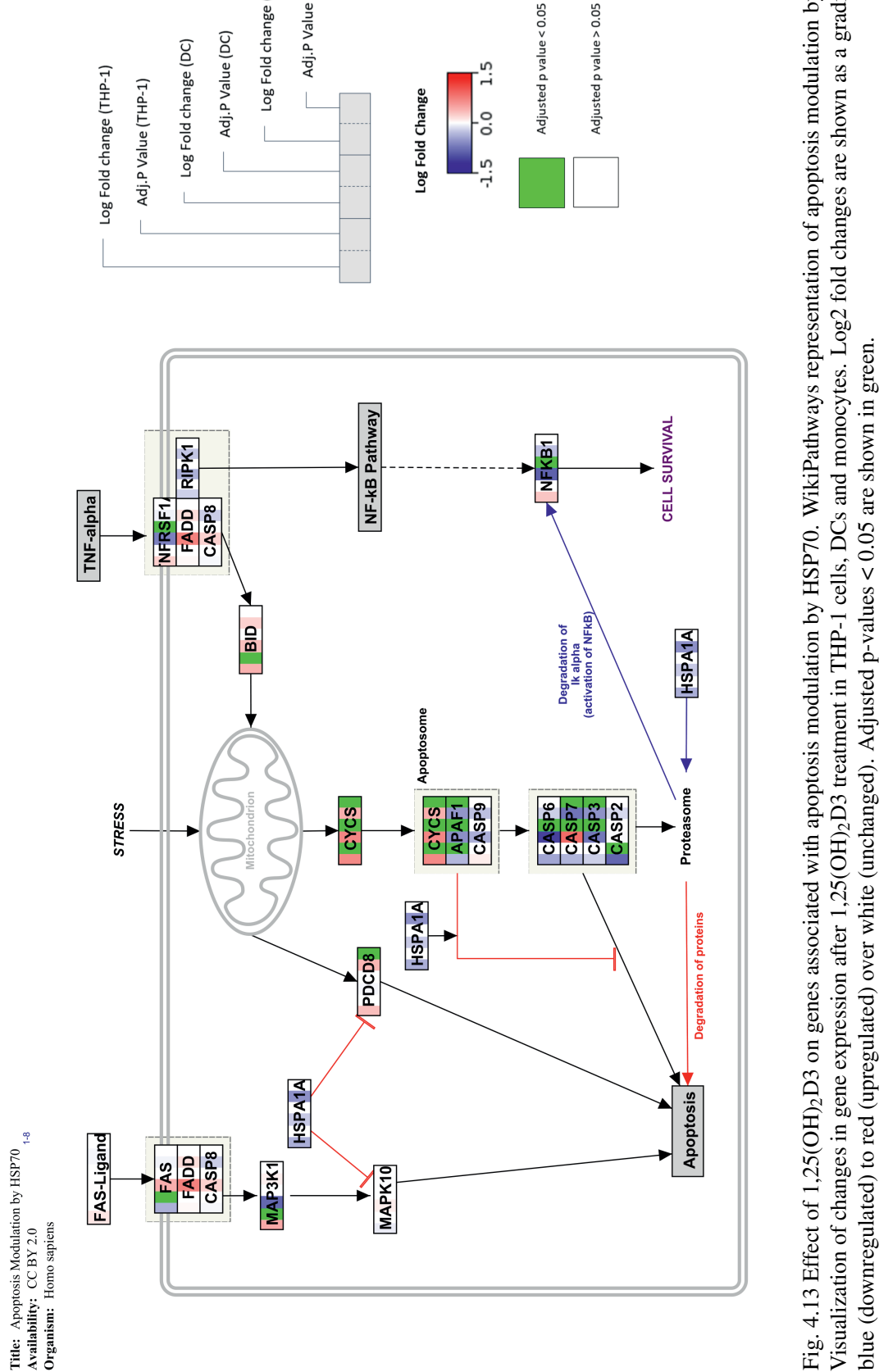




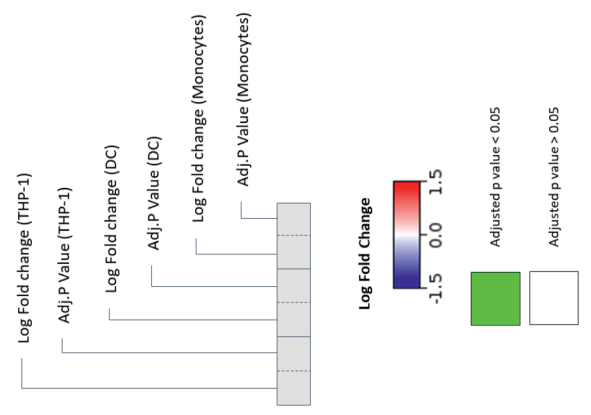

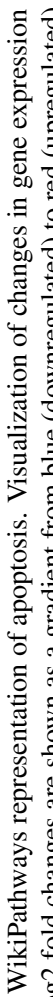
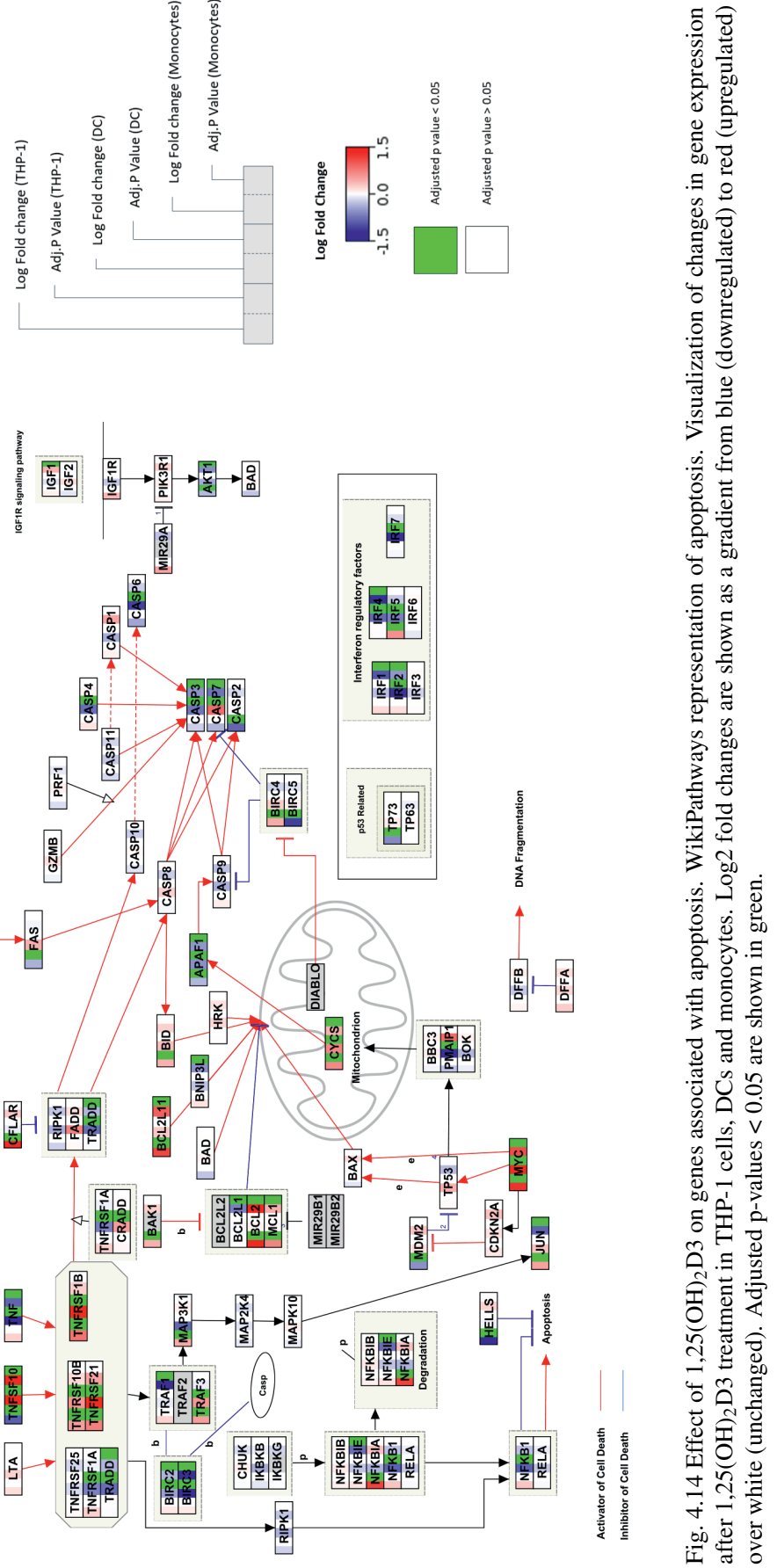

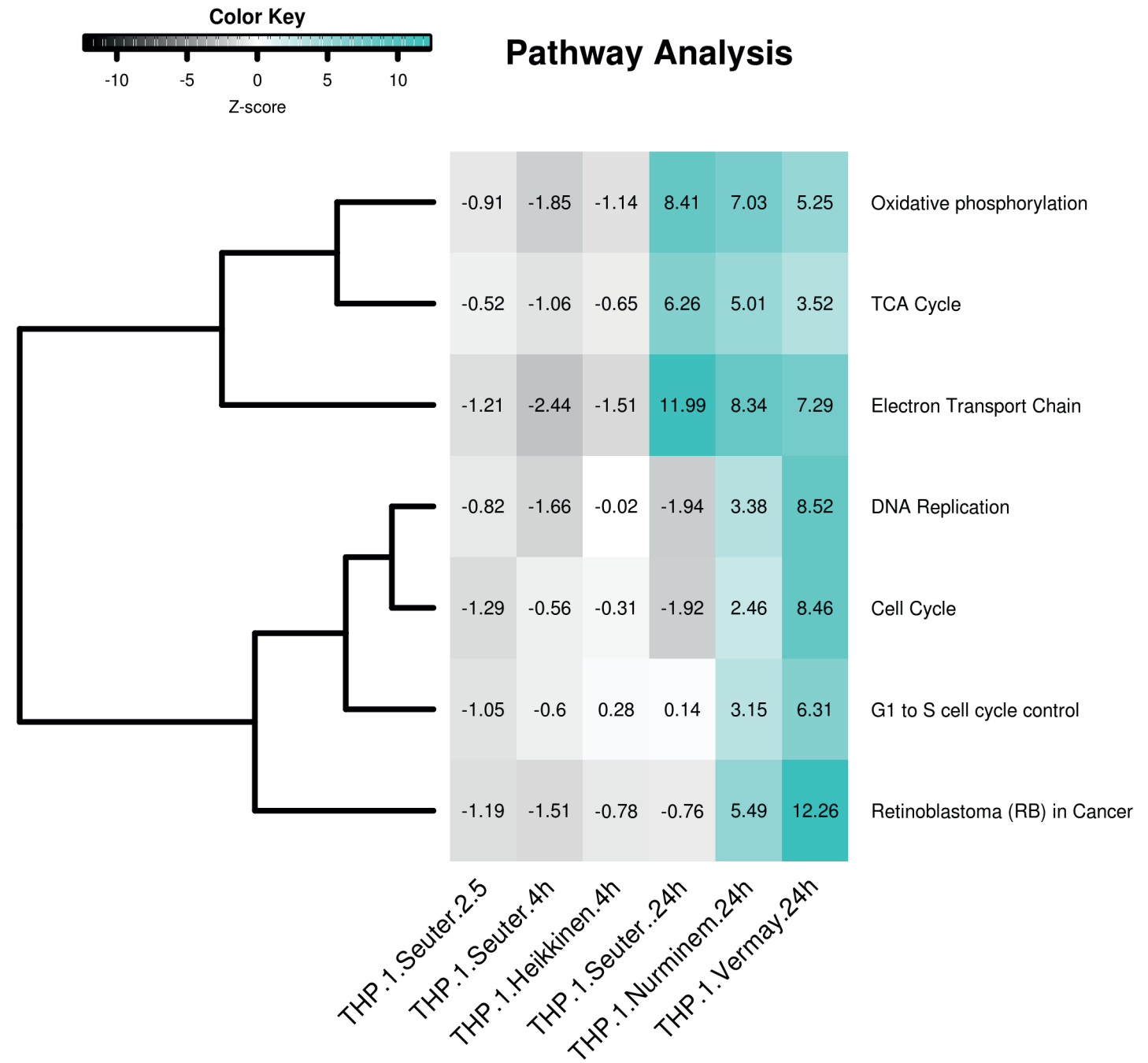

Fig. 4.15 Heat map showing temporal effect of $1,25(\mathrm{OH})_{2} \mathrm{D} 3$ treatment in THP-1 cells. Temporal effect of $1,25(\mathrm{OH})_{2} \mathrm{D} 3$ treatment in THP-1 cells To evaluate temporal effects of $1,25(\mathrm{OH})_{2} \mathrm{D} 3$ comparison of pathway analysis was carried out for THP-1 cell array analyses at 24 hours (GSE60102), and 4 hours (GSE27270) [173] and an RNA-seq study where THP-1 cells were treated with $1,25(\mathrm{OH})_{2} \mathrm{D} 3$ for $2.5,4$ and 24 hours ((GSE36323) [216]). Electron transport chain, oxidative phosphorylation and TCA cycle are only significantly altered $(\mathrm{Z}>1.96)$ in THP-1 cells treated with $1,25(\mathrm{OH})_{2} \mathrm{D} 3$ for 24 hours. Furthermore, cell cycle pathways related are only significantly altered $(\mathrm{Z}>1.96)$ in THP-1 cells of $1,25(\mathrm{OH})_{2} \mathrm{D} 324$ hours treatment cells from Nurminem and Vermay datasets. 


\subsection{Discussion}

Bioinformatics, or applying informatics to study biology, seeks to provide an unbiased and statistically robust insight into the molecular mechanisms that impact human health and disease. Large-scale projects such as the Human Genome Project [207] and ENCODE [52] have provided access to expansive repositories of data that have greatly helped to shed light on genomic function. However, utilization of these data repositories to answer specific biological questions remains limited. This is particularly true for studies of vitamin $\mathrm{D}$, despite detailed genome-wide analysis of VDR-chromatin interactions [191]. In most cases, analysis of the genomic responses to $1,25(\mathrm{OH})_{2} \mathrm{D} 3$-VDR complexes has been cell-specific, incorporating both classical calciotropic [160] and extra-skeletal [191] effects of vitamin D. Recently, a more integrated approach to the bioinformatic interrogation of $1,25(\mathrm{OH})_{2} \mathrm{D} 3$ VDR datasets has been described, utilizing VDR-chromatin immunoprecipitation-sequencing (ChIP-Seq) datasets with National Human Genome Research Institute (NHGRI) GenomeWide Association Study (GWAS) Single-Nucleotide Polymorphism (SNP) datasets [220]. This strategy of combining publicly available datasets presents a distinct set of challenges, notably in establishing statistical rigor and appropriate analytical workflows, but nevertheless, provides a novel perspective on the role of vitamin D and the VDR in human health. Notably, integration of ChIP-seq-GWAS datasets emphasized an important role for VDR in regulating of target genes associated with immunomodulation [220]. The current study proposes an alternative bioinformatic approach to further interrogate the immunomodulatory function of $1,25(\mathrm{OH})_{2} \mathrm{D} 3-\mathrm{VDR}$. Utilizing pathway analysis workflows to assess multiple RNA expression datasets for monocyte-derived cell models allowed to the identification of common cellular pathways that are regulated by $1,25(\mathrm{OH})_{2} \mathrm{D} 3$, revealing an important new immunometabolic function for vitamin D. The analysis pipeline described here offers an alternative statistical approach to conventional software packages. The most notable advantage of the workflow presented here is that it is open source and user-friendly for the bioscience community and enables reproducible and straightforward methodology that can process data from different gene expression analysis platforms.

Transcriptomic analysis of gene regulatory responses has played a pivotal role in defining the innate immune functions of vitamin D [47]. Notable studies have utilized specific monocyte [145] and dendritic cell [72] models to identify previously unrecognized molecular targets for active $1,25(\mathrm{OH})_{2} \mathrm{D} 3$, which have, in turn, revealed antibacterial $[4,14,46]$ and metabolic regulatory [244] functions for vitamin D in these cells. Collation of data from these different studies provides an alternative strategy for analysis of the immunomodulatory function of vitamin D, by incorporating groups of regulated genes into cellular pathway analyses. 
96 Pathway analysis of transcriptomic data shows immunometabolic effects of vitamin D

Using this strategy, it was possible to identify common cellular processes that are regulated by $1,25(\mathrm{OH})_{2} \mathrm{D} 3$, as well as those that are more cell lineage specific. The major advantage of this workflow is that it contextualizes the experimental data at the biological process level using interactive pathways. Based on criteria, the pathway statistics approach of PathVisio defines the genes that are significantly altered in the dataset and highlights the pathways of the WikiPathways repository that are altered after treatment with $1,25(\mathrm{OH})_{2} \mathrm{D} 3$. In our pathway analysis, we used the manually curated and up-to-date human pathway collection of WikiPathways containing a broad spectrum of biological processes, including well-described metabolic processes as well as signaling and gene regulatory processes. Pathway analysis not only highlights the altered biological processes based on changes in gene expression, but it enables the investigation of the relationship between genes and different datasets in great detail. This makes pathway analysis a suitable approach for an integrative and in-depth analysis of large-scale transcriptomic data.

The most striking observation from the current analysis is that in all three cell models, $1,25(\mathrm{OH})_{2} \mathrm{D} 3$ is strongly associated with changes in cellular metabolism, oxidative phosphorylation and energy generation. Oxidative phosphorylation has been shown to play an important role in promoting a tolerogenic phenotype in immune cells [246, 161]. Previous studies have reported effects of $1,25(\mathrm{OH})_{2} \mathrm{D} 3$ on mitochondrial functionality and physiology, and pathways related to glucose metabolism in monocyte-derived DCs [72]. In a similar fashion, serum $25(\mathrm{OH}) \mathrm{D}$ status has been linked to markers of bioenergetic pathways in human peripheral blood mononuclear cells [32], and active $1,25(\mathrm{OH})_{2} \mathrm{D} 3$ has been shown to increase production of ATP and ROS, as well as altering mitochondrial functionality and morphology by increasing its membrane potential and total mass of mitochondria in differentiating monocytes [72]. It was therefore notable in the current study that the most significantly altered pathways common to all three innate immune cell types were those associated with mitochondrial function: TCA cycle, electron transport chain and oxidative phosphorylation. Collectively, these observations underline an important role for $1,25(\mathrm{OH})_{2} \mathrm{D} 3$ as a positive regulator of mitochondrial metabolism and bioenergetic pathways in immune cells. Interestingly, this effect of $1,25(\mathrm{OH})_{2} \mathrm{D} 3$ appears to be consistent not only across the three myeloid cell models studied in detail in figure 4.2, but is also observed within different DC subtypes. In the current study, we chose an immature DC (iDC) model which focused purely on the effect of $1,25(\mathrm{OH})_{2} \mathrm{D} 3$, although this was for a longer time period (5 days) than the other models. Nevertheless, further analysis showed that the pathway effects of $1,25(\mathrm{OH})_{2} \mathrm{D} 3$ on iDC over 5 days were similar to those observed in immune-activated mature DC (mDC) treated with $1,25(\mathrm{OH})_{2} \mathrm{D} 3$ for $12 \mathrm{~h}$ (Supplementary Fig. 2). 
Pathways associated with glycolysis and gluconeogenesis, such as the TCA cycle, were also significantly regulated by $1,25(\mathrm{OH})_{2} \mathrm{D} 3$ in all three cell types studied. Catabolism of glucose leads to the formation of pyruvate, a biomolecule that is then metabolized in the TCA cycle. At present, relatively little is known about the impact of vitamin D on the TCA cycle, other than clinical studies showing association between vitamin D deficiency and dysregulation of glucose metabolism like diabetes [214]. The present study demonstrates that several genes involved in the glucose metabolism are significantly regulated by vitamin D figure 4.8. Comparison of the THP-1, DC and monocyte datasets showed that genes for enzymes such as hexokinase, phosphofructokinase and fructose-bisphosphatase are commonly upregulated after $1,25(\mathrm{OH})_{2} \mathrm{D} 3$ treatment. In contrast to oxidative phosphorylation and electron transport, the glycolysis/gluconeogenesis effects of $1,25(\mathrm{OH})_{2} \mathrm{D} 3$ were less consistent with some genes showing opposite patterns of regulation in different cell types. It is nevertheless clear that the regulation of glucose metabolism is a key facet of $1,25(\mathrm{OH})_{2} \mathrm{D} 3$ regulation of myeloid cells.

Besides common targets for vitamin D in innate immune cells, analysis of multiple repositories also highlighted pathways that were cell model specific. In THP-1 cells only, $1,25(\mathrm{OH})_{2} \mathrm{D} 3$ potently regulated genes involved in progression of cell cycle and DNA replication, consistent with the neoplastic origin of these cells. The pathways with high Z-score include retinoblastoma in cancer, G1 to $\mathrm{S}$ cell cycle control, DNA replication and cell cycle, are similar to those reported previously for prostate cancer cells treated with $1,25(\mathrm{OH})_{2} \mathrm{D} 3$ [132]. This suggests that, in addition to its common metabolic innate immune targets, $1,25(\mathrm{OH})_{2} \mathrm{D} 3$ also has common antiproliferative targets in neoplastic cell types, including suppression of key proteins that participate in progression of the cell cycle and regulation of DNA replication [129]. Interestingly, pathway analysis of $1,25(\mathrm{OH})_{2} \mathrm{D} 3$ in prostate cancer cells [132] did not reveal any significant regulation of the metabolic pathways as identified for monocytes and DCs in the current study. Thus, in the same way that suppression of cell cycle/DNA replication genes appears to be a cancer cell-specific effect of $1,25(\mathrm{OH})_{2} \mathrm{D} 3$, genes associated with oxidative phosphorylation and energy metabolism appear to be innate immune cell specific. Previous studies using THP-1 cells have also included analysis of the temporal variations in response to treatment with $1,25(\mathrm{OH})_{2} \mathrm{D} 3$ [216]. The datasets in this particular study did not conform to requirements for the comparative pathway analysis used in our current study. However, we were able to carry out a preliminary pathway analysis of these data (figure 4.15). Here, it was notable that metabolic and cell cycle effects of $1,25(\mathrm{OH})_{2} \mathrm{D} 3$ were only observed after $24 \mathrm{~h}$ of treatment, suggesting that pathway regulation by vitamin $\mathrm{D}$ is highly time dependent. 
98 Pathway analysis of transcriptomic data shows immunometabolic effects of vitamin D

The third major group of pathways shown to be regulated by $1,25(\mathrm{OH})_{2} \mathrm{D} 3$ in THP-1, monocytes and DCs were those associated with programmed cell death (apoptosis). Previous studies have reported pro-apoptotic effects of $1,25(\mathrm{OH})_{2} \mathrm{D} 3$ in primary cultures of monocytes, with this effect being mediated via interference with CD40 responses [9]. Conversely, in the $\mathrm{HL}-60$ leukemic cell line, $1,25(\mathrm{OH})_{2} \mathrm{D} 3$ was reported to promote resistance to apoptosis [168]. Furthermore, studies from our group have shown that antisense knockdown of VDR in another leukemic cell line, U937, promoted cell apoptosis [100]. Data from the current study further support a role for $1,25(\mathrm{OH})_{2} \mathrm{D} 3$ in regulating apoptosis of innate immune cells: common genes in the three datasets compared are commonly upregulated (TNFTSF10D) and downregulated (caspases family proteins and APAF1).

\subsection{Conclusions}

Understanding the entire set of results of high-throughput gene expression studies has often been challenging, caused by the complicated process of data analysis, and the limited biological interpretation that could be extracted from the large datasets generated through bench experiments. As a consequence, genome-wide analyses have tended to focus on changes in expression for specific genes [145, 47] or small groups of genes associated with a specific pathway [72]. The aim of the current study was to provide, for the first time, an unbiased analysis of the function of vitamin D in immune cells at a broader pathway level. Our resulting data demonstrate the added value of implementing a workflow with different bioinformatic tools that allows us to analyze gene expression data in a rapid, automated and reproducible fashion, to highlight pathways that are significantly altered by $1,25(\mathrm{OH})_{2} \mathrm{D} 3$. It is possible to visualize and further interrogate these biological diagrams to provide a detailed description of the molecular effects of $1,25(\mathrm{OH})_{2} \mathrm{D} 3$ in different types of immune cells. Finally, comparison of different datasets has allowed us to identify pathways, notably those associated with cell metabolism, that are common to multiple different types of innate immune cells. This approach provides new insights into the immunomodulatory actions of vitamin $\mathrm{D}$, but also has important implications for the many other physiological responses linked to vitamin $\mathrm{D}$ in recent studies. 


\section{Chapter 5}

\section{Tolerogenic effects of vitamin D on DCs involve induction of fatty acid synthesis}

Submitted to Journal of Endocrinology in 2020

Amadeo Muñoz García ${ }^{1,2}$, Danyang $\mathrm{Li}^{1,2}$, Emma Bishop $^{1,3}$, Louisa Jeffery ${ }^{4}$, Antje Garten ${ }^{5}$, Alpesh Thakker ${ }^{1}$, Michelangelo Certo ${ }^{6}$, Claudio Mauro ${ }^{1,6}$, Daniel A. Tennant ${ }^{1}$, Sarah Dimeloe $^{1,3}$, Chris T. Evelo ${ }^{2,7}$, Susan L. Coort ${ }^{2}$, Martin Hewison ${ }^{1}$

\footnotetext{
${ }^{1}$ Institute of Metabolism and Systems Research, The University of Birmingham, Birmingham, B15 2TT, United Kingdom

${ }^{2}$ Department of Bioinformatics - BiGCaT, NUTRIM School of Nutrition and Translational Research in Metabolism, P.O. Box 616, Maastricht University, Maastricht, the Netherlands ${ }^{3}$ Institute of Immunity and Immunotherapy, University of Birmingham, Birmingham, B15 2TT, United Kingdom

${ }^{4}$ Institute of Translational Medicine, University of Birmingham, Birmingham, B15 2TT, United Kingdom

${ }^{5}$ Universität Leipzig, Medizinische Fakultät, 04103 Leipzig, Germany

${ }^{6}$ Institute of Inflammation and Ageing, University of Birmingham, Birmingham, B15 2TT, United Kingdom

${ }^{7}$ Maastricht Centre for System Biology (MaCSBio), Maastricht University, Maastricht, Netherlands
} 


\subsection{Abstract}

The active form of vitamin $\mathrm{D}, 1,25$-dihydroxyvitamin $\mathrm{D}\left(1,25(\mathrm{OH})_{2} \mathrm{D} 3\right)$ is a potent regulator of innate immune function, promoting anti-inflammatory, tolerogenic $\mathrm{T}$ cell responses by modulating antigen presentation by dendritic cells (DC). Transcriptomic analyses indicate that DC responses to $1,25(\mathrm{OH})_{2} \mathrm{D} 3$ involve changes in glycolysis, oxidative phosphorylation, electron transport and the TCA cycle. To determine the functional impact of metabolic remodelling on innate immune responses to $1,25(\mathrm{OH})_{2} \mathrm{D} 3$, human monocyte-derived DC were differentiated to immature (+vehicle, iDC), mature (+LPS, mDC), and immature tolerogenic DC $\left(+1,25(\mathrm{OH})_{2} \mathrm{D} 3\right.$, itolDC) and characterised for metabolic function. In contrast to $\mathrm{mDC}$ which showed suppression of maximal respiration capacity, itolDC showed increased basal and maximal respiration capacity relative to iDC. Tracer metabolite analyses using ${ }^{13} \mathrm{C}_{6}$-labeled glucose showed increased generation of lactate and TCA cycle metabolites. When glutamine was used as a TCA cycle substrate, the effects of $1,25(\mathrm{OH})_{2} \mathrm{D} 3$ were less significant, indicating that $1,25(\mathrm{OH})_{2} \mathrm{D} 3$ primarily stimulates glycolysis and TCA cycle metabolism. Analysis of non-polar lipophilic metabolites of ${ }^{13} \mathrm{C}_{6}$-glucose revealed significant incorporation of label in palmitate and palmitoleate, suggesting the $1,25(\mathrm{OH})_{2} \mathrm{D} 3$ promotes metabolic remodelling and fatty acid synthesis in itolDC. Inhibition of fatty acid synthesis in itolDC altered itolDC morphology and suppressed expression of CD14 and IL-10 by these cells. These data indicate that the ability of $1,25(\mathrm{OH})_{2} \mathrm{D} 3$ to induce tolerogenic DC is dependent on metabolic remodelling leading to synthesis of fatty acids.

\subsection{Introduction}

The active form of vitamin $\mathrm{D}, 1,25$-dihydroxyvitamin $\mathrm{D}\left(1,25(\mathrm{OH})_{2} \mathrm{D} 3\right)$, is a potent immunomodulator, promoting innate antibacterial activity [98] [99], whilst inhibiting inflammatory acquired immune responses [99] [3]. Similar to its classical calciotropic actions, the immunomodulatory activities of $1,25(\mathrm{OH})_{2} \mathrm{D} 3$ are mediated by binding to the nuclear vitamin D receptor (VDR) [96], and concomitant regulation of transcription [47]. Whilst VDR expression is ubiquitous, the level of VDR is known to vary significantly in cells from the immune system. Resting T cells exhibit very low levels of VDR, with expression increasing dramatically following exposure to immunogens and T cell activation [20]. In this way $1,25(\mathrm{OH})_{2} \mathrm{D} 3$ is able to regulate activated $\mathrm{T}$ cells directly by suppressing $\mathrm{T}$ helper 1 (Th1) and Th17 function and promoting differentiation into regulatory T cells (Treg) [110]. However, $1,25(\mathrm{OH})_{2} \mathrm{D} 3$ can also influence adaptive immunity $\mathrm{T}$ cell function via indirect effects on antigen presenting cells. Macrophages [128] and dendritic cells (DC) [101] express 
VDR, together with the enzyme that synthesises $1,25(\mathrm{OH})_{2} \mathrm{D} 3,1 \alpha$-hydroxylase (CYP27B1). In macrophages, exogenously added or endogenously generated $1,25(\mathrm{OH})_{2} \mathrm{D} 3$ acts via VDR to stimulate antibacterial responses, including induction of antimicrobial peptides [145], enhanced autophagy [263], and regulation of intracellular iron [15]. In DC, 1,25(OH) 2 D3 suppresses IL-12 production [101] and CD80/CD86 expression [101], but increases IL-10 production [240]. The overall effect of $1,25(\mathrm{OH})_{2} \mathrm{D} 3$ is to maintain an immature status in DC [101] [190], thereby promoting a tolerogenic DC phenotype (tolDC) [171] [186] which can elicit development of Treg [5].

The mechanisms by which $1,25(\mathrm{OH})_{2} \mathrm{D} 3$ modulates DC function have still to be fully elucidated, and are likely to be dependent on specific DC phenotype. In previous studies we have shown that differentiation of monocytes to immature DC (iDC) is associated with decreased VDR and elevated CYP27B1 expression [101]. In this in vitro model, further differentiation of iDC to mature $\mathrm{DC}(\mathrm{mDC})$ following CD40-ligation had no further effect on VDR expression [101]. Similar observations have also been reported for iDC matured to $\mathrm{mDC}$ with lipopolysaccharide (LPS) [79]. Most published studies of vitamin D responses in DC have focused on LPS-induced $\mathrm{mDC}$ to define a role for $1,25(\mathrm{OH})_{2} \mathrm{D} 3$ as a regulator $\mathrm{mDC}$ function [131] [30]. In studies using murine bone marrow-derived DC, 1,25(OH) ${ }_{2} \mathrm{D} 3$ was shown to regulate a greater number of genes in LPS-induced $\mathrm{mDC}$ relative to vehicle-treated iDC [213]. Whilst some genes were commonly regulated by $1,25(\mathrm{OH})_{2} \mathrm{D} 3$ in iDC and $\mathrm{mDC}$, it was clear that many $1,25(\mathrm{OH})_{2} \mathrm{D} 3$-regulated genes were specific to $\mathrm{mDC}$ or iDC [213]. Thus, the maturation context of DC responses to $1,25(\mathrm{OH})_{2} \mathrm{D} 3$ is likely to be critical in defining the impact of vitamin $\mathrm{D}$ on innate immune DC function.

Recent reports have shown that the effects of $1,25(\mathrm{OH})_{2} \mathrm{D} 3$ on $\mathrm{mDC}$ are crucially dependent on metabolic remodelling. In monocyte-derived, LPS-induced $\mathrm{mDC}$, pathway analyses showed that the dominant transcriptomic effects of $1,25(\mathrm{OH})_{2} \mathrm{D} 3$ involved changes in glucose metabolism, oxidative phosphorylation and the TCA cycle [72]. Specifically, short-term treatment of LPS-induced $\mathrm{mDC}$ with $1,25(\mathrm{OH})_{2} \mathrm{D} 3$ induced a mature tolDC (mtolDC) phenotype that was dependent on glucose availability and glycolysis [72]. In studies using multiple gene expression repositories we have shown that the ability of $1,25(\mathrm{OH})_{2} \mathrm{D} 3$ to promote metabolic remodelling via glycolysis, oxidative phosphorylation and the TCA cycle is common to many myeloid cell types including monocytes, iDC and $\mathrm{mDC}$ [81]. In the current study we have further defined the role of DC metabolic remodelling in mediating immunomodulatory effects of vitamin $\mathrm{D}$ by demonstrating increased fatty acid synthesis in $1,25(\mathrm{OH})_{2} \mathrm{D} 3$-induced immature tolDC (itolDC) in the absence of immunogenic stimulus. Inhibition of DC fatty 
acid synthase suppressed the ability of $1,25(\mathrm{OH})_{2} \mathrm{D} 3$ to promote IL-10-secreting itolDC, indicating that regulation of lipid metabolism is central to the immunomodulatory actions of vitamin D.

\subsection{Materials \& Methods}

\subsubsection{Isolation of primary human peripheral blood monocytes}

Healthy human peripheral blood mononuclear cells (PBMC) were obtained from fully anonymised blood cones obtained from the National Blood Service, Birmingham, UK, in accordance with ethical agreement ERN_14-0446. PBMC were isolated from whole blood leukocyte cones from healthy donors using LymphoPrep Separation Media (Stem Cell Technologies, Cambridge, UK) as per manufacturer's instructions, and resuspended in ice-cold MACS (magnetic-activated cell sorting) buffer to achieve approximately 50million PBMCs per mL. CD14+ monocytes were isolated from PBMC using the EasySep Monocyte Isolation Kit (Stem Cell Technologies) as per manufacturer's instructions. The yield of CD14+ monocytes achieved from 500 million PBMC was approximately 50 million. The resulting monocytes were transferred to a new tube, and PBS added up to $15 \mathrm{~mL}$. Following centrifugation at 1500rpm for 5 minutes, pelleted CD14+ monocytes were re-suspended in Roswell Park Memorial Institute (RPMI) 1640 Medium (Thermofisher, Loughborough, UK) supplemented with 5\% L-glutamine (Sigma Aldrich, Gillingham, UK) and 10\% foetal bovine serum (FBS; Biosera, Heathfield, UK) to achieve 2 million cells per mL.

\subsubsection{In vitro generation of monocyte-derived DC}

CD14+ monocytes were differentiated into DC in vitro according to the schematic shown in figure 5.1 A. Monocytes were cultured for 5 days with granulocyte macrophage-stimulating colony factor (GM-CSF; $800 \mathrm{U} / \mathrm{mL}$, Berlex Laboratories, Seattle, WA) and Interleukin-4 (IL-4; $400 \mathrm{U} / \mathrm{mL}$ ), in the presence or absence of $10 \mathrm{nM} 1,25(\mathrm{OH})_{2} \mathrm{D} 3$ (Enzo Life Sciences, Exeter, UK; diluted in RPMI 1640 medium from $50 \mu \mathrm{g} / \mathrm{mL}$ stock), at $37^{\circ} \mathrm{C}$ and $5 \% \mathrm{CO}_{2}$. Fresh medium supplemented with GM-CSF and IL-4 was added on day 2 and day 5 of culture, with the resulting day 6 cells being iDC. The addition of $1 \mu \mathrm{g} / \mathrm{mL}$ LPS (from E. coli, Sigma Aldrich) for 24 hrs on day 6 generated mature DC (mDC). Addition of 1,25(OH) 2 D3 $(10 \mathrm{nM})$ for all 6 days of culture in the absence of LPS was used to generate itolDC and the addition of LPS for the last $24 \mathrm{hrs}$ of these cultures generated mature tolerogenic DC (mtolDC). In some cultures mitochondrial fatty acid synthase (FAS) was specifically inhibited using 25 $\mu \mathrm{M}$ of 4-Methylene-2-octyl-5-oxotetrahydrofuran-3-carboxylic acid (C75, Sigma Aldrich) 
from day 0 . Refresh of media, with the corresponding factors and treatments, was done in day 2 .

\subsubsection{Flow cytometry analysis of DC}

Approximately 50,000 cells were transferred directly from culture plates into flow cytometry tubes, washed once with PBS and centrifuged at 1500rpm for 5 minutes. After removing the supernatant, each sample was re-suspended by gentle vortex in $100 \mu \mathrm{L}$ LIVE/DEAD Fixable Near-IR Dead Cell Stain ( $1 \mu \mathrm{L}$ stock in 1mL PBS; Life Technologies) to stain for dead cells. Samples were incubated in the dark on ice for 20 mins, and then washed with PBS and the supernatant discarded. Fluorescent cell surface staining antibodies were added to samples to a total of $100 \mu \mathrm{L}$ per sample in PBS. Following incubation on ice for 30 mins, samples were washed again and the resulting pellet re-suspended in $200 \mu \mathrm{L}$ PBS for immediate flow cytometry analysis, or fixed for subsequent analysis at a later date. All washing and staining stages were carried out in the absence of light. For all studies cells were acquired on a Dako Cyan flow cytometer (Dako Cytomation), median fluorescent intensity (MFI) was measured for each cell surface marker and data were analysed using FlowJo software (Tree Star version 8.8.6). Data were also shown as representative flow cytometry plots. All antibodies were purchased from ebioscience/Thermofisher or BD Biosciences and expression quantified relative to the appropriate isotype control. A full list of antibodies, cytokines, volumes used and product numbers is shown in table 5.1. Rat IgG antibodies conjugated with matching fluorophores were used for the isotype control stain, and compensation colours were also added. 


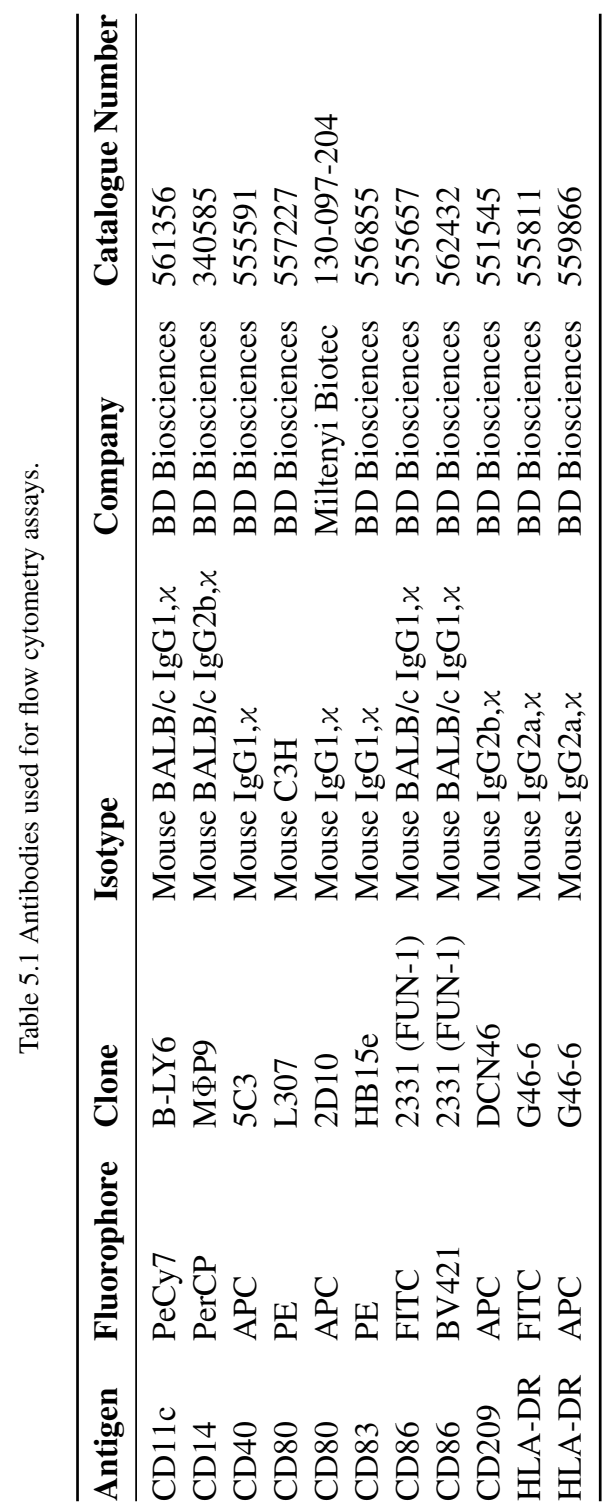




\subsubsection{Microscope fluorescence imaging}

For fluorescence microscopy imaging DCs were washed with PBS and stained with 100 nM MitoTracker Green FM (Invitrogen) diluted in RPMI 1640 medium without FBS at $37^{\circ} \mathrm{C}$ for $25 \mathrm{~min}$. Cells were washed and covered with mounting medium containing DAPI (VECTASHIELD) for nuclear staining. Images were taken using the Zeiss LSM780 confocal microscope. Two channels were selected: excitation at $490 \mathrm{~nm}$ - emission 416 for mitochondria stain based on MitoTracker Green FM and excitation at $360 \mathrm{~nm}$ and emission at $460 \mathrm{~nm}$ for nuclei stained with DAPI.

\subsubsection{Quantitative Real-time PCR}

Total RNA was extracted by phenol/chloroform method after cell lysis in TRIzol (Life Technologies/Invitrogen). 0.3-0.5 $\mu$ g RNA was reverse transcribed with random hexamers using TaqMan reverse transcription reagents (Thermofisher/Applied Biosystems). Quantitative real-time PCR for 18S rRNA, VDR, CYP24A1 and CYP27B1 was then performed on an Applied Biosystems 7900 machine using assays on demand from Applied Biosystems: 18S rRNA, (4319413E); VDR (Hs00118624 CE); CYP24A1 (Hs00167999_m1); CYP27B1 (Hs01096154_m1). Amplification of cDNAs involved incubation at $50^{\circ} \mathrm{C}$ for 2 mins and $95^{\circ} \mathrm{C}$ for $10 \mathrm{mins}$ followed by 40 cycles of $95^{\circ} \mathrm{C}$ for 15 secs and $60^{\circ} \mathrm{C}$ for $1 \mathrm{~min}$. mRNA expression was statistically analysed as raw delta $\mathrm{Ct}$ values, and also reported as fold-change in mRNA expression calculated as described previously [4].

\subsubsection{Measurement of oxygen consumption}

Measurement of mitochondrial oxygen consumption were performed based on previously reported studies [188] [83]. Cultured DC were used in respiration assays (3-5 x10 5 per assay) to study the effects of $1,25(\mathrm{OH})_{2} \mathrm{D} 3$ on the electron transport chain (ETC) and oxidative phosphorylation. Samples were analysed using an Oroboros O2k-FluoRespirometer (Oroboros Inc., Innsbruck, Austria) following the sequential addition of different compounds as follows: oligomycin at $2.5 \mu \mathrm{M}$ (inhibitor of ATP synthase - measure ATP-coupled respiration); FCCP at $1 \mu \mathrm{M}$, until maximal respiration; rotenone at $0.5 \mu \mathrm{M}$ (inhibitor of complex I - measures complex II linked respiration); antimycin A at $2.5 \mu \mathrm{M}$ (inhibitor of complex III - stops respiration and measures residual oxygen consumption). Raw oxygen consumption rate changes were registered and processed to estimate different bioenergetic related parameters such as basal respiration, ATP-linked respiration and maximal capacity of ETC. 


\subsubsection{Mass spectrometry analysis of ${ }^{13} C_{6}$-glucose and ${ }^{13} C_{5}$-glutamine metabolism}

To assess effects of $1,25(\mathrm{OH})_{2} \mathrm{D} 3$ on glycolysis and the TCA cycle metabolic tracer analyses were carried out using uniformly-labeled ${ }^{13} \mathrm{C}_{6}$-glucose or ${ }^{13} \mathrm{C}_{5}$-glutamine as substrates as described previously [174]. Briefly, in the last 24 hours of cell culture, conventional medium was replaced by DMEM supplemented with $10 \% \mathrm{FBS}$, $\left[\mathrm{U}_{-}{ }^{13} \mathrm{C}\right]$ glutamine $(2 \mathrm{mM})$ or glucose $(10 \mathrm{mM})$. Following culture, cells were harvested and washed with $\mathrm{NaCl}(0.1 \%)$. Metabolite extraction was performed by a gradient of 2.5 parts methanol:2.5 parts of chloroform:0.625 parts of $\mathrm{H} 2 \mathrm{O}$ containing D-6-acid glutaric as internal reference. The extracted polar fraction was analysed to study metabolism in glycolysis and TCA cycle. For analysis of fatty acid synthesis, the extracted non-polar fraction was utilised. In the case of the latter, the replacement of RPMI media with DMEM media was carried out in day 3 of cell culture. In this case, a gradient of 50\% chloroform:50\% methanol containing C17:0, heptadecylic acid (Sigma Aldrich) was used as an internal reference. The non-polar fraction was extracted and dried with nitrogen gas. Metabolites that differ in mass due to carbon composition are known as isotopologues and were represented as $\mathrm{M}+\mathrm{n}$; where $\mathrm{n}$ is the number of ${ }^{13} \mathrm{C}$ atoms incorporated. Using mass spectrometry, metabolites and their isotopologues were quantified and their relative abundance was calculated. Metabolite abundance was normalized by the internal standard and total ion count.

\subsubsection{Statistical analysis}

GraphPad Prism 7.0a software (GraphPad) was used for graphical summary and statistical analysis. Oxygraph raw data were analysed statistically using a paired sample student t-test to determine the effects of each treatment ( $\mathrm{p}$ value for significance $<0.05$ ). For metabolite tracer experiments, a paired sample multi t-test was applied for each isotopologue and statistical significance was determined using the Holm-Sidak method with alpha= 0.05 without assuming consistent standard deviation. FACS and ELISA data was statistically tested using one-way ANOVA with post-hoc Tukey's test. For each test a 95\% confidence level was used. 


\subsection{Results}

\subsection{1 $1,25(\mathrm{OH})_{2} \mathrm{D3}$ regulates $\mathrm{DC}$ phenotype in the presence and ab- sence of immunogenic stimulus}

Differentiation of human peripheral blood monocytes to DC using $1,25(\mathrm{OH})_{2} \mathrm{D} 3$ in the presence or absence of LPS was associated with specific changes in DC phenotype (figure 5.1). Relative to vehicle-treated iDC, LPS-induced mDC showed increased numbers of HLA-DR+ cells, and increased MFI for CD11c, CD80, CD83, CD86, CD209 and HLA-DR (figure 5.1 B and C). Relative to $\mathrm{mDC}$, mtolDC demonstrated decreased HLA-DR positivity, and suppressed CD80, CD83, CD86, CD209 and HLA-DR MFI but increased CD14 MFI (figure 5.1 B and C). Relative to iDC, $1,25(\mathrm{OH})_{2} \mathrm{D} 3$-induced itolDC showed decreased numbers of CD80+ and HLA-DR+ cells, whilst increasing CD14+ cells. The MFI for CD80, CD86 and HLA-DR was also decreased in itolDC relative to iDC, whilst CD14 MFI was increased (figure 5.1 B and C). Analysis of DC gene expression was carried out using raw delta $\mathrm{Ct}$ values for statistical analysis and fold-change values relative to iDC for visualisation are shown in figure 5.1 D. Expression of mRNA for VDR (2.3-fold) and CYP27B1 (75.5-fold) was significantly higher in $\mathrm{mDC}$ relative to iDC, and this was unaffected by co-treatment with 1,25(OH) $)_{2} \mathrm{D} 3$ (mtolDC, 3.4- and 55.7-fold increases respectively relative to iDC) (figure 5.1 D). By contrast, 6 day exposure to $1,25(\mathrm{OH})_{2} \mathrm{D} 3$ in itolDC had no effect on VDR or CYP27B1 mRNA expression relative to vehicle-treated iDC (figure 5.1 D). For the vitamin D-target gene CYP24A1, mRNA expression was profoundly suppressed in $\mathrm{mDC}$ relative to iDC (10-fold), but CYP24A1 mRNA was increased in itolDC (7.4-fold) and $\mathrm{mDC}+1,25(\mathrm{OH})_{2} \mathrm{D} 3$ (20.2-fold).

\subsubsection{1,25 $(\mathrm{OH})_{2} \mathrm{D3}$-induced itolDC are characterised by increased ox- idative phosphorylation}

Oxygen consumption rates (OCR) were measured in different populations of DC to assess the effects of $1,25(\mathrm{OH})_{2} \mathrm{D} 3$ and LPS on metabolic remodelling in the different DC phenotypes (figure 5.2). Relative to iDC, $1,25(\mathrm{OH})_{2} \mathrm{D} 3$-induced itolDC showed increased basal respiration and maximal respiration (figure 5.2 $\mathrm{A}$ and $\mathrm{C}$ ). However, the enhanced oxygen consumption induced by $1,25(\mathrm{OH})_{2} \mathrm{D} 3$ in itolDC was unaffected by co-treatment with LPS to induce mtolDC (figure 5.2 B and D). The effects of $1,25(\mathrm{OH})_{2} \mathrm{D} 3$ on OCR were associated with long-term treatment, as iDC or mDC treated with $1,25(\mathrm{OH})_{2} \mathrm{D} 3$ for $24 \mathrm{hrs}$ showed no significant changes in OCR (data not shown). 
A.

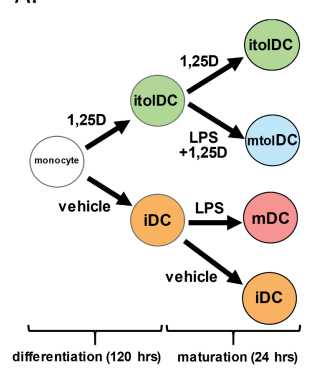

B.

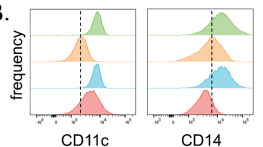

C.

D.
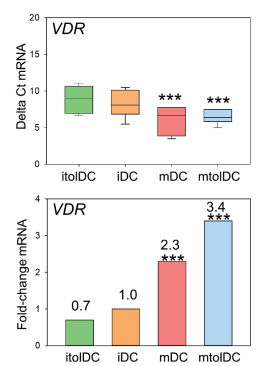

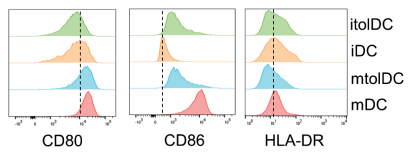

CD80

CD86
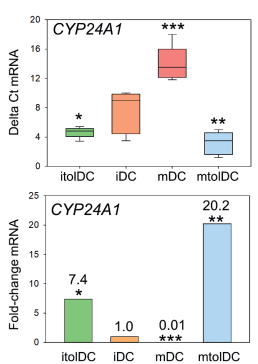
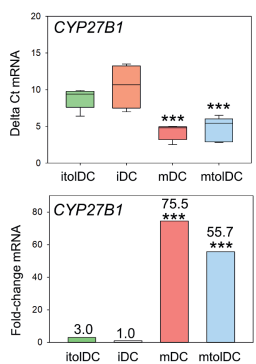

Fig. 5.1 Regulation of DC phenotype by $1,25(\mathrm{OH})_{2} \mathrm{D} 3$. Schematic representation of the model for cell culture of human monocyte-derived DC. Immature DC (iDC), mature DC (mDC), immature tolerogenic DC (itolDC), mature tolerogenic DC (mtolDC). B. Representative flow cytometry analyses for CD11c, CD14, CD80, CD86 and HLA-DR in iDC, $\mathrm{mDC}$, mtolDC and itolDC showing comparison between iDC vs itolDC, iDC vs $\mathrm{mDC}$ and $\mathrm{mDC}$ vs mtolDC. C. Median fluorescence intensity values for CD11c, CD14, CD40, CD80, CD83, CD86, CD209 and HLA-DR in iDC, mDC, mtolDC and itolDC. D. Expression of mRNA for VDR, CYP24A1 and CYP27B1 in iDC, mDC, mtolDC and itolDC. Data are shown as: raw delta Ct values with associated statistical analysis (upper panel); fold-change in mRNA expression (lower panel). Data show median and upper/lower quartiles for $\mathrm{n}=5$ replicate donor analyses. $*=$ statistically different from iDC values, $\mathrm{p}<0.05$, $* * \mathrm{p}<0.01$, *** $\mathrm{p}<0.001$. 
A.

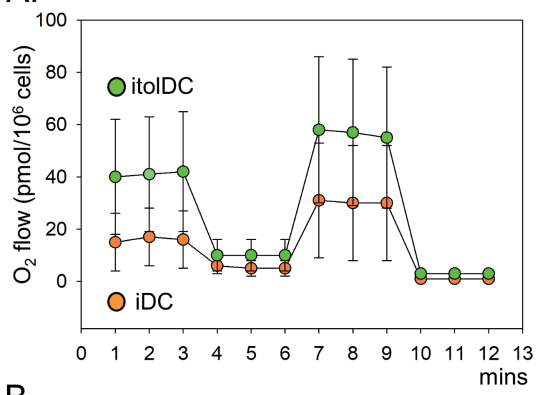

B.

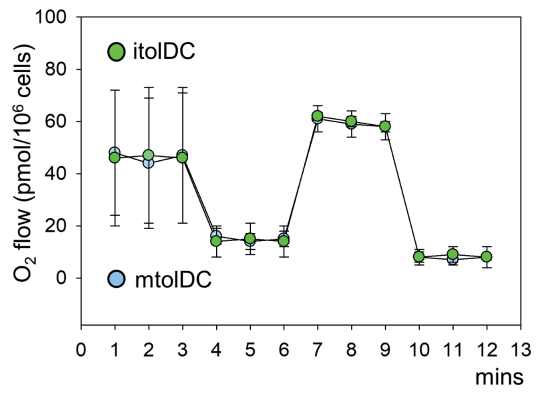

C.

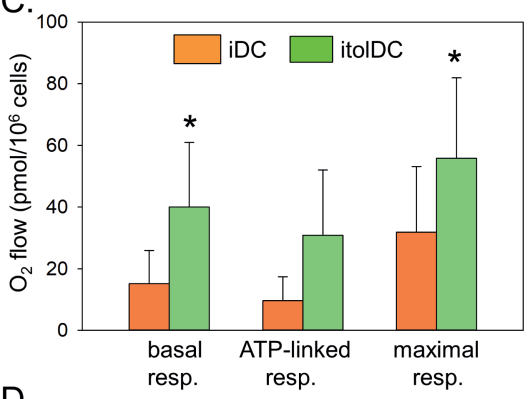

D.

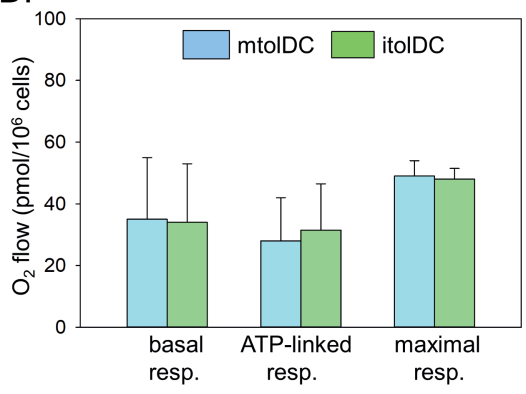

Fig. 5.2 1,25(OH $)_{2}$ D3-induced oxidative phosphorylation in itolDC. A\&B. Representative traces for: A. itolDC vs iDC; C. itolDC vs mtolDC showing changes in oxygen consumption rate (OCR) under conditions of: i) basal respiration; ii) oligomycin-induced inhibition of ATP production; iii) FCCP uncoupling of electron transport chain; iv) antimycin A/rotenone inhibition of electron transport. C\&D. Replicate basal respiration, ATP-linked respiration and maximal respiration capacity data for: C. iDC vs itolDC; D. itolDC vs mtolDC. Data are the mean $\pm \mathrm{SD}$ for $\mathrm{n}=4$ separate donor monocyte preparations. $*=$ statistically different from iDC values, $\mathrm{p}<0.05$. 
A.

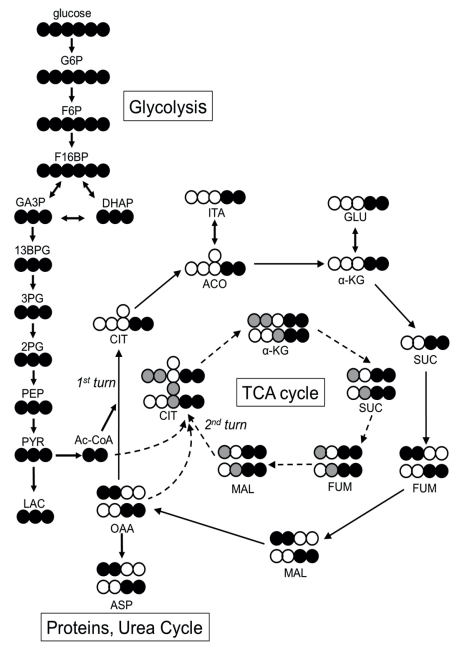

B.
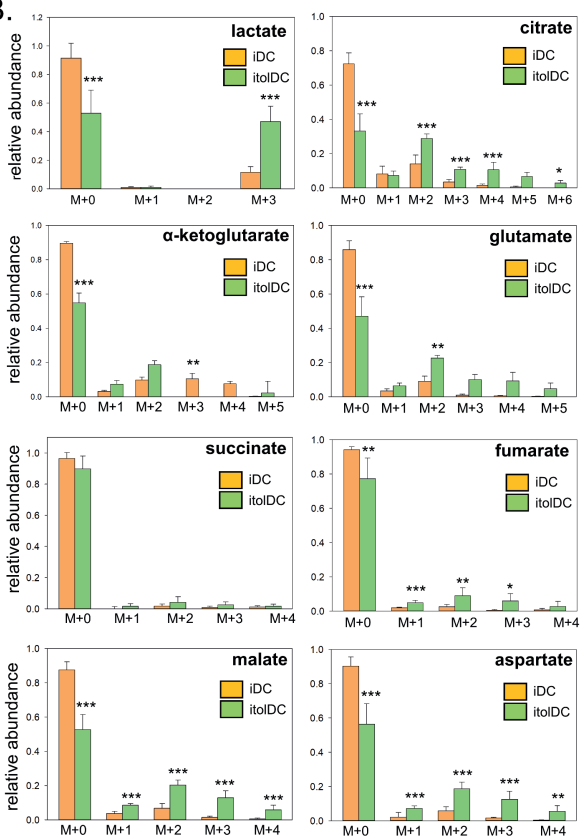

C.
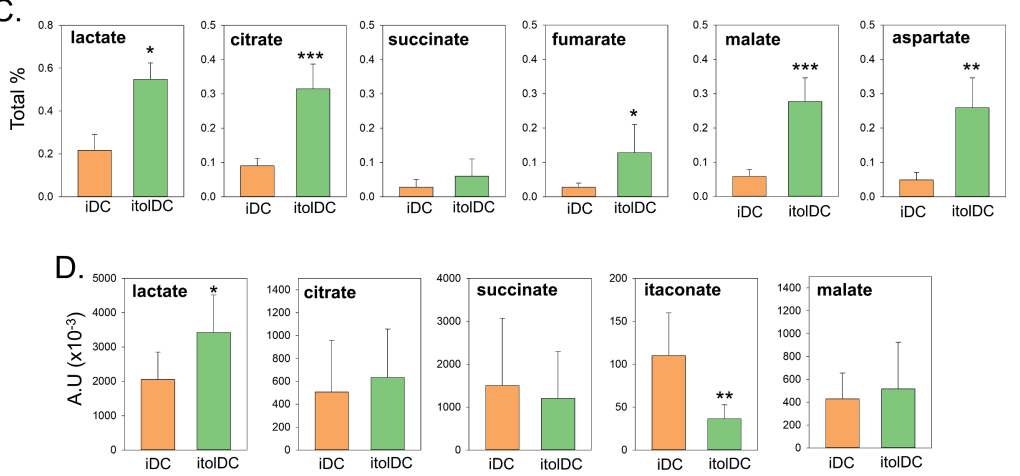

Fig. 5.3 Tracer metabolite analysis of glucose metabolism in iDC and itolDC. A. Schematic representation for incorporation of ${ }^{13} \mathrm{C}_{6}$-labeled glucose in polar TCA cycle metabolites through glycolysis and TCA cycle metabolism. B. Relative abundance of unlabelled $(\mathrm{M}+0)$ and ${ }^{13} \mathrm{C}$-labeled $(\mathrm{M}+1, \mathrm{M}+2, \mathrm{M}+3, \mathrm{M}+4, \mathrm{M}+5$, $\mathrm{M}+6)$ TCA cycle metabolites in iDC and itolDC. C. Total \% of TCA cycle metabolites in iDC vs itolDC produced due to glucose consumption. D. Total pool of TCA cycle metabolites in iDC vs itolDC (arbitrary units A.U.). Abbreviations: glucose 6-phosphate (G6P); fructose 6-phosphate (F6P); fructose 1,6-bisphosphate (F16BP); glyceraldehyde 3-phosphate (GA3P); dihydroacetone phosphate (DHAP); 1,3-bisphosphoglyceric acid (13BPG); 3-phosphoglyceric acid (3PG); 2-phosphoglyceric acid (3PG); phosphoenolpyruvic acid (PEP); pyruvate (PYR); lactate (LAC); acetyl-CoA (Ac-CoA); citrate (CIT); aconitate (ACO); itaconate (ITA); $\alpha$ ketoglutarate $(\alpha-K G)$; glutamate (GLU); succinate (SUC); fumarate (FUM); malate (MAL); oxalo-acetate (OXA); aspartate (ASP). Data are mean $\pm \mathrm{SD}$ for $\mathrm{n}=5$ donor monocyte preparations. $*=$ itolDC values statistically different from iDC, $\mathrm{p}<0.05, * * \mathrm{p}<0.01, * * * \mathrm{p}<0.001$. 


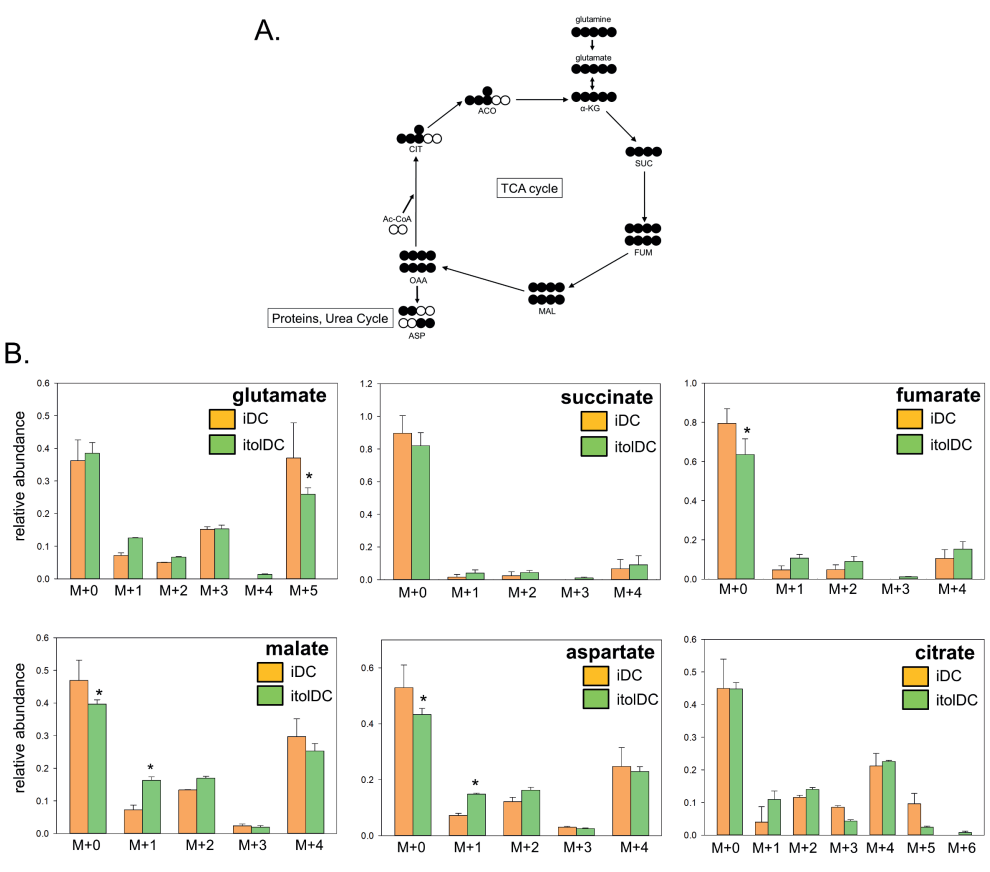

B.

Fig. 5.4 Tracer metabolite analysis of glutamine metabolism in iDC and itolDC. Schematic representation showing incorporation of ${ }^{13} \mathrm{C}_{5}$-labeled glutamine in polar TCA cycle metabolites as a consequence of TCA cycle metabolism. B. Relative abundance of unlabelled $(\mathrm{M}+0)$ and ${ }^{13} \mathrm{C}$-labeled $(\mathrm{M}+1, \mathrm{M}+2, \mathrm{M}+3, \mathrm{M}+4, \mathrm{M}+5$, $\mathrm{M}+6$ ) TCA cycle metabolites in iDC and itolDC. Abbreviations: acetyl-CoA (Ac-CoA); citrate (CIT); aconitate (ACO); itaconate (ITA); $\alpha$-ketoglutarate ( $\alpha$-KG); glutamate (GLU); succinate (SUC); fumarate (FUM); malate (MAL); oxalo-acetate (OXA); aspartate (ASP). Data are the mean $\pm \mathrm{SD}$ for $\mathrm{n}=5$ separate donor monocyte preparations. ${ }^{*}=$ itolDC values statistically different from iDC, $\mathrm{p}<0.05, * * \mathrm{p}<0.01, * * * \mathrm{p}<0.001$. 

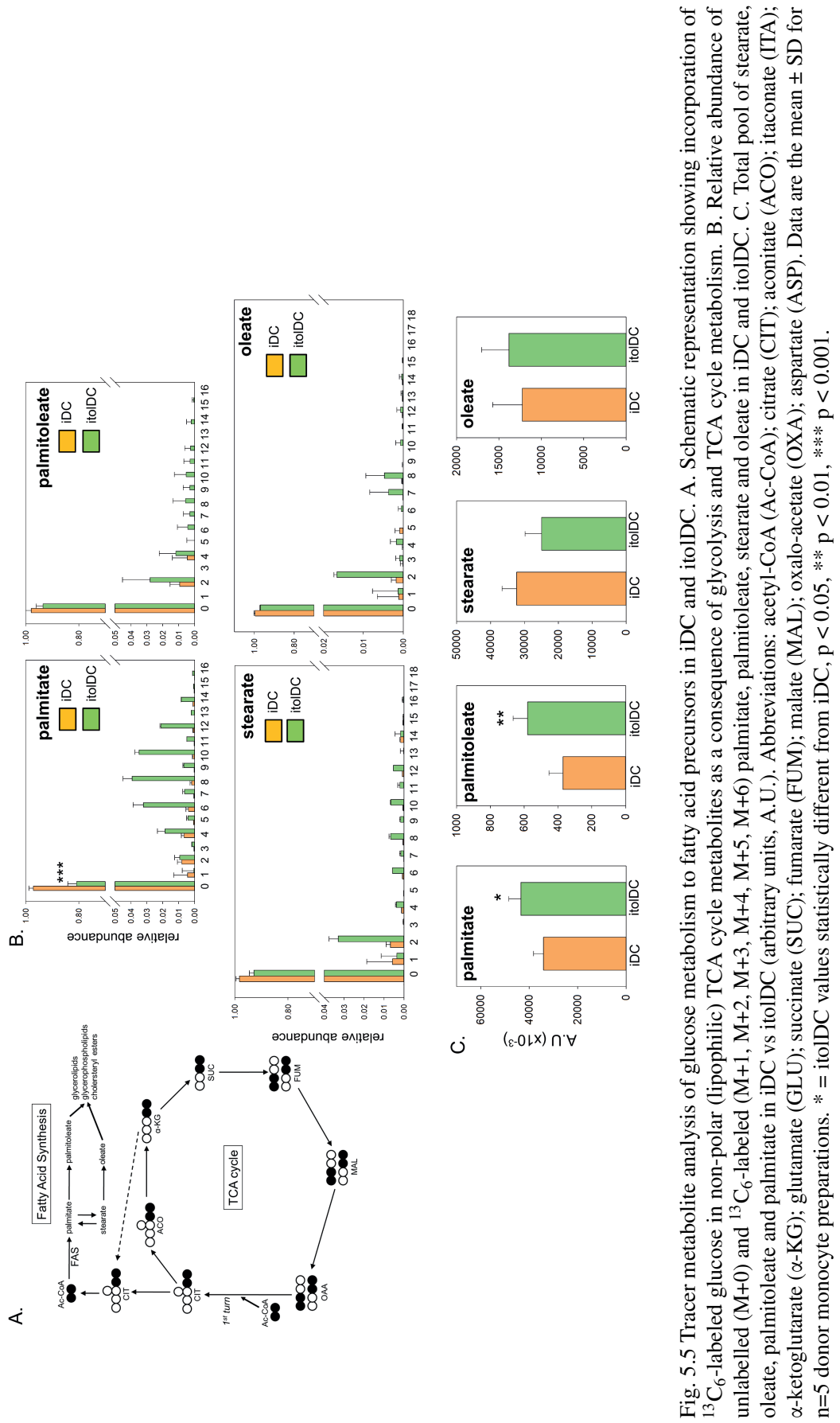


\subsubsection{1,25(OH $)_{2}$ D3-induced itolDC show increased TCA cycle metabolism}

Data in figures 5.1 and 5.2 indicate that DCs treated with $1,25(\mathrm{OH})_{2} \mathrm{D} 3$ alone show an enhanced tolerogenic phenotype (itolDC) which was associated with increased mitochondrial respiration. Further studies were therefore carried out to more precisely define the metabolic remodelling induced by $1,25(\mathrm{OH})_{2} \mathrm{D} 3$ in itolDC. DCs treated with fully labelled $\mathrm{U}^{13} \mathrm{C}_{6}$-glucose for the final 24 hours of culture were analysed by mass spectrometry to quantify incorporation of the isotope into the resulting polar (hydrophilic) tracer metabolites (figure 5.3). The pathway for incorporation of ${ }^{13} \mathrm{C}_{6}$-glucose into metabolites via glycolysis and the TCA cycle is shown in figure $5.3 \mathrm{~A}$. Following glycolysis, the carbons from ${ }^{13} \mathrm{C}_{2}$ acetyl-CoA are committed to the TCA cycle through citrate synthase and remain in every metabolite generated in the first round of the cycle, reflected in the relative abundance of $\mathrm{M}+$ 2 isotopomers (figure 5.3 B). Further incorporation of ${ }^{13} \mathrm{C}_{2}$-acetyl-CoA in a second round of the TCA cycle generates $\mathrm{M}+4$ and $\mathrm{M}+6$ isotopomers of citrate which were also increased in itolDC. $\mathrm{M}+3$ and $\mathrm{M}+5$ isotopomers reflecting consecutive rounds of TCA cycle and pyruvate carboxylase activity were also enhanced in itolDC (figure 5.3 B).

Consistent with the $1,25(\mathrm{OH})_{2} \mathrm{D} 3$-induced glycolysis and TCA cycle shown in figure $5.3 \mathrm{~B}$, itolDC also showed higher incorporation of glucose carbons into citrate $(66.91 \%)$, aspartate $(43.72 \%)$, malate $(47.37 \%)$, fumarate $(22.71 \%)$ and lactate $(47.09 \%)$ relative to vehicletreated iDC $(27.69 \%, 9.79 \%, 12.53 \%, 5.9 \%$ and $8.56 \%$ respectively), whilst incorporation into succinate showed no significant change (figure 5.3 C). Despite the changes in ${ }^{13} \mathrm{C}_{6}$ glucose incorporation outlined above, the total amount of each metabolite measured by mass spectrometry showed that only lactate (increased) and itaconate (decreased) showed significantly altered steady state levels in itolDC compared to untreated iDC (figure 5.3 D).

To determine the wider metabolic implications of increased utilisation of glucose carbons by itolDC, further tracer metabolite analyses were carried out using ${ }^{13} \mathrm{C}_{5}$-glutamine (figure 5.4 A). Data indicate that there was significant incorporation of ${ }^{13} \mathrm{C}_{5}$-glutamine in $\mathrm{M}+1$ malate and aspartate, and $\mathrm{M}+5$ glutamate (figure 5.4 B). Nevertheless, overall the effects of $1,25(\mathrm{OH})_{2} \mathrm{D} 3$ on glutamine metabolism was less pronounced than with glucose, indicating that $1,25(\mathrm{OH})_{2} \mathrm{D} 3$ specifically modulates glucose entry to the TCA cycle, rather than altering the TCA cycle as a whole. These data overall also supported a hypothesis that other possible carbon sources into the TCA cycle, which include amino acids and fatty acids, were significantly reduced. 
A.

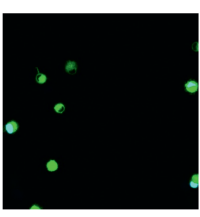

iDC

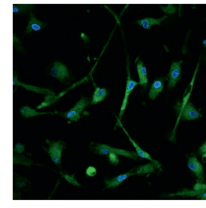

itolDC

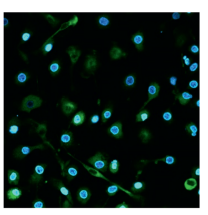

itolDC + 10uM C75

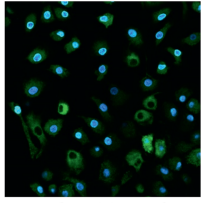

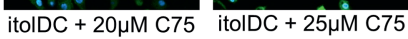

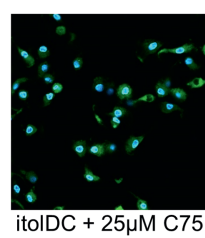

HLA-DR

B.

C.

CD11c

CD14

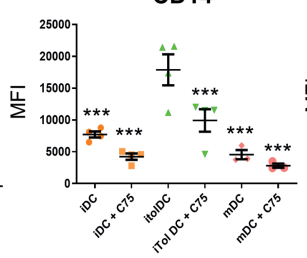

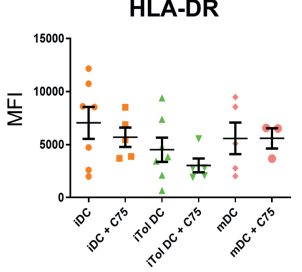

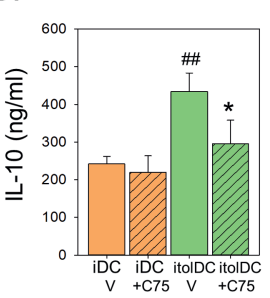

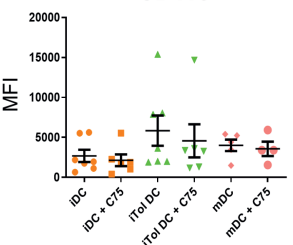

CD80

CD86
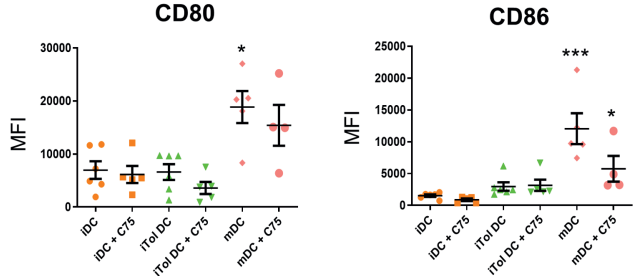

Fig. 5.6 Inhibition of fatty acid synthesis suppresses effects of $1,25(\mathrm{OH})_{2} \mathrm{D} 3$ on CD14 and IL-10 in itolDC. A. Mitotracker and DAPI immunofluorescence in iDC and $1,25(\mathrm{OH})_{2} \mathrm{D} 3$-induced itolDC, and itolDC treated with the fatty acid synthase inhibitor C75 (10-25 $\mu \mathrm{M})$. B Concentration of IL-10 in supernatants from iDC and $1,25(\mathrm{OH})_{2} \mathrm{D} 3$-induced itolDC cultured in the absence (vehicle, $\left.\mathrm{V}\right)$ or presence of $\mathrm{C} 75(20 \mu \mathrm{M})$. C flow cytometric analysis of CD14, HLA-DR, CD80 and CD86 in iDC and 1,25(OH) 2 D3-induced itolDC cultured in the absence (vehicle, V) or presence of C75 $(20 \mu \mathrm{M})$. Data are shown as frequency of expression for analysis of $\mathrm{n}=4-5$ separate donor PBMC preparations. $*=$ statistically different as indicated by bars, $\mathrm{p}<0.05$, ** $\mathrm{p}<0.01$, $* * * \mathrm{p}<0.001$. 


\subsubsection{1,25( $(\mathrm{OH})_{2} \mathrm{D}$-induces fatty acid synthesis in itolDC}

Data in figure 5.3 and figure 5.4 show the effect of $1,25(\mathrm{OH})_{2} \mathrm{D} 3$ on polar, hydrophilic metabolites derived from ${ }^{13} \mathrm{C}_{6}$-glucose and ${ }^{13} \mathrm{C}_{5}$-glutamine. To assess possible effects of $1,25(\mathrm{OH})_{2} \mathrm{D} 3$ on hydrophobic metabolites, further analysis of the non-polar fraction of cell extracts after incubation with ${ }^{13} \mathrm{C}_{6}$-glucose was carried out (figure 5.5). The pathway from TCA cycle (citrate) to fatty acids is shown in figure $5.5 \mathrm{~A}$. The relative abundance of different fatty acid isotopomers (figure 5.5 B) showed significant incorporation of ${ }^{13} \mathrm{C}_{6}$-glucose for only palmitate. However, analysis of the total pool of different fatty acids in iDC and itolDC (figure 5.5 C) showed that both palmitate and palmitoleate were increased significantly in $1,25(\mathrm{OH})_{2} \mathrm{D} 3$-induced itolDC. Collectively these data indicate that induction of itolDC by $1,25(\mathrm{OH})_{2} \mathrm{D} 3$ is associated with a significant increase in de novo fatty acid synthesis via glucose and citrate.

\subsubsection{Inhibition of fatty acid synthesis suppresses itolDC induction by $1,25(\mathrm{OH})_{2} \mathrm{D3}$}

The functional significance of fatty acid synthesis in $1,25(\mathrm{OH})_{2} \mathrm{D} 3$-induced itolDC was assessed using the fatty acid synthase inhibitor C75 [199]. C75 inhibits in an irreversible manner the FAS activity by competing with its natural substrates (acetyl-CoA, malonyl-CoA and NADPH) interfering with multiple FAS's sites including key domains: $\beta$-ketoacyl synthase, the enoyl reductase and the thioesterase [73][202]. Immunofluorescence showed that, relative to the more rounded morphology of vehicle-treated iDC, treatment with $1,25(\mathrm{OH})_{2} \mathrm{D} 3$ produced a pronounced dendritic morphology in itolDC. This effect was abrogated in itolDC treated with $\mathrm{C} 75$ which exhibited fewer dendrites and a more rounded morphology (figure 5.6 A). Analysis of DC culture supernatants also showed that inhibition of fatty acid synthase by $\mathrm{C} 75$ suppressed IL-10 production by itolDC (figure 5.6 B). Flow cytometry showed that C75 had no effect on HLA-DR, CD80 or CD86 expression by iDC, itolDC or mDC, but was associated with significant suppression of $1,25(\mathrm{OH})_{2} \mathrm{D} 3$-induced CD14 expression by itolDC (figure 5.6 B).

\subsection{Discussion}

Regulation of cellular metabolism plays a critical role in immune function, promoting changes in energy production and biosynthesis that underpin key innate immune responses to infection and tissue damage, while providing the metabolic basis for expansion of lymphocyte function in adaptive immune responses [175]. Antigen presenting cells such as macrophages and DC 
are particularly dependent on the regulation of metabolic pathways to integrate antibacterial responses and $\mathrm{T}$ cell activation, with tolerogenic attenuation of immune function to limit tissue damage [176]. Induction of glucose consumption and glycolysis plays a crucial role in the activation of DCs and their ability to acquire, process and present antigen [262]. Notably, inhibition of hexokinase, the first enzymatic step in glycolysis, potently blocks DC activation [262]. At least part of the glycolytic metabolic reprogramming associated with DC activation involves the production of lactate, with recent studies showing that lactate acts as a crucial signalling molecule for tolDC in the generation of regulatory T cells (Treg) [151]. However, DC tolerogenicity is also strongly influenced by lipid metabolism, with elevated glycolysis and electron transport being linked to increased fatty acid oxidation (FAO) in tolDC [149]. Previous studies by ourselves and others have shown that the tolerogenic effects of vitamin D on DCs are fundamentally associated with metabolic reprogramming in the form of increased glycolysis, oxidative phosphorylation and TCA cycle activity [72] [81]. To date these metabolic analyses have focused on the ability of active vitamin $\mathrm{D}, 1,25(\mathrm{OH})_{2} \mathrm{D} 3$, to modify tolerogenesis in DCs activated with immunogens such as LPS. In data presented here we show that treatment of DC with $1,25(\mathrm{OH})_{2} \mathrm{D} 3$ alone is sufficient to generate itolDC that exhibit significant metabolic reprogramming independent of immune activation, with enhanced fatty acid synthesis playing a central role in the generation of the itolDC phenotype. It would be interesting to further explore how vitamin D modulate fatty acid metabolism in tolDC that have been stimulated with LPS to determine if there are any differences at the metabolic level.

Phenotype analysis of tolerogenic DC generated with $1,25(\mathrm{OH})_{2} \mathrm{D} 3$ alone (itolDC) or with LPS and $1,25(\mathrm{OH})_{2} \mathrm{D} 3$ (mtolDC) showed significant similarities between these two types of tolerogenic DC. Both showed suppression of the DC activation markers CD209, CD86 and $\mathrm{CD} 83$ relative to $\mathrm{mDC}$, consistent with established tolerogenic responses to $1,25(\mathrm{OH})_{2} \mathrm{D} 3$ [101]. ItolDC and mtolDC also showed $1,25(\mathrm{OH})_{2} \mathrm{D} 3$-stimulated expression of CD14 and CYP24A1, again consistent with established responses to $1,25(\mathrm{OH})_{2} \mathrm{D} 3$ [101] [18]. However, itolDC and mtolDC were also phenotypically distinct, with the latter showing elevated HLADR and CD80, and increased expression of VDR and CYP27B1 consistent with established responses to immunogens such as LPS [101] study are therefore are phenotypically similar to previously reported tolDC generated using combined treatment with $1,25(\mathrm{OH})_{2} \mathrm{D} 3(100$ $\mathrm{nM}$ ) and dexamethasone (DEX) [149]. In this case, 1,25(OH) ${ }_{2} \mathrm{D} 3 / \mathrm{DEX}$-tolDC showed similar glycolytic activity to LPS-induced mDC but glycolytic reserve, respiratory capacity and metabolic plasticity were significantly higher in tolDC . Increased glycolysis and oxidative phosphorylation have also been described for tolDC induced by $1,25(\mathrm{OH})_{2} \mathrm{D} 3$ in the absence of DEX. However, in this case 6 day treatment with $1,25(\mathrm{OH})_{2} \mathrm{D} 3$ to generate 
tolDC was accompanied by activation with LPS for the final 24 hrs, thus generating an mtolDC phenotype [72]. Nevertheless, this study showed that the metabolic reprogramming induced by $1,25(\mathrm{OH})_{2} \mathrm{D} 3$ in combination with LPS was distinct from that observed for other tolerogenic treatments such as DEX+LPS [72]. Specifically, $1,25(\mathrm{OH})_{2} \mathrm{D} 3$ was shown to promote metabolic reprogramming via activation of PI3kinase-Akt-mTOR pathways, with inhibition of these pathways acting to inhibit the tolerogenic effects of $1,25(\mathrm{OH})_{2} \mathrm{D} 3$ on DC. By contrast, the effects of other promoters of DC tolerogenesis such as DEX and IL-10 were unaffected by manipulation of the PI3kinase-Akt-mTOR pathway [72]. These observations, in combination with the data present in the current study, indicate that $1,25(\mathrm{OH})_{2} \mathrm{D} 3$ alone is sufficient to promote the metabolic reprogramming required for a tolerogenic DC phenotype.

In addition to enhanced aerobic glycolysis and lactate production, $1,25(\mathrm{OH})_{2} \mathrm{D} 3+\mathrm{LPS}$ induced mtolDC have also been shown to exhibit increased TCA cycle activity. Tracer metabolite analysis using ${ }^{13} \mathrm{C}_{6}$-glucose has shown that $1,25(\mathrm{OH})_{2} \mathrm{D} 3$ promotes ${ }^{13} \mathrm{C}$-labeling of several TCA metabolites, consistent with increased incorporation of glucose into the TCA cycle [243]. In the current study we observed a similar significant increase in TCA cycle flux in itolDC induced by $1,25(\mathrm{OH})_{2} \mathrm{D} 3$ alone. Importantly, by using both ${ }^{13} \mathrm{C}_{6}$-glucose and ${ }^{13} \mathrm{C}_{5}$-glutamine as TCA cycle substrates, we were able to confirm that the metabolic reprogramming actions of $1,25(\mathrm{OH})_{2} \mathrm{D} 3$ are focused primarily on strongly enhanced incorporation of glucose into lactate and the TCA cycle (figure 5.4 B and C). Nevertheless, in general the overall concentrations of individual TCA metabolites were not significantly altered in itolDC (figure 5.3 D). This suggests that these metabolites are highly incorporated into other metabolic pathways in DC treated with $1,25(\mathrm{OH})_{2} \mathrm{D} 3$. One example of this is the diversion of aconitate away from the TCA cycle to generate itaconate. Interestingly, itaconate was the only TCA metabolite that showed lower steady state levels in $1,25(\mathrm{OH})_{2} \mathrm{D} 3$-induced itolDC. In inflammatory macrophages itaconate has been shown to be elevated, but its function appears to be as part of an anti-inflammatory feedback mechanism in these cells by inhibiting NF- $\chi \mathrm{B}$ activity [163]. Itaconate has also been shown to exhibit antibacterial properties [146]. Given that itaconate fulfils the same dual antibacterial [97] [99] and anti-inflammatory function [99] [3] attributed to $1,25(\mathrm{OH})_{2} \mathrm{D} 3$, it seems paradoxical that $1,25(\mathrm{OH})_{2} \mathrm{D} 3$-induced itolDC should show decreased levels of itaconate. A possible explanation for this is that the increased use of TCA cyle products in fatty acid synthesis by itolDC may divert carbons away from itaconate synthesis, which in turn decreases the suppressive function of itaconate on TCA cycle activity [138]. The resulting increase in TCA cycle activity may therefore be central to the ability of $1,25(\mathrm{OH})_{2} \mathrm{D} 3$ to promote a tolerogenic DC phenotype, with $1,25(\mathrm{OH})_{2} \mathrm{D} 3$ activating separate, but equally effective, antibacterial and anti-inflammatory mechanisms 
via direct non-metabolic remodelling mechanisms.

Both glycolysis and the TCA cycle can contribute to further metabolic pathways such as the proteins and lipid synthesis. In the current study we have shown for the first time that metabolic reprogramming effects of $1,25(\mathrm{OH})_{2} \mathrm{D} 3$ on glucose consumption, glycolysis and TCA cycle activity in DC are associated with increased generation of fatty acids. Fatty acid synthesis appears to play a pivotal role in the generation and survival of DCs, with inhibition of FAS decreasing DC development but also enhancing T cell activation by DC, with these effects being mediated partly through increased ER stress [201]. In a similar fashion, exogenously added polyunsaturated fatty acids have been shown to block $\mathrm{T}$ cell activation by DC [266]. It is important to recognise the effects of fatty acids on DC function may be dependent on the context of DC development, as hepatic DC with low levels of fatty acids showed a tolerogenic phenotype and promoted Treg generation [107]. Palmitate and palimoleate are fatty acid precursors but they may also themselves exert anti-inflammatory effects, with palmitoleate functioning as a lipokine to regulate AMP kinase [39], and suppress inflammation [48]. Collectively these observations indicate that the generation of palmitate and palmitoleate from glucose may be a pivotal component of the metabolic function of $1,25(\mathrm{OH})_{2} \mathrm{D} 3$ in DC. It was therefore interesting to note that although inhibition of FAS in itolDC resulted in profound changes in DC morphology, effects on DC phenotype were less pronounced. Consistent with the itolDC IL-10 analyses presented here, previous studies of inflammatory macrophages have shown that inhibition of FAS significantly suppresses expression of several cytokine markers, including IL-10 [38]. Given the fundamental importance of IL-10 for DC tolerogenesis [60] it is clear that FAS may play a key role in the ability of $1,25(\mathrm{OH})_{2} \mathrm{D} 3$ to promote tolerogenic DC. The role of CD14 as a marker of $1,25(\mathrm{OH})_{2} \mathrm{D} 3$-induced metabolic reprogramming is less clear and may simply reflect the elevated levels of CD14 in itolDC, moreover a trend towards suppression of CD14 with FAS inhibition for iDC and $\mathrm{mDC}$ as well as itolDC.

Data presented in this study underline the importance of $1,25(\mathrm{OH})_{2} \mathrm{D} 3$ as a potent stimulator of metabolic reprogramming in DC. In contrast to previous studies where the effects of $1,25(\mathrm{OH})_{2} \mathrm{D} 3$ were assessed in conjunction with DC activation by immunogenic stimuli, we show here that $1,25(\mathrm{OH})_{2} \mathrm{D} 3$ alone is sufficient to reprogram metabolism and generate itolDC. In particular, the tolerogenic response to $1,25(\mathrm{OH})_{2} \mathrm{D} 3$ was associated with increased glycolysis and lactate generation, and enhanced TCA cycling leading to fatty acid synthesis. Inhibition of fatty acid synthesis was sufficient to alter the morphological and IL-10 induction effects of $1,25(\mathrm{OH})_{2} \mathrm{D} 3$, indicating that this component of metabolism if crucial for 
tolerogenesis. However, it is likely that $1,25(\mathrm{OH})_{2} \mathrm{D} 3$ coordinates a spectrum of changes in DC metabolism, notably including the potent generation of lactate in itolDC. Recent studies have shown the lactate itself is a pivotal DC-metabolite that promotes the generation of Treg [151], so that the induction of itolDC by $1,25(\mathrm{OH})_{2} \mathrm{D} 3$ may involve altered DC morphology and function via enhanced fatty acid synthesis, as well as indirect effects on $\mathrm{T}$ cells via lactate generation. 



\section{Chapter 6}

\section{General discussion}

\subsection{General discussion}

This thesis demonstrates how joint efforts from dry and wet international laboratory research groups can integrate different research strategies to perform multidimensional data analysis, and shed new lights on molecular interactions within specific biological systems. In the current project, both dry and wet laboratory studies gave a new perspective on the molecular mechanisms by which vitamin D interacts with extra-skeletal target tissues such as lymphoid and myeloid models and, in particular, highlighting novel pathways for the immunomodulatory actions of $1,25(\mathrm{OH})_{2} \mathrm{D} 3$.

\subsection{Bioinformatic pipelines for functional omics data anal- ysis}

In the modern era of bioscience research, publishing results in a journal is not the endpoint of scientific work. Data generated from experiments can be shared in open data repositories from where other researchers can retrieve original raw data generated from wet-lab work. This provides the opportunity to apply different bioinformatic approaches to perform i) in-depth, follow-up analyses of the original data or ii) an integrative data analysis combining multiple datasets. Nowadays, sharing of data is extended with the FAIR principles which define that data should be findable, accessible, interoperable and reusable [259]. These principles will allow more complete and better integration of different dataset in the future. In the end, this will ease the access to experimental datasets and usage of tools that will result in optimal interrogation of research data at different levels, optimizing the generation of research outputs and, in consequence, guaranteeing better understanding of biological 
concepts seen from different perspectives. The objective of this $\mathrm{PhD}$ project was to exploit and compare publically available data by the application of different bioinformatic tools in the same $\mathrm{PhD}$ project for the purpose of contextualizing it at different biological levels, and then design and execute tailored-made wet-lab experiments to further analysis of the original datasets. The resulting thesis, therefore, illustrates how bioinformatics strategies can be incorporated into conventional laboratory research to more rapidly moving research ideas forward.

\subsubsection{Discovery of new molecular aspects of vitamin $D$ by exploiting public gene expression datasets}

A major research advance provided by the bioinformatics strategy used in this project was to improve the visualisation of $1,25(\mathrm{OH})_{2} \mathrm{D} 3$ target responses. Conventional gene ontology analysis of differentially expressed genes provides a very crude overview of the cellular processes that may be regulated by a particular treatment or condition. In the current thesis the aim was to use a much more specific and organised approach via pathway analysis that analyses gene expression using established, curated, biological systems to study the role of $1,25(\mathrm{OH})_{2} \mathrm{D} 3$ in immunoregulation. Analysing gene expression data in a pathway context reveals how specific conditions or factors affect a particular biological system in a particular cell/tissue model. The workflow for this analysis includes PathVisio, a freely-available and open-source tool that performs data analysis at the pathway level revealing altered biological routes within a specific system or treatment. PathVisio [136] performs this pathway analysis using pathway repositories such as WikiPathways [221], a pathway database which is constantly curated and updated by the scientific community to enable data visualization in biological pathways. This kind of diagrammatic representation enhances the contextualization of experimental data into biological processes, allowing a better understanding of how a system works.

The application of the different bioinformatic tools compiled in the pipeline using published data that is described in Chapter 2 allowed us to analyse how the active form of vitamin $\mathrm{D}, 1,25(\mathrm{OH})_{2} \mathrm{D} 3$, modulates gene expression in different cell models including $\mathrm{NK}$ cells (Chapter 3), monocytes, THP-1 cell line, DC, and lymphocytes (Chapter 4) and to perform a statistical analysis that contextualizes these gene expression changes at the pathway level. The results of this statistical approach gave us a new perspective of how $1,25(\mathrm{OH})_{2} \mathrm{D} 3$ is involved in crucial cellular processes such as metabolism, proliferation, differentiation and signaling. In particular, we demonstrated how $1,25(\mathrm{OH})_{2} \mathrm{D} 3$ has a significant impact on immune cell metabolism, suggesting that the $1,25(\mathrm{OH})_{2} \mathrm{D} 3$ is a key factor for metabolism 
reprogramming in tolerogenic dendritic cells. The conclusions from this dry-lab approach (Chapter 4) were used as a basis for the the hypothesis in the wet-lab part of the project (Chapter 5), where, we explored the molecular effects of $1,25(\mathrm{OH})_{2} \mathrm{D} 3$ on metabolism in dendritic cells. However, the rapidly increasing availability of 'omics' data means that bioinformatics strategies such as pathway analysis are now frequently incorporated into mainstream research publications. The publication of datasets from genomic, proteomic and metabolomic studies allows the integration of different types of biological data into an integrative analysis that allows a better understanding of a specific biological system.

Finally, we propose that a next step to this work would be to characterise DC in vivo by isolating them from blood samples. One interesting set of experiments would be to obtain these samples from vitamin D deficiency patients and healthy control ones. Cells will be specifically sorted and selected by FACS to next perform a single cell RNA-seq assay. This study will show the most characteristic DC populations in both cases and, most importantly, will help to understand how vitamin D deficiency affects DC development in vivo. Furthermore, with this technique we will be able to cluster cells in groups based in their gene expression profiles giving the possibility to compare DC sub-populations. Finally, these results can be integrated in the bioinformatic workflow described in this thesis and contextualize these gene expression differences at the pathway level and highlight those metabolic and signalling pathways affected in specific DC sub-populations.

\subsubsection{Understanding biological systems using integration of omics data}

In bioscience research, wet-lab experiments analyse molecular dynamics within biological systems through the utilization of laboratory equipment that measures changes in parameters that indicate alterations in affected molecules that are the subject of a particular study. Using this approach it is possible to show how specific conditions can modulate gene or protein expression, metabolic fluxes, molecule-molecule interactions, molecular dynamics and other biological aspects within a specific tissue or cell. With the evolution of bioscience research, new techniques and methodologies have become available that can perform large scale analyses that can generate large experimental datasets. Such 'omics' approaches have become increasingly convenient and financially accessible, and they greatly expand the scope of research projects. However, the major challenge with large datasets is the interpretation and contextualization of data. A key objective of the current $\mathrm{PhD}$ was to investigate improved strategies for interpretation and contextualization of gene expression datasets for studies of immune responses to vitamin $\mathrm{D}$. 
Cytoscape [223] allows the integration of various molecule-molecule interactions by using external interaction biological databases (transcription factors, miRNA, drugs, proteins, etc). The app CyTargetLinker allows the integration of data in and automated manner into Cytoscape, extending the biological network with additional molecule-molecule interactions to create a biological network representing the different interactions of molecules that are part of that system. When used with Cytoscape, Apps such as CyTargetLinker also allow data analysis and visualization, to better understand the dynamic basis of a specific biological network. Open bioinformatic tools can even go further and exploit omics data at a different biological level that were not considered in previous analyses and add additional information to the biological interpretation. Both PathVisio and Cytoscape, allow the integration of multiple sets of omics data, including data from metabolic, proteomic and even flux reactions studies. The combination of different types of data greatly helps to improve our understanding of biology systems by providing the widest possible representation of molecular interactions and dynamics by pathway and network for any particular dataset. Further to our findings regarding the positive effect of vitamin D on glycolysis, ETC and TCA cycle in tolerogenic DC, more metabolic analysis is needed to identify how vitamin D modulates these pathways, in order words how the active form of vitamin D affects metabolic fluxes. With the bioinformatic tools described in this thesis, is also possible to incorporate data from metabolomic studies and merge these with established gene expression data to have a better biological interpretation of the role of vitamin D in immunometabolism.

\subsubsection{Automatization and integration of open bioinformatic tools}

Effective use of bioinformatic tools requires comprehensive and easy access guidelines. This is particularly important when several interactive softwares are brought together in a workflow. In this thesis, Chapter 2 contains the description and instructions for the bioinformatic pipeline used in the dry-lab part of this $\mathrm{PhD}$ project. The availability of a well documented dry-lab pipeline eases the automatisation of open bioinformatic platforms. For instance, Cytoscape [179] and Pathvisio [24] are two open source bioinformatic tools that can also be used through remote programming interfaces and these automation packages are well documented allowing their usage in basic programming languages such as Python and $\mathrm{R}$.

The major benefit of the fact that these tools are well documented and available for the most common coding languages helps to integrate and automate these tools in a single pipeline. This could be done by the integration of the different scripts and modules to use the different tools in a common language, or for instance in a jupyter notebook, in order to combine their functionality. Furthermore, this integration can be done in an open friendly- 
user platform such as Galaxy [6] where different tools can be put together to generate an open workflow which can analyse all kinds of omics data. The incorporation of part of the analytical tools of PathVisio and Cytoscape in a Galaxy workflow will ease their accessibility and this type of omics data analyses will become more reproducible.

\subsection{Vitamin D and immunometabolism}

\subsection{1 $1,25(\mathrm{OH})_{2} \mathrm{D} 3$ modulates immune cell function which is accompa- nied by a metabolic reprogramming}

Data from Chapter 4 of this thesis indicate that vitamin D promotes metabolic reprogramming in a variety of myeloid cell types and not just DC. Thus, in future studies it will be interesting to determine the role of metabolism in other aspects of vitamin D immunoregulation. In particular, vitamin $\mathrm{D}$ is known to be a potent inducer of antibacterial responses in macrophages [14]. Although vitamin D can induce antibacterial proteins [98], promote autophagy [106], and modulate intracellular iron concentrations [15] to stimulate antimicrobial responses to infection, it would be interesting to investigate a role for metabolic reprogramming in this part of innate immune function. Previous studies have linked metabolic pathways to antimicrobial responses. For instance in APC, such as M1 macrophages and activated DC, glycolysis plays an important role in some specific cell functions such as motility, inflammatory cytokine production, ROS production and pathogen processing phagocytosis [76][162][185][90] and, as we have seen that vitamin D impact these pathways (Chapter 4 [81]), we can hypothesize that vitamin D could modulate immune responses through metabolic reprogramming.

Finally, although the data in this thesis suggested that vitamin D was not a major regulator of metabolic pathways in lymphocytes relative to myeloid cells, we have to acknowledge that this analysis was restricted to B cells. In future studies it will be interesting to determine if $1,25(\mathrm{OH})_{2} \mathrm{D} 3$ also has metabolic reprogramming effects on $\mathrm{T}$ cells that are independent of its actions on DC. Proliferating T cells express the VDR and can respond to $1,25(\mathrm{OH})_{2} \mathrm{D} 3$ in the absence of DC by promoting anti-inflammatory, pro-tolerogenic $\mathrm{T}$ cell responses [110] [111]. Therefore, further experiments should determine if $1,25(\mathrm{OH})_{2} \mathrm{D} 3$ regulates $\mathrm{T}$ cell metabolism in the same way that it stimulates myeloid and DC metabolism. 


\subsection{Therapeutic applications}

Our findings serve primarily as a molecular overview of how vitamin D promotes a tolerogenic phenotype in DC and how this development is accompanied by the establishment of a specific metabolic cell profile. The identification of the positive modulation of fatty acid synthesis by the hormone in this tolerogenic dendritic cells, brings new light to future research work aimed at investigating how the modulation of specific metabolic routes directs tolerogenic immune cell function. Finding key metabolic targets will help to optimize cell culture conditions for the generation of tolerogenic dendritic cells that promote $\mathrm{T}$ reg and inhibit proinflammatory $\mathrm{T}$ cells which are key aspects for autoimmune diseases [74]. Understanding how these metabolic pathways function may also help to identify new targets for therapy in autoimmune diseases, but could also help to improve the efficacy of agents such as vitamin $\mathrm{D}$ that have known tolerogenic properties. The therapeutic use of vitamin $\mathrm{D}$ or vitamin D metabolites for extra-skeletal disorders remains controversial, and several recent vitamin D supplementation studies in humans in vivo have failed to show significant health benefits [194][119][144]. However, it is possible that $1,25(\mathrm{OH})_{2} \mathrm{D} 3$ could provide therapeutic benefits if used ex vivo. Studies have shown that $1,25(\mathrm{OH})_{2} \mathrm{D} 3$-induced tolerogenic DC have potential therapeutic benefits for regulating $\mathrm{T}$ cell function in inflammatory diseases such as rheumatoid arthritis [11] and host-graft rejection in transplantation [243]. Consequently, it will be interesting to determine if metabolic pathways and factors identified in the current project can improve the use of vitamin D in this type of ex vivo strategy.

Data from the current $\mathrm{PhD}$ have also highlighted further experiments that could be carried out to extend the project. The most obvious of these future studies is to assess the functional impact of fatty acid synthesis on the ability of $1,25(\mathrm{OH})_{2} \mathrm{D} 3$ to promote $\mathrm{T}$ cell responses as a consequence of its effects in stimulating tolerogenic DC generation. T cell-DC co-culture experiments in the presence or absence of a fatty acid synthase inhibitor would allow us to determine whether the enhanced fatty acid synthesis in $1,25(\mathrm{OH})_{2} \mathrm{D} 3$-induced tolerogenic DC is essential for the generation of Treg by vitamin D previously described by the Birmingham group [113]. These experiments could include inhibition of fatty acid synthesis in T cells and DC separately, or together, to define the precise way in which metabolic reprogramming can influence antigen presentation and $\mathrm{T}$ cell phenotype. In addition to using inhibitors of fatty acid synthesis, future studies should also include the use of inhibitors of other stages of metabolism. Previous studies have explored the DC function using low glucose culture conditions to reduce glycolysis [72], as well as inhibitors of the glycolytic enzyme 6-phosphofructo-2-kinase/fructose-2,6-biphosphatase 4 (PFKFB4) as a key step in glycolysis [243]. However, data from this PhD project suggest that the functional impact 
of $1,25(\mathrm{OH})_{2} \mathrm{D} 3$ responses in DC is further downstream of glycolysis. Thus, as well as exploring the effects of fatty acid synthesis inhibitors on DC-T cell interactions, future studies should also investigate the possible effects of the products of DC fatty acid synthesis. It has been hypothesized that fatty acid molecules such as palmitoleic acid can act as lipokines that could be used as a non pharmacological drugs to prevent inflammatory diseases [53]. Future studies should also determine if exogenous addition of fatty acids is sufficient to alter DC or $\mathrm{T}$ cell phenotype, and how this could impact inflammatory disease.

\subsection{Vitamin D supplementation in personalized medicine}

There has been a constant debate about the adequate daily dietary intake of vitamin D to prevent vitamin D deficiency. Despite a few differences, according to both the Institute of Medicine (IOM) [211] and the Endocrine Society [104] it is suggested that during infancy and childhood a intake of 400-600 IU/day is recommended. Furthermore, the Endocrine Society suggests that adults require 1500-2000 IU/day while the IOM only recommends increasing the dietary intake only to adults over 70 years to $800 \mathrm{IU} /$ day [69]. As it is discussed in Chapter 57 of the "Vitamin D: health, disease and therapeutics", these disagreements are explained based on the target population that both societies aimed to advise [69]. This debate goes further when vitamin $\mathrm{D}$ is suggested as a therapeutic treatment (or to prevent) for specific diseases. So far, vitamin D has been suggested to be a positive factor to prevent or treat diseases related to falls related to muscle and bone weakness [23], autoimmune [225], metabolic disorders [234] and leukemia [218]. Despite the fact that these studies suggest that vitamin $\mathrm{D}$ might be a beneficial factor to treat or prevent certain diseases, other studies also prove that no significant positive effect has been seen with vitamin D as a natural therapeutic option [194][119][144].

Although the current $\mathrm{PhD}$ is focused on the possible role of vitamin $\mathrm{D}$ as a metabolic regulator in immune cells during normal responses to immune stimuli, it is possible that these metabolic responses are altered in disease states. In particular this may be important for autoimmune diseases such as type 1 diabetes, multiple sclerosis and inflammatory bowel disease as previously described. However, the data from this thesis also highlight another facet of vitamin D-induced regulation of immune cells. Specifically, in monocytic cell lines derived from patients with leukemia, the transcriptomic effects of vitamin D were distinct from its effects on normal immune cells. Notably $1,25(\mathrm{OH})_{2} \mathrm{D} 3$ induced potent effects on genes associated with cell proliferation and cell cycle progression as described in Chapter

4. This is consistent with studies carried out may years ago suggesting that $1,25(\mathrm{OH})_{2} \mathrm{D} 3$ 
is a potent suppressor of leukaemic cells. However, to date it is not clear yet the beneficial use of $1,25(\mathrm{OH})_{2} \mathrm{D} 3$ to treat leukemia, since many preclinical trials have shown detrimental calcium-related side-effects in humans [157].

This contrast in the effects of vitamin D in health reflects that further molecular research is needed to understand how vitamin $\mathrm{D}$ can be beneficial for therapeutic treatment and disease prevention and how intrinsic and extrinsic factors may influence how patients respond to vitamin D supplementation. For instance, since genetic variations are linked to diseases and treatment response, observational approaches such as Genome-Wide Association Studies (GWAS) are performed to understand how single genetic variations, also known as singlenucleotide polymorphism (SNPs), among the genome are linked to individual phenotypes. In this particular matter, GWAS studies have demonstrated that vitamin D metabolism relies on some gene variations [114], adding another layer of complexity for the research of the usage of vitamin $\mathrm{D}$ as therapeutic alternative to treat and prevent diseases.

\subsection{Final words}

Bioscience research aims to understand biological systems to achieve one of the biggest efforts of modern science: improve health quality. Specific fields of bioscience contribute to decipher biological questions through the performance of experimental work which generates results that serve a single puzzle piece of a whole big picture of a specific biological system. Collaborative work between different fields of sciences promotes the elaboration of a scientific network that connects distinct methodologies allowing different approaches to a specific biological question. In this particular case, thanks to the efforts of two distinct research groups with different scientific backgrounds we have brought together complementary methodologies to shed new light on the role of vitamin D in immunometabolism. 


\section{Summary}

This thesis is the result of a joint collaboration between research institutes with expertise in two distinct aspects of science: cell biology and bioinformatics. By using state of the art techniques from both of these fields the thesis describes the discovery of an entirely new role for vitamin $\mathrm{D}$ in the immune system, as a potent regulator of immunometabolism. To demonstrate this, the project initially focused on bioinformatic analyses in which we developed applied computational techniques to interrogate publically available transcriptomic datasets generated in studies of the immunomodulatory effects of the active form of vitamin D, 1,25-dihydroxyvitamin D3 $\left(1,25(\mathrm{OH})_{2} \mathrm{D} 3\right)$. Using this bioinformatic approach we were able to demonstrate that the predominant effect of $1,25(\mathrm{OH})_{2} \mathrm{D} 3$ on myeloid derived innate immune cells such as monocytes and dendritic cells (DC) was to regulate cell metabolism via enhanced glycolysis, electron transport, oxidative phosphorylation and TCA cycle activity. In the second half of this thesis, we have explored the immunometabolic function of $1,25(\mathrm{OH})_{2} \mathrm{D} 3$ further through in vitro experiments that investigated the specific function of immunometabolism in mediating the tolerogenic effects of $1,25(\mathrm{OH})_{2} \mathrm{D} 3$ on DC. The overall conclusion from these cellular experiments was that the ability of $1,25(\mathrm{OH})_{2} \mathrm{D} 3$ to promote anti-inflammatory tolerogenic innate immune responses by DC is crucially dependent on the regulation of cell metabolism rather than specific immune actions. This new perspective on the immunomodulatory actions of vitamin D may help to identify new strategies for the therapeutic use of vitamin D in the treatment of inflammatory disease.

\section{Contextualizing public gene expression data into biological pathways}

As mentioned above, the first part of this $\mathrm{PhD}$ project focused on the generation of a bioinformatic workflow or 'pipeline' that was used to retrieve and analyse large gene expression datasets for different immune cell models that were treated with the active form of vitamin $\mathrm{D}$, $1,25(\mathrm{OH})_{2} \mathrm{D} 3$. Gene expression datasets were initially retrieved from public repositories in as raw experimental data. As described in Chapter 2, we established a bioinformatic pipeline that analysed the quality of the raw expression data and processed these data to retrieve gene 
expression parameters that enabled the contextualization of the data at functional pathway level using the PathVisio and WikiPathways open-source bioinformatics platforms. Furthermore, the integration of another open-source software platform, Cytoscape, into the pipeline enabled the use of different apps that allowed integration of additional biological information into the analysis to generate biological networks that plot molecule-molecule interactions. Cytoscape also enabled visualization of experimental data resulting in a user-friendly way to understand biological activities that were occurring in response to treatment with vitamin $\mathrm{D}$.

Once the PathVisio/WikiPathways/Cytoscape pipeline was established, the next aim of the project was to test the pipeline using different lymphoid cell models that had been used to investigate the actions of $1,25(\mathrm{OH})_{2} \mathrm{D} 3$ (Chapter 3). The bioinformatic pipeline was applied to a vitamin D study that had generated RNA-seq transcriptomic data in a wet-lab study, but where the overall impact of $1,25(\mathrm{OH})_{2} \mathrm{D} 3$ was still unclear. The aim here was to incorporate the PathVisio/WikiPathways/Cytoscape pipeline into an existing study to provide a new perspective on the available transcriptomic data. J A Tamblyn et al. [236] explored the immunomodulatory role of $1,25(\mathrm{OH})_{2} \mathrm{D} 3$ in two different natural killer $(\mathrm{NK})$ cell populations: uterine NK ( $\mathrm{uNK}$ ) from the decidual tissue of the placenta and peripheral blood NK ( $\mathrm{pNK}$ ). Bioinformatic analysis of activated $\mathrm{uNK}$ and $\mathrm{pNK}$ gene expression data showed that $\mathrm{uNK}$ were transcriptionally distinct from pNK despite the fact that both cells express similar cell surface markers [236]. This new information supported the central hypothesis that uNK have completely different actions in the decidua compared to pNK immune function in the general circulation. These studies also showed that the vitamin D receptor (VDR) system for responding to $1,25(\mathrm{OH})_{2} \mathrm{D} 3$ was differentially expressed in uNK relative to $\mathrm{pNK}$, but multiple comparison analysis indicated that this did not result in significant differential effects of $1,25(\mathrm{OH})_{2} \mathrm{D} 3$ on gene expression in these cells. The conclusion from this part of my $\mathrm{PhD}$ project was that the PathVisio/WikiPathways/Cytoscape pipeline developed at the start of the $\mathrm{PhD}$ project could be used successfully to clarify existing transcriptomic datasets and identify specific mechanistic targets for future studies.

After showing that the PathVisio/WikiPathways/Cytoscape pipeline could be incorporated into recently generated transcriptomic datasets from individual studies, the next stage of the project was to apply this bioinformatic strategy to analyse multiple publically available datasets. The aim of this approach was to determine if pathway analysis could provide a much broader view of the actions of $1,25(\mathrm{OH})_{2} \mathrm{D} 3$ in a biological system. In this case, the project focused on the actions of vitamin $\mathrm{D}$ as an established regulator of innate immunity. Multiple transcriptomic datasets from $1,25(\mathrm{OH})_{2} \mathrm{D} 3$-treated myeloid cell types - monocytes, 
DC, and monocytic cell lines - as well as non-myleoid lymphoid cells - were analysed using PathVisio/WikiPathways/Cytoscape. This approach showed clearly that innate immune responses to $1,25(\mathrm{OH})_{2} \mathrm{D} 3$ were associated with dramatic metabolic reprogramming. Notably enhanced glycolysis, electron transport, oxidative phosphorylation and TCA cycle activity (Chapter 4). These effects were quite distinct from other, better recognised, responses to $1,25(\mathrm{OH})_{2} \mathrm{D} 3$, such as antiproliferative pathways, that were well represented in the monocytic cell lines but not in primary cultures of monocytes or DC. Identification of novel metabolic reprogramming responses to $1,25(\mathrm{OH})_{2} \mathrm{D} 3$ in primary cultures of myeloid cells provided the platform for the final part of the $\mathrm{PhD}$ which was to use the bioinformatic data analysis to design new wet-lab experiments.

\section{Exploring the role of vitamin $D$ in immunometabolism in dendritic cells}

It is well established that vitamin $\mathrm{D}$ can regulate $\mathrm{DC}$ development in vitro to promote a more anti-inflammatory, tolerogenic DC phenotype. However, the mechanisms by which $1,25(\mathrm{OH})_{2} \mathrm{D} 3$ achieves these effects is much less clear. The aim of the final part of the $\mathrm{PhD}$ project was to address this by applying information obtained in Chapter $\mathbf{3}$ and Chapter 4 to wet-lab studies of peripheral blood mononuclear cell-derived DC. The resulting work described in Chapter 5 was carried out to investigate the hypothesis that the tolerogenic effects of $1,25(\mathrm{OH})_{2} \mathrm{D} 3$ are due to metabolic reprogramming of $\mathrm{DC}$. The novel approach used to test this hypothesis was to focus specifically on the direct effects of $1,25(\mathrm{OH})_{2} \mathrm{D} 3$ by analysing immature tolerogenic DC (itolDC) that had been exposed to $1,25(\mathrm{OH})_{2} \mathrm{D} 3$ in the absence of any other immune modulators. In this way, we aimed to assess direct responses to $1,25(\mathrm{OH})_{2} \mathrm{D} 3$ rather than the ability of $1,25(\mathrm{OH})_{2} \mathrm{D} 3$ to oppose other DC modulators such as actions of the immunogen lipopolysaccharide (LPS). Initial experiments were carried out to confirm that genes involved in electron transport chain, and tricarboxylic acid cycle were upregulated in itolDC relative to control iDC. Subsequent functional studies showed increased oxygen consumption in itolDC. This effect was observed for treatment with $1,25(\mathrm{OH})_{2} \mathrm{D} 3$ alone but not for DC treated with LPS to generate mature DC (mDC), or for DC with only short-term exposure to $1,25(\mathrm{OH})_{2} \mathrm{D} 3$ (iDC+1,25D).

Data from Chapter 3 and Chapter 4 also showed that $1,25(\mathrm{OH})_{2} \mathrm{D} 3$ promoted glucose metabolism pathways in DC. Further studies were therefore carried out using ${ }^{13} \mathrm{C}_{6}$-labeled glucose to trace the metabolism of glucose in itolDC. These tracer experiments carried out using mass spectrometry confirmed that at a functional level, glycolysis and the TCA cycle were positively regulated by $1,25(\mathrm{OH})_{2} \mathrm{D} 3$ in itolDC. Tracer studies were also carried out 
using ${ }^{13} \mathrm{C}_{5}$-labeled glutamine to determine if alternative TCA cycle substrate sources could also be enhanced by $1,25(\mathrm{OH})_{2} \mathrm{D} 3$ in itolDC. However, these experiments revealed only minor changes in TCA cycle metabolism by $1,25(\mathrm{OH})_{2} \mathrm{D} 3$, confirming that glucose metabolism is the major target for $1,25(\mathrm{OH})_{2} \mathrm{D} 3$-regulation in DC. Finally, although ${ }^{13} \mathrm{C}$ tracer studies showed potent DC metabolic reprogramming in response to $1,25(\mathrm{OH})_{2} \mathrm{D} 3$, these studies were focused on polar carbohydrate metabolites. Analysis of non-polar lipophilic metabolites of

${ }^{13} \mathrm{C}_{6}$-glucose metabolism revealed significant incorporation of label ${ }^{13} \mathrm{C}$ in palmitate, suggesting that $1,25(\mathrm{OH})_{2} \mathrm{D} 3$ promotes metabolic remodelling and fatty acid synthesis in itolDC.

Collectively wet-lab data confirmed the bioinformatic analysis of myeloid cells and specifically DC, that metabolic reprogramming is the key innate immune response to $1,25(\mathrm{OH})_{2} \mathrm{D} 3$. Furthermore, tracer analysis of glucose metabolism indicated that glycolysis and the TCA cycle were important target pathways for $1,25(\mathrm{OH})_{2} \mathrm{D} 3$, and analysis of non-polar metabolites of glucose indicated that a key consequence of DC treatment with $1,25(\mathrm{OH})_{2} \mathrm{D} 3$ was increased synthesis of fatty acids. We, therefore, proposed an additional hypothesis for wet-lab studies that synthesis of fatty acids was essential for the tolerogenic effects of $1,25(\mathrm{OH})_{2} \mathrm{D} 3$ in DC. To test this hypothesis we blocked fatty acid synthase in itolDC and studied the effect of this on DC phenotype and function. The resulting data indicated that inhibition of fatty acid synthesis altered itolDC morphology and suppressed expression of CD14 and IL-10 by these cells. These data indicate that the ability of $1,25(\mathrm{OH})_{2} \mathrm{D} 3$ to induce tolerogenic DC is dependent on metabolic remodelling leading to the synthesis of fatty acids.

\section{$\underline{\text { Conclusion }}$}

Data presented in this thesis show how improved analysis of large datasets and visualisation of these outcomes can help to identify entirely new target mechanisms for vitamin D in a cell model - DC - that had been previously well studied. Thus, the application of pathway analysis and network software tools to experimental transcriptomic datasets provided a completely new perspective on the immune actions of vitamin D. In addition, by applying the same bioinformatic pipeline to recently generated datasets from other experimental models, we were able to demonstrate the broader impact of pathway analysis in helping to clarify conclusions from diverse studies of vitamin D function. Pathway analysis also allowed us to streamline subsequent wet-lab experiments and more rapidly investigate novel hypotheses that arose from the original bioinformatic analysis. In this $\mathrm{PhD}$ project, wet-lab studies were carried out only for the last two years of the project. However, because we were able to identify more clearly target pathways, we were able to better design functional experiments. 
This is particularly important in the field of metabolism research where technologies such as tracer metabolite analysis are expensive and time-consuming. With ongoing challenges in research funding, it may be important in the future to incorporate bioinformatic analysis of datasets as a possible prelude to subsequent lab work. The overall conclusion from this $\mathrm{PhD}$ project is that metabolic reprogramming is a crucial part of the interaction between vitamin $\mathrm{D}$ and the immune system, and further investigation of this may improve the use of vitamin $\mathrm{D}$ as a possible therapy for autoimmune inflammatory diseases. 



\section{Resumen}

Esta tesis es el resultado de una colaboración conjunta entre dos grupos de investigación especializados en dos áreas científicas: biología celular y bioinformática. Gracias al uso de técnicas modernas desarrolladas en ambos campos científicos esta tesis describe el descubrimiento de un nuevo papel de la vitamina D en el sistema inmune como un potente regulador del inmuno-metabolismo. Para demostrarlo, el inicio de este proyecto se centró en realizar análisis bioinformáticos a partir de los cuales se pudieron desarrollar técnicas computacionales que permiten analizar set de datos transcriptómicos originados por experimentos centrados en investigar los efectos inmuno-moduladores de la forma activa de la vitamina D, 1-,25-dihidroxivitamina D3 $\left(1,25(\mathrm{OH})_{2} \mathrm{D} 3\right)$. El uso del conjunto de estas técnicas bioinformáticas hizo posible demostrar que el efecto predominante de $1,25(\mathrm{OH})_{2} \mathrm{D} 3$ en células inmunes innatas derivadas del linaje mieloide, tales como monocitos y células dendríticas, es regular el metabolismo celular a través de la actividad de glicólisis, cadena de transporte de electrones, fosforilación oxidativa y el ciclo de Krebs. En la segunda mitad de la tesis, exploramos en mayor detalle la función immuno-metabólica de $1,25(\mathrm{OH})_{2} \mathrm{D} 3$ por medio de la realización de experimentos in vitro, a través de los cuales investigamos la función inmuno-metabólica específica de la vitamina D por la cual modula los efectos tolergénicos en células dendríticas. Gracias a estos experimentos pudimos llegar a la conclusión de que la habilidad de $1,25(\mathrm{OH})_{2} \mathrm{D} 3$ de promover, a través de células dendríticas, respuestas tolerogénicas y anti-inflamatorias en el sistema inmune innato, es dependiente de una regulación metabólica celular frente a una regulación puramente inmunológica. Esta nueva perspectiva sobre las acciones inmuno-moduladoras de la vitamina $\mathrm{D}$ puede ayudar a desarrollar nuevas estrategias terapéuticas basadas en el uso de la vitamina $\mathrm{D}$ en el tratamiento de enfermedades inflamatorias.

\section{Contextualizando datos públicos de expresión génica en rutas biológicas}

Como se ha mencionado anteriormente, la primera parte de este proyecto se centró en la creación de una herramienta bioinformática capaz de analizar sets de datos de experimen- 
tos publicados en base de datos abiertas a la comunidad científica. Como describimos en el Capítulo 2, hemos establecido esta herramienta bioinformática capaz de analizar la calidad de estos sets de datos y procesarlos para obtener los parámetros que indican los cambios en la expresión génica los cuales serán contextualizados a nivel de rutas biológicas a través de PathVisio y WikiPathways. Además, la inclusión de otros programas, como Cytoscape, ha permitido incorporar plugins que son capaces de integrar información biológica en nuestro análisis para crear networks biológicas capaces de ilustrar las interacciones moleculares de sistemas biológicos. Cytoscape permite visualizar datos de experimentos moleculares siendo una herramienta de uso sencillo que facilita el entendimiento de las dinámicas biológicas en determinados sistemas en respuesta a la vitamina $\mathrm{D}$.

Una vez establecido el eje PathVisio/WikiPathways/Cytoscape, nuestro objetivo fue testar esta herramienta bioinformática con set de datos de expresión génica de modelos celulares del sistema linfoide que han sido tratados con la vitamina D (Capítulo 3). Esta herramienta fue aplicada a un estudio de vitamina $\mathrm{D}$ que generó un set de datos a través de un estudio de ARN-seq, sin embargo, este estudio no pudo analizar el impacto global de la vitamina $\mathrm{D}$ en el sistema biológico en cuestión. Nuestro objetivo fue aplicar el protocolo PathVisio/ WikiPathways/ Cytoscape a este estudio para proporcionar una nueva perspectiva a este análisis de expresión génica. J A Tamblyn et al [236] exploró el efecto inmuno-modulador de $1,25(\mathrm{OH})_{2} \mathrm{D} 3$ en dos diferentes tipos de células NK (del inglés, natural killer): NK uterinas (del inglés, uNK) procedentes del tejido decidual de la placenta y NK de sangre periférica (del inglés, pNK). El análisis bioinformático, el cual comparó la expresión génica de células uNK con pNK, mostró que, transcripcionalmente hablando, estos tipos celulares son diferentes a pesar de que ambos tipos celulares expresan los mismos marcadores celulares [236]. Esta nueva información apoya la teoría central de que uNK tienen una función completamente diferente en la decidua comparada con la función inmunológica de células pNK en circulación. Este estudio también mostró que el sistema regido por el receptor de la vitamina $\mathrm{D}$ (del inglés, VDR) que responde a $1,25(\mathrm{OH})_{2} \mathrm{D} 3$ fue significativamente más expresado en uNKs en comparación con pNKs, pero comparaciones y análisis posteriores indicaron que $1,25(\mathrm{OH})_{2} \mathrm{D} 3$ no tuvo grandes efectos a nivel de expresión genética entre los dos tipos celulares. La conclusión de esta parte del proyecto fue que la herramienta bioinformática desarrollada inicialmente podía utilizarse para explorar y clarificar set de datos transcriptómicos e identificar mecanismos moleculares específicos para futuros estudios.

Después de demostrar que esta herramienta, con un eje central basado en PathVisio/ WikiPathways/ Cytoscape, puede analizar set de datos de expresión génica, el siguiente paso en nuestro 
proyecto fue aplicar esta herramienta usando set de datos procedentes de diferentes modelos mieloides albergados en repositorios públicos. El principal objetivo de esta parte del proyecto fue realizar un análisis de rutas metabólicas en estos set de datos para proporcionar una nueva perspectiva del papel de 1,25(OH $)_{2} \mathrm{D} 3$ en determinados sistemas biológicos. En concreto nuestro interés se centró en estudiar las acciones de la vitamina D como regulador metabólico del sistema inmune. La comparación se realizó usando una serie de set de datos procedentes de experimentos realizados en modelos celulares mieloides, incluyendo monocitos, células dendríticas y líneas monocíticas celulares, que fueron tratados con $1,25(\mathrm{OH})_{2} \mathrm{D} 3$ usando PathVisio/ WikiPathways/ Cytoscape. Este estudio mostró una clara asociación entre la respuesta del sistema inmune innato a $1,25(\mathrm{OH})_{2} \mathrm{D} 3$ y una reprogramación completa del metabolismo celular. Se observó un claro incremento en la actividad de determinadas rutas metabólicas como glicólisis, cadena de transporte de electrones, fosforilación oxidativa y el ciclo de Krebs. Estos efectos descritos en este estudio son claramente diferentes a los clásicos roles de $1,25(\mathrm{OH})_{2} \mathrm{D} 3$ tales como en rutas anti-proliferativas, las cuales fueron reportadas en nuestro estudio en líneas células monocíticas pero no en modelos celulares primarios relacionados con monocitos y células dendríticas. La identificación de estas nuevas implicaciones por parte de $1,25(\mathrm{OH})_{2} \mathrm{D} 3$ en la reprogramación metabólica celular en diferentes modelos celulares mieloides dió lugar al planteamiento de nuevas hipótesis que fueron la base de la parte final de este proyecto, la cual se basó en el uso de esta plataforma bioinformática para el diseño de nuevos experimentos moleculares.

\section{Explorando el papel de la vitamin D en el inmuno-metabolismo en células dendríticas}

Es bien sabido que la vitamina $\mathrm{D}$ puede regular el desarrollo in vitro de las células dendríticas dando lugar a un fenotipo anti-inflamatorio y tolerogénico. Sin embargo, el mecanismo molecular por el cual $1,25(\mathrm{OH})_{2} \mathrm{D} 3$ logra estos efectos no está totalmente claro. El principal objetivo de la última parte de este proyecto de $\mathrm{PhD}$ fue estudiar este último punto usando los resultados obtenidos en Capítulos 3 y $\mathbf{4}$ para diseñar experimentos moleculares usando nuestro propio modelo celular: células dendríticas procedentes de sangre periférica. El resultado de este trabajo, descrito en el Capítulo 5, se llevó a cabo para investigar la hipótesis de que los efectos teratogénicos de $1,25(\mathrm{OH})_{2} \mathrm{D3}$ se basan en una reprogramación metabólica en células dendríticas. Este estudio se centró en testar esta hipótesis estudiando los efectos directos de $1,25(\mathrm{OH})_{2} \mathrm{D} 3$ en células dendríticas tolerogénicas inmaduras (del inglés, itolDC) que fueron tratadas con $1,25(\mathrm{OH})_{2} \mathrm{D} 3$ en ausencia de estimuladores inmunogénicos. De esta manera, nos centramos en mostrar las respuestas directas a $1,25(\mathrm{OH})_{2} \mathrm{D} 3$ en lugar de la habilidad de $1,25(\mathrm{OH})_{2} \mathrm{D} 3$ de contrarrestar otros moduladores de células dendríticas tales 
como las acciones inmunogénicas de lipopolisacárido (LPS). Los primeros experimentos fueron llevados a cabo para confirmar qué genes involucrados en la cadena de transporte de electrones y ciclo de Krebs eran favorablemente regulados en itolDC comparados con células dendríticas no expuestas a $1,25(\mathrm{OH})_{2} \mathrm{D} 3$. Posteriores estudios funcionales mostraron que existe un incremento en el consumo de oxígeno por parte de itolDC. Este efecto fue observado en células dendríticas por el tratamiento de $1,25(\mathrm{OH})_{2} \mathrm{D} 3$ durante un período largo de tiempo y no en células que fueron expuestas a LPS para generar células dendríticas maduras, ni en células dendríticas expuestas por un periodo corto de tiempo a $1,25(\mathrm{OH})_{2} \mathrm{D} 3$.

Los resultados de los Capítulos 3 y 4 también mostraron que $1,25(\mathrm{OH})_{2} \mathrm{D} 3$ promueve rutas metabólicas centradas en la glucosa. En posteriores estudios moleculares empleamos ${ }^{13} \mathrm{C}_{6}$-glucosa para rastrear el metabolismo de la molécula en itolDC. Estos experimentos se realizaron usando espectrometría de masas para confirmar que, a nivel funcional, la actividad en las rutas metabólicas de glicólisis y el ciclo de Krebs son positivamente reguladas por $1,25(\mathrm{OH})_{2} \mathrm{D} 3$ en itolDC. En estos estudios de rastreo molecular también se empleó ${ }^{13} \mathrm{C}_{5}$-glutamina para determinar si sustratos del ciclo de Krebs pudieran están alterados por $1,25(\mathrm{OH})_{2} \mathrm{D} 3$ en itolDC. A pesar de ello, este último experimento solo demostró que no hubieron apenas cambios metabólicos en el ciclo de Krebs por parte de 1,25(OH) 2 D3, confirmando que el metabolismo de la glucosa es el principal punto de regulación por parte de $1,25(\mathrm{OH})_{2} \mathrm{D} 3$ en células dendríticas. Finalmente, a pesar de que los experimentos de rastreo de metabolitos demostraron una potente respuesta metabólica en células dendríticas tras el tratamiento con $1,25(\mathrm{OH})_{2} \mathrm{D} 3$, estos resultados sólo mostraron datos de homeostasis de metabolitos polares. El análisis de estos mismos experimentos basados en el trazado del consumo ${ }^{13} \mathrm{C}_{6}$-glucosa pero centrados en análisis de metabolitos lipofílicos no-polares, revelaron una significativa incorporación de ${ }^{13} \mathrm{C}$ en palmitato, sugiriendo que $1,25(\mathrm{OH})_{2} \mathrm{D} 3$ promueve un remodelado metabólico que va dirigido a la síntesis de ácidos grasos en itolDC.

De manera colectiva, los resultados de los experimentos moleculares confirmaron los análisis bioinformáticos en modelos celulares mieloides, específicamente en células dendríticas, que la respuesta inmune a $1,25(\mathrm{OH})_{2} \mathrm{D} 3$ se basa en una reprogramación metabólica. Además, los experimentos basados en el trazado de ${ }^{13} \mathrm{C}_{6}$-glucosa indicaron que la glicólisis y el ciclo de Krebs son importantes rutas metabólicas influenciadas por $1,25(\mathrm{OH})_{2} \mathrm{D} 3$. Finalmente, estos mismos experimentos indicaron que $1,25(\mathrm{OH})_{2} \mathrm{D} 3$ es un potente regulador positivo de la síntesis de ácidos grasos en itolDC. Tras estos resultados, propusimos una nueva hipótesis que plantea que la síntesis de ácidos grasos es esencial para promover los efectos tolerogénicos de $1,25(\mathrm{OH})_{2} \mathrm{D3}$ en células dendríticas. Para testar esta hipótesis bloqueamos la ruta 
metabólica de síntesis de ácidos grasos en itolDC para estudiar sus efectos en el fenotipo y función en estas células. Los resultados indicaron que esta inhibición altera la morfología de itolDC y reprime la expresión de CD14 y IL-10 en estas células. Estos resultados sugieren que la capacidad de $1,25(\mathrm{OH})_{2} \mathrm{D} 3$ para inducir el fenotipo tolerogénico en DC es dependiente de una reprogramación metabólica que da lugar a un incremento de síntesis de ácidos grasos.

\section{Conclusión}

Los datos presentados en esta tesis demuestran que un análisis optimizado de set de datos de gran escala y la visualización de estos resultados usando herramientas bioinformáticas puede ayudar a identificar nuevos mecanismos moleculares de la vitamina $\mathrm{D}$ en un modelo celular - en este caso células dendríticas - que previamente ha sido estudiado. Así pues, el empleo de estas herramientas, capaces de hacer un análisis de rutas metabólicas y generación de redes biológicas en set de datos transcriptómicos, ha permitido dar una nueva perspectiva a la función de la vitamina $\mathrm{D}$ en el sistema inmune. Además, con el uso de estas herramientas para analizar set de datos de otros grupos de investigación, hemos podido ayudar a la investigación del papel de la vitamina $\mathrm{D}$ en determinados modelos celulares. Gracias al análisis de rutas metabólicas, hemos generado una serie de resultados que, tras su interpretación, nos ha permitido diseñar nuestros propios experimentos moleculares e investigar con mayor precisión nuestras hipótesis de partida. En este proyecto de doctorado, los experimentos moleculares fueron llevado a cabo en los dos últimos años de su duración. Sin embargo, gracias a que pudimos identificar las rutas metabólicas de interés con mayor eficacia, pudimos optimizar el tiempo de diseño experimental. Este hecho es particularmente importante en el campo de investigación del metabolismo donde técnicas, como la de análisis de trazado de metabolitos, son costosas tanto a nivel económico como en tiempo. Con las dificultades que supone encontrar fondos económicos para la investigación, es importante para el futuro la incorporación de análisis bioinformáticos de set de datos que preceden al trabajo de laboratorio. La conclusión general de este proyecto de doctorado es que la reprogramación metabólica es una interacción crucial entre la vitamina D y el sistema inmune, y es necesario investigar este campo en mayor detalle para mejorar el uso de la vitamina D como terapia en enfermedades inflamatorias autoinmunes. 



\section{Valorization}

\section{$\underline{\text { Science research as a collaborative network }}$}

One of the milestones in bioscience research is the generation of a wide variety of large scale databases that compile very specific information related to molecular biology. Nevertheless, for the correct management of this bulk of data, the science community has established the FAIR basic features of data management which allow their correct usage. First, data must be referenced using identifiers or attributes that are findable in other sources. Secondly, data has to be accessible to everyone in the science community, therefore the platforms and databases containing this information have to be open and a science-based community has to ensure its routinely curation and update. Furthermore, the format of the data has to be interoperable, ensuring that the structure of the data is compatible and suitable with the standard tools of the science community. Finally, the way data and its metadata is stored and written must ease its replication and combination with other features.

The usage of these public repositories that contain datasets and databases from wet-lab experiments should be a crucial part of every research project as we demonstrate in this thesis. Accessible public data from other researchers allows the possibility to exploit it with bioinformatic tools for the purpose to try to explore biology aspects that the authors have not considered in their original hypothesis or to compare/combine it with other data sets to obtain more robust evaluations. Furthermore, a great feature of this bioinformatic pipeline built in this project (Chapter 2), it is possible to process several dataset that share a common pattern (e.g. different cell models with same treatment) allowing their comparison and plotting the results in a friendly way that makes possible a better understanding of how a specific biology system works. Exploiting public data from other researchers and interpreting the results at different molecular biology levels gives a rich and wide insight that is crucial for the first step of a new project: hypothesis establishment. Finally, the re-use and re-analysis of data allows the discovery of new aspects that were not considered in the original approach; as a result its quality and, in consequence, its value increase. 
As mentioned above, generation, storage and analysis of public data are crucial for every project in its first steps; but it is worth mentioning the importance of the actual researcherresearcher interactions from different science fields to have a wider outlook of the system of study. Collaboration between different departments that are specialized in specific niches in science allows the usage of a variety of techniques optimizing the work and time invested in research to validate a hypothesis (Chapter 2). This gap that separates bioinformatics and wet-lab research, is closing bit after bit with the initiative of this kind of collaborative project. Most importantly, young researchers working and being trained in different niches in science widen their perspective, easing their adaptability in further research projects. The joint project that formed the basis for this thesis is a great example of how a good collaboration between departments with different focus and methods at two Universities can result in an interesting, novel and rich study on a very specific aspect of biology and, in consequence, generating data that with high quality and value.

\section{Future perspective: optimizing cell therapy}

The tolerogenic dendritic cell population that we have focused on in this project are crucial for the immune tolerance homeostasis. Disruption in the regulation of the immune tolerance of the individual can lead to anomalies that are known as autoimmune diseases that are characterized by the attack of the host immune system to self components. The immune system has developed mechanisms to avoid these pathologies such as processes to remove immune cells that recognize self-antigens and would provoke an auto-attack. Finally, the immune system also is capable to develop specific cells that inhibit self-reactions processes known as regulatory cells which include the ones focused on this work: tolerogenic DC and T regulatory cells.

The results of our work gave a better understanding of how vitamin D modulates the development of tolerogenic DC based on a metabolic reprogramming. The findings presented in this thesis describe how shifts in metabolism induce changes in the immune function of tolerogenic DC and, in consequence, affect Treg development. This contributes to the notion that immuno-metabolism is an emerging aspect of biochemistry that needs better understanding to explain that key metabolic pathways are essential for the correct execution of immune actions. Decryption of the links between metabolism and immune cell fate can lead to the optimization of cell culture for the generation of specific cell populations that can be used to treat and cure diseases. In that way, this kind of findings can improve personalized cell therapy. 


\section{Promoting multi-disciplined professional researchers}

The bioscience field is living a constant evolution where computation and data management are taking important roles for its progress. These new dry-lab tools have found a niche in bioscience known as bioinformatics but more bridges have to be built to connect this field to the different wet-lab niches, and this thesis is one example of that. As a PhD student, working in these two different science fields has granted me the opportunity to enrich me as a researcher from many different perspectives as well as meeting numerous professionals from both sides. Science progresses in higher levels when its community works as a network where different scientific areas complement each other; optimizing time, work, resources and, in consequence, results.

The community within the University has to keep ensuring the acquisition of multi-disciplined skills to students that will be involved in different fields of science. In computational science, there is a large variety of bioinformatic workflow tools that are crucial for bioscience research that optimize their performance and the quality of the work. That is why knowledge of basic programming languages is important in a professional research career which guarantees the efficiency of work performance in a science project. My personal experience taken from this collaborative project has proven that the integration of skills and knowledge from different fields is an investment that in the long term is very profitable for Universities since it guarantees valid academic professionals that can work in very competitive research projects. 

Acknowledgements 



\section{Acknowledgements}

I could summarize the period of time that took my $\mathrm{PhD}$ in two words: intense and happiness. This joint collaboration has brought me the opportunity to work in two amazing departments from Maastricht University and University of Birmingham; where I met wonderful professionals and friends. Therefore, I have a lot of people to thank.

My first stop was the BiGCaT department of Bioinformatics of Maastricht University. First, Chris thank you for being a great supervisor; your advice and guidance has been crucial to progress in this project in these three years. I always have liked the scientific environment that you have created in the department. Susan, you have been constantly involved in my progress and your supervision has been perfect: your constant feedback and support has been determinant in this work. I will be always grateful for everything you have done. In the first year of the $\mathrm{PhD}$, I had the great opportunity to work with amazing people in the BiGCaT department. Tina, thank you for your advice and feedback in the bioinformatic part of this project. You have been always helpful and kind from the first day that I started working. Lars, thank you for helping me in every technical doubt that I had related to coding and also for the good conversations in the coffee time. Egon, you have been always helpful with your tips and opinions; they always have brought new perspectives to our research questions. Freddie, I always have considered you a great researcher; thank you for your advice, feedback and your delicious cakes! Jonathan, thank you for being a fun and professional colleague; you have helped me with every programming-related issue that I had. Nasim, Denise and Marvin; you came in the second half of my first year but it was enough time to realise that you are amazing and fun colleagues to work with! Thank you. Myrtle, thank you very much for your help in the paper work procedures. During my first stage of my $\mathrm{PhD}$, I had the opportunity to work with researchers from groups linked to our department, such as MaCSBio. Samar and Chaitra, thank you for your work and feedback in many bioinformatic aspects of this project. Michael, thank you for your time and fast replies regarding the bioinformatic aspects; your work and help has been an important part of my project. Finally, the international office: Mirella, Elisa and Ryan. You have been great people to work with and sharing space, moments, trips and good conversations with you has made the whole experience unique. 
Thank you.

Second stop: Institute of Metabolism and System Research (IMSR) of the University of Birmingham. Martin, you have been a perfect supervisor. You have support me during the whole process inside and outside the lab. You have been always available for every one of your students showing that you always have cared about us. Thank you for everything Martin. Ankana, you were the first person that I met when I arrived; giving an amazing tour to the University of Birmingham. From that day to the last, you always have been helpful and kind to me (and to everyone in the lab). For all that, thank you. Danni, thank you for being a good friend inside and outside the laboratory. In my first weeks you helped me a lot showing me the basics of dendritic cell culture and also outside the lab by introducing me to everyone. Carl, I always have thought that you are a great researcher. I will be always grateful for your help and advice during my project. Dean, I think that everyone in the IMSR that has worked or interacted with you can agree that "you have to have a Dean in your lab". From the first day you have been kind and helpful to me. Even when you were busy, you always would find time to assist me with any problems that I had. For all that, thank you Dean. Louisa, from the first day to the last you have been a great scientific reference to this project and to me. Your knowledge in the immune field has been determinant for the development of this project. Thank you so much for your help and kindness.

During the last two years of my $\mathrm{PhD}$ I had the chance to collaborate with amazing researchers in the IMSR department where I could integrate state of the art techniques into my work which grant me the opportunity to learn new aspects of immunometabolism. Dan, thank you for your professional advice regarding metabolism. Your feedback has been determinant for experiment planning and result interpretation. Alpesh, many thanks for your work; your expertise in the mass spectrometry field has made possible to find crucial aspects in my study. Antje, you were one of the first people that helped with my experiments and I will be always grateful for your kindness and professionalism during that first month. Christian and Tom, thank you for your time, support, advice and feedback. Sarah, thank you for your professional advice; I am grateful for your feedback and knowledge in the immune system field. Rowan, you always have found the balance between fun and work. You have been taking care of all students in the science aspects (seminars, workshops, CV course, etc) and social aspects. Thank you for your work! Claire, I met you when you started in the IMSR as a master student and I had to "guide" you through some protocols. From that moment I realised that you were (and will be) a good scientist. Good luck! Cristina, thank you for being a good colleague and friend. Finally, thanks to the football team of Dynamo Keynes 
for organising a weekly football match. It has been fun playing with you guys!

Chloe, you are the person who I spend the most of my $\mathrm{PhD}$ life with. You already know how lucky I feel having lived those experiences with you. Thank you for being the second player in this happy period of my life.

Finally, a few words to my close friends and family. Hernán, a pesar de haber pasado poco tiempo durante nuestras "tosis", tengo la seguridad de que me llevé un gran amigo de Valencia. Siul, a pesar del tiempo y la distancia has sabido estar ahí. Desde pequeños has demostrado ser una gran persona y buen amigo. Verdejo, te tocan algunas palabras por antigüedad. Desde enanos (pasando por la comunión), hemos pasado muchos momentos juntos que, a pesar de los dolores de cabeza que das, me siento afortunado de tenerte como amigo. David, desde el instituto hemos pasado muchas experiencias juntos. Desde el primer momento has sido una de las personas que más me ha influenciado y apoyado. A todos vosotros, gracias por estar ahí.

Por último, a mi familia más cercana. Ana y Pedro, gracias por estar ahí en todo momento; en los fáciles y en los difíciles. Papa, Mama; gracias por la paciencia y el apoyo durante estos años. Es imposible encontrar las palabras para demostraros lo agradecido que estoy. 



\section{References}

[1] Abel, A. M., Yang, C., Thakar, M. S., and Malarkannan, S. (2018). Natural killer cells: development, maturation, and clinical utilization. Frontiers in immunology, 9:1869. Cited on pages 12 and 14.

[2] Abumaree, M., Chamley, L., Badri, M., and El-Muzaini, M. (2012). Trophoblast debris modulates the expression of immune proteins in macrophages: a key to maternal tolerance of the fetal allograft? Journal of reproductive immunology, 94(2):131-141. Cited on page 48.

[3] Adams, J. S. and Hewison, M. (2008). Unexpected actions of vitamin d: new perspectives on the regulation of innate and adaptive immunity. Nature Reviews Endocrinology, 4(2):80. Cited on pages 65, 70, 100, and 117.

[4] Adams, J. S., Ren, S., Liu, P. T., Chun, R. F., Lagishetty, V., Gombart, A. F., Borregaard, N., Modlin, R. L., and Hewison, M. (2009). Vitamin d-directed rheostatic regulation of monocyte antibacterial responses. The Journal of Immunology, 182(7):4289-4295. Cited on pages 95 and 105.

[5] Adorini, L. and Penna, G. (2009). Induction of tolerogenic dendritic cells by vitamin d receptor agonists. In Dendritic cells, pages 251-273. Springer. Cited on page 101.

[6] Afgan, E., Baker, D., Batut, B., Van Den Beek, M., Bouvier, D., Čech, M., Chilton, J., Clements, D., Coraor, N., Grüning, B. A., et al. (2018). The galaxy platform for accessible, reproducible and collaborative biomedical analyses: 2018 update. Nucleic acids research, 46(W1):W537-W544. Cited on page 125.

[7] Agren, R., Liu, L., Shoaie, S., Vongsangnak, W., Nookaew, I., and Nielsen, J. (2013). The raven toolbox and its use for generating a genome-scale metabolic model for penicillium chrysogenum. PLoS computational biology, 9(3):e1002980. Cited on page 30.

[8] Alberts, B. (2018). Molecular biology of the cell. Cited on page 15.

[9] Almerighi, C., Sinistro, A., Cavazza, A., Ciaprini, C., Rocchi, G., and Bergamini, A. (2009). $1 \alpha, 25$-dihydroxyvitamin d3 inhibits cd401-induced pro-inflammatory and immunomodulatory activity in human monocytes. Cytokine, 45(3):190-197. Cited on page 98.

[10] Ambarus, C. A., Noordenbos, T., de Hair, M. J., Tak, P. P., and Baeten, D. L. (2012). Intimal lining layer macrophages but not synovial sublining macrophages display an il-10 polarized-like phenotype in chronic synovitis. Arthritis research \& therapy, 14(2):R74. Cited on page 11.

[11] Anderson, A. E., Swan, D., Wong, O., Buck, M., Eltherington, O., Harry, R., Patterson, A., Pratt, A., Reynolds, G., Doran, J.-P., et al. (2017). Tolerogenic dendritic cells generated with dexamethasone and vitamin $\mathrm{d} 3$ regulate rheumatoid arthritis cd4+ $\mathrm{t}$ cells partly via transforming growth factor- $\beta 1$. Clinical \& Experimental Immunology, 187(1):113-123. Cited on page 126 . 
[12] Athar, A., Füllgrabe, A., George, N., Iqbal, H., Huerta, L., Ali, A., Snow, C., Fonseca, N. A., Petryszak, R., Papatheodorou, I., et al. (2019). Arrayexpress update-from bulk to single-cell expression data. Nucleic acids research, 47(D1):D711-D715. Cited on page 20.

[13] Atri, C., Guerfali, F. Z., and Laouini, D. (2018). Role of human macrophage polarization in inflammation during infectious diseases. International journal of molecular sciences, 19(6):1801. Cited on page 11.

[14] Bacchetta, J., Chun, R. F., Gales, B., Zaritsky, J. J., Leroy, S., Wesseling-Perry, K., Boregaard, N., Rastogi, A., Salusky, I. B., and Hewison, M. (2014a). Antibacterial responses by peritoneal macrophages are enhanced following vitamin $\mathrm{d}$ supplementation. PloS one, 9(12):e116530. Cited on pages 95 and 125.

[15] Bacchetta, J., Zaritsky, J. J., Sea, J. L., Chun, R. F., Lisse, T. S., Zavala, K., Nayak, A., Wesseling-Perry, K., Westerman, M., Hollis, B. W., et al. (2014b). Suppression of iron-regulatory hepcidin by vitamin d. Journal of the American Society of Nephrology, 25(3):564-572. Cited on pages 101 and 125.

[16] Barrett, T. and Edgar, R. (2006). Mining microarray data at ncbi's gene expression omnibus (geo). In Gene Mapping, Discovery, and Expression, pages 175-190. Springer. Cited on page 74 .

[17] Bendix, M., Dige, A., Deleuran, B., Dahlerup, J., Jørgensen, S. P., Bartels, L., Husted, L., Harsløf, T., Langdahl, B., and Agnholt, J. (2015). Flow cytometry detection of vitamin $\mathrm{d}$ receptor changes during vitamin d treatment in crohn's disease. Clinical \& Experimental Immunology, 181(1):19-28. Cited on page 50.

[18] Berer, A., Stöckl, J., Majdic, O., Wagner, T., Kollars, M., Lechner, K., Geissler, K., and Oehler, L. (2000). 1, 25-dihydroxyvitamin d3 inhibits dendritic cell differentiation and maturation in vitro. Experimental hematology, 28(5):575-583. Cited on page 116.

[19] Bertani, F. R., Mozetic, P., Fioramonti, M., Iuliani, M., Ribelli, G., Pantano, F., Santini, D., Tonini, G., Trombetta, M., Businaro, L., et al. (2017). Classification of $\mathrm{m} 1 / \mathrm{m} 2$ polarized human macrophages by label-free hyperspectral reflectance confocal microscopy and multivariate analysis. Scientific reports, 7(1):1-9. Cited on page 10.

[20] BHALLA, A. K., AMENTO, E. P., CLEMENS, T. L., HOLICK, M. F., and KRANE, S. M. (1983). Specific high-affinity receptors for 1, 25-dihydroxyvitamin d3 in human peripheral blood mononuclear cells: presence in monocytes and induction in tymphocytes following activation. The Journal of Clinical Endocrinology \& Metabolism, 57(6):13081310. Cited on pages 15, 71, and 100 .

[21] Bhalla, A. K., Amento, E. P., Serog, B., and Glimcher, L. H. (1984). 1, $25-$ dihydroxyvitamin $\mathrm{d} 3$ inhibits antigen-induced t cell activation. The Journal of Immunology, 133(4):1748-1754. Cited on page 17.

[22] Bindea, G., Mlecnik, B., Hackl, H., Charoentong, P., Tosolini, M., Kirilovsky, A., Fridman, W.-H., Pagès, F., Trajanoski, Z., and Galon, J. (2009). Cluego: a cytoscape plug-in to decipher functionally grouped gene ontology and pathway annotation networks. Bioinformatics, 25(8):1091-1093. Cited on pages 30 and 72.

[23] Bischoff-Ferrari, H. A., Dawson-Hughes, B., Willett, W. C., Staehelin, H. B., Bazemore, M. G., Zee, R. Y., and Wong, J. B. (2004). Effect of vitamin d on falls: a meta-analysis. Jama, 291(16):1999-2006. Cited on page 127.

[24] Bohler, A., Eijssen, L. M., van Iersel, M. P., Leemans, C., Willighagen, E. L., Kutmon, M., Jaillard, M., and Evelo, C. T. (2015). Automatically visualise and analyse data on pathways using pathvisiorpc from any programming environment. Bmc Bioinformatics, 16(1):267. Cited on page 124. 
[25] Bohler, A., Wu, G., Kutmon, M., Pradhana, L. A., Coort, S. L., Hanspers, K., Haw, R., Pico, A. R., and Evelo, C. T. (2016). Reactome from a wikipathways perspective. PLoS computational biology, 12(5). Cited on page 52.

[26] Boonstra, A., Barrat, F. J., Crain, C., Heath, V. L., Savelkoul, H. F., and O'Garra, A. (2001). $1 \alpha, 25$-dihydroxyvitamin $\mathrm{d} 3$ has a direct effect on naive cd4+ $\mathrm{t}$ cells to enhance the development of th2 cells. The Journal of Immunology, 167(9):4974-4980. Cited on page 17.

[27] Bossé, Y., Maghni, K., and Hudson, T. J. (2007). 1 $\alpha$, 25-dihydroxy-vitamin d3 stimulation of bronchial smooth muscle cells induces autocrine, contractility, and remodeling processes. Physiological genomics, 29(2):161-168. Cited on page 29.

[28] Brennan, A., Katz, D. R., Nunn, J., Barker, S., Hewison, M., Fraher, L. J., and O'Riordan, J. L. (1987). Dendritic cells from human tissues express receptors for the immunoregulatory vitamin d3 metabolite, dihydroxycholecalciferol. Immunology, 61(4):457. Cited on page 15 .

[29] Brinkmann, V. and Zychlinsky, A. (2012). Neutrophil extracellular traps: is immunity the second function of chromatin? Journal of cell biology, 198(5):773-783. Cited on page 9.

[30] Brosbøl-Ravnborg, A., Bundgaard, B., and Höllsberg, P. (2013). Synergy between vitamin $\mathrm{d} 3$ and toll-like receptor agonists regulates human dendritic cell response during maturation. Clinical and Developmental Immunology, 2013. Cited on page 101.

[31] Bulmer, J. N., Williams, P. J., and Lash, G. E. (2009). Immune cells in the placental bed. International Journal of Developmental Biology, 54(2-3):281-294. Cited on pages 14 and 48.

[32] Calton, E. K., Keane, K. N., Soares, M. J., Rowlands, J., and Newsholme, P. (2016). Prevailing vitamin $\mathrm{d}$ status influences mitochondrial and glycolytic bioenergetics in peripheral blood mononuclear cells obtained from adults. Redox biology, 10:243-250. Cited on page 96.

[33] Campbell, G. R. and Spector, S. A. (2012). Autophagy induction by vitamin d inhibits both mycobacterium tuberculosis and human immunodeficiency virus type 1. Autophagy, 8(10):1523-1525. Cited on page 17.

[34] CANTLEY, L. K., RUSSELL, J., LETTIERI, D., and SHERWOOD, L. M. (1985). 1, 25dihydroxyvitamin $\mathrm{d} 3$ suppresses parathyroid hormone secretion from bovine parathyroid cells in tissue culture. Endocrinology, 117(5):2114-2119. Cited on page 7.

[35] Cantorna, M. T., Snyder, L., Lin, Y.-D., and Yang, L. (2015). Vitamin d and 1, 25 (oh) $2 \mathrm{~d}$ regulation of t cells. Nutrients, 7(4):3011-3021. Cited on page 17.

[36] Carlberg, C. (2003). Current understanding of the function of the nuclear vitamin $d$ receptor in response to its natural and synthetic ligands. In Vitamin D Analogs in Cancer Prevention and Therapy, pages 29-42. Springer. Cited on page 7.

[37] Carlberg, C. (2019). Nutrigenomics of vitamin d. Nutrients, 11(3):676. Cited on page 68.

[38] Carroll, R. G., Zasłona, Z., Galván-Peña, S., Koppe, E. L., Sévin, D. C., Angiari, S., Triantafilou, M., Triantafilou, K., Modis, L. K., and O’Neill, L. A. (2018). An unexpected link between fatty acid synthase and cholesterol synthesis in proinflammatory macrophage activation. Journal of Biological Chemistry, 293(15):5509-5521. Cited on page 118.

[39] Chan, K. L., Pillon, N. J., Sivaloganathan, D. M., Costford, S. R., Liu, Z., Théret, M., Chazaud, B., and Klip, A. (2015). Palmitoleate reverses high fat-induced proinflammatory macrophage polarization via amp-activated protein kinase (ampk). Journal of Biological Chemistry, 290(27):16979-16988. Cited on page 118. 
[40] Chaudhry, A., Samstein, R. M., Treuting, P., Liang, Y., Pils, M. C., Heinrich, J.-M., Jack, R. S., Wunderlich, F. T., Brüning, J. C., Müller, W., et al. (2011). Interleukin-10 signaling in regulatory $\mathrm{t}$ cells is required for suppression of th17 cell-mediated inflammation. Immunity, 34(4):566-578. Cited on pages 12 and 14.

[41] Chen, S., Sims, G. P., Chen, X. X., Gu, Y. Y., Chen, S., and Lipsky, P. E. (2007). Modulatory effects of 1,25-dihydroxyvitamin $\mathrm{d} 3$ on human $\mathrm{b}$ cell differentiation. The Journal of Immunology, 179(3):1634-1647. Cited on page 17.

[42] Chinen, T., Kannan, A. K., Levine, A. G., Fan, X., Klein, U., Zheng, Y., Gasteiger, G., Feng, Y., Fontenot, J. D., and Rudensky, A. Y. (2016). An essential role for the il-2 receptor in t reg cell function. Nature immunology, 17(11):1322-1333. Cited on page 14.

[43] Chiossone, L., Dumas, P.-Y., Vienne, M., and Vivier, E. (2018). Natural killer cells and other innate lymphoid cells in cancer. Nature Reviews Immunology, 18(11):671-688. Cited on page 66.

[44] Chou, C.-H., Shrestha, S., Yang, C.-D., Chang, N.-W., Lin, Y.-L., Liao, K.-W., Huang, W.-C., Sun, T.-H., Tu, S.-J., Lee, W.-H., et al. (2017). mirtarbase update 2018: a resource for experimentally validated microrna-target interactions. Nucleic acids research, 46(D1):D296-D302. Cited on pages 19 and 38.

[45] Christakos, S., Dhawan, P., Porta, A., Mady, L. J., and Seth, T. (2011). Vitamin d and intestinal calcium absorption. Molecular and cellular endocrinology, 347(1-2):25-29. Cited on page 8 .

[46] Chun, R. F., Liu, N. Q., Lee, T., Schall, J. I., Denburg, M. R., Rutstein, R. M., Adams, J. S., Zemel, B. S., Stallings, V. A., and Hewison, M. (2015). Vitamin d supplementation and antibacterial immune responses in adolescents and young adults with hiv/aids. The Journal of steroid biochemistry and molecular biology, 148:290-297. Cited on page 95.

[47] Chun, R. F., Liu, P. T., Modlin, R. L., Adams, J. S., and Hewison, M. (2014). Impact of vitamin d on immune function: lessons learned from genome-wide analysis. Frontiers in physiology, 5:151. Cited on pages 18, 68, 71, 95, 98, and 100.

[48] Çimen, I., Kocatürk, B., Koyuncu, S., Tufanlı, Ö., Onat, U. I., Yıldırım, A. D., Apaydın, O., Demirsoy, Ş., Aykut, Z. G., Nguyen, U. T., et al. (2016). Prevention of atherosclerosis by bioactive palmitoleate through suppression of organelle stress and inflammasome activation. Science Translational Medicine, 8(358):358ra126-358ra126. Cited on page 118.

[49] Clausen, J., Vergeiner, B., Enk, M., Petzer, A. L., Gastl, G., and Gunsilius, E. (2003). Functional significance of the activation-associated receptors cd25 and cd69 on human nk-cells and nk-like t-cells. Immunobiology, 207(2):85-93. Cited on page 50.

[50] Clough, E. and Barrett, T. (2016). The gene expression omnibus database. In Statistical Genomics, pages 93-110. Springer. Cited on page 29.

[51] Collin, M., McGovern, N., and Haniffa, M. (2013). Human dendritic cell subsets. Immunology, 140(1):22-30. Cited on page 11.

[52] Consortium, E. P. et al. (2007). Birney e, stamatoyannopoulos ja, dutta a, guigo r. Technical report, Gingeras TR, Margulies EH, Weng Z, Snyder M, Dermitzakis ET, et al. 2007 ... Cited on page 95.

[53] de Souza, C. O., Vannice, G. K., Rosa Neto, J. C., and Calder, P. C. (2018). Is palmitoleic acid a plausible nonpharmacological strategy to prevent or control chronic metabolic and inflammatory disorders? Molecular nutrition \& food research, 62(1):1700504. Cited on page 127.

[54] Deeb, K. K., Trump, D. L., and Johnson, C. S. (2007). Vitamin d signalling pathways in cancer: potential for anticancer therapeutics. Nature reviews cancer, 7(9):684-700. Cited on pages 6 and 8 . 
[55] Del Valle, H. B., Yaktine, A. L., Taylor, C. L., Ross, A. C., et al. (2011). Dietary reference intakes for calcium and vitamin D. National Academies Press. Cited on page 4.

[56] DeLuca, H. F. (2008). Evolution of our understanding of vitamin d. Nutrition reviews, 66(suppl_2):S73-S87. Cited on page 16.

[57] DeLuca, H. F. (2014). History of the discovery of vitamin d and its active metabolites. BoneKEy reports, 3. Cited on page 2.

[58] Di Nisio, A., De Toni, L., Sabovic, I., Rocca, M. S., De Filippis, V., Opocher, G., Azzena, B., Vettor, R., Plebani, M., and Foresta, C. (2017). Impaired release of vitamin d in dysfunctional adipose tissue: new cues on vitamin d supplementation in obesity. The Journal of Clinical Endocrinology \& Metabolism, 102(7):2564-2574. Cited on page 8.

[59] Dobin, A., Davis, C. A., Schlesinger, F., Drenkow, J., Zaleski, C., Jha, S., Batut, P., Chaisson, M., and Gingeras, T. R. (2013). Star: ultrafast universal rna-seq aligner. Bioinformatics, 29(1):15-21. Cited on page 52.

[60] Domogalla, M. P., Rostan, P. V., Raker, V. K., and Steinbrink, K. (2017). Tolerance through education: how tolerogenic dendritic cells shape immunity. Frontiers in immunology, 8:1764. Cited on pages 12 and 118.

[61] Doncheva, N. T., Morris, J. H., Gorodkin, J., and Jensen, L. J. (2018). Cytoscape stringapp: network analysis and visualization of proteomics data. Journal of proteome research, 18(2):623-632. Cited on page 24.

[62] Doroudi, M., Schwartz, Z., and Boyan, B. D. (2015). Membrane-mediated actions of 1, 25-dihydroxy vitamin $\mathrm{d} 3$ : A review of the roles of phospholipase a 2 activating protein and ca2+/calmodulin-dependent protein kinase ii. The Journal of steroid biochemistry and molecular biology, 147:81-84. Cited on page 7.

[63] Edgar, R., Domrachev, M., and Lash, A. E. (2002). Gene expression omnibus: Ncbi gene expression and hybridization array data repository. Nucleic acids research, 30(1):207210. Cited on pages 20 and 32.

[64] Eijssen, L. M., Jaillard, M., Adriaens, M. E., Gaj, S., de Groot, P. J., Müller, M., and Evelo, C. T. (2013). User-friendly solutions for microarray quality control and preprocessing on arrayanalysis. org. Nucleic acids research, 41(W1):W71-W76. Cited on pages 30,33 , and 74 .

[65] Epelman, S., Lavine, K. J., and Randolph, G. J. (2014). Origin and functions of tissue macrophages. Immunity, 41(1):21-35. Cited on page 10.

[66] Erlebacher, A. (2013). Immunology of the maternal-fetal interface. Annual review of immunology, 31:387-411. Cited on page 48.

[67] Evans, K. N., Bulmer, J. N., Kilby, M. D., and Hewison, M. (2004). Vitamin d and placental-decidual function. Journal of the Society for Gynecologic Investigation, 11(5):263-271. Cited on page 49.

[68] Fabregat, A., Jupe, S., Matthews, L., Sidiropoulos, K., Gillespie, M., Garapati, P., Haw, R., Jassal, B., Korninger, F., May, B., et al. (2017). The reactome pathway knowledgebase. Nucleic acids research, 46(D1):D649-D655. Cited on page 37.

[69] Feldman, D., Pike, J., Bouillon, R., Giovannucci, E., Goltzman, D., and Hewison, M. (2017). Vitamin D: Volume 1: Biochemistry, Physiology and Diagnostics. Elsevier Science. Cited on pages 2, 4, 5, 7, and 127.

[70] Felicidade, I., Sartori, D., Coort, S. L., Semprebon, S. C., Niwa, A. M., D’Epiro, G. F. R., Biazi, B. I., Marques, L. A., Evelo, C. T., Mantovani, M. S., et al. (2018). Role of $1 \alpha, 25$-dihydroxyvitamin $\mathrm{d} 3$ in adipogenesis of sgbs cells: New insights into human preadipocyte proliferation. Cellular Physiology and Biochemistry, 48(1):397-408. Cited on page 29 . 
[71] Ferlazzo, G. and Carrega, P. (2012). Natural killer cell distribution and trafficking in human tissues. Frontiers in immunology, 3:347. Cited on page 14.

[72] Ferreira, G. B., Vanherwegen, A.-S., Eelen, G., Gutiérrez, A. C. F., Van Lommel, L., Marchal, K., Verlinden, L., Verstuyf, A., Nogueira, T., Georgiadou, M., et al. (2015). Vitamin $\mathrm{d} 3$ induces tolerance in human dendritic cells by activation of intracellular metabolic pathways. Cell reports, 10(5):711-725. Cited on pages xi, 34, 36, 40, 41, 43, 44, 71, 74, 95, 96, 98, 101, 116, 117, and 126.

[73] Flavin, R., Peluso, S., Nguyen, P. L., and Loda, M. (2010). Fatty acid synthase as a potential therapeutic target in cancer. Future oncology, 6(4):551-562. Cited on page 115.

[74] Flórez-Grau, G., Zubizarreta, I., Cabezón, R., Villoslada, P., and Benitez-Ribas, D. (2018). Tolerogenic dendritic cells as a promising antigen-specific therapy in the treatment of multiple sclerosis and neuromyelitis optica from preclinical to clinical trials. Frontiers in immunology, 9:1169. Cited on page 126.

[75] Fraser, D. and Kodicek, E. (1973). Regulation of 25-hydroxycholecalciferol1-hydroxylase activity in kidney by parathyroid hormone. Nature New Biology, 241(110):163-166. Cited on page 7.

[76] Freemerman, A. J., Johnson, A. R., Sacks, G. N., Milner, J. J., Kirk, E. L., Troester, M. A., Macintyre, A. N., Goraksha-Hicks, P., Rathmell, J. C., and Makowski, L. (2014). Metabolic reprogramming of macrophages glucose transporter 1 (glut1)-mediated glucose metabolism drives a proinflammatory phenotype. Journal of Biological Chemistry, 289(11):7884-7896. Cited on page 125.

[77] Frolova, A. I. and Moley, K. H. (2011). Quantitative analysis of glucose transporter mrnas in endometrial stromal cells reveals critical role of glut1 in uterine receptivity. Endocrinology, 152(5):2123-2128. Cited on page 66.

[78] Fukui, A., Kamoi, M., Funamizu, A., Fuchinoue, K., Chiba, H., Yokota, M., Fukuhara, R., and Mizunuma, H. (2015). Nk cell abnormality and its treatment in women with reproductive failures such as recurrent pregnancy loss, implantation failures, preeclampsia, and pelvic endometriosis. Reproductive medicine and biology, 14(4):151-157. Cited on page 18 .

[79] Gambhir, V., Kim, J., Siddiqui, S., Taylor, M., Byford, V., Petrof, E. O., Jones, G., and Basta, S. (2011). Influence of 1, 25-dihydroxy vitamin d3 on tlr4-induced activation of antigen presenting cells is dependent on the order of receptor engagement. Immunobiology, 216(9):988-996. Cited on page 101.

[80] Gamliel, M., Goldman-Wohl, D., Isaacson, B., Gur, C., Stein, N., Yamin, R., Berger, M., Grunewald, M., Keshet, E., Rais, Y., et al. (2018). Trained memory of human uterine nk cells enhances their function in subsequent pregnancies. Immunity, 48(5):951-962. Cited on page 68.

[81] Garcia, A. M., Kutmon, M., Eijssen, L., Hewison, M., Evelo, C. T., and Coort, S. L. (2018). Pathway analysis of transcriptomic data shows immunometabolic effects of vitamin d. Journal of molecular endocrinology, 60(2):95-108. Cited on pages 8, 30, 34, $39,52,67,101,116$, and 125.

[82] Gardner, A. and Ruffell, B. (2016). Dendritic cells and cancer immunity. Trends in immunology, 37(12):855-865. Cited on page 11.

[83] Garten, A., Grohmann, T., Kluckova, K., Lavery, G. G., Kiess, W., and Penke, M. (2019). Sorafenib-induced apoptosis in hepatocellular carcinoma is reversed by sirt1. International journal of molecular sciences, 20(16):4048. Cited on page 105.

[84] Garzia, E., Clauser, R., Persani, L., Borgato, S., Bulfamante, G., Avagliano, L., Quadrelli, F., and Marconi, A. M. (2013). Prolactin and proinflammatory cytokine 
expression at the fetomaternal interface in first trimester miscarriage. Fertility and sterility, 100(1):108-115. Cited on page 65.

[85] Georgiev, H., Ravens, I., Papadogianni, G., and Bernhardt, G. (2018). Coming of age: Cd96 emerges as modulator of immune responses. Frontiers in immunology, 9:1072. Cited on page 53.

[86] Gibbs, D. (1994). Rickets and the crippled child: an historical perspective. Journal of the Royal Society of Medicine, 87(12):729. Cited on page 3.

[87] Glisson, F., Bate, G., Regemorter, A., Sandler, L., and Beaumont, R. (1650). De rachitide sive Morbo puerili, qui vulgò The Rickets dicitur, tractatus. typis Guil. Du-gardi : impensis Laurentii Sadler, \& Roberti Beaumont. Cited on page 3.

[88] Gray, T. K., Lester, G. E., and Lorenc, R. S. (1979). Evidence for extra-renal 1 alphahydroxylation of 25-hydroxyvitamin d3 in pregnancy. Science, 204(4399):1311-1313. Cited on page 49.

[89] Gregori, S., Tomasoni, D., Pacciani, V., Scirpoli, M., Battaglia, M., Magnani, C. F., Hauben, E., and Roncarolo, M.-G. (2010). Differentiation of type $1 \mathrm{t}$ regulatory cells $(\operatorname{tr} 1)$ by tolerogenic dc-10 requires the il-10-dependent ilt4/hla-g pathway. Blood, The Journal of the American Society of Hematology, 116(6):935-944. Cited on page 17.

[90] Guak, H., Al Habyan, S., Ma, E. H., Aldossary, H., Al-Masri, M., Won, S. Y., Ying, T., Fixman, E. D., Jones, R. G., McCaffrey, L. M., et al. (2018). Glycolytic metabolism is essential for ccr7 oligomerization and dendritic cell migration. Nature communications, 9(1):1-12. Cited on page 125.

[91] Gunasekar, P., Swier, V. J., Fleegel, J. P., Boosani, C. S., Radwan, M. M., and Agrawal, D. K. (2018). Vitamin d and macrophage polarization in epicardial adipose tissue of atherosclerotic swine. PloS one, 13(10). Cited on page 17.

[92] Gunton, J. E. and Girgis, C. M. (2018). Vitamin d and muscle. Bone reports, 8:163-167. Cited on page 8.

[93] Hanna, J., Goldman-Wohl, D., Hamani, Y., Avraham, I., Greenfield, C., NatansonYaron, S., Prus, D., Cohen-Daniel, L., Arnon, T. I., Manaster, I., et al. (2006). Decidual nk cells regulate key developmental processes at the human fetal-maternal interface. Nature medicine, 12(9):1065-1074. Cited on page 65.

[94] Hanna, J., Wald, O., Goldman-Wohl, D., Prus, D., Markel, G., Gazit, R., Katz, G., Haimov-Kochman, R., Fujii, N., Yagel, S., et al. (2003). Cxcl12 expression by invasive trophoblasts induces the specific migration of cd16-human natural killer cells. Blood, 102(5):1569-1577. Cited on page 67.

[95] Haussler, M. R., Jurutka, P. W., Mizwicki, M., and Norman, A. W. (2011). Vitamin d receptor (vdr)-mediated actions of $1 \alpha, 25$ (oh) 2vitamin d3: genomic and non-genomic mechanisms. Best practice \& research Clinical endocrinology \& metabolism, 25(4):543559. Cited on page 7.

[96] Haussler, M. R., Whitfield, G. K., Kaneko, I., Haussler, C. A., Hsieh, D., Hsieh, J.-C., and Jurutka, P. W. (2013). Molecular mechanisms of vitamin d action. Calcified tissue international, 92(2):77-98. Cited on page 100.

[97] Hewison, M. (2010). Antibacterial effects of vitamin d. nature reviews endocrinology 7 337-345. Cited on pages 70, 71, and 117.

[98] Hewison, M. (2011). Antibacterial effects of vitamin d. Nature Reviews Endocrinology, 7(6):337. Cited on pages 16, 100, and 125.

[99] Hewison, M. (2012). An update on vitamin d and human immunity. Clinical endocrinology, 76(3):315-325. Cited on pages 100 and 117. 
[100] Hewison, M., Dabrowski, M., Vadher, S., Faulkner, L., Cockerill, F. J., Brickell, P. M., O'Riordan, J., and Katz, D. R. (1996). Antisense inhibition of vitamin d receptor expression induces apoptosis in monoblastoid u937 cells. The Journal of Immunology, 156(11):4391-4400. Cited on page 98.

[101] Hewison, M., Freeman, L., Hughes, S. V., Evans, K. N., Bland, R., Eliopoulos, A. G., Kilby, M. D., Moss, P. A., and Chakraverty, R. (2003). Differential regulation of vitamin $\mathrm{d}$ receptor and its ligand in human monocyte-derived dendritic cells. The Journal of Immunology, 170(11):5382-5390. Cited on pages 71, 100, 101, and 116.

[102] Hii, C. S. and Ferrante, A. (2016). The non-genomic actions of vitamin d. Nutrients, 8(3):135. Cited on pages 8, 40, and 68.

[103] Hoffman, W., Lakkis, F. G., and Chalasani, G. (2016). B cells, antibodies, and more. Clinical Journal of the American Society of Nephrology, 11(1):137-154. Cited on page 15.

[104] Holick, M. F., Binkley, N. C., Bischoff-Ferrari, H. A., Gordon, C. M., Hanley, D. A., Heaney, R. P., Murad, M. H., and Weaver, C. M. (2011). Evaluation, treatment, and prevention of vitamin d deficiency: an endocrine society clinical practice guideline. The Journal of Clinical Endocrinology \& Metabolism, 96(7):1911-1930. Cited on pages 4 and 127.

[105] Hopkins, F. G. (1912). Feeding experiments illustrating the importance of accessory factors in normal dietaries. The Journal of physiology, 44(5-6):425-460. Cited on page 2.

[106] Høyer-Hansen, M., Bastholm, L., Mathiasen, I., Elling, F., and Jäättelä, M. (2005). Vitamin d analog eb1089 triggers dramatic lysosomal changes and beclin 1-mediated autophagic cell death. Cell Death \& Differentiation, 12(10):1297-1309. Cited on page 125.

[107] Ibrahim, J., Nguyen, A. H., Rehman, A., Ochi, A., Jamal, M., Graffeo, C. S., Henning, J. R., Zambirinis, C. P., Fallon, N. C., Barilla, R., et al. (2012). Dendritic cell populations with different concentrations of lipid regulate tolerance and immunity in mouse and human liver. Gastroenterology, 143(4):1061-1072. Cited on page 118.

[108] Iqbal, S. and Kumar, A. (2015). Characterization of in vitro generated human polarized macrophages. J Clin Cell Immunol, 6(380.10):4172. Cited on page 11.

[109] Jaguin, M., Houlbert, N., Fardel, O., and Lecureur, V. (2013). Polarization profiles of human m-csf-generated macrophages and comparison of m1-markers in classically activated macrophages from gm-csf and m-csf origin. Cellular immunology, 281(1):51-61. Cited on page 11 .

[110] Jeffery, L. E., Burke, F., Mura, M., Zheng, Y., Qureshi, O. S., Hewison, M., Walker, L. S., Lammas, D. A., Raza, K., and Sansom, D. M. (2009). 1, 25-dihydroxyvitamin $\mathrm{d} 3$ and il-2 combine to inhibit t cell production of inflammatory cytokines and promote development of regulatory t cells expressing ctla-4 and foxp3. The Journal of Immunology, 183(9):5458-5467. Cited on pages 17, 100, and 125.

[111] Jeffery, L. E., Henley, P., Marium, N., Filer, A., Sansom, D. M., Hewison, M., and Raza, K. (2018). Decreased sensitivity to 1, 25-dihydroxyvitamin d3 in t cells from the rheumatoid joint. Journal of autoimmunity, 88:50-60. Cited on page 125.

[112] Jeffery, L. E., Raza, K., and Hewison, M. (2016). Vitamin d in rheumatoid arthritis-towards clinical application. Nature Reviews Rheumatology, 12(4):201. Cited on pages 70 and 71 .

[113] Jeffery, L. E., Wood, A. M., Qureshi, O. S., Hou, T. Z., Gardner, D., Briggs, Z., Kaur, S., Raza, K., and Sansom, D. M. (2012). Availability of 25-hydroxyvitamin d3 to apcs controls the balance between regulatory and inflammatory t cell responses. The Journal of Immunology, 189(11):5155-5164. Cited on pages 51, 71, and 126. 
[114] Jiang, X., O’Reilly, P. F., Aschard, H., Hsu, Y.-H., Richards, J. B., Dupuis, J., Ingelsson, E., Karasik, D., Pilz, S., Berry, D., et al. (2018). Genome-wide association study in 79,366 european-ancestry individuals informs the genetic architecture of 25-hydroxyvitamin $\mathrm{d}$ levels. Nature communications, 9(1):1-12. Cited on page 128.

[115] Jokhi, P. P., King, A., Sharkey, A. M., Smith, S. K., and Loke, Y. W. (1994). Screening for cytokine messenger ribonucleic acids in purified human decidual lymphocyte populations by the reverse-transcriptase polymerase chain reaction. The Journal of Immunology, 153(10):4427-4435. Cited on page 65.

[116] Joshi, S., Pantalena, L.-C., Liu, X. K., Gaffen, S. L., Liu, H., Rohowsky-Kochan, C., Ichiyama, K., Yoshimura, A., Steinman, L., Christakos, S., et al. (2011). 1, 25dihydroxyvitamin $\mathrm{d} 3$ ameliorates th17 autoimmunity via transcriptional modulation of interleukin-17a. Molecular and cellular biology, 31(17):3653-3669. Cited on page 17.

[117] Kato, S. (2000). The function of vitamin d receptor in vitamin d action. The Journal of Biochemistry, 127(5):717-722. Cited on page 7.

[118] Keskin, D. B., Allan, D. S., Rybalov, B., Andzelm, M. M., Stern, J. N., Kopcow, H. D., Koopman, L. A., and Strominger, J. L. (2007). $\operatorname{Tgf} \beta$ promotes conversion of cd16+ peripheral blood nk cells into cd16- nk cells with similarities to decidual nk cells. Proceedings of the National Academy of Sciences, 104(9):3378-3383. Cited on page 67.

[119] Khaw, K.-T., Stewart, A. W., Waayer, D., Lawes, C. M., Toop, L., Camargo Jr, C. A., and Scragg, R. (2017). Effect of monthly high-dose vitamin d supplementation on falls and non-vertebral fractures: secondary and post-hoc outcomes from the randomised, doubleblind, placebo-controlled vida trial. The lancet Diabetes \& endocrinology, 5(6):438-447. Cited on pages 126 and 127.

[120] King, A., Balendran, N., Wooding, P., Carter, N., and Loke, Y. (1991). Cd3-leukocytes present in the human uterus during early placentation: Phenotypic and morphologic characterization of the cd56. Journal of Immunology Research, 1(3):169-190. Cited on page 14.

[121] Kolesnikov, N., Hastings, E., Keays, M., Melnichuk, O., Tang, Y. A., Williams, E., Dylag, M., Kurbatova, N., Brandizi, M., Burdett, T., et al. (2014). Arrayexpress update-simplifying data submissions. Nucleic acids research, 43(D1):D1113-D1116. Cited on pages 32 and 74 .

[122] Kommagani, R., Szwarc, M. M., Kovanci, E., Gibbons, W. E., Putluri, N., Maity, S., Creighton, C. J., Sreekumar, A., DeMayo, F. J., Lydon, J. P., et al. (2013). Acceleration of the glycolytic flux by steroid receptor coactivator- 2 is essential for endometrial decidualization. PLoS genetics, 9(10). Cited on page 66.

[123] Koopman, L. A., Kopcow, H. D., Rybalov, B., Boyson, J. E., Orange, J. S., Schatz, F., Masch, R., Lockwood, C. J., Schachter, A. D., Park, P. J., et al. (2003). Human decidual natural killer cells are a unique nk cell subset with immunomodulatory potential. The Journal of experimental medicine, 198(8):1201-1212. Cited on pages 49, 53, 65, and 66.

[124] Kopcow, H. D., Allan, D. S., Chen, X., Rybalov, B., Andzelm, M. M., Ge, B., and Strominger, J. L. (2005). Human decidual nk cells form immature activating synapses and are not cytotoxic. Proceedings of the National Academy of Sciences, 102(43):1556315568. Cited on page 66.

[125] Kovacs, C. S. (2005). Calcium and bone metabolism during pregnancy and lactation. Journal of mammary gland biology and neoplasia, 10(2):105-118. Cited on page 4.

[126] Kovacs, E., Meichsner-Frauli, M., and Ludwig, H. (1992). Interleukin-2 receptor positive cells in human decidua during the first trimester of pregnancy and their association with macrophages. Archives of gynecology and obstetrics, 251(2):93-100. Cited on 
page 65 .

[127] Kozomara, A., Birgaoanu, M., and Griffiths-Jones, S. (2019). mirbase: from microrna sequences to function. Nucleic acids research, 47(D1):D155-D162. Cited on page 19.

[128] Kreutz, M., Andreesen, R., Krause, S. W., Szabo, A., Ritz, E., and Reichel, H. (1993). 1, 25-dihydroxyvitamin $\mathrm{d} 3$ production and vitamin $\mathrm{d} 3$ receptor expression are developmentally regulated during differentiation of human monocytes into macrophages. Cited on pages 15,71 , and 100 .

[129] Kriebitzsch, C., Verlinden, L., Eelen, G., Tan, B. K., Van Camp, M., Bouillon, R., and Verstuyf, A. (2009). The impact of 1, 25 (oh) $2 \mathrm{~d} 3$ and its structural analogs on gene expression in cancer cells-a microarray approach. Anticancer research, 29(9):3471-3483. Cited on page 97.

[130] Krishnan, A. V., Trump, D. L., Johnson, C. S., and Feldman, D. (2012). The role of vitamin $d$ in cancer prevention and treatment. Rheumatic Disease Clinics, 38(1):161-178. Cited on page 8 .

[131] Kundu, R., Theodoraki, A., Haas, C. T., Zhang, Y., Chain, B., Kriston-Vizi, J., Noursadeghi, M., and Khoo, B. (2017). Cell-type-specific modulation of innate immune signalling by vitamin d in human mononuclear phagocytes. Immunology, 150(1):55-63. Cited on page 101.

[132] Kutmon, M., Coort, S. L., de Nooijer, K., Lemmens, C., and Evelo, C. T. (2015a). Integrative network-based analysis of mrna and microrna expression in 1,25-dihydroxyvitamin d 3-treated cancer cells. Genes \& nutrition, 10(5):35. Cited on pages 8 and 97.

[133] Kutmon, M., Kelder, T., Mandaviya, P., Evelo, C. T., and Coort, S. L. (2013). Cytargetlinker: a cytoscape app to integrate regulatory interactions in network analysis. PloS one, 8(12):e82160. Cited on pages 24, 30, and 38.

[134] Kutmon, M., Lotia, S., Evelo, C. T., and Pico, A. R. (2014). Wikipathways app for cytoscape: Making biological pathways amenable to network analysis and visualization. F1000Research, 3. Cited on pages 24, 30, and 38.

[135] Kutmon, M., Riutta, A., Nunes, N., Hanspers, K., Willighagen, E. L., Bohler, A., Mélius, J., Waagmeester, A., Sinha, S. R., Miller, R., et al. (2015b). Wikipathways: capturing the full diversity of pathway knowledge. Nucleic acids research, 44(D1):D488D494. Cited on pages 52, 72, and 75.

[136] Kutmon, M., van Iersel, M. P., Bohler, A., Kelder, T., Nunes, N., Pico, A. R., and Evelo, C. T. (2015c). Pathvisio 3: an extendable pathway analysis toolbox. PLoS computational biology, 11(2):e1004085. Cited on pages 22, 23, 30, 37, 72, 75, and 122.

[137] Lake, J. E. and Adams, J. S. (2011). Vitamin d in hiv-infected patients. Current HIV/AIDS Reports, 8(3):133-141. Cited on page 70.

[138] Lampropoulou, V., Sergushichev, A., Bambouskova, M., Nair, S., Vincent, E. E., Loginicheva, E., Cervantes-Barragan, L., Ma, X., Huang, S. C.-C., Griss, T., et al. (2016). Itaconate links inhibition of succinate dehydrogenase with macrophage metabolic remodeling and regulation of inflammation. Cell metabolism, 24(1):158-166. Cited on page 117.

[139] Lash, G. E., Otun, H. A., Innes, B. A., Percival, K., Searle, R. F., Robson, S. C., and Bulmer, J. N. (2010). Regulation of extravillous trophoblast invasion by uterine natural killer cells is dependent on gestational age. Human reproduction, 25(5):1137-1145. Cited on page 49.

[140] Lash, G. E., Schiessl, B., Kirkley, M., Innes, B. A., Cooper, A., Searle, R. F., Robson, S. C., and Bulmer, J. N. (2006). Expression of angiogenic growth factors by uterine natural killer cells during early pregnancy. Journal of leukocyte biology, 80(3):572-580. 
Cited on page 49.

[141] Lightwood, R., Sheldon, W., Harris, C., and Stapleton, T. (1956). Hypercalcaemia in infants and vitamin d. BMJ, 2(4985):149-149. Cited on page 4.

[142] Lim, T. S., Goh, J. K. H., Mortellaro, A., Lim, C. T., Hämmerling, G. J., and RicciardiCastagnoli, P. (2012). Cd80 and cd86 differentially regulate mechanical interactions of t-cells with antigen-presenting dendritic cells and b-cells. PloS one, 7(9). Cited on page 11 .

[143] Lisse, T. S., Liu, T., Irmler, M., Beckers, J., Chen, H., Adams, J. S., and Hewison, M. (2011). Gene targeting by the vitamin d response element binding protein reveals a role for vitamin d in osteoblast mtor signaling. The FASEB Journal, 25(3):937-947. Cited on page 74.

[144] Litonjua, A. A., Carey, V. J., Laranjo, N., Stubbs, B. J., Mirzakhani, H., O’Connor, G. T., Sandel, M., Beigelman, A., Bacharier, L. B., Zeiger, R. S., et al. (2020). Six-year follow-up of a trial of antenatal vitamin d for asthma reduction. New England Journal of Medicine, 382(6):525-533. Cited on pages 126 and 127.

[145] Liu, P. T., Stenger, S., Li, H., Wenzel, L., Tan, B. H., Krutzik, S. R., Ochoa, M. T., Schauber, J., Wu, K., Meinken, C., et al. (2006). Toll-like receptor triggering of a vitamin d-mediated human antimicrobial response. Science, 311(5768):1770-1773. Cited on pages $16,71,95,98$, and 101 .

[146] Luan, H. H. and Medzhitov, R. (2016). Food fight: role of itaconate and other metabolites in antimicrobial defense. Cell metabolism, 24(3):379-387. Cited on page 117.

[147] Luckheeram, R. V., Zhou, R., Verma, A. D., and Xia, B. (2012). Cd4+ t cells: differentiation and functions. Clinical and developmental immunology, 2012. Cited on page 13.

[148] Lund, B. and Selnes, A. (1979). Plasma 1, 25-dihydroxyvitamin d levels in pregnancy and lactation. European Journal of Endocrinology, 92(2):330-335. Cited on page 4.

[149] Malinarich, F., Duan, K., Hamid, R. A., Bijin, A., Lin, W. X., Poidinger, M., Fairhurst, A.-M., and Connolly, J. E. (2015). High mitochondrial respiration and glycolytic capacity represent a metabolic phenotype of human tolerogenic dendritic cells. The Journal of Immunology, 194(11):5174-5186. Cited on page 116.

[150] Mangelsdorf, D. J., Koeffler, H. P., Donaldson, C. A., Pike, J. W., and Haussler, M. R. (1984). 1, 25-dihydroxyvitamin d3-induced differentiation in a human promyelocytic leukemia cell line (hl-60): receptor-mediated maturation to macrophage-like cells. The Journal of cell biology, 98(2):391-398. Cited on pages 15 and 71.

[151] Marin, E., Bouchet-Delbos, L., Renoult, O., Louvet, C., Nerriere-Daguin, V., Managh, A. J., Even, A., Giraud, M., Manh, T. P. V., Aguesse, A., et al. (2019). Human tolerogenic dendritic cells regulate immune responses through lactate synthesis. Cell Metabolism, 30(6):1075-1090. Cited on pages 116 and 119.

[152] Mascanfroni, I. D., Yeste, A., Vieira, S. M., Burns, E. J., Patel, B., Sloma, I., Wu, Y., Mayo, L., Ben-Hamo, R., Efroni, S., et al. (2013). Il-27 acts on des to suppress the t cell response and autoimmunity by inducing expression of the immunoregulatory molecule cd39. Nature immunology, 14(10):1054. Cited on page 12.

[153] Matyszak, M. K., Citterio, S., Rescigno, M., and Ricciardi-Castagnoli, P. (2000). Differential effects of corticosteroids during different stages of dendritic cell maturation. European journal of immunology, 30(4):1233-1242. Cited on page 12.

[154] McCollum, E. V. and Davis, M. (1913). The necessity of certain lipins in the diet during growth. Journal of Biological Chemistry, 15(1):167-175. Cited on page 2. 
[155] McCollum, E. V., Simmonds, N., Becker, J. E., and Shipley, P. (1922). Studies on experimental rickets xxi. an experimental demonstration of the existence of a vitamin which promotes calcium deposition. Journal of Biological Chemistry, 53(2):293-312. Cited on page 3.

[156] McCollum, E. V., Simmonds, N., and Pitz, W. (1916). The relation of the unidentified dietary factors, the fat-soluble $\mathrm{a}$, and watersoluble $\mathrm{b}$, of the diet to the growthpromoting properties of milk. Journal of Biological Chemistry, 27(1):33-43. Cited on page 2.

[157] Medrano, M., Carrillo-Cruz, E., Montero, I., and Perez-Simon, J. A. (2018). Vitamin $\mathrm{d}$ : effect on haematopoiesis and immune system and clinical applications. International journal of molecular sciences, 19(9):2663. Cited on page 128.

[158] Mellanby, E. (1976). Nutrition classics. the lancet 1: 407-12, 1919. an experimental investigation of rickets. edward mellanby. Nutrition reviews, 34(11):338. Cited on page 3 .

[159] Mellanby, S. E. (2013). The part played by an accessory factor in the production of experimental rickets. Cited on page 3 .

[160] Meyer, M. B., Benkusky, N. A., Lee, C.-H., and Pike, J. W. (2014). Genomic determinants of gene regulation by 1, 25-dihydroxyvitamin $\mathrm{d} 3$ during osteoblast-lineage cell differentiation. Journal of Biological Chemistry, 289(28):19539-19554. Cited on page 95 .

[161] Michalek, R. D., Gerriets, V. A., Jacobs, S. R., Macintyre, A. N., MacIver, N. J., Mason, E. F., Sullivan, S. A., Nichols, A. G., and Rathmell, J. C. (2011). Cutting edge: distinct glycolytic and lipid oxidative metabolic programs are essential for effector and regulatory cd4+ t cell subsets. The Journal of Immunology, 186(6):3299-3303. Cited on page 96.

[162] Michl, J., Ohlbaum, D. J., and Silverstein, S. C. (1976). 2-deoxyglucose selectively inhibits fc and complement receptor-mediated phagocytosis in mouse peritoneal macrophages. i. description of the inhibitory effect. The Journal of experimental medicine, 144(6):1465-1483. Cited on page 125.

[163] Mills, E. L., Ryan, D. G., Prag, H. A., Dikovskaya, D., Menon, D., Zaslona, Z., Jedrychowski, M. P., Costa, A. S., Higgins, M., Hams, E., et al. (2018). Itaconate is an anti-inflammatory metabolite that activates nrf2 via alkylation of keap1. Nature, 556(7699):113. Cited on page 117.

[164] Mlecnik, B., Galon, J., and Bindea, G. (2018). Comprehensive functional analysis of large lists of genes and proteins. Journal of proteomics, 171:2-10. Cited on page 24.

[165] Mócsai, A. (2013). Diverse novel functions of neutrophils in immunity, inflammation, and beyond. Journal of Experimental Medicine, 210(7):1283-1299. Cited on page 9.

[166] Moffett, A. and Colucci, F. (2014). Uterine nk cells: active regulators at the maternalfetal interface. The Journal of clinical investigation, 124(5):1872-1879. Cited on page 65.

[167] Moore, K. W., de Waal Malefyt, R., Coffman, R. L., and O'Garra, A. (2001). Interleukin-10 and the interleukin-10 receptor. Annual review of immunology, 19(1):683765. Cited on page 12.

[168] Mosieniak, G., Sliwinska, M., Piwocka, K., and Sikora, E. (2006). Curcumin abolishes apoptosis resistance of calcitriol-differentiated hl-60 cells. FEBS letters, 580(19):46534660. Cited on page 98.

[169] Mosser, D. M. and Edwards, J. P. (2008). Exploring the full spectrum of macrophage activation. Nature reviews immunology, 8(12):958-969. Cited on page 10.

[170] Nagai, T. (2017). Difference between immature dendritic cells (imdcs) and mature dendritic cells (mdcs) derived from human monocytes. Cited on page 12. 
[171] Nikolic, T. and Roep, B. (2013). Regulatory multitasking of tolerogenic dendritic cells-lessons taken from vitamin d3-treated tolerogenic dendritic cells. Frontiers in immunology, 4:113. Cited on page 101.

[172] Nnoaham, K. E. and Clarke, A. (2008). Low serum vitamin d levels and tuberculosis: a systematic review and meta-analysis. International journal of epidemiology, 37(1):113119. Cited on page 70.

[173] Nurminen, V., Neme, A., Ryynänen, J., Heikkinen, S., Seuter, S., and Carlberg, C. (2015). The transcriptional regulator bcl6 participates in the secondary gene regulatory response to vitamin d. Biochimica et Biophysica Acta (BBA)-Gene Regulatory Mechanisms, 1849(3):300-308. Cited on pages 74 and 94.

[174] Oakey, L. A., Fletcher, R. S., Elhassan, Y. S., Cartwright, D. M., Doig, C. L., Garten, A., Thakker, A., Maddocks, O. D., Zhang, T., Tennant, D. A., et al. (2018). Metabolic tracing reveals novel adaptations to skeletal muscle cell energy production pathways in response to nad+ depletion. Wellcome open research, 3. Cited on page 106.

[175] O'Neill, L. A., Kishton, R. J., and Rathmell, J. (2016). A guide to immunometabolism for immunologists. Nature Reviews Immunology, 16(9):553. Cited on page 115.

[176] O’Neill, L. A. and Pearce, E. J. (2016). Immunometabolism governs dendritic cell and macrophage function. Journal of Experimental Medicine, 213(1):15-23. Cited on page 116.

[177] Ota, K., Dambaeva, S., Han, A.-R., Beaman, K., Gilman-Sachs, A., and Kwak-Kim, J. (2014). Vitamin d deficiency may be a risk factor for recurrent pregnancy losses by increasing cellular immunity and autoimmunity. Human reproduction, 29(2):208-219. Cited on page 18.

[178] Ota, K., Dambaeva, S., Kim, M. W.-I., Han, A.-R., Fukui, A., Gilman-Sachs, A., Beaman, K., and Kwak-Kim, J. (2015). 1, 25-dihydroxy-vitamin d3 regulates nk-cell cytotoxicity, cytokine secretion, and degranulation in women with recurrent pregnancy losses. European journal of immunology, 45(11):3188-3199. Cited on page 18.

[179] Otasek, D., Morris, J. H., Bouças, J., Pico, A. R., and Demchak, B. (2019). Cytoscape automation: empowering workflow-based network analysis. Genome biology, 20(1):1-15. Cited on page 124.

[180] Owen, J. A., Punt, J., Stranford, S. A., et al. (2013). Kuby immunology. WH Freeman New York. Cited on page 12.

[181] Park, J. M., Park, C. Y., and Han, S. N. (2015). High fat diet-induced obesity alters vitamin d metabolizing enzyme expression in mice. Biofactors, 41(3):175-182. Cited on page 8 .

[182] Parkinson, H., Kapushesky, M., Shojatalab, M., Abeygunawardena, N., Coulson, R., Farne, A., Holloway, E., Kolesnykov, N., Lilja, P., Lukk, M., et al. (2006). Arrayexpress-a public database of microarray experiments and gene expression profiles. Nucleic acids research, 35(suppl_1):D747-D750. Cited on page 29.

[183] Patil, K. R. and Nielsen, J. (2005). Uncovering transcriptional regulation of metabolism by using metabolic network topology. Proceedings of the national academy of sciences, 102(8):2685-2689. Cited on page 38.

[184] Paul, S. and Lal, G. (2017). The molecular mechanism of natural killer cells function and its importance in cancer immunotherapy. Frontiers in immunology, 8:1124. Cited on page 17.

[185] Pavlou, S., Wang, L., Xu, H., and Chen, M. (2017). Higher phagocytic activity of thioglycollate-elicited peritoneal macrophages is related to metabolic status of the cells. Journal of inflammation, 14(1):4. Cited on page 125. 
[186] Penna, G. and Adorini, L. (2000). 1 $\alpha$, 25-dihydroxyvitamin d3 inhibits differentiation, maturation, activation, and survival of dendritic cells leading to impaired alloreactive t cell activation. The Journal of Immunology, 164(5):2405-2411. Cited on pages 17 and 101.

[187] Peshes-Yaloz, N., Rosen, D., Sondel, P. M., Krammer, P. H., and Berke, G. (2007). Upregulation of fas (cd95) expression in tumour cells in vivo. Immunology, 120(4):502-511. Cited on page 15.

[188] Pesta, D. and Gnaiger, E. (2012). High-resolution respirometry: Oxphos protocols for human cells and permeabilized fibers from small biopsies of human muscle. In Mitochondrial bioenergetics, pages 25-58. Springer. Cited on page 105.

[189] Picarda, E., Ohaegbulam, K. C., and Zang, X. (2016). Molecular pathways: targeting b7-h3 (cd276) for human cancer immunotherapy. Clinical Cancer Research, 22(14):34253431. Cited on page 53.

[190] Piemonti, L., Monti, P., Sironi, M., Fraticelli, P., Leone, B. E., Dal Cin, E., Allavena, P., and Di Carlo, V. (2000). Vitamin d3 affects differentiation, maturation, and function of human monocyte-derived dendritic cells. The Journal of Immunology, 164(9):4443-4451. Cited on page 101.

[191] Pike, J. W., Meyer, M. B., Lee, S.-M., Onal, M., and Benkusky, N. A. (2017). The vitamin d receptor: contemporary genomic approaches reveal new basic and translational insights. The Journal of clinical investigation, 127(4):1146-1154. Cited on page 95.

[192] Pillich, R. T., Chen, J., Rynkov, V., Welker, D., and Pratt, D. (2017). Ndex: a community resource for sharing and publishing of biological networks. In Protein Bioinformatics, pages 271-301. Springer. Cited on page 44.

[193] Pilz, S., März, W., Cashman, K. D., Kiely, M. E., Whiting, S. J., Holick, M. F., Grant, W. B., Pludowski, P., Hiligsmann, M., Trummer, C., et al. (2018). Rationale and plan for vitamin d food fortification: a review and guidance paper. Frontiers in endocrinology, 9:373. Cited on page 4.

[194] Pittas, A. G., Dawson-Hughes, B., Sheehan, P., Ware, J. H., Knowler, W. C., Aroda, V. R., Brodsky, I., Ceglia, L., Chadha, C., Chatterjee, R., et al. (2019). Vitamin d supplementation and prevention of type 2 diabetes. Cited on pages 126 and 127.

[195] Plitas, G. and Rudensky, A. Y. (2016). Regulatory t cells: differentiation and function. Cancer immunology research, 4(9):721-725. Cited on page 14.

[196] Poli, A., Michel, T., Thérésine, M., Andrès, E., Hentges, F., and Zimmer, J. (2009). Cd56bright natural killer (nk) cells: an important nk cell subset. Immunology, 126(4):458465. Cited on pages 53 and 65.

[197] Pollheimer, J., Vondra, S., Baltayeva, J., Beristain, A. G., and Knöfler, M. (2018). Regulation of placental extravillous trophoblasts by the maternal uterine environment. Frontiers in immunology, 9:2597. Cited on page 66.

[198] Provvedini, D. M., Tsoukas, C. D., Deftos, L. J., and Manolagas, S. C. (1983). 1, 25-dihydroxyvitamin d3 receptors in human leukocytes. Science, 221(4616):1181-1183. Cited on pages 15 and 17.

[199] Rae, C. and Graham, A. (2008). Fatty acid synthase inhibitor, c75, blocks resistininduced increases in lipid accumulation by human macrophages. Diabetes, Obesity and Metabolism, 10(12):1271-1274. Cited on page 115.

[200] Raker, V. K., Domogalla, M. P., and Steinbrink, K. (2015). Tolerogenic dendritic cells for regulatory t cell induction in man. Frontiers in immunology, 6:569. Cited on page 12.

[201] Rehman, A., Hemmert, K. C., Ochi, A., Jamal, M., Henning, J. R., Barilla, R., Quesada, J. P., Zambirinis, C. P., Tang, K., Ego-Osuala, M., et al. (2013). Role of fatty-acid synthesis in dendritic cell generation and function. The Journal of Immunology, 190(9):4640-4649. 
Cited on page 118 .

[202] Rendina, A. R. and Cheng, D. (2005). Characterization of the inactivation of rat fatty acid synthase by c75: inhibition of partial reactions and protection by substrates. Biochemical Journal, 388(3):895-903. Cited on page 115.

[203] Rigby, W., Noelle, R. J., Krause, K., and Fanger, M. (1985). The effects of 1, $25-$ dihydroxyvitamin $\mathrm{d} 3$ on human $\mathrm{t}$ lymphocyte activation and proliferation: a cell cycle analysis. The Journal of immunology, 135(4):2279-2286. Cited on page 17.

[204] Rincón, M. and Flavell, R. A. (1997). Transcriptional control in the th1/th2 decision: T-cell subsets. Current Biology, 7(11):R729-R732. Cited on page 12.

[205] Ritchie, M. E., Carvalho, B. S., Hetrick, K. N., Tavaré, S., and Irizarry, R. A. (2009). $\mathrm{R} /$ bioconductor software for illumina's infinium whole-genome genotyping beadchips. Bioinformatics, 25(19):2621-2623. Cited on page 74.

[206] Ritchie, M. E., Phipson, B., Wu, D., Hu, Y., Law, C. W., Shi, W., and Smyth, G. K. (2015). limma powers differential expression analyses for rna-sequencing and microarray studies. Nucleic acids research, 43(7):e47-e47. Cited on page 34.

[207] Roberts, L. (2001). Timeline: A history of the human genome project. Science, 291(5507):1195-1200. Cited on page 95.

[208] Robinson, C. J., Wagner, C. L., Hollis, B. W., Baatz, J. E., and Johnson, D. D. (2011). Maternal vitamin $\mathrm{d}$ and fetal growth in early-onset severe preeclampsia. American journal of obstetrics and gynecology, 204(6):556-e1. Cited on page 65.

[209] Robson, A., Harris, L. K., Innes, B. A., Lash, G. E., Aljunaidy, M. M., Aplin, J. D., Baker, P. N., Robson, S. C., and Bulmer, J. N. (2012). Uterine natural killer cells initiate spiral artery remodeling in human pregnancy. The FASEB Journal, 26(12):4876-4885. Cited on page 15.

[210] Romagnani, S. (1999). Th1/th2 cells. Inflammatory bowel diseases, 5(4):285-294. Cited on page 12.

[211] Ross, A. C., Taylor, C. L., Yaktine, A. L., and Del Valle, H. B. (2011). Committee to review dietary reference intakes for vitamin $\mathrm{d}$ and calcium. Food and Nutrition Board. Cited on page 127.

[212] Rőszer, T. (2015). Understanding the mysterious $\mathrm{m} 2$ macrophage through activation markers and effector mechanisms. Mediators of inflammation, 2015. Cited on page 12.

[213] Saul, L., Mair, I., Ivens, A., Brown, P., Samuel, K., Campbell, J. D., Soong, D. Y., Kamenjarin, N., and Mellanby, R. J. (2019). 1, 25-dihydroxyvitamin d3 restrains cd4+ t cell priming ability of cd11c+ dendritic cells by upregulating expression of cd31. Frontiers in immunology, 10:600. Cited on page 101.

[214] Seida, J. C., Mitri, J., Colmers, I. N., Majumdar, S. R., Davidson, M. B., Edwards, A. L., Hanley, D. A., Pittas, A. G., Tjosvold, L., and Johnson, J. A. (2014). Effect of vitamin $\mathrm{d} 3$ supplementation on improving glucose homeostasis and preventing diabetes: a systematic review and meta-analysis. The Journal of Clinical Endocrinology \& Metabolism, 99(10):3551-3560. Cited on page 97.

[215] Seoane, S. and Perez-Fernandez, R. (2006). The vitamin d receptor represses transcription of the pituitary transcription factor pit- 1 gene without involvement of the retinoid x receptor. Molecular endocrinology, 20(4):735-748. Cited on page 7.

[216] Seuter, S., Neme, A., and Carlberg, C. (2015). Epigenome-wide effects of vitamin d and their impact on the transcriptome of human monocytes involve ctcf. Nucleic acids research, 44(9):4090-4104. Cited on pages 94 and 97.

[217] Seuter, S., Neme, A., and Carlberg, C. (2016). Epigenome-wide effects of vitamin d and their impact on the transcriptome of human monocytes involve ctcf. Nucleic acids 
research, 44(9):4090-4104. Cited on page 67.

[218] Seyedalipour, F., Mansouri, A., Vaezi, M., Gholami, K., Heidari, K., Hadjibabaie, M., and Ghavamzadeh, A. (2017). High prevalence of vitamin d deficiency in newly diagnosed acute myeloid leukemia patients and its adverse outcome. International journal of hematology-oncology and stem cell research, 11(3):209. Cited on page 127.

[219] Shannon, P., Markiel, A., Ozier, O., Baliga, N. S., Wang, J. T., Ramage, D., Amin, N., Schwikowski, B., and Ideker, T. (2003). Cytoscape: a software environment for integrated models of biomolecular interaction networks. Genome research, 13(11):2498-2504. Cited on page 23 .

[220] Singh, P. K., van den Berg, P. R., Long, M. D., Vreugdenhil, A., Grieshober, L., Ochs-Balcom, H. M., Wang, J., Delcambre, S., Heikkinen, S., Carlberg, C., et al. (2017). Integration of vdr genome wide binding and gwas genetic variation data reveals cooccurrence of vdr and $\mathrm{nf}-\kappa \mathrm{b}$ binding that is linked to immune phenotypes. BMC genomics, 18(1):132. Cited on page 95.

[221] Slenter, D. N., Kutmon, M., Hanspers, K., Riutta, A., Windsor, J., Nunes, N., Mélius, J., Cirillo, E., Coort, S. L., Digles, D., et al. (2017). Wikipathways: a multifaceted pathway database bridging metabolomics to other omics research. Nucleic acids research, 46(D1):D661-D667. Cited on pages 30, 35, and 122.

[222] Slenter, D. N., Kutmon, M., Hanspers, K., Riutta, A., Windsor, J., Nunes, N., Mélius, J., Cirillo, E., Coort, S. L., Digles, D., et al. (2018). Wikipathways: a multifaceted pathway database bridging metabolomics to other omics research. Nucleic acids research, 46(D1):D661-D667. Cited on pages 21 and 22.

[223] Smoot, M. E., Ono, K., Ruscheinski, J., Wang, P.-L., and Ideker, T. (2010). Cytoscape 2.8: new features for data integration and network visualization. Bioinformatics, 27(3):431432. Cited on pages 30, 38, 72, 77, and 124 .

[224] Sojka, D. K., Yang, L., and Yokoyama, W. M. (2019). Uterine natural killer cells. Frontiers in immunology, 10:960. Cited on page 18.

[225] Song, G. G., Bae, S.-C., and Lee, Y. H. (2012). Association between vitamin d intake and the risk of rheumatoid arthritis: a meta-analysis. Clinical rheumatology, 31(12):1733-1739. Cited on page 127.

[226] Steenbock, H. (1924). The induction of growth promoting and calcifying properties in a ration by exposure to light. Science, 60(1549):224-225. Cited on page 3.

[227] Steinman, R. (2003). The control of immunity and tolerance by dendritic cells. Pathologie Biologie, 51(2):59-60. Cited on page 12.

[228] Steinman, R. M. and Nussenzweig, M. C. (2002). Avoiding horror autotoxicus: the importance of dendritic cells in peripheral t cell tolerance. Proceedings of the National Academy of Sciences, 99(1):351-358. Cited on page 11.

[229] Stephen, J. M. (1975). Epidemiological and dietary aspects of rickets and osteomalacia. Proceedings of the Nutrition Society, 34(2):131-138. Cited on page 4.

[230] Szekeres-Bartho, J. (2008). Regulation of nk cell cytotoxicity during pregnancy. Reproductive biomedicine online, 16(2):211-217. Cited on page 49.

[231] Széles, L., Keresztes, G., Töröcsik, D., Balajthy, Z., Krenács, L., Póliska, S., Steinmeyer, A., Zuegel, U., Pruenster, M., Rot, A., et al. (2009). 1, 25-dihydroxyvitamin d3 is an autonomous regulator of the transcriptional changes leading to a tolerogenic dendritic cell phenotype. The Journal of Immunology, 182(4):2074-2083. Cited on pages 29, 34, $36,41,43$, and 74 .

[232] Taglauer, E. S., Waldorf, K. M. A., and Petroff, M. G. (2010). The hidden maternalfetal interface: events involving the lymphoid organs in maternal-fetal tolerance. The 
International journal of developmental biology, 54(2-3):421. Cited on page 48.

[233] Takahashi, K., Nakayama, Y., Horiuchi, H., Ohta, T., Komoriya, K., Ohmori, H., and Kamimura, T. (2002). Human neutrophils express messenger rna of vitamin d receptor and respond to $1 \alpha, 25$-dihydroxyvitamin $\mathrm{d} 3$. Immunopharmacology and immunotoxicology, 24(3):335-347. Cited on page 15.

[234] Talaei, A., Mohamadi, M., and Adgi, Z. (2013). The effect of vitamin d on insulin resistance in patients with type 2 diabetes. Diabetology \& metabolic syndrome, 5(1):8. Cited on page 127.

[235] Tamblyn, J., Susarla, R., Jenkinson, C., Jeffery, L., Ohizua, O., Chun, R., Chan, S. Y., Kilby, M., and Hewison, M. (2017). Dysregulation of maternal and placental vitamin d metabolism in preeclampsia. Placenta, 50:70-77. Cited on pages 49 and 68.

[236] Tamblyn, J. A., Jeffery, L., Susarla, R., Lissauer, D. M., Coort, S. L., García, A. M., Knoblich, K., Fletcher, A. L., Bulmer, J. N. N., Kilby, M., et al. (2019). Transcriptomic analysis of vitamin d responses in uterine and peripheral nk cells. Reproduction, 1(aop). Cited on pages 130 and 136.

[237] Théry, C. and Amigorena, S. (2001). The cell biology of antigen presentation in dendritic cells. Current opinion in immunology, 13(1):45-51. Cited on page 11.

[238] Vacca, P., Moretta, L., Moretta, A., and Mingari, M. C. (2011). Origin, phenotype and function of human natural killer cells in pregnancy. Trends in immunology, 32(11):517523. Cited on page 67.

[239] Vacca, P., Pietra, G., Falco, M., Romeo, E., Bottino, C., Bellora, F., Prefumo, F., Fulcheri, E., Venturini, P. L., Costa, M., et al. (2006). Analysis of natural killer cells isolated from human decidua: evidence that $2 \mathrm{~b} 4(\mathrm{~cd} 244)$ functions as an inhibitory receptor and blocks nk-cell function. Blood, 108(13):4078-4085. Cited on page 66.

[240] van Halteren, A. G., van Etten, E., de Jong, E. C., Bouillon, R., Roep, B. O., and Mathieu, C. (2002). Redirection of human autoreactive t-cells upon interaction with dendritic cells modulated by tx 527, an analog of 1, 25 dihydroxyvitamin d3. Diabetes, 51(7):2119-2125. Cited on page 101.

[241] van Iersel, M. P., Kelder, T., Pico, A. R., Hanspers, K., Coort, S., Conklin, B. R., and Evelo, C. (2008). Presenting and exploring biological pathways with pathvisio. BMC bioinformatics, 9(1):399. Cited on pages 72 and 75.

[242] van Iersel, M. P., Pico, A. R., Kelder, T., Gao, J., Ho, I., Hanspers, K., Conklin, B. R., and Evelo, C. T. (2010). The bridgedb framework: standardized access to gene, protein and metabolite identifier mapping services. BMC bioinformatics, 11(1):5. Cited on pages 22,35 , and 75 .

[243] Vanherwegen, A.-S., Eelen, G., Ferreira, G. B., Ghesquière, B., Cook, D. P., Nikolic, T., Roep, B., Carmeliet, P., Telang, S., Mathieu, C., et al. (2019). Vitamin d controls the capacity of human dendritic cells to induce functional regulatory $\mathrm{t}$ cells by regulation of glucose metabolism. The Journal of steroid biochemistry and molecular biology, 187:134-145. Cited on pages 117 and 126.

[244] Vanherwegen, A.-S., Gysemans, C., and Mathieu, C. (2017). Vitamin d endocrinology on the cross-road between immunity and metabolism. Molecular and cellular endocrinology, 453:52-67. Cited on page 95.

[245] Varol, C., Mildner, A., and Jung, S. (2015). Macrophages: development and tissue specialization. Annual review of immunology, 33:643-675. Cited on page 10.

[246] Vats, D., Mukundan, L., Odegaard, J. I., Zhang, L., Smith, K. L., Morel, C. R., Greaves, D. R., Murray, P. J., and Chawla, A. (2006). Oxidative metabolism and pgc- $1 \beta$ attenuate macrophage-mediated inflammation. Cell metabolism, 4(1):13-24. Cited on page 96. 
[247] Vento-Tormo, R., Efremova, M., Botting, R. A., Turco, M. Y., Vento-Tormo, M., Meyer, K. B., Park, J.-E., Stephenson, E., Polański, K., Goncalves, A., et al. (2018). Singlecell reconstruction of the early maternal-fetal interface in humans. Nature, 563(7731):347353. Cited on pages 67 and 68.

[248] Verway, M., Bouttier, M., Wang, T.-T., Carrier, M., Calderon, M., An, B.-S., Devemy, E., McIntosh, F., Divangahi, M., Behr, M. A., et al. (2013). Vitamin d induces interleukin- $1 \beta$ expression: paracrine macrophage epithelial signaling controls $\mathrm{m}$. tuberculosis infection. PLoS pathogens, 9(6):e1003407. Cited on pages 34, 36, 41, 43, and 74.

[249] Vivier, E., Raulet, D. H., Moretta, A., Caligiuri, M. A., Zitvogel, L., Lanier, L. L., Yokoyama, W. M., and Ugolini, S. (2011). Innate or adaptive immunity? the example of natural killer cells. Science, 331(6013):44-49. Cited on page 66.

[250] Wagner, C. L., Taylor, S. N., Johnson, D. D., and Hollis, B. W. (2012). The role of vitamin d in pregnancy and lactation: emerging concepts. Women's Health, 8(3):323-340. Cited on page 4.

[251] Walsh, J. S., Bowles, S., and Evans, A. L. (2017). Vitamin d in obesity. Current Opinion in Endocrinology \& Diabetes and Obesity, 24(6):389-394. Cited on page 8.

[252] Wang, F., Zhou, Y., Fu, B., Wu, Y., Zhang, R., Sun, R., Tian, Z., and Wei, H. (2014). Molecular signatures and transcriptional regulatory networks of human immature decidual nk and mature peripheral nk cells. European journal of immunology, 44(9):2771-2784. Cited on page 66.

[253] Wang, H., Marcišauskas, S., Sánchez, B. J., Domenzain, I., Hermansson, D., Agren, R., Nielsen, J., and Kerkhoven, E. J. (2018). Raven 2.0: A versatile toolbox for metabolic network reconstruction and a case study on streptomyces coelicolor. PLoS computational biology, 14(10):e1006541. Cited on page 24.

[254] Wang, T.-T., Nestel, F. P., Bourdeau, V., Nagai, Y., Wang, Q., Liao, J., Tavera-Mendoza, L., Lin, R., Hanrahan, J. W., Mader, S., et al. (2004). Cutting edge: 1, 25-dihydroxyvitamin $\mathrm{d} 3$ is a direct inducer of antimicrobial peptide gene expression. The Journal of Immunology, 173(5):2909-2912. Cited on page 16.

[255] Wei, R. and Christakos, S. (2015). Mechanisms underlying the regulation of innate and adaptive immunity by vitamin d. Nutrients, 7(10):8251-8260. Cited on page 70 .

[256] Weiskopf, K., Schnorr, P. J., Pang, W. W., Chao, M. P., Chhabra, A., Seita, J., Feng, M., and Weissman, I. L. (2017). Myeloid cell origins, differentiation, and clinical implications. Myeloid Cells in Health and Disease: A Synthesis, pages 857-875. Cited on page 9.

[257] Weisman, Y., Harell, A., Edelstein, S., David, M., Spirer, Z., and Golander, A. 1. (1979). $1 \alpha, 25$-dihydroxyvitamin $\mathrm{d} 3$, and 24, 25-dihydroxyvitamin $\mathrm{d} 3$ in vitro synthesis by human decidua and placenta. Nature, 281(5729):317-319. Cited on page 49.

[258] Wheelwright, M., Kim, E. W., Inkeles, M. S., De Leon, A., Pellegrini, M., Krutzik, S. R., and Liu, P. T. (2014). All-trans retinoic acid-triggered antimicrobial activity against mycobacterium tuberculosis is dependent on npc2. The Journal of Immunology, 192(5):2280-2290. Cited on page 74.

[259] Wilkinson, M. D., Dumontier, M., Aalbersberg, I. J., Appleton, G., Axton, M., Baak, A., Blomberg, N., Boiten, J.-W., da Silva Santos, L. B., Bourne, P. E., et al. (2016). The fair guiding principles for scientific data management and stewardship. Scientific data, 3. Cited on pages 20 and 121.

[260] Wing, K., Onishi, Y., Prieto-Martin, P., Yamaguchi, T., Miyara, M., Fehervari, Z., Nomura, T., and Sakaguchi, S. (2008). Ctla-4 control over foxp3+ regulatory t cell function. Science, 322(5899):271-275. Cited on page 14. 
[261] Wishart, D. S., Feunang, Y. D., Guo, A. C., Lo, E. J., Marcu, A., Grant, J. R., Sajed, T., Johnson, D., Li, C., Sayeeda, Z., et al. (2017). Drugbank 5.0: a major update to the drugbank database for 2018. Nucleic acids research, 46(D1):D1074-D1082. Cited on page 38 .

[262] Wu, D., Woods, P. S., Duong, H. T., Mutlu, G. M., Kim, B., Li, J., Jang, C., Arany, Z., De Bock, K., Georgiadou, M., et al. (2018). Tlr-driven early glycolytic reprogramming via the kinases tbk1-ikkvarepsilon supports the anabolic demands of dendritic cell activation. American Journal of Respiratory Cell and Molecular Biology, 59(1):127-129. Cited on page 116.

[263] Yuk, J.-M., Shin, D.-M., Lee, H.-M., Yang, C.-S., Jin, H. S., Kim, K.-K., Lee, Z.-W., Lee, S.-H., Kim, J.-M., and Jo, E.-K. (2009). Vitamin d3 induces autophagy in human monocytes/macrophages via cathelicidin. Cell host \& microbe, 6(3):231-243. Cited on page 101 .

[264] Zehnder, D., Bland, R., Williams, M. C., McNinch, R. W., Howie, A. J., Stewart, P. M., and Hewison, M. (2001). Extrarenal expression of 25-hydroxyvitamin d3-1 $\alpha$-hydroxylase. The Journal of Clinical Endocrinology \& Metabolism, 86(2):888-894. Cited on page 16.

[265] Zehnder, D., Evans, K. N., Kilby, M. D., Bulmer, J. N., Innes, B. A., Stewart, P. M., and Hewison, M. (2002). The ontogeny of 25-hydroxyvitamin d3 $1 \alpha$-hydroxylase expression in human placenta and decidua. The American journal of pathology, 161(1):105-114. Cited on page 49.

[266] Zeyda, M., Säemann, M. D., Stuhlmeier, K. M., Mascher, D. G., Nowotny, P. N., Zlabinger, G. J., Waldhäusl, W., and Stulnig, T. M. (2005). Polyunsaturated fatty acids block dendritic cell activation and function independently of $\mathrm{nf}-\kappa \mathrm{b}$ activation. Journal of Biological Chemistry, 280(14):14293-14301. Cited on page 118.

[267] Zhang, N. and Bevan, M. J. (2011). Cd8+t cells: foot soldiers of the immune system. Immunity, 35(2):161-168. Cited on page 13.

[268] Zourbas, S., Dubanchet, S., Martal, J., and Chaouat, G. (2001). Localization of pro-inflammatory (il-12, il-15) and anti-inflammatory (il-11, il-13) cytokines at the foetomaternal interface during murine pregnancy. Clinical \& Experimental Immunology, 126(3):519-528. Cited on page 65.

[269] Zuo, R.-J., Gu, X.-W., Qi, Q.-R., Wang, T.-S., Zhao, X.-Y., Liu, J.-L., and Yang, Z.-M. (2015). Warburg-like glycolysis and lactate shuttle in mouse decidua during early pregnancy. Journal of Biological Chemistry, 290(35):21280-21291. Cited on page 66. 



\section{Appendix A}

\section{List of Abbreviations}

- 1,25(OH) ${ }_{2} \mathrm{D} 3$ : 1,25-dihydroxyvitamin D

- 25(OH)D: 25-hydroxyvitamin D

- ACO2: aconitase 2

- AE: Array Express

- AE: average expression

- AMP: adenosine monophosphate

- ANOVA: analysis of variance

- APAF: apoptotic peptidase-activating factor

- APC: antigen-presenting cells

- ATP: adenosine triphosphate

- BCR: B cell receptor

- BUB: budding uninhibited by benzimidazoles

- C75: 4-Methylene-2-octyl-5-oxotetrahydrofuran-3-carboxylic acid

- CD: cluster designation

- CDK: cyclin-dependent kinase

- CEBPB: CCAAT/enhancer-binding protein beta 
- CK: cytokine

- CLP: common lymphoid progenitor

- CYCS: cytochrome C, Somatic

- CYP24A1 : vitamin D3 24-hydroxylase

- CYP27B1: 25-hydroxyvitamin D-1 $\alpha$-hydroxylase

- CYP: P450 enzymes

- CaMKII: $\mathrm{Ca}^{2+} /$ calmodulin-dependent kinase II

- ChIP-Seq: immunoprecipitation-sequencing

- DAPI: 4',6-Diamidino-2-Phenylindole

- DAPK1: death-associated protein kinase 1

- DC: dendritic cell

- DEFB4A: $\beta$-defensin 4A

- DEX: dexamethasone

- DLD: dihydrolipoamide dehydrogenase

- DMEM: Dulbecco's modified eagle medium

- DNA: deoxyribonucleic acid

- E. coli: Escherichia coli

- ELISA: enzyme-linked immunosorbent assay

- ERK: extracellular-signal-regulated kinase

- ETC: electron transport chain

- FACS: fluorescence-activated cell sorting

- FAIR: findable, accessible, interoperable and reusable

- FAO: fatty acid oxidation

- FAS: fatty acid synthase 
- FBP: fructose-bisphosphatase

- FBS: foetal bovine serum

- FC: fold change

- FCCP: carbonyl cyanide-4-(trifluoromethoxy)phenylhydrazone

- FCS: fetal calf serum

- FDR: false discovery rate

- FNIP: folliculin-interacting protein

- FXN: frataxin

- FXN: frataxin

- GA: gestational age

- GEO: Gene Expression Omnibus

- GM-CSF: granulocyte macrophage-stimulating colony factor

- GMNN: Geminin

- GO: gene ontology

- GWAS: genome-wide association study

- GZMK: granzyme

- HGNC: HUGO Gene Nomenclature Committee

- HIF: hypoxia-inducible factor

- HK: Hexokinase

- HLA : human leukocyte antigens

- ID: identifier

- IDH: isocitrate dehydrogenase

- IL: interleukin

- INF: interferon 
- IOM: institute of medicine

- IR: infrared

- ITGAV: integrin alpha V

- Ig: immunoglobulin

- JAK: janus kinase

- JNK: c-Jun N-terminal kinas

- LPS: lipopolysaccharide

- M. tb: Mycobacterium tuberculosis

- MACS: magnetic-activated cell sorting

- MAPK: mitogen-activated protein kinase

- MDH2: malate dehydrogenase

- MFI: median fluorescence intensity

- MHC: major histocompatibility complex

- MTIs: microRNA-target interactions

- NAD: nicotinamide adenine dinucleotide

- NCBI: national center for biotechnology information

- NDEx: the networks data exchange

- NDUFS: NADH dehydrogenase [ubiquinone] iron-sulfur protein

- NHGRI: human genome research institute

- NK: natural killer

- $\mathrm{NaCl}$ : sodium chloride

- OCR: oxygen consumption rates

- ORC: origin recognition complex

- OXPHOS: oxidative phosphorylation 
- PAMPs: pathogen-associated molecular patterns

- PBMCs: peripheral blood mononuclear cells

- PBS: phosphate buffered saline

- PCA: principal component analysis

- PFA: paraformaldehyde

- PFKM: phosphofructokinase

- PI3K: phosphoinositide 3-kinase

- PKC: protein kinase $\mathrm{C}$

- PLAA: phospholipase $\mathrm{A}_{2}$ activating protein

- PPAR: peroxisomal proliferator-activated receptor

- PRIM: primase

- PRR: pattern-recognition receptors

- PTH: parathyroid hormone

- Provitamin D3: 7-dehydrocholesterol

- QC: quality control

- RAVEN : Reconstruction, Analysis and Visualization of Metabolic Networks

- RFC: replication factor $\mathrm{C}$

- RMA: robust multi-array average

- RNA: ribonucleic acid

- ROS: reactive oxygen species

- RPL: recurrent pregnancy loss

- RPMI: Roswell park memorial institute

- RT-PCR: reverse transcription polymerase chain reaction

- RXR: Retinoid X receptor 
- RXRA: Retinoid X receptor alpha

- SACN: Science Advisory Council on Nutrition

- SDH: succinate dehydrogenase

- SNP: single-nucleotide polymorphism

- SUCLG: succinyl-CoA ligase

- TCA: tricarboxylic acid

- TCR: T cell receptors

- TGF $\beta$ : transforming growth factor $\beta$

- TNF: tumour necrosis factor

- TNFSF: tumor necrosis factor (ligand) superfamily

- TOP: termination of pregnancy

- TRAP: TNF Receptor Associated Protein

- Tc: T cytotoxic

- Tfh: follicular T cells

- Th: T helper

- Tn: naïve T cell

- Treg: T regulatory cells

- UVB: ultra-violet B

- VDR: Vitamin D receptor

- VDRE: vitamin D response elements

- Vitamin D binding protein: DBP

- Vitamin D2: calciferol

- Vitamin D3: cholecalciferol

- WP: WikiPathways 
- YBX: Y-Box Binding Protein

- iDC: immature dendritic cell

- mDC: mature dendritic cell

- mRNA: messenger ribonucleic acid

- miRNA: microRNAs

- pNK: peripheral natural killer cells

- tolDC: tolerogenic dendritic cell

- uNK: uterine natural killer cells 



\section{Appendix B}

\section{Curriculum Vitae}

\section{$\underline{\text { Education }}$}

- Graduated in Biochemistry University of Valencia

$2009-2013$

- Master in Biotechology Polytechnic University of Valencia

$2013-2015$

- Master in Bioinformatics University of Murcia

$2015-2016$

- PhD Maastricht University \& University of Birmingham

$2016-2020$

- Postdoc Leiden University

2020 - Present

\section{$\underline{\text { Publications }}$}

- Pathway analysis of transcriptomic data shows immunometabolic effects of vitamin D Journal of Molecular Endocrinology

- A bioinformatics workflow to decipher transcriptomic data from vitamin D studies The Journal of Steroid Biochemistry and Molecular Biology

- Transcriptomic analysis of vitamin D responses in uterine and peripheral NK cells Reproduction

- Over-expression of the vitamin D receptor (VDR) induces skeletal muscle hypertrophy Molecular Metabolism

- The mechanisms of skeletal muscle atrophy in response to transient knockdown of the vitamin D receptor (VDR) in vivo Submitted to Molecular Metabolism 2020

- Tolerogenic effects of vitamin D on dendritic cells involve induction of fatty acid synthesis Submitted to Journal of Endocrinology 
Talks, posters, teaching and awards

- BioSB - Talk De Werelt - The Netherlands 2017

- Bioinformatics MSc student supervision Maastricht - The Netherlands 2017

- Vitamin D workshop - Talk \& Trainee Travel Award Barcelona - Spain 2018

- NuGO - Poster Newcastle - UK 2018

- Mechanisms of Inflammation Symposium - Talk Brimingham - UK 2018

- Laboratory MSc student supervision Brimingham - UK $2018-2019$

- Pathway analysis workshops Brimingham - UK 2018 - 2019

- 1st NUTRIM Division Symposium - Talk Maastricht - The Netherlands 2019 

